\title{
Quantum Confinement of Chiral Charge Carriers in Ring Conductors
}

\author{
Lei Yang
}

\section{VICTORIA UNIVERSITY OF WELLINGTON}

Te Whare Wānanga o te Ūpoko o te Ika a Māui

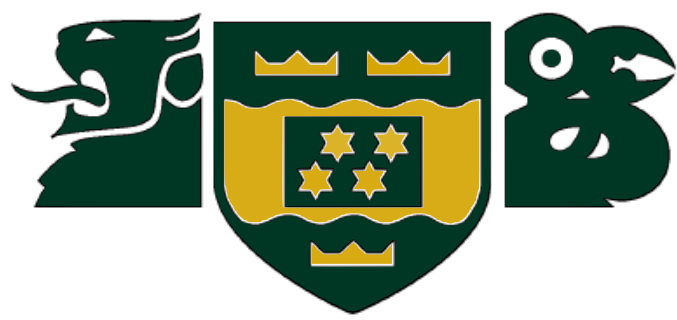

School of Chemical and Physical Sciences Te Wānanga Matū

A thesis

submitted to the Victoria University of Wellington in fulfilment of the requirements for the degree of Master of Science in Physics.

Victoria University of Wellington 



\begin{abstract}
We theoretically study the quantum confinement effects and transport properties of quantum ring (QR) systems. In particular, we investigate QRs made out of the following materials: single-layer graphene (SLG), singlelayer transition-metal dichalcogenides (TMDs) and narrow-gap semiconductor quantum wells (SQWs).

Via perturbation theory and assuming that the ring aspect ratio is small, the general subband dispersion relations of these hard-wall ring confined systems are determined. These dispersion results agree with and extend on previous works. We discover the necessity of including both a size-quantisation energy and an angular momentum dependent energy shift to the dispersion equation due to their sizeable impact on the conductance of the system.

The topological properties of these QR systems is also investigated. We find that QR confinement of materials may destroy the topologically non-trivial properties of states. The topological phase can be recovered when the band structure is inverted and the confined material parameters satisfy certain critical widths and gap limits.

An analytical expression of the conductance for QRs (with symmetricallyarranged leads), in the presence of the perpendicular magnetic field piercing the centre of the ring, is derived. We study the geometric (i.e. Berry) and dynamic phases of the system that arise from the interference of partial waves in the ring branches. We discover that the Berry phase is modified by a correction term that arises purely from the quantum confinement of the materials. This has generally not been taken into account by previous studies. The explicit analytical expressions of the phase correction term are derived and shown to be proportional to the angular momentum dependent energy shift, present in the dispersion relations, for lead injection energies close to the subband energy.

Overall, this study finds that the material-dependent phase plays a significant role in both the dispersion relation and the conductance of QRs and thus provides a useful insight for future experimental efforts with regards to transport in QR systems.
\end{abstract}





\section{Acknowledgements}

First, I would like to thank my two supervisors, Prof. Michele Governale and Prof. Ulrich Zülicke, for their patient support and insightful answers to all my questions throughout all the years that we have been working together. In particular, I would like to thank Michele for all the times he's teased me over failing but actually provided me with mental strength to survive this year. I thank Uli for his amazing laughter that reverberates throughout the hallway!

Next, I would also like to thank my colleagues and friends, Markus Kotulla and Kyle Higham, for sitting beside me and sharing the beautiful view over Wellington harbour.

Also, I would like to thank all the students that work at the Fock space (what a smart, yet unfortunate name) for the interesting and much appreciated distractions from work.

Furthermore, I would like to thank my flatmates for putting up with me talking to myself. It has been great to finish my thesis year in a friendly, warm and healthy home environment!

Finally, I would like to thank my family. Especially my step-dad, Petrik Galvosas, who has supported me through my undergraduate and graduate studies by providing me with an endless supply of Kakao, and my partner, Finnian Gray, who, through all the ups and downs, has unwaveringly stood by my side and given me hugs whenever I needed them. 



\section{Contents}

1 Introduction 1

1.1 Motivation of this study . . . . . . . . . . . . . . . 1

1.2 Two-dimensional Dirac-like charge carriers . . . . . . . . 2

1.3 Dirac-like materials . . . . . . . . . . . . . . . . . 5

1.4 Ring confinement of chiral charge carriers . . . . . . . . . . . 7

1.5 Topological insulators . . . . . . . . . . . . . . . . 10 10

1.6 Outline of thesis . . . . . . . . . . . . . . . . . . . . . . 12

2 Methodology 13

2.1 Hamiltonians . . . . . . . . . . . . . . . . . . . . . . . . . 13

2.2 Basic model for ring confinement . . . . . . . . . . . . . . . 18

2.3 Ring-enclosed magnetic (Aharonov-Bohm) flux . . . . . . . . . 22

2.4 Ring confinement with leads . . . . . . . . . . . . . 23

3 Single-layer graphene 27

3.1 Ring structure in SLG . . . . . . . . . . . . . . . . . . . . . . . . 28

$3.1 .1 \quad$ Universal energy curve . . . . . . . . . . . . . . . . . 29 29

3.1 .2 Wavefunctions of SLG . . . . . . . . . . . . 32

3.2 Massless-Dirac case . . . . . . . . . . . . . . . . . . . . . . . . . 33

3.3 General mass-confinement . . . . . . . . . . . . . . . . . . . . 38

$3.3 .1 \quad$ Single-layer graphene . . . . . . . . . . . . . . . . . 38

3.3.2 Transition-metal dichalcogenides . . . . . . . . . . 41

4 Semiconductor quantum well 45

4.1 Ring structures in SQWs . . . . . . . . . . . . . . . . . . . 46

$4.1 .1 \quad$ Secular energy solutions . . . . . . . . . . . . . . . 49

4.1 .2 Transition from topological regime to normal . . . . . . 54

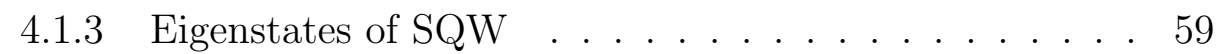

4.2 Finding the azimuthal dependence . . . . . . . . . . . . . 62 
$4.2 .1 \quad$ Magnitude of matrix elements . . . . . . . . . . . 655

$4.2 .2 \quad$ Subband energy dispersion . . . . . . . . . . . 67 67

5 Conductance 75

5.1 General conductance derivation . . . . . . . . . . . 76

5.1 .1 Detection methods . . . . . . . . . . . . . . 8 80

5.2 Massless-Dirac single-layer graphene. . . . . . . . . . . . . . 82

5.3 Transition-metal dichalcogenide . . . . . . . . . . . . . . 86

5.4 Semiconductor quantum well . . . . . . . . . . . . . . . . . . 90 90

6 Geometric Berry phase 97

6.1 Single-layer graphene . . . . . . . . . . . . . . . . . . . . 97

6.2 Semiconductor quantum well . . . . . . . . . . . . . . . . . 100

$6.2 .1 \quad$ Energy-reflection symmetric case . . . . . . . . . . . 100

6.2 .2 Non-energy-reflection symmetric case . . . . . . . . . 106

\begin{tabular}{lll}
\hline 7 & Summary and outlook & 113 \\
\hline
\end{tabular}

A Extra information regarding SQW 119

A.1 Important signs of the SQW model . . . . . . . . . . . . . . . 119

A.2 Comparing our formulation with B. Zhou's . . . . . . . . . . . 119

A.3 General SQW coefficients for wavefunction . . . . . . . . . . . 121

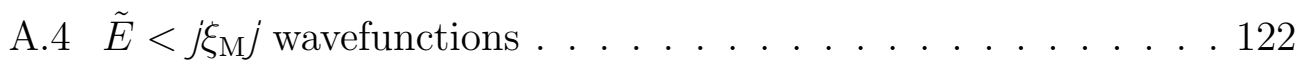

B Useful integrals 


\section{Chapter 1}

\section{Introduction}

Electric current in metals and semiconductors is carried by mobile charge carriers. Normally, the physical properties of such charge carriers are similar to that of electrons at rest in vacuum, except for changes in the magnitude of their mass and magnetic moment. However, within the last $\sim 10$ years, unconventional materials such as two-dimensional atomic crystals (like singlelayer sheets of graphite, called graphene [1]), topological insulators [2], and Weyl semimetals [3] have become available in which charge carriers show quite exotic behaviour. One of these intriguing features is chirality, i.e., the strong coupling of the charge carriers' momentum to their intrinsic magnetic (spin or pseudo-spin) degree of freedom.

Chirality is usually associated with ultra-relativistic fermionic particles such as fast electrons (moving close to the speed of light) or neutrinos which can require vast resources to study experimentally. However, the opportunity to study chirality effects in a condensed matter system makes it possible to access properties that have so far been only theoretically surmised in the context of relativistic quantum mechanics and quantum field theory.

\subsection{Motivation of this study}

In this thesis, we shall theoretically explore confinement effects and transport properties of ring-confined 2D materials using an analytical approach. Many studies employ numerically-based methods to study such properties of these 
systems [4 6]. Others use an analytical procedure to study non-ring specific confinements of materials [7] or focus on a specific material [8]. However, a complete analytical overview of how confinement effects and transport properties vary for different $2 \mathrm{D}$-materials is missing. This is what motivates our research.

Our main focus will be to develop a comprehensive study of quantum confinement effects and transport properties in ring structures of the following 2D materials: single-layer graphene (SLG), single-layer transition-metal dichalcogenides (TMDs) and topological insulators (TIs) made out of narrowgap semiconductor quantum wells (SQWs). Since such materials host chiral charge carriers and are now being experimentally realised [9 14$]$, our theoretical study will provide a timely and fundamental insight to guide further exploration of these systems.

In the following sections of this chapter, we introduce some of the basics that are required to delve deeper into this field. First, we discuss Dirac-like charge carriers and their chiral properties. Then, we give details regarding the structure and key properties of the materials that we wish to explore. Next, the effects of confinement and interference properties of quantum rings and the progress of current research in this area are examined. Following this, we touch upon the nature of topological insulators and topologically protected edge states (TPES). Finally, we outline the general structure of the coming chapters.

\subsection{Two-dimensional Dirac-like charge carri- ers}

Two-dimensional (2D) Dirac-like charge carriers emerge in many condensed matter systems and are described by 2D Dirac-like Hamiltonians. To understand this in more detail, we take a look at the $(\mathrm{D}+1)$-dimensional relativistic Dirac Hamiltonian which is given by

$$
H=\beta m c^{2}+c \boldsymbol{\alpha} \cdot \mathbf{p}
$$


where $c$ is the speed of light, $m$ represents the effective mass, $\mathbf{p}$ is the spatial components of momentum in the $(\mathrm{D}+1)$ dimensions. $\beta$ and $\boldsymbol{\alpha} \equiv\left(\alpha_{1}, \ldots, \alpha_{\mathrm{D}}\right)$ are objects whose forms are fixed by the condition that the Hamiltonian must reproduce the relativistic energy equation

$$
E^{2}=\left(m c^{2}\right)^{2}+(p c)^{2}
$$

where $E$ is the energy. This condition is fulfilled when

$$
\beta^{2}=1 \quad\left\{\alpha_{i}, \beta\right\}=0 \quad \text { and } \quad\left\{\alpha_{i}, \alpha_{j}\right\}=2 \delta_{i j}
$$

where $\{A, B\}=A B+B A$ is the anticommutator.

In $2 \mathrm{D}$, the $\beta$ and $\boldsymbol{\alpha}$ are described by the Pauli matrices. Generally, the most commonly-used representation of the 2D Dirac Hamiltonian is given by:

$$
\mathrm{H}_{2 \mathrm{D}}=c \boldsymbol{\sigma} \cdot \mathbf{p}+V_{0} \sigma_{\mathrm{z}}
$$

where $\boldsymbol{\sigma}=\left(\sigma_{\mathrm{x}}, \sigma_{\mathrm{y}}\right)$ and $V_{0}$ is a potential that can, for example, represent the rest energy $m c^{2}$ for a free relativistic particle or the band-gap in condensed matter systems. In such systems, the wavefunction is represented by a 2 component spinor and the particles that they describe are called Dirac charge carriers.

The general eigenspinor $\Psi(\mathbf{r})$ of the 2D Dirac Hamiltonian is given by

$$
\Psi(\mathbf{r})=\mathbf{N}\left(\frac{1}{\sigma \sqrt{\frac{E-V_{0}}{E+V_{0}}} e^{i \phi_{\mathbf{p}}}}\right) e^{i \mathbf{p} \cdot \mathbf{r} / \hbar}
$$

where $E$ is as given in Eqn. (1.2) [we replace $m c^{2} \rightarrow V_{0}$ ], $\mathrm{N}$ is a normalisation factor, $\sigma \equiv \operatorname{sgn}(E+V(\mathbf{r}))$ and $\phi_{\mathbf{p}}=\arctan \left(p_{y} / p_{x}\right)$. The form of this wavefunction will occur in many chapters to come due to the presence of Dirac-like charge carriers in our materials of interest.

When we replace $c$ by the Fermi velocity $v_{\mathrm{F}}$ in Eqn. (1.4), the 2D Dirac Hamiltonian is able to describe certain condensed-matter systems such as the motion of electrons at high-symmetry points in SLG. Generally, unlike SLG, most condensed matter systems are described by a Dirac-like Hamiltonian (e.g. some systems possess, additionally, spin-splitting). The particles in 
these systems are referred to as Dirac-like charge carriers.

Additionally, in condensed matter systems, two "flavours" (i.e. representations) of the Dirac Hamiltonian are needed to describe the complete lowenergy system, i.e. states with energies close to the Fermi energy. This arises due to the existence of two distinct high-symmetry pseudo-spin valleys that are present in the primitive cell of the reciprocal space, i.e. the Brillouin zone, of the material. Coincidentally, the states in these valleys are time-reversal symmetric to each other. We label these valleys using the label $\tau= \pm 1$ which in our materials refer to the $\mathbf{K}$ and $\mathbf{K}^{\prime}$ valleys, respectively, in SLG \& TMDs or the projection of the angular momentum for $\boldsymbol{\Gamma}$ valley basis states in the growth direction in SQWs. In these systems, the wavefunction is described by the 2-component spinor where the components now correspond to a pseudo-spin degree of freedom.

As mentioned previously, one of the important properties of Dirac charge carriers is their chirality. Chirality is possessed by particles that have a strong coupling between spin (or pseudo-spin) and direction of propagation. This is intriguing because systems with strong chirality present new and unique effects in the presence of a magnetic field such as the anomalous integer quantum Hall effect in graphene 15,16 . The chirality is measured by the chirality operator $h_{\mathrm{c}}$ which is given by:

$$
h_{\mathrm{c}}=\frac{\sigma \cdot \mathrm{p}}{|\mathrm{p}|}
$$

where the eigenvalues of $h_{\mathrm{c}}$ are \pm 1 . When we apply this to the massless Dirac equation (which describes non-confined perfect SLG sheets where $V_{0}=0$ ), this operator commutes with the Hamiltonian [see Eqn. (1.4)] and hence the chirality is exactly known for the energy eigenstates. In the case of SLG, we can choose $\mathbf{H}_{\mathrm{SLG}}^{(\tau)}=\tau v_{\mathrm{F}} \boldsymbol{\sigma} \cdot \mathbf{p} 1$, which describes the charge carriers at both $\tau$ valleys, such that electrons have an $h_{\mathrm{c}}$ eigenvalue equal to $\pm \tau$ where the \pm refers to positive or negative energy eigenvalues, respectively. The chirality on the SLG $\mathbf{K}$ and $\mathbf{K}^{\prime}$ valleys can be seen in Fig. (1.1). This is

\footnotetext{
${ }^{1}$ The particular form of the Hamiltonian depends purely on the choice of basis states which alters none of the physics. Generally in this thesis, we use the SLG representation of the Hamiltonian defined by $\mathrm{H}_{\mathrm{SLG}}^{(\tau)}=v_{\mathbf{F}} \boldsymbol{\sigma}^{*} \cdot \mathbf{p}$ where $\boldsymbol{\sigma}^{*}=\left(\tau \sigma_{\mathrm{x}}, \sigma_{\mathrm{y}}\right)$. However, we use the alternative representation here to demonstrate chirality concepts because this choice of basis provides a simpler demonstration of the main ideas.
} 
just one demonstration of the strong chiral nature that is present in many condensed-matter systems. In the next section we will delve into the structural properties of the $2 \mathrm{D}$ materials that are of interest to us.

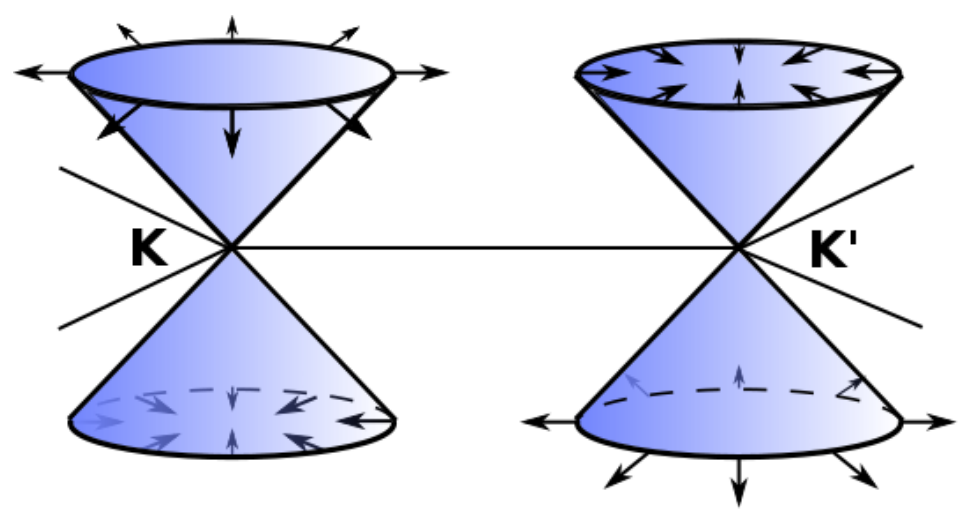

Figure 1.1: Pseudo-spin chirality (indicated by the arrows) of $\mathbf{K}$ and $\mathbf{K}^{\prime}$ Dirac cones in graphene where the chirality eigenvalue is equal to $\pm \tau$ for positive and negative energies, respectively. Chiral charges exhibit interesting properties due to their strong spin-orbit coupling.

\subsection{Dirac-like materials}

Single-layer graphene consists of a plane of carbon atoms in a hexagonal (or honeycomb) lattice as depicted in Fig. (1.2a). The two-component spinor arises due to the presence of a degree of freedom (i.e. pseudo-spin), emerging from the existence of two identical atoms (sublattice-sites A and B) in the unit cell. One of the exotic properties of a perfect SLG sheet (where $V_{0} \rightarrow$ 0 ) is that for low energies, it possesses a linear energy dispersion that is centred at the high-symmetry points $[17,18$. This feature is much akin to that of free massless, relativistic particles such as the neutrino. Thus we can potentially observe ultra-relativistic behaviour on a SLG "chip". This feature, in addition to its strong chiral properties and simple Hamiltonian, is the main reason for our interest in it.

Now we turn to present the basic properties of single-layer transition-metal dichalcogenides. TMDs possess three atomic layers constructed in the form of $\mathrm{X}-\mathrm{M}-\mathrm{X}[\mathrm{M}=$ molybdenum (Mo) or tungsten $(\mathrm{W})$ and $\mathrm{X}=\operatorname{sulphur}(\mathrm{S})$, selenium (Se) or tellurium (Te)] as depicted in Fig. (1.2b) [19]. However, 
viewed from the top, similarly to graphene, a 2D hexagonal-shaped lattice of alternating $\mathrm{X}_{2}$ (the 2 refers to two $\mathrm{X}$ atoms that are projected to the same point) and $\mathrm{M}$ atoms exists, as can be seen in Fig. (1.2). In this case, the two-component spinor represents the degree of freedom corresponding to the conduction and valence band. In addition to the properties that are present in SLG (i.e. strong chirality), TMDs also possess a direct band gap with strong real-spin-orbit coupling (leading to the spin-splitting) around their $\mathbf{K}$ and $\mathbf{K}^{\prime}$ valleys for low energies. The real-spin splitting is particularly prominent in the valence band [19]. Due to these additional features, TMDs will allow us to deepen our understanding of confinement effects in more complicated systems.

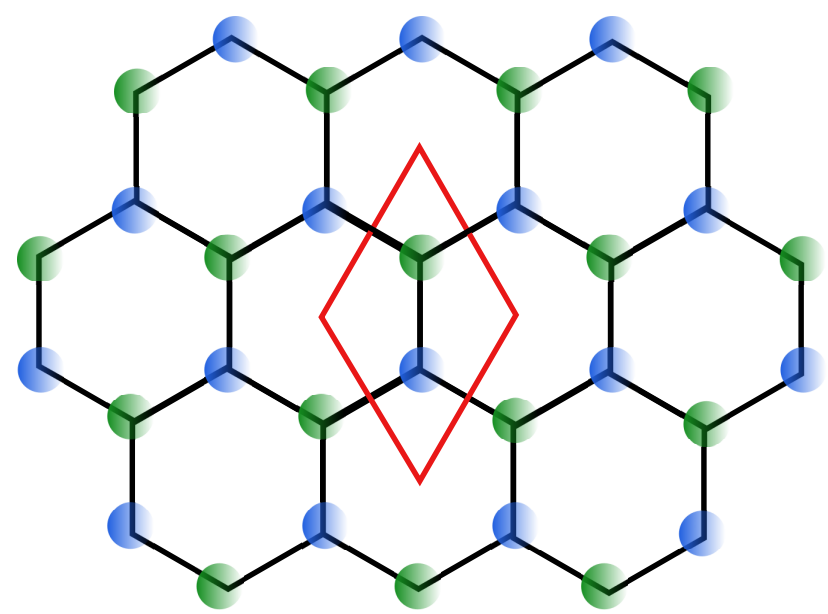

(a) Honeycomb lattice

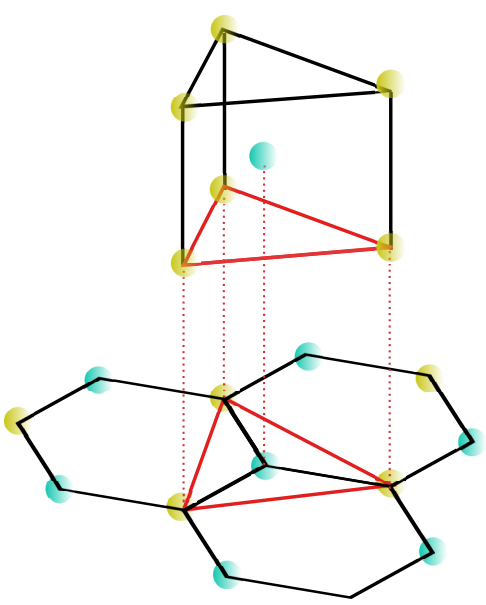

(b) TMD layer structure

Figure 1.2: (a) The general honeycomb lattice that is present in both SLG and TMDs. The green and blue circles represent the carbon sublattice sites $A$ and $B$, respectively, in $S L G$ and alternating chalcogenide (X) and transitionmetal atoms $(\mathrm{M})$, respectively, in TMD. The red diamond represents the smallest unit cell. (b) Layer structure of TMD is visible which, when projected down from top-view, results in a honeycomb lattice. Here, yellow represents the $\mathrm{X}$ atoms and blue represents the $\mathrm{M}$ atoms.

The final material that we are interested in studying is the semiconductor quantum well [e.g. mercury telluride (HgTe) quantum well [20] or indium arsenide and gallium antimonide (InAs/GaSb) quantum well [21]]. These materials are essentially a sandwich of one sheet of semiconductor $(\mathrm{HgTe})$ between two other sheets of semiconductors [cadmium telluride (CdTe)] which can be seen in Fig. 1.4. The CdTe layers have a wider band-gap than 
the HgTe layer and hence act as insulating barriers that create discrete energy subbands within HgTe. The two-component spinors for HgTe quantum wells represent the spin-quantum number $m_{s}= \pm\left(\frac{1}{2}, \frac{3}{2}\right)$ at the relevant highsymmetry valley $(\boldsymbol{\Gamma})$ and hence are pseudo-spins [2,22]. Unlike SLG or TMD, SQWs have only one high-symmetry valley in the Brillouin zone with two distinct Dirac cones centred on it. SQWs also have a band-gap and dependences on $\mathbf{p}^{2}$ terms making them much more complex compared to SLG. Importantly, some SQWs (such as HgTe quantum wells) possess a tuneable and invertible band-gap which would permit experimentalists to explore the confinement-related changes of the topological nature of SQWs ${ }^{2}$. Changing the band-gap is much more difficult in the cases of SLG and TMDs.

Many of the interesting effects arising from chirality manifest themselves in structures where the motion of the charge carriers is confined, e.g., to a narrow (quantum) wire or a quantum ring. Hence, we proceed to the next section where we consider general phenomena that may arise when we confine chiral charge carriers in quantum rings.

\subsection{Ring confinement of chiral charge carri- ers}

Quantum rings (QRs) are generally defined as nanoscale sized doubly connected ring-like structures 23] [a diagram of a SLG ring can be seen in Fig. (1.3)]. They are especially interesting structures because they are a paradigm for investigating particle-wave duality and other quantum-physical effects. This is possible due to the practical detection of changes to physical observables when particles travel down two different branches of the ring and interfere upon recombination. In particular, QRs present an opportunity to observe how geometric phases (also known as Berry phases) affect physical quantities.

Geometric phases are generally phase differences between the initial and final eigenstates. One of the most prominent examples of a geometric phase is the famous Aharanov-Bohm effect $24-26]$ whereby the magnetic flux that

\footnotetext{
${ }^{2}$ We will discuss this in more detail in Sec. 1.5 .
} 
threads through the centre of a ring [see setup in Fig. (1.3)] will modulate the transmission through the ring. To understand this, we begin with the wavefunction at a point $\mathbf{r}$ in the presence of a magnetic vector potential $\mathbf{A}$ which is given by

$$
\Psi=\Psi_{0} e^{i \varphi(\mathbf{r})} \quad \text { where } \quad \varphi(\mathbf{r})=\frac{e}{\hbar} \int_{\mathbf{r}_{0}}^{\mathbf{r}} \mathbf{A}\left(\mathbf{r}^{\prime}\right) \cdot d \mathbf{r}^{\prime}
$$

Here $\Psi_{0}$ is the wavefunction when $\mathbf{A}=0$ and $\mathbf{r}_{\mathbf{0}}$ is an arbitrary initial point where the magnetic field $\mathbf{B}=0$. In simply connected regions of space where $\mathbf{B}=\boldsymbol{\nabla} \times \mathbf{A}=0$, the line integral only depends on the beginning and end points. Thus, when we calculate the probability of finding a particle in the region of interference between partial waves travelling above, $\Psi_{\text {above }}$, and below, $\Psi_{\text {below }}$, the magnetic flux through the ring, we have

$$
\left|\Psi_{\text {above }}+\Psi_{\text {below }}\right|^{2} \propto 1+\cos (\gamma)
$$

where

$$
\gamma=\frac{e}{\hbar}\left[\int_{\mathbf{r}_{\mathbf{0}}}^{\mathbf{r}} \mathbf{A}_{\text {above }} \cdot d \mathbf{r}^{\prime}-\int_{\mathbf{r}_{\mathbf{0}}}^{\mathbf{r}} \mathbf{A}_{\text {below }} \cdot d \mathbf{r}^{\prime}\right]=\frac{e}{\hbar} \oint \mathbf{A} \cdot d \mathbf{r}^{\prime}=2 \pi \frac{\phi}{\phi_{0}}
$$

where $\phi$ is the magnetic flux through the centre of the ring and $\phi_{0}=$ $2 \pi \hbar / e$ [26]. To obtain the last equality we have used Stokes theorem to turn the line integral into a surface integral representing the flux. Hence, the conductance of such a system becomes periodic as a function of $\phi / \phi_{0}$.

Another simple (and relevant) demonstration of a Berry phase is when a particle circles around a magnetic field and gains a phase factor that is proportional to $e^{-i m \Omega}$ where $m$ is the particle's spin component along the magnetic field and $\Omega$ is the angle subtended by the circuit around the magnetic field [27]. When the particle is a fermion (i.e. possesses a half-integer spin), a full circuit around the magnetic field produces a non-trivial phase of -1 . Both these above mentioned geometric phases will play a crucial role in the understanding of ring transport properties of chiral charge carriers in the presence of a magnetic field.

The essence of these examples is that the Berry phase, although "just" a phase difference between the initial and final wavefunctions, can also manifest 


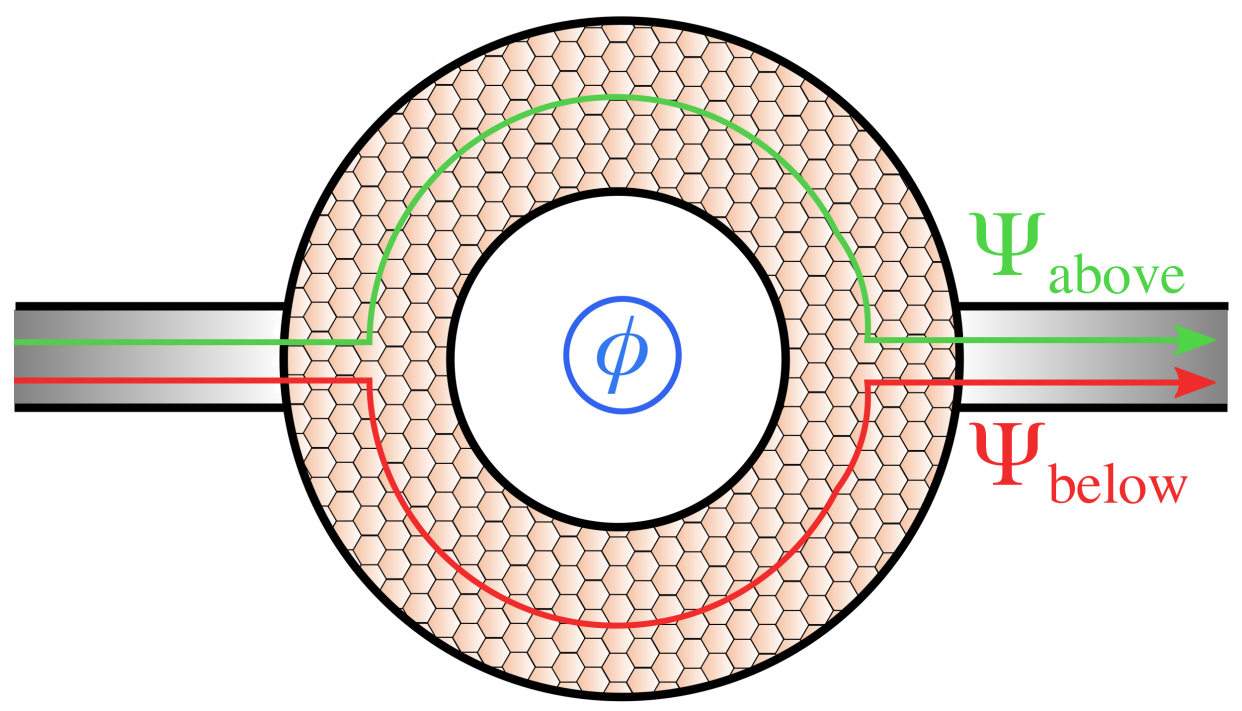

Figure 1.3: Schematic setup of a graphene ring (orange) with leads (grey) attached. To demonstrate the Aharanov-Bohm setup, we have threaded the ring with a magnetic flux $\phi$ (i.e. the magnetic field is perpendicular to the plane of the ring). The partial waves $\Psi_{\text {above }}$ and $\Psi_{\text {below }}$ travel via different branches of the ring and eventually interfere in the lead on the right.

itself in physical observables such as in the conductivity.

In particular, ring structures of graphene, TMDs and topological insulators are scientifically desirable to study due to the possibility of detecting the effects of exotic interplay between the Dirac-like charge carriers and the confinement of the ring. This could yield unique geometric phases. The strong presence of chirality allows for phenomena such as the $\pi$ Berry phase in SLG, TMD and SQW to arise [28,29] due to the rotation of the pseudospin with the electron's momentum. In addition, having chiral charge carriers enables the design of novel electronic devices that could become useful for quantum information processing and spintronics devices in the realm of nanoelectronics 23, 30 33.

Fabrication of general 2D quantum rings started around the mid-eighties with the endeavour to realise smaller and smaller rings [4]. The first example of a laboratory made graphene ring was first constructed in 2008 by S. Russo et. al. 99. The fabrication of HgTe QRs has also been realised [34].

The theoretical treatment of quantum rings still centres around some corner- 
stone papers that were published slightly before the fabrication of QRs. The conductance of quantum rings with leads was a central focus of a variety of studies in the mid-eighties [35 37]. They modelled scattering through leads using a scattering matrix and derived expressions for the conductance. We utilise these results to understand conductances and transport properties of chiral charges in our systems.

Finally, one of the other intriguing feature of ring confinement is that it causes changes in the topological nature of certain materials. This happens because decreasing the width increases confinement energies and thus may result in states that were initially topologically protected (i.e. they are robust to time-reversal symmetric impurities) to become trivial [38,39]. To understand this we move to the next section in which we give a brief overview of the topological nature of TIs.

\subsection{Topological insulators}

The defining feature of a topological insulator is the presence of an inverted band gap which allows for the existence of topologically protected edge states (TPES) within the insulating band gap. TPES are not affected by timereversal symmetric perturbations and hence are desirable to study in the realm of quantum computing [40,41] and the fabrication of devices such as topological spin transistors 42 .

HgTe quantum wells, as depicted in Fig. 1.4a), are generally (dependent on system parameters) a type of $2 \mathrm{D}$ topological insulator due to the presence of an invertible band gap. This is possible due to the strong spin-orbit coupling in $\mathrm{Hg}$ which causes it to have an inverted band structure [2]. As mentioned in Sec. (1.3), HgTe-based quantum wells also have the advantage of being able to tune the gap parameter by changing the size of the quantum well [20]. This is because the decrease of the width, $d$, increases the confinement effects thus forcing an inverted gap to decrease and eventually become normal at a critical width $d_{c}$. This is illustrated in Fig. (1.4b).

Hence, due to these properties, one can easily manipulate HgTe quantum well system parameters to alter the topological nature of the material. When additionally confined in a ring, we gain a second width parameter, $W$, which 
we expect to have similar effects on the topological nature. If this is the case, we gain fundamental insight into quantum confinement effects in topological insulators.

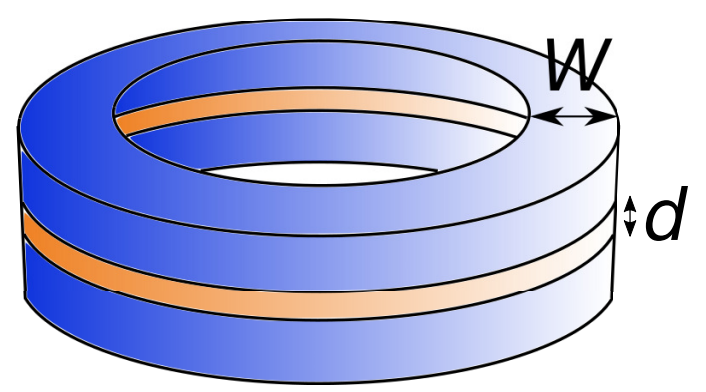

(a) HgTe quantum well ring
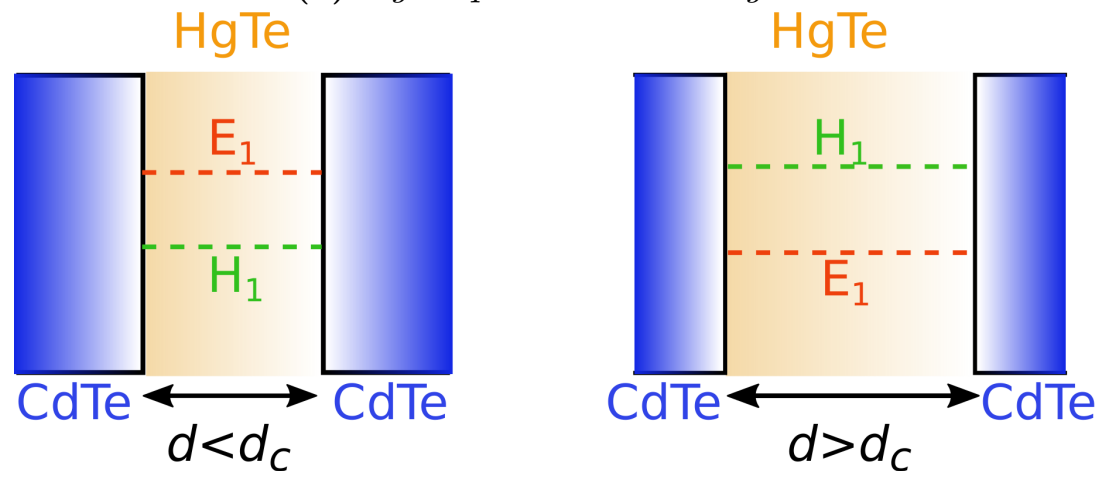

(b) HgTe quantum well band inversion at $d_{c}$

Figure 1.4: (a) Schematic setup of an HgTe quantum well ring where the HgTe layer (orange) is sandwiched between the CdTe layers (blue). (b) As we go from $d<d_{c}$ to $d>d_{c}$ the band becomes inverted with light-hole (or heavy-hole) band $H_{1}$ moving to be above electron band $E_{1}$ thus causing band inversion. The band gap is measured as $M \equiv E_{1}-H_{1}$. This figure is adapted from Ref. [20].

Another distinguishing feature between topologically trivial and non-trivial insulators lies in the number of pairs of time-reversal symmetric states (i.e. Kramers pairs) at a given energy in the gap: there exist either an even or odd amount of these pairs. Topological insulators possess an odd number of Kramer pairs [43]. Since time-reversal symmetric impurities destroy an even set of Kramers pairs, TIs retain at least one pair of Kramer pairs. Thus, this always guarantees that the insulator stays in the topological phase and hence we call bound states in TIs topologically protected.

Additionally, TIs can be assigned a distinct $\mathbb{Z}_{2}$ topological invariant that 
differs from that of a trivial insulator 3 - this invariant can be understood to be similar to the difference between a sphere and a torus: neither can be deformed to the other due to their distinct topologies. For further details, the reader is referred to Ref. [44].

\subsection{Outline of thesis}

In this thesis we apply modifications of a general, overarching methodology to analyse the specific materials: single-layer graphene, transition-metal dichalcogenides and narrow-gap semiconductor wells (such as HgTe quantum wells).

First we lay out the methodology used to examine the confinement properties and conductances of SLG, TMD and SQW QR systems in Chapter 2.

In Chapter 3, we apply this methodology to discover the confinement properties of SLG and determine the general subband energy dispersion amongst other results. Since TMDs possess an identical Hamiltonian as SLG with the addition of an energy shift due to spin splitting, they are also included in this chapter. In Chapter 4 we apply a similar procedure to SQW.

In the next two chapters physical observables of $\mathrm{QRs}$, in the presence of a magnetic field, are examined. First in Chapter 5, we investigate the conductance properties of QRs with leads for the relevant materials. Then in Chapter 6, we study the confinement-induced geometric phases of these materials and the connection to their topological phase.

Finally in Chapter 7, we review our results, compare to previous literature and discuss the implications of our findings. We conclude by suggesting possible directions for future work.

\footnotetext{
${ }^{3}$ The elements of the group $\mathbb{Z}_{2}$ are $\{0,1\}$ 44. Trivial and topological insulators are assigned the index 0 and 1 respectively hence distinguishing them.
} 


\section{Chapter 2}

\section{Methodology}

In this chapter, we lay down the basic methodology that is used to analyse ring-confinement and transport properties of SLG, TMD and SQW QR systems.

First, we present the low-energy effective $\mathbf{k} \cdot \mathbf{p}$ Hamiltonians, $\mathbf{H}_{\alpha}^{(\tau)}$, for each material (labelled by $\alpha$ ) with an explanation of relevant terms and regimes of applicability. Then, a radially-symmetric ring-confinement model is applied to the Hamiltonians resulting in the possibility to split them into a radial, $H_{\alpha, 0}^{(\tau)}$, and azimuthal component, $H_{\alpha, 1}^{(\tau)}$. Following this, we assume knowledge of eigenstates and energies of $H_{\alpha, 0}^{(\tau)}$, which we use to transform both $H_{\alpha, 0}^{(\tau)}$ and $H_{\alpha, 1}^{(\tau)}$ into a new basis thus giving $\mathrm{H}_{\alpha, 0}^{(\tau)}$ and $\mathrm{H}_{\alpha, 1}^{(\tau)}$, respectively. Using perturbation theory that ignores inter-subband interactions, we solve for the general subband dispersion relation of the system. Then, we outline the changes in the system in the presence of a magnetic field. Finally, we discuss the scattering model that is used to find transport properties in QRs with leads.

\subsection{Hamiltonians}

We are interested in the behaviour of electrons at low-energies close to the high-symmetry valleys of our 2D-Dirac-like materials. In this regime, their motion can be described by a low-energy effective $\mathbf{k} \cdot \mathbf{p}$ Hamiltonian. The $\mathbf{k} \cdot \mathbf{p}$ method uses perturbation theory and semi-empirical data to discard higher 
order $\mathbf{k}$ terms thus leading to a simplified band dispersion [45, 46]. For all of our materials, we present the Hamiltonians in terms of an equation that describes both "flavours" of electrons using the label $\tau \equiv \pm 1$. The $\tau$ index labels subspaces that are linked by time-reversal symmetry. To reiterate from Chapter 1, these different flavours are necessary since they describe charge carriers at different high-symmetry points in the Brillouin zone of the material.

It is well-known that any type of mass gap, either intrinsic [47] or confinementinduced [5, 48], causes dynamics that are only time-reversal invariant if both flavours of 2D-Dirac electrons are included. We therefore discuss the effect of ring confinement consistently for both. However, we neglect processes that couple the time-reversal-related flavors, thus assuming graphene and dichalcogenide rings to be sufficiently wide and point-defect-free, and spinsplitting in SQWs to be absent. In the following section, we present the forms of the Hamiltonians that are used and touch upon crucial concepts for each case.

\section{Single-layer graphene}

For SLG, we use the standard Hamiltonian which is usually derived using the nearest-neighbour tight-binding method and identical to results from the $\mathbf{k} \cdot \mathbf{p}$ method [18]. The Hamiltonian is expanded around the high-symmetry points $\mathbf{K}$ and $\mathbf{K}^{\prime}$ in the Brillouin zone to obtain two distinct equations for the corresponding valleys ( $\tau= \pm 1$ respectively) using the pseudo-spin basis corresponding to the sublattice sites A and B: $\left\{\left|A \mathbf{i}^{(\tau)},\right| B \mathbf{i}^{(\tau)}\right\}$. The resulting Hamiltonian can be shown to be:

$$
\mathrm{H}_{\mathrm{SLG}}^{(\tau)}=\hbar v \tau\left(k_{\tau} \sigma_{-}+k_{-\tau} \sigma_{+}\right)
$$

where $\hbar$ is the reduced Planck constant, $v$ is the Fermi velocity, $\sigma_{ \pm}=$ $\left(\sigma_{1} \pm i \sigma_{2}\right) / 2$ are pseudo-spin-1/2 ladder operators for the eigenstates of $\sigma_{3} \equiv \operatorname{diag}(1,-1)$ that correspond to the Bloch-function basis at $\mathbf{K}$ and $\mathbf{K}^{\prime}$ points, and $\sigma_{0}$ is the identity matrix in pseudo-spin space. Additionally, $k_{ \pm}:=k_{x} \pm i k_{y}$ is in terms of the Cartesian components of the in-plane wave vector $\mathbf{k}$. A limitation of this model is the fact that we ignore all interac- 
tions other than those from the nearest neighbours 1 . Generally, since we stay in the low-energy regime, these contributions from further neighbours are negligible since the coupling decreases with the distance of separation.

\section{Single-layer transition-metal dichalcogenides}

For single-layer transition-metal dichalcogenides, we use the model that was introduced by D. Xiao et. al. [49]. Using the $\mathbf{k} \cdot \mathbf{p}$ method, this Hamiltonian is derived via symmetry argument applied to the simple minimal band model. A spin-orbit coupling term is present due to the presence of spin-splitting in the valence band. The basis used for the Hamiltonian consists of the conduction band (CB) and valence band (VB) and real spin $s$ : $\left\{\left|\mathrm{CB}, s \mathbf{i}^{(\tau)},\right| \mathrm{VB}, s \mathbf{i}^{(\tau)}\right\}$. For $\mathrm{MoS}_{2}$, the individual elements are given by

$$
\begin{aligned}
& \left|\mathrm{CB}, s \mathbf{i}^{(\tau)}=\right| d_{z^{2}} \mathbf{i} \\
& \mid \mathrm{VB}, s \mathbf{i}^{(\tau)}=\frac{\sqrt{ }}{\overline{2}}\left(\left|d_{x^{2}-y^{2}} \mathbf{i}+i \tau\right| d_{x y} \mathbf{i}\right)
\end{aligned}
$$

where $d$ is an orbital that splits into three groups (for more details, please refer to [49]). Although this is specifically for $\mathrm{MoS}_{2}$, the physics, Hamiltonian and basis are essentially the same for all group-VI dichalcogenides. The general Hamiltonian is thus given by

$$
\mathrm{H}_{\mathrm{TMD}}^{(\tau)}=\hbar v \tau\left(k_{\tau} \sigma_{-}+k_{-\tau} \sigma_{+}\right)+\frac{\Delta}{2} \sigma_{3}-\lambda \tau s_{3} \frac{\sigma_{3}-\sigma_{0}}{2}
$$

where $\Delta$ is the band-gap energy and $\lambda$ is the strength of the spin-splitting in the valence band, $s_{3}$ denotes the Pauli matrix for the real spin of electrons in $\mathrm{TMD}^{2}$ and all the other parameters are the same as in the SLG case. The only difference between this Hamiltonian and that of SLG is the band gap and the valence-band spin-splitting term. Naturally, as in the SLG case, the model that we use for TMD is a simplification of the general system. A more general approach may involve implementing the seven-band $\mathbf{k} \cdot \mathbf{p}$ model

\footnotetext{
${ }^{1}$ Coincidentally, it so happens that the contributions from next-nearest neighbours just shift the energy at the $\mathbf{K}$ and $\mathbf{K}^{\prime}$ valleys and thus, without loss of generality, can be set to zero.

${ }^{2}$ Notice that the real spin $s_{3}$ is completely decoupled for spin up and down. However, this still differs to the SLG system where energies are degenerate for real spin $\uparrow$ and $\downarrow$.
} 
that takes into account more energy bands and additional degrees of freedom in the system $19,50,51$. However, this is not necessary in the low-energy regime since we focus on the nearest bands to the $\mathbf{K}$ and $\mathbf{K}^{\prime}$ points.

\section{Semiconductor quantum wells}

In the case of SQWs, specifically HgTe quantum wells, we use the simple model first put out by B. A. Bernevig et. al. [20]. The Hamiltonian described the movement of charge carriers around the $\boldsymbol{\Gamma}$ high-symmetry Brillouin zone point. Using $\mathbf{k} \cdot \mathbf{p}$ symmetry arguments regarding the coupling of basis $\left\{\left|E_{1}+\mathbf{i},\right| H_{1}+\mathrm{i}\right\}$ for $\tau=1$ and $\left\{\left|E_{1}-\mathbf{i},\right| H_{1}-\mathbf{i}\right\}$ for $\tau=-1$ the Hamiltonian is deduced. The basis elements are given by

$$
\begin{aligned}
& \left|E_{1} \pm \mathbf{i}=\alpha\right| \Gamma_{6}, \pm \frac{1}{2} \mathbf{i}+\beta \mid \Gamma_{8}, \pm \frac{1}{2} \mathbf{i} \\
& \left|H_{1} \pm \mathbf{i}=\right| \Gamma_{8}, \pm \frac{3}{2} \mathbf{i}
\end{aligned}
$$

where $\alpha$ and $\beta$ are just constants. Thus for HgTe quantum wells, $\tau$ corresponds to the projection of total angular momentum for $\boldsymbol{\Gamma}$-point basis states in the growth direction. The SQW Hamiltonian is given by

$$
\mathrm{H}_{\mathrm{SQW}}^{(\tau)}=\hbar v \tau\left(k_{\tau} \sigma_{-}+k_{-\tau} \sigma_{+}\right)+\frac{\Delta(k)}{2} \sigma_{3}+\epsilon_{k} \sigma_{0}
$$

where we use the usual parametrizations [20,52] $\epsilon_{k}=C-D k^{2}$ and $\Delta(k) / 2=$ $M-B k^{2}$. The material parameters $M, B, C$ and $D$ are determined by the setup of the system (e.g. width of SQW etc.). In this case, the eigenstates of $\sigma_{3}$ correspond to the Bloch-function basis at the $\boldsymbol{\Gamma}$-point. In the following, and without loss of generality, we set $C=0$ for convenience since it is just a constant energy shift. It is of importance to note that the $M$ parameter is the gap parameter that causes band inversion and is determined by the width of the middle (e.g. HgTe) layer in the SQW [as explained and shown in Fig. (1.4b)]. Naturally, this model is a simplification that ignores bulk inversion asymmetry which couples the different $\tau$ flavours. This becomes important for finding the exact spin orientation of edge states and can be taken account of by adding the $\mathrm{H}_{\mathrm{BIA}}$ term, as given in Refs. [2, 52, to the $\mathbf{H}_{\mathrm{SQW}}^{(\tau)}$. However, this is not within the frame of interest with respect to 
confinement-effect in QR systems and thus we proceed with the simplified model.

\section{Polar coordinate transformation}

Since we work with a ring geometry and an axially symmetric confinement, it is only natural for us to express the Hamiltonians in polar coordinates $\mathbf{r}=(r, \varphi)$. It is helpful to note the relation

$$
k_{ \pm}=\mathrm{e}^{ \pm i \varphi / 2}\left(k_{r} \pm i k_{\varphi}\right) \mathrm{e}^{ \pm i \varphi / 2}
$$

with the Hermitian operators for radial and azimuthal wave-vector components

$$
\begin{aligned}
& k_{r}=-i\left(\partial_{r}+\frac{1}{2 r}\right) \\
& k_{\varphi}=-i \frac{\partial_{\varphi}}{r}
\end{aligned}
$$

For later reference, we also note the identities

$$
\begin{aligned}
k_{r}^{2} & =-\partial_{r}^{2}-\frac{1}{r} \partial_{r}+\frac{1}{4 r^{2}}, \\
\left\{k_{r}, k_{\varphi}\right\} & \equiv k_{r} k_{\varphi}+k_{\varphi} k_{r}=-\frac{2}{r} \partial_{r} \partial_{\varphi}, \\
\left\{k_{r}, 1 / r\right\} & =-i \frac{2}{r} \partial_{r} .
\end{aligned}
$$

It is then straightforward to apply these coordinate transformations to Eqns. 2.1), (2.4) and (2.7) and obtain

$$
\begin{aligned}
\mathbf{H}_{\mathrm{SLG}}^{(\tau)} & =\mathbf{U}_{\tau} \hbar v\left(k_{r} \tau \sigma_{1}+k_{\varphi} \sigma_{2}\right) \mathbf{U}_{\tau}^{\dagger}, \\
\mathbf{H}_{\mathrm{TMD}}^{(\tau)} & =\mathbf{U}_{\tau}\left[\hbar v\left(k_{r} \tau \sigma_{1}+k_{\varphi} \sigma_{2}\right)+\frac{\Delta}{2} \sigma_{3}-\lambda \tau s_{3} \frac{\sigma_{3}-\sigma_{0}}{2}\right] \mathbf{U}_{\tau}^{\dagger}, \quad \text { (2.11b) } \\
\mathbf{H}_{\mathrm{SQW}}^{(\tau)} & =\mathbf{U}_{\tau}\left[\hbar v\left(k_{r} \tau \sigma_{1}+k_{\varphi} \sigma_{2}\right)-\left(k_{r}^{2}+k_{\varphi}^{2}\right)\left(D \sigma_{0}+B \sigma_{3}\right)+M \sigma_{3}\right] \mathbf{U}_{\tau}^{\dagger} .
\end{aligned}
$$


where $\bigcup_{\tau}(\varphi)=\exp \left\{-i \tau \frac{\sigma_{3}}{2} \varphi\right\}$. These expressions form the basis for our further study of quantum states in ring conductors.

\subsection{Basic model for ring confinement}

We assume the ring structure to be defined by an axially symmetric mass confinement $V(r) \sigma_{3}$. A mass confinement $\left(\sigma_{3}\right.$ instead of $\left.\sigma_{0}\right)$ is used because it applies the same confinement-potential on particles of the same mass, thus treating electrons and holes the same [53]. This gives rise to the Schrödinger equation

$$
\left[\mathbf{H}_{\alpha}^{(\tau)}+V(r) \sigma_{3}\right]\left|\Psi_{\alpha}^{(\tau)} \mathbf{i}=E_{\alpha}^{(\tau)}\right| \Psi_{\alpha}^{(\tau)} \mathbf{i}
$$

where $\alpha=$ SLG, TMD, SQW. We make the Ansatz

$$
\left|\Psi_{\alpha}^{(\tau)} \mathbf{i}=\bigcup_{\tau}(\varphi) \mathrm{e}^{i l \varphi}\right| \Phi_{l, \alpha}^{(\tau)} \mathbf{i}
$$

where $\hbar l$ is the eigenvalue of $r k_{\varphi}$ (the $z$ component of total angular momentum). This transforms Eqn. (2.12) into the unitarily equivalent problem

$$
\left[H_{\alpha, 0}^{(\tau)}+H_{\alpha, 1}^{(\tau)}(l)\right]\left|\Phi_{l, \alpha}^{(\tau)} \mathbf{i}=E_{\alpha}^{(\tau)}(l)\right| \Phi_{l, \alpha}^{(\tau)} \mathbf{i} \quad
$$

where $H_{\alpha, 0}^{(\tau)}$ is purely radial and particle-hole symmetric $3^{3}$, and $H_{\alpha, 1}^{(\tau)}(l)$ contains all the terms associated with the azimuthal motion and any (typically very small) particle-hole-symmetry-breaking terms. For the cases of interest here, we find the following expressions for the corresponding parts of the Hamiltonian:

(i) Single-layer graphene:

$$
\begin{aligned}
H_{\mathrm{SLG}, 0}^{(\tau)} & =\hbar v k_{r} \tau \sigma_{1}+V(r) \sigma_{3} \\
H_{\mathrm{SLG}, 1}^{(\tau)} & =\hbar v \frac{l}{r} \sigma_{2} .
\end{aligned}
$$

(ii) Single-layer transition-metal dichalcogenides: because of their fundamental similarity, we can combine all terms involving $\sigma_{3}$ into a new effective

\footnotetext{
${ }^{3}$ Actually, it is the energy-reflection symmetry that we need. See Ref. 54] for more details of discrete symmetries exhibited by Dirac models.
} 
potential $\tilde{V}(r) \equiv V(r)+\Delta / 2-\lambda \tau s_{3} / 2$, thus obtaining

$$
\begin{aligned}
& H_{\mathrm{TMD}, 0}^{(\tau)}=\hbar v k_{r} \tau \sigma_{1}+\tilde{V}(r) \sigma_{3} \\
& H_{\mathrm{TMD}, 1}^{(\tau)}=\hbar v \frac{l}{r} \sigma_{2}+\frac{\lambda}{2} \tau s_{3} \sigma_{0}
\end{aligned}
$$

Except for the particle-hole-asymmetric contribution to $H_{\mathrm{TMD}, 1}^{(\tau)}$, this Hamiltonian is in essence formally identical to SLG.

(iii) Narrow-gap semiconductor quantum well: we can again combine $M$ and the confinement into the effective potential $\tilde{V}(r) \equiv V(r)+M$. The SQW QR Hamiltonian then becomes

$$
\begin{aligned}
& H_{\mathrm{SQW}, 0}^{(\tau)}=\hbar v k_{r} \tau \sigma_{1}-B k_{r}^{2} \sigma_{3}+\tilde{V}(r) \sigma_{3}-D k_{r}^{2} \sigma_{0} \\
& H_{\mathrm{SQW}, 1}^{(\tau)}=\hbar v \frac{l}{r} \sigma_{2}-\frac{l^{2}}{r^{2}}\left(B \sigma_{3}+D \sigma_{0}\right) .
\end{aligned}
$$

Here we have departed from the general philosophy by including a particlehole-asymmetric contribution in $H_{\mathrm{SQW}, 0}^{(\tau)}$ (i.e. the $\sigma_{0}$ term). This turns out to be necessary because $|D| \lesssim|B|$ is typically not a small correction [52]. As a result, some straightforward modifications will have to be made when applying the formalism developed below to the SQW case.

We proceed by considering the radial Schrödinger equation

$$
H_{\alpha, 0}^{(\tau)}\left|\phi_{\alpha}^{(\tau, n)} \mathbf{i}=E_{\alpha, 0}^{(\tau, n)}\right| \phi_{\alpha}^{(\tau, n)} \mathbf{i}
$$

with its set of discrete energy eigenvalues $E_{\alpha, 0}^{(\tau, n)}$ and corresponding eigenstates $\mid \phi_{\alpha}^{(\tau, n)} \mathbf{i}$, and $n= \pm 1, \pm 2, \ldots$ In practice, to solve for the eigenvalues and eigenstates, we map the Schrödinger equation into a 1D Dirac-like equation which is possible due to the symmetry of the ring system. By our explicit construction (except in the SQW case which can be easily addressed), the energy-reflection symmetry 54

$$
\sigma_{2} H_{\alpha, 0}^{(\tau)} \sigma_{2}=-H_{\alpha, 0}^{(\tau)}
$$

holds, which implies $E_{\alpha, 0}^{(\tau,-n)}=-E_{\alpha, 0}^{(\tau, n)}$ and $\left|\phi_{\alpha}^{(\tau,-n)} \mathbf{i}=\sigma_{2}\right| \phi_{\alpha}^{(\tau, n)} \mathbf{i}$. From now on, we thus assume without loss of generality $n>0$ and $E_{\alpha, 0}^{(n)}>0$.

In the spirit of subband $\mathbf{k} \cdot \mathbf{p}$ theory [55, 56], we now posit that the radial 
Schrödinger equation 2.18 has been solved and make the Ansatz

$$
\mid \Phi_{l, \alpha}^{(\tau, \pm n)} \mathbf{i}=\sum_{n^{\prime}>0}\left(a_{l, \tau, \alpha}^{\left( \pm n, n^{\prime}\right)}\left|\phi_{\alpha}^{\left(\tau, n^{\prime}\right)} \mathbf{i}+b_{l, \tau, \alpha}^{\left( \pm n, n^{\prime}\right)}\right| \phi_{\alpha}^{\left(\tau,-n^{\prime}\right)} \mathbf{i}\right)
$$

for the general eigenstates. The unknown coefficients in this expansion are then found from the discrete matrix-eigenvalue problem

$$
\left[\mathrm{H}_{\alpha, 0}^{(\tau)}+\mathrm{H}_{\alpha, 1}^{(\tau)}\right]\left(\begin{array}{c}
a_{l, \tau, \alpha}^{( \pm n, 1)} \\
b_{l, \tau, \alpha}^{( \pm n, \alpha)} \\
a_{l, \tau, \alpha}^{( \pm n, 2)} \\
b_{l, \tau, \alpha}^{( \pm n, 2)} \\
\vdots
\end{array}\right)=E_{\alpha}^{(\tau, n)}(l)\left(\begin{array}{c}
a_{l, \tau, \alpha}^{( \pm n, 1)} \\
b_{l, \tau, \alpha}^{( \pm n, 1)} \\
a_{l, \tau, \alpha}^{( \pm n, 2)} \\
b_{l, \tau, \alpha}^{( \pm n, 2)} \\
\vdots
\end{array}\right)
$$

with the new Hamiltonian matrices

$$
\mathbf{H}_{\alpha, j}^{(\tau)}=\left(\begin{array}{ccccc}
\left\langle H_{\alpha, j}^{(\tau)}\right\rangle_{\tau, \alpha}^{(1,1)} & \left\langle H_{\alpha, j}^{(\tau)} \sigma_{2}\right\rangle_{\tau, \alpha}^{(1,1)} & \left\langle H_{\alpha, j}^{(\tau)}\right\rangle_{\tau, \alpha}^{(1,2)} & \left\langle H_{\alpha, j}^{(\tau)} \sigma_{2}\right\rangle_{\tau, \alpha}^{(1,2)} & \ldots \\
\left\langle\sigma_{2} H_{\alpha, j}^{(\tau)}\right\rangle_{\tau, \alpha}^{(1,1)} & \left\langle\sigma_{2} H_{\alpha, j}^{(\tau)} \sigma_{2}\right\rangle_{\tau, \alpha}^{(1,1)} & \left\langle\sigma_{2} H_{\alpha, j}^{(\tau)}\right\rangle_{\tau, \alpha}^{(1,2)} & \left\langle\sigma_{2} H_{\alpha, j}^{(\tau)} \sigma_{2}\right\rangle_{\tau, \alpha}^{(1,2)} & \ldots \\
\left\langle H_{\alpha, j}^{(\tau)}\right\rangle_{\tau, \alpha}^{(2,1)} & \left\langle H_{\alpha, j}^{(\tau)} \sigma_{2}\right\rangle_{\tau, \alpha}^{(2,1)} & \left\langle H_{\alpha, j}^{(\tau)}\right\rangle_{\tau, \alpha}^{(2,2)} & \left\langle H_{\alpha, j}^{(\tau)} \sigma_{2}\right\rangle_{\tau, \alpha}^{(2,2)} & \ldots \\
\left\langle\sigma_{2} H_{\alpha, j}^{(\tau)}\right\rangle_{\tau, \alpha}^{(2,1)} & \left\langle\sigma_{2} H_{\alpha, j}^{(\tau)} \sigma_{2}\right\rangle_{\tau, \alpha}^{(2,1)} & \left\langle\sigma_{2} H_{\alpha, j}^{(\tau)}\right\rangle_{\tau, \alpha}^{(2,2)} & \left\langle\sigma_{2} H_{\alpha, j}^{(\tau)} \sigma_{2}\right\rangle_{\tau, \alpha}^{(2,2)} & \ldots \\
\vdots & \vdots & \vdots & \vdots & \ddots
\end{array}\right)
$$

Here $\mathbf{h} \ldots \mathbf{i}_{\tau, \alpha}^{\left(n, n^{\prime}\right)} \equiv \mathbf{h} \phi_{\alpha}^{(\tau, n)}|\ldots| \phi_{\alpha}^{\left(\tau, n^{\prime}\right)} \mathbf{i}$ denotes a matrix element between positiveenergy eigenstates of the radial Schrödinger equation. By construction, we have

$$
\mathrm{H}_{\alpha, 0}^{(\tau)}=\left(\begin{array}{ccccc}
E_{\alpha, 0}^{(\tau, 1)} & 0 & 0 & 0 & \vdots \\
0 & -E_{\alpha, 0}^{(\tau, 1)} & 0 & 0 & \vdots \\
0 & 0 & E_{\alpha, 0}^{(\tau, 2)} & 0 & \vdots \\
0 & 0 & 0 & -E_{\alpha, 0}^{(\tau, 2)} & \vdots \\
\vdots & \vdots & \vdots & \vdots & \ddots
\end{array}\right)
$$

The explicit form of $\mathbf{H}_{\alpha, 1}^{(\tau)}$ depends, in principle, on the details of the confinement. However, as shown below, we can use general properties of the basis states to gain some insight about its matrix elements.

For the SQW case, modifications are easily made by not using the energyreflection symmetry $\left(\left|\phi_{\alpha}^{(\tau,-n)} \mathbf{i}=\sigma_{2}\right| \phi_{\alpha}^{(\tau, n)} \mathbf{i}\right)$ and performing the general form 
of a change in basis.

Furthermore, once the form of $\mathrm{H}_{\alpha, 1}^{(\tau)}$ is known, we can use perturbation theory approximations to find the $n^{\text {th }}$ subband-dispersion relation. This is done by just solving the $(n,-n) 2 \times 2$ block-diagonal Hamiltonians where we ignore the interactions between $(n, \pm m)$ subbands where $n \sigma m$. Generally, this is a valid approach since the largest magnitudes in $\mathrm{H}_{\alpha, 0}^{(\tau)}$ are the diagonal energy terms (by construction, also the only terms) and in $\mathrm{H}_{\alpha, 1}^{(\tau)}$ are the $(n,-n)$ offdiagonal terms 4 . Naturally, the coupling between different subbands will also decrease depending on the energy difference between the subbands.

A pictorial image of the magnitudes of $\mathrm{H}_{\alpha, 1}^{(\tau)}$ elements, based off the SLG case [as presented in Sec. (3.2)], can be seen in the following equation

$\mathrm{H}_{\alpha, 1}^{(\tau)}=$

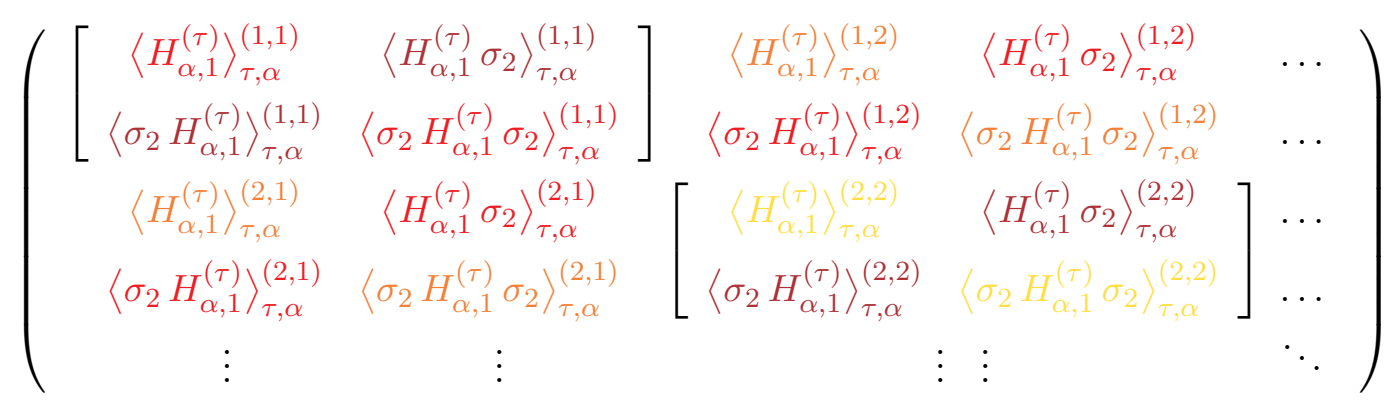

where the darkness of the colour indicates the magnitude of the element (darker $\equiv$ larger: maroon $>$ red $>$ orange $>$ yellow). The $(n,-n) 2 \times 2$ blockdiagonal Hamiltonians $\mathrm{H}_{\alpha, 1}^{(\tau, n)}$ are indicated by the square brackets.

We see that, although the off-diagonal $(n,-n)$ subband terms are consistently the largest (maroon) for all $n$, the magnitude of coupling terms between $(n, \pm m)$ subbands can become larger than diagonal terms. This is usually compensated by the large energy terms in the diagonal entries of $\mathrm{H}_{\alpha, 0}^{(\tau)}$ and a cancelling of coupling effect:5. However, for higher $n$ subbands the $(n, n \pm 1)$ subband coupling terms may have a non-negligible effect due to the decrease in magnitude of the $(n, n)$ diagonal terms. This could result in a slight shift of the subband energies that is not taken account of. We assume

\footnotetext{
${ }^{4}$ This will become more apparent in the following chapters.

${ }^{5}$ Subbands have a tendency to repel each other - this would lead to a certain amount of the $n \pm 1$ coupling effects to cancel.
} 
that this generally has no crucial effect on the actual physics of the subbands, although we only completely trust the results from the lowest subbands.

Thus, the general $2 \times 2$ block-diagonal Hamiltonian in the new basis is given by

$$
\mathbf{H}_{\alpha}^{(\tau, n)}=\left(\begin{array}{cc}
E_{\alpha, 0}^{(\tau, n)}+\left\langle H_{\alpha, 1}^{(\tau)}\right\rangle_{\tau, \alpha}^{(n, n)} & \left\langle H_{\alpha, 1}^{(\tau)} \sigma_{2}\right\rangle_{\tau, \alpha}^{(n, n)} \\
\left\langle\sigma_{2} H_{\alpha, 1}^{(\tau)}\right\rangle_{\tau, \alpha}^{(n, n)} & -E_{\alpha, 0}^{(\tau, n)}+\left\langle\sigma_{2} H_{\alpha, 1}^{(\tau)} \sigma_{2}\right\rangle_{\tau, \alpha}^{(n, n)}
\end{array}\right)
$$

where the $\pm n^{\text {th }}$ subband energy dispersion is given by

$$
\begin{aligned}
E_{\alpha}^{(\tau, \pm n)}= & \frac{\left\langle H_{\alpha, 1}^{(\tau)}\right\rangle_{\tau, \alpha}^{(n, n)}+\left\langle\sigma_{2} H_{\alpha, 1}^{(\tau)} \sigma_{2}\right\rangle_{\tau, \alpha}^{(n, n)}}{2} \\
& \pm \sqrt{\left[E_{\alpha, 0}^{(\tau, n)}+\frac{\left\langle H_{\alpha, 1}^{(\tau)}\right\rangle_{\tau, \alpha}^{(n, n)}-\left\langle\sigma_{2} H_{\alpha, 1}^{(\tau)} \sigma_{2}\right\rangle_{\tau, \alpha}^{(n, n)}}{2}\right]^{2}+\left|\left\langle H_{\alpha, 1}^{(\tau)} \sigma_{2}\right\rangle_{\tau, \alpha}^{(n, n)}\right|^{2}}
\end{aligned}
$$

Now that we have presented the overall procedure to determine subbanddispersion relations, we move onto understanding the changes due to the effects of an added magnetic field.

\subsection{Ring-enclosed magnetic (Aharonov-Bohm) flux}

For our system, we consider a magnetic field, $\mathbf{B}$, that pierces the centre of the ring plane. In the presence of a magnetic field $\mathbf{B}=\nabla \times \mathbf{A}$ perpendicular to the ring plane, the wave vector $\mathbf{k}$ entering Eqns. (2.1), 2.4) and (2.7) for the bulk SLG, TMD and SQW systems is substituted according to the familiar rule 25, 26

$$
\mathbf{k} \rightarrow \mathbf{k}+\frac{e}{\hbar} \mathbf{A}
$$

where we use the convention for charge $q \rightarrow-e$ ( $e$ being absolute value of the charge of an electron). Using polar coordinates and the vector potential 
$\mathbf{A}=[\phi /(2 \pi r)] \hat{\varphi}$ for an infinitesimally thin tube of magnetic flux $\phi$ piercing the ring plane at its origin, one finds the usual substitutions $24,35,36$

$$
k_{\varphi} \rightarrow k_{\varphi}+\frac{\phi}{\phi_{0} r} \quad \Leftrightarrow \quad l \rightarrow l(\phi)=l+\frac{\phi}{\phi_{0}},
$$

where $\phi_{0} \equiv 2 \pi \hbar / e$ is the magnetic flux quantum and no change in $k_{r}$. Replacing $l \rightarrow l(\phi)$ in Eqns. 2.1), 2.4) and (2.7), respectively, yields the SLG, TMD and SQW ring dispersions as a function of the magnetic flux penetrating the ring area.

We have now found that only the operator $k_{\varphi}$ and angular quantum number $l$ change in the presence of a magnetic field. Hence, we have all the relevant tools to tackle transport properties of ring system, which is explored in the next section.

\subsection{Ring confinement with leads}

For electronic transport through 2D material quantum rings, we consider a ring system that is attached to leads with a magnetic flux, $\phi$, that pierces the centre of the ring, as was described in Sec. 2.3.). This generic scenario is sketched schematically in Fig. (2.1) where all the relevant scattering amplitudes are indicated.

The linear electric conductance $G$ is related to the transmission probability between scattering states from the two leads via 57

$$
G=\frac{e^{2}}{2 \pi \hbar} \sum_{\nu_{1}, \nu_{2}} T_{\nu_{2} \nu_{1}}(E)
$$

where $T_{\nu_{2} \nu_{1}}$ is the transmission function with the labels $\nu_{1}\left(\nu_{2}\right)$ for states at fixed energy $E$ in lead 1 (2) including all possible (i.e., orbital as well as realspin and valley) degrees of freedom for the charge carriers. To determine the transmission functions $T_{\nu_{2} \nu_{1}}(E)$, the scattering problem for electronic probability waves has to be solved for the ring structure as was done, e.g., in Refs. 37], 36] and 35.

Such a calculation is based on the description of each junction by a threeterminal scattering matrix that links lead states at a fixed energy to ring 


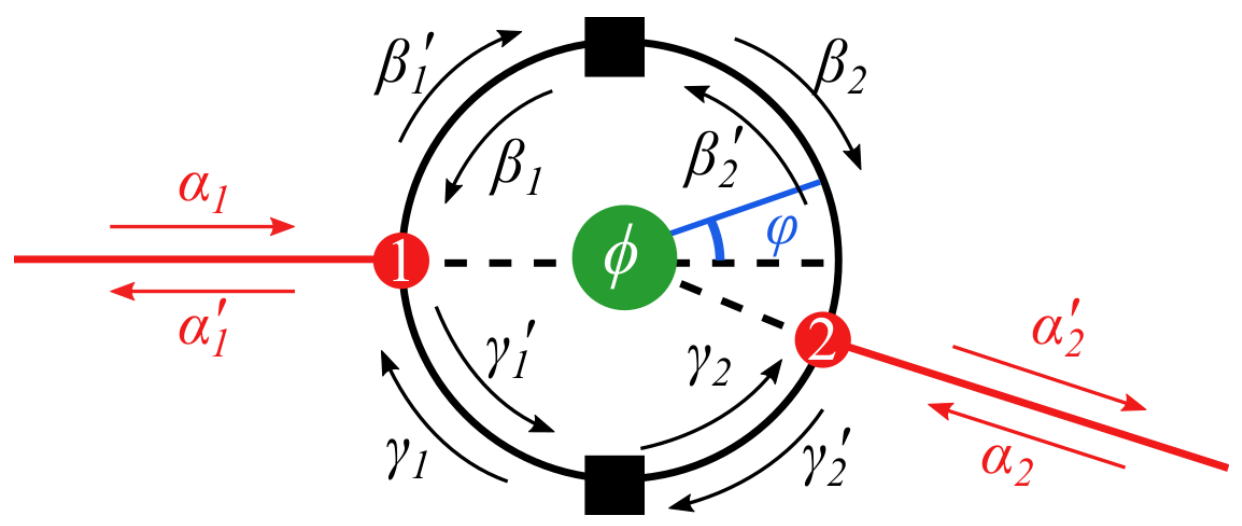

Figure 2.1: Electronic transport through a quantum ring conductor with a magnetic flux, $\phi$, through the centre. Leads are connected at two junctions labelled 1 and 2 where charge carriers are distributed between (incoming and outgoing) scattering states in the leads and (right-moving and left-moving) ring eigenstates. $\alpha, \beta$ and $\gamma$ are scattering amplitudes. The squares represent the points where we match scattering amplitudes from the right and left - this is necessary due to the non-trivial phase change of the eigenstates. Figure is adapted from Ref. [35].

eigenstates at that same energy. Here we adopt the general form of a Tjunction scattering matrix 35, 37], $\mathbf{S}$, which is based on a model that is consistent with basic symmetries and conservation laws. $\mathbf{S}$ is applied to the three incoming waves $\mathbf{a}_{i} \equiv\left(\alpha_{i}, \beta_{i}, \gamma_{i}\right)$ at the T-junction (labelled $i=\{1,2\}$ ) to describe the three outgoing waves $\mathbf{a}_{i}^{\prime} \equiv\left(\alpha_{i}^{\prime}, \beta_{i}^{\prime}, \gamma_{i}^{\prime}\right)$ where $\alpha$ is in the current lead and $\beta \& \gamma$ are in the ring branches [see Fig. (2.1)]. This gives the relation

$$
\mathbf{a}_{i}^{\prime}=\mathbf{S} \mathbf{a}_{i}
$$

We assume $\mathbf{S}$ is real and symmetric with respect to two branches (i.e. incoming electrons from the lead are scattered evenly into both branches of the ring). Thus $\mathbf{S}$ is given by

$$
\mathbf{S}=\left(\begin{array}{ccc}
-(a+b) & \epsilon_{c}^{1 / 2} & \epsilon_{c}^{1 / 2} \\
\epsilon_{c}^{1 / 2} & a & b \\
\epsilon_{c}^{1 / 2} & b & a
\end{array}\right)
$$

where $\epsilon_{c}$ determines the the coupling strength between the lead and the ring 
and $a$ and $b$ can be found using the probability current conservation rule of unitarity to give

$$
\begin{gathered}
(a+b)^{2}+2 \epsilon_{c}=1 \\
a^{2}+b^{2}+\epsilon_{c}=1 .
\end{gathered}
$$

Rearranging the equations, we find that $a$ and $b$ can be given in terms of $\epsilon_{c}$ :

$$
\begin{aligned}
& a_{ \pm}= \pm \frac{1}{2}\left(\sqrt{ } \overline{1-2 \epsilon_{c}}-1\right) \\
& b_{ \pm}= \pm \frac{1}{2}\left(\sqrt{ } \overline{1-2 \epsilon_{c}}+1\right)
\end{aligned}
$$

where the solutions $\left\{a_{+}, b_{+}\right\}$and $\left\{a_{-}, b_{-}\right\}$correspond to the same physical description of the system. Without loss of generality, we use solutions $\left\{a_{+}, b_{+}\right\}$henceforth. We have also neglected two other sets of solutions that are not of physical interest to us - refer to Ref. [35] for more information.

There are obvious bounds for $\epsilon_{c}$ : $0 \leq \epsilon_{c} \leq \frac{1}{2}$. $\epsilon_{c}=1 / 2$ corresponds to a completely transparent junction since $a+b=0$ in this case. In the other extreme, $\epsilon_{c}=0$ corresponds to all electrons in the current lead being reflected back since $a+b=1$. In this case $b^{2}=1$ which means the electrons in the ring do not notice the presence of a junction when they travel from one half to the other.

To find the transmission of the ring, we assume a single incoming current that enters via junction 1 (thus $\left|\alpha_{1}\right|^{2}=1$ ) which means the transmission is given by $T=\left|\alpha_{2}^{\prime}\right|^{2}$. To find $\alpha_{2}^{\prime}$, we match the wavefunctions at the squares in Fig. 2.1) which will result in

$$
\left(\begin{array}{l}
\beta_{2} \\
\beta_{2}^{\prime}
\end{array}\right)=e^{-i \theta_{1}}\left(\begin{array}{cc}
e^{i \phi_{\mathrm{s} 1}} & 0 \\
0 & e^{-i \phi_{\mathrm{s} 1}}
\end{array}\right)\left(\begin{array}{l}
\beta_{1}^{\prime} \\
\beta_{1}
\end{array}\right)
$$

and

$$
\left(\begin{array}{c}
\gamma_{1} \\
\gamma_{1}^{\prime}
\end{array}\right)=e^{-i \theta_{2}}\left(\begin{array}{cc}
e^{i \phi_{\mathrm{s} 2}} & 0 \\
0 & e^{-i \phi_{\mathrm{s} 2}}
\end{array}\right)\left(\begin{array}{l}
\gamma_{2}^{\prime} \\
\gamma_{2}
\end{array}\right)
$$

where $\phi_{\mathrm{s} 1 / 2}$ is a flux-independent dynamic phase change and $\theta_{1 / 2}$ is the geometric phase change. Both these phases arise from the change of phase 
factors attached to the wavefunctions as the charges move around the ring as was described in Sec. (1.4). The Aharanov-Bohm phase and the spin 1/2 phase both appear in $\theta_{1 / 2}$ while dynamic phases that are dependent on energy appear in $\phi_{\mathrm{s} 1 / 2}$. These also specifically depend on the geometry of the system such as the position of the junctions. Generally, the total dynamic phase, $\phi_{\mathrm{s}}$, and geometric phase, $2 \theta$, that enter the transmission are found to be

$$
\begin{gathered}
\phi_{\mathrm{s}}=\frac{\phi_{\mathrm{s} 1}+\phi_{\mathrm{s} 2}}{2} \\
\theta=\frac{\theta_{1}+\theta_{2}}{2} .
\end{gathered}
$$

Using Eqns. 2.30), 2.31, 2.36 and 2.37) we can rearrange to find $\alpha_{2}^{\prime}$ and thus the transmission. The details of this calculation can be found in Ref. 35. The transmission function for a perfectly symmetric ring where $\theta_{1}=\theta_{2}=\theta$ and $\phi_{\mathrm{s} 1}=\phi_{\mathrm{s} 2}=\phi_{\mathrm{s}}$ is

$$
T\left(\phi_{\mathrm{s}}, \theta, \epsilon_{c}\right)=\frac{4 \epsilon_{c}^{2} \sin ^{2} \phi_{\mathrm{s}} \cos ^{2} \theta}{\left[a^{2}+b^{2} \cos 2 \theta-\left(1-\epsilon_{c}\right) \cos 2 \phi_{\mathrm{s}}\right]^{2}+\epsilon_{c}^{2} \sin ^{2} 2 \phi_{\mathrm{s}}} .
$$

Note that in the presence of a magnetic flux $\phi$, only the geometric phase $\theta(\phi)$ becomes dependent on it, as expected.

In Chapter 5, we apply this theory to our model for the ring bandstructure and determine the transfer matrices that describe electron propagation through the two ring branches that are connected to the leads. In particular, we derive explicit expressions for the Berry phase, $\theta$, and $\phi_{\mathrm{s}}$ in terms of the injection energy and ring parameters. The conductance can then be derived via Eqn. (2.29) where we take in to account all spin and pseudo-spin degrees of freedom. To keep this discussion simple, we assume that only the ring subband with label $n$ contributes to transport.

The conductance of QR systems for our specific materials will be discussed in detail in Chapter 5 and their Berry phases will be explored in Chapter 6 . Having developed the general formalism for tackling confinement in QRs, we proceed to apply this to SLG, the simplest possible Dirac system, in the next chapter. 


\section{Chapter 3}

\section{Single-layer graphene}

In this chapter we explore the ring confinement properties, such as the dispersion relations, of single-layer graphene (SLG) ring structures using the methodology as described by Sec. (2.2).

First, we map $H_{\mathrm{SLG}, 0}^{(\tau)}$ into a $1 \mathrm{D}$ Dirac equation to which we apply a hard-wall confinement. After we determine the secular energy equation via straightforward calculation, we discover that using a particular parametrisation of the secular equation allows for the existence of a "universal" energy solution, i.e. the energies are completely determined by the confinement energy $\hbar v / W$ and the band gap $V_{0}$. We also find and investigate the topological properties of pure evanescent bound states, with energies less than $\left|V_{0}\right|$, for an inverted band structure $V_{0}<0$.

Then, a simple massless-Dirac case is explored for which we find the full angular dependence of the Hamiltonian and subband energy dispersions using realistic approximations. These are found to agree with previous work [5]. Finally a general mass-confinement case is explored for SLG and singlelayer transition-metal dichalcogenide (TMD) rings. We find the full subband energy-dispersion relation in terms of the confinement-energy and gap size. 


\subsection{Ring structure in SLG}

To solve the radial Schrödinger equation 2.18 with the SLG Hamiltonian (2.15a), we make the Ansatz

$$
\phi_{\mathrm{SLG}}^{(\tau, n)}(r)=\frac{\chi_{\text {SLG }}^{(\tau, n)}(r)}{2 \pi r}
$$

Notice that we do not have to worry about the transformation at $r=0$ due to the ring structure. Straightforward calculation, using Eqns. 2.9a and (2.10c, yields

$$
k_{r}\left(\frac{\chi_{\mathrm{SLG}}^{(\tau, n)}(r)}{\overline{2 \pi r}}\right) \equiv \frac{1}{\overline{2 \pi r}}\left(-i \partial_{r}\right) \chi_{\mathrm{SLG}}^{(\tau, n)}(r)
$$

Hence the radial Schrödinger equation for a SLG ring [obtained using Eqns. (2.15a) and 2.18] translates into a 1D Dirac equation with a widely studied mass confinement $48,58,62$ for the function $\chi_{\mathrm{SLG}}^{(\tau, n)}(r)$ :

$$
-i \frac{d \chi_{\mathrm{SLG}}^{(\tau, n)}}{d r} \tau \sigma_{1}+\frac{1}{\hbar v}\left[V(r) \sigma_{3}-E_{\mathrm{SLG}, 0}^{(\tau, n)} \sigma_{0}\right] \chi_{\mathrm{SLG}}^{(\tau, n)}=0
$$

to which we apply a hard-wall boundary that is given by

$$
V(r)=\left\{\begin{array}{ll}
V_{0} & \text { for } r_{\mathrm{i}}<r<r_{\mathrm{o}} \\
\infty & \text { elsewhere }
\end{array} .\right.
$$

where $r_{i}$ and $r_{0}$ are the inner and outer ring radii, respectively. We employ the following notation for the width $W$ and average radius $R$ :

$$
\begin{aligned}
W & =r_{\mathrm{o}}-r_{\mathrm{i}} \\
R & =\frac{r_{\mathrm{o}}+r_{\mathrm{i}}}{2}
\end{aligned}
$$

The eigenstates for energy $E \equiv E_{\mathrm{SLG}, 0}^{(\tau, n)}$ have the general form

$$
\chi_{\mathrm{SLG}}^{(\tau, n)}(r)=c_{1 \kappa}^{(\tau, n)}\left(\begin{array}{c}
1 \\
\tau \sigma \gamma
\end{array}\right) \mathrm{e}^{i \kappa r}+c_{2 \kappa}^{(\tau, n)}\left(\begin{array}{c}
1 \\
-\tau \sigma \gamma
\end{array}\right) \mathrm{e}^{-i \kappa r}
$$


where $\sigma \equiv \operatorname{sgn}\left(E+V_{0}\right), \operatorname{sgn}(\alpha) \equiv \alpha /^{\sqrt{ }} \overline{\alpha^{2}}$ and

$$
\begin{aligned}
\kappa & =\frac{1}{\hbar v} \sqrt{E^{2}-V_{0}^{2}}, \\
\gamma & =\sqrt{\frac{E-V_{0}}{E+V_{0}}} .
\end{aligned}
$$

These quantities are real for $|E|>\left|V_{0}\right|$. For $|E|<\left|V_{0}\right|$, we have $\kappa \equiv i \bar{\kappa}$ and $\gamma \equiv i \bar{\gamma}$ where $\bar{\kappa}$ and $\bar{\gamma}$ are now the real parts. They are given by

$$
\begin{aligned}
& \bar{\kappa}=\frac{1}{\hbar v} \sqrt{V_{0}^{2}-E^{2}}, \\
& \bar{\gamma}=\sqrt{\frac{V_{0}-E}{V_{0}+E}} .
\end{aligned}
$$

Now, we have the general form of the wavefunction without having imposed boundary conditions yet. In the next section, we will determine the eigenenergies when hard-wall boundary conditions are applied.

\subsubsection{Universal energy curve}

The general method of setting the wavefunction to zero at the boundaries $\left(r_{i}\right.$ and $\left.r_{o}\right)$, i.e. $\chi_{\mathrm{SLG}}^{(\tau, n)}\left(r_{i}\right)=0=\chi_{\mathrm{SLG}}^{(\tau, n)}\left(r_{o}\right)$, does not result in a normalisable result in the case of a 1D Dirac equation. This is because the system is overdetermined and thus would only possess the trivial solution. To overcome this issue, we impose the zero-current boundary conditions [5,48,63 65] which is equivalent to a hard-wall confinement in this system. The conditions are thus given by

$$
\begin{aligned}
\chi_{\mathrm{SLG}}^{(\tau, n)}\left(r_{\mathrm{i}}\right) & =-\tau \sigma_{2} \chi_{\mathrm{SLG}}^{(\tau, n)}\left(r_{\mathrm{i}}\right), \\
\chi_{\mathrm{SLG}}^{(\tau, n)}\left(r_{\mathrm{o}}\right) & =\tau \sigma_{2} \chi_{\mathrm{SLG}}^{(\tau, n)}\left(r_{\mathrm{o}}\right)
\end{aligned}
$$

at the inner $\left(r=r_{\mathrm{i}}\right)$ and outer $\left(r=r_{\mathrm{o}}\right)$ ring radii, respectively. This yields the secular equation

$$
\tan (\kappa W)=\sigma \frac{2 \gamma}{\gamma^{2}-1} \equiv-\frac{\hbar v}{V_{0}} \kappa
$$


The trivial solution $\kappa=0$ has $\gamma=0$ and thus yields the unphysical spinor $\chi_{\mathrm{SLG}}(r) \equiv(0,0)^{T}$. To make contact with previous works, we further examine the solutions of the secular Eqn. (3.10) using the addition theorem for tangents. It is possible to distinguish two subsets of solutions to Eqn. (3.10) which were derived by Ref. [58] to be

$$
\sigma \gamma=\left\{\begin{array}{r}
\cot \left(\frac{\kappa W}{2}\right) \\
-\tan \left(\frac{\kappa W}{2}\right)
\end{array}\right.
$$

where solutions to Eqn. (3.11a) will result in odd $n>0$ states and Eqn. (3.11b) will result in even $n>0$ states. Notice also that using the transformation $E_{\mathrm{SLG}, 0}^{(\tau, n)} \rightarrow-E_{\mathrm{SLG}, 0}^{(\tau, n)}$ in Eqn. 3.11a) [or Eqn. (3.11b] will result in Eqn. 3.11b [or Eqn. (3.11a] since $\gamma \rightarrow 1 / \gamma, \kappa \rightarrow \kappa$ and $\sigma \rightarrow-\sigma$. This exemplifies the high degree of symmetry in these solutions.

Using the parameter $\hbar v / W$, we can use the parametrisation: $E /(\hbar v / W)$ and $V_{0} /(\hbar v / W)$, such that the solutions to Eqns. 3.11a and (3.11b are "universal". This means that the width and Fermi velocity do not have to be exactly specified in order to have a solution - only the quantities $\hbar v / W$ and $V_{0}$ are necessary to determine the energies of the system. Hence $\hbar v / W$ is also known as the confinement energy. The parameterisation derivations can be seen as below:

$$
\operatorname{sgn}\left(E+V_{0}\right) \sqrt{\frac{\frac{E W}{\hbar v}-\frac{V_{0} W}{\hbar v}}{\frac{E W}{\hbar v}+\frac{V_{0} W}{\hbar v}}}=\left\{\begin{array}{c}
\cot \left(\frac{\sqrt{\left(\frac{E W}{\hbar v}\right)^{2}-\left(\frac{V_{0} W}{\hbar v}\right)^{2}}}{2}\right) \\
-\tan \left(\frac{\sqrt{\left(\frac{E W}{\hbar v}\right)^{2}-\left(\frac{V_{0} W}{\hbar v}\right)^{2}}}{2}\right)
\end{array}\right.
$$

where $E /(\hbar v / W)$ is now purely a function of $V_{0} /(\hbar v / W)$.

For negative values of $V_{0}$ where

$$
\frac{V_{0} W}{\hbar v} \leq-1
$$

there exist evanescent edge states within the $\pm\left|V_{0}\right|$ gap. This is because $\left(V_{0}, E\right)=(-1,1)$, which is a solution to Eqn. (3.12a), is the value below 
which the energy of the lowest-subband $(n=1)$ passes from above the gap to below. Below this limit, $\kappa$ and $\gamma$ both become purely imaginary, as given by Eqns. (3.8a and (3.8b), and hence result in an evanescent wavefunction. This is where SLG becomes topologically non-trivial thus allowing for the presence of evanescent TPES in the gap.

We depict the universal energy curves for the first three positive lowestsubbands in Fig. (3.1) 1). It can be seen that the lowest-subband traverses below the band gap $\left|V_{0}\right|$ as described by the limit in Eqn. (3.13). The figure shows the distinct difference between the behaviour of the lowest-subband when it becomes a TPES $[E /(\hbar v / W) \rightarrow 0$ as $V /(\hbar v / W) \rightarrow-\infty]$ compared to the higher subbands that obey the classical behaviour $[E /(\hbar v / W) \rightarrow \infty$ as $V /(\hbar v / W) \rightarrow-\infty]$.

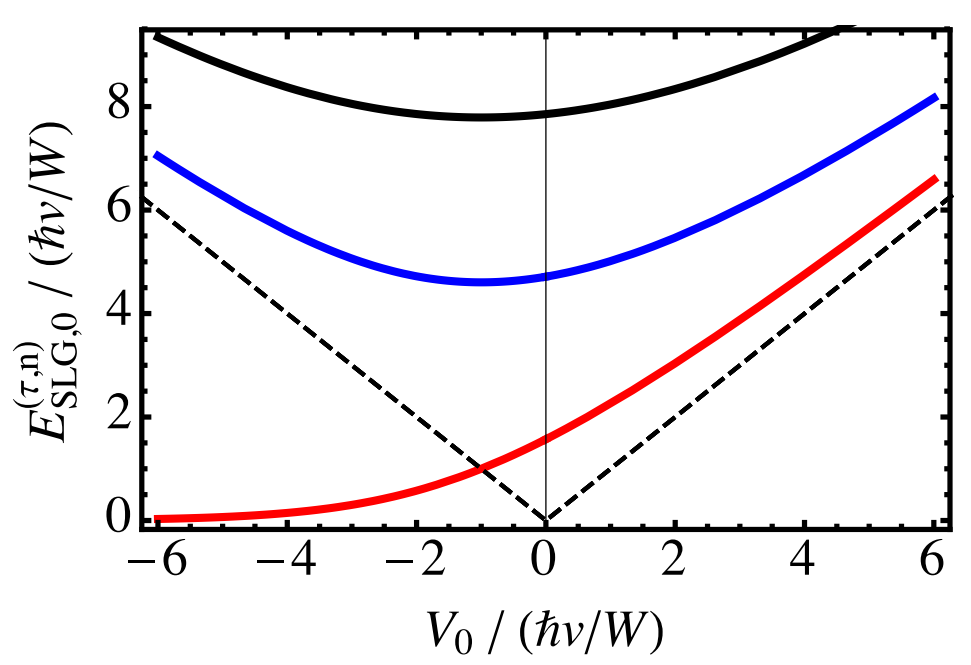

Figure 3.1: $\quad$ Three positive $n$ subband energy curves as a function of the universal parameter $V_{0} /(\hbar v / W)$ for $S L G$ rings. The universal energy curve for $n=1$ (red), 2 (blue) and 3 (black) are shown for all relevant parameters. For reference, the black-dashed line represents where the gap is situated for a constant width, i.e. $E_{\mathrm{SLG}, 0}^{(\tau, n)}=\left|V_{0}\right|$. If the energy of a state is below this line then it is evanescent and a topological bound state.

Having found the universal energy curves, we now proceed to find the eigenstates of the $H_{\mathrm{SLG}, 0}^{(\tau)}$ system in the next section.

\footnotetext{
${ }^{1}$ Recall that since $H_{\mathrm{SLG}, 0}^{(\tau)}$ is energy-reflection symmetric, $E_{\mathrm{SLG}, 0}^{(\tau, n)}=-E_{\mathrm{SLG}, 0}^{(\tau, n)}$ and thus we only have to consider positive energies.
} 


\subsubsection{Wavefunctions of SLG}

Using the boundary conditions, given by Eqns. (3.9a) and $3.9 \mathrm{~b}$, we find a relation between coefficients in the Ansatz [Eqn. (3.6)],

$$
c_{2 \kappa}^{(\tau, n)}=-\mathrm{e}^{2 i\left(\kappa r_{\mathrm{i}}-\arctan (\sigma \gamma)\right)} c_{1 \kappa}^{(\tau, n)}
$$

which allows us to find the eigenstates of the system. Straightforward calculation yields eigenstates of the form

$$
\chi_{\mathrm{SLG}}^{(\tau, n)}(r)=\mathbf{N}_{n}\left(\begin{array}{c}
\cos \left[\kappa_{n}(r-R)+\chi_{n}\right] \\
\tau \sigma \gamma_{n} i \sin \left[\kappa_{n}(r-R)+\chi_{n}\right]
\end{array}\right)
$$

for $r_{\mathrm{i}} \leq r \leq r_{\mathrm{o}}$ and $\chi_{\mathrm{SLG}}^{(\tau, n)}(r) \equiv 0$ elsewhere. Here $\kappa_{n}$ and $\gamma_{n}$ are the values of $\kappa$ and $\gamma$ corresponding to the $n^{\text {th }}$ quantised energy level. The phases $\chi_{n}$ and normalisation factors $\mathbf{N}_{n}$ are given by

$$
\begin{aligned}
\chi_{n} & =\frac{\kappa_{n} W}{2}-\frac{\pi}{2}+\arctan \left(\sigma \gamma_{n}\right) \\
\mathbf{N}_{n} & =\forall^{1} \overline{\bar{W}}\left[\frac{E_{n}\left(E_{n}+V_{0}\right)}{E_{n}^{2}+\frac{\hbar v}{W} V_{0}}\right]^{\frac{1}{2}} .
\end{aligned}
$$

where $E_{n}$ is the energy of the $n^{\text {th }}$ subband. The separation of the secular equation into Eqns. 3.11a) and 3.11b yields sets of momenta $\kappa_{n_{\mathrm{a}}}$ and $\kappa_{n_{\mathrm{b}}}$, respectively, for which the phases are $\chi_{n_{\mathrm{a}}}=0$ and $\chi_{n_{\mathrm{b}}}=-\pi / 2$. Hence, the corresponding eigenstates are those for which the conduction-band wave function is parity-even and parity-odd, respectively:

$$
\begin{aligned}
& \chi_{\mathrm{SLG}}^{\left(\tau, n_{\mathrm{a}}\right)}(r)=\mathbf{N}_{n_{a}}\left(\begin{array}{c}
\cos \left[\kappa_{n_{\mathrm{a}}}(r-R)\right] \\
\tau \sigma \gamma_{n_{\mathrm{a}}} i \sin \left[\kappa_{n_{\mathrm{a}}}(r-R)\right]
\end{array}\right), \\
& \chi_{\mathrm{SLG}}^{\left(\tau, n_{\mathrm{b}}\right)}(r)=\mathbf{N}_{n_{b}}\left(\begin{array}{c}
\sin \left[\kappa_{n_{\mathrm{b}}}(r-R)\right] \\
-\tau \sigma \gamma_{n_{\mathrm{b}}} i \cos \left[\kappa_{n_{\mathrm{b}}}(r-R)\right]
\end{array}\right) .
\end{aligned}
$$

One may wish to note that $\chi_{\mathrm{SLG}}^{(\tau, n)}(r)=\tau \sigma \sigma_{2} \chi_{\mathrm{SLG}}^{(\tau,-n)}(r)$. Thus the states are energy-reflection symmetric. The complete eigenstate $\mid \phi_{\mathrm{SLG}}^{(\tau, n)} \mathbf{i}$ is given by our Ansatz in Eqn. (3.1). 
Now that we have developed a full description of the $H_{\mathrm{SLG}, 0}^{(\tau)}$ system, we move on to transforming the $H_{\mathrm{SLG}, 1}^{(\tau)}$ into the basis of the eigenstates of $H_{\mathrm{SLG}, 0}^{(\tau)}$ and finding the general subband energy dispersion. In the next section, we do this for a specific example: the massless-Dirac case.

\subsection{Massless-Dirac case}

A perfect SLG QR is represented by the massless-Dirac case where $V_{0}=0$, i.e., $\gamma=1$. Thus, SLG rings are generally confinement dominated since the confinement energy $\hbar v / W$ is much larger than the gap size $V_{0}=0$. In this case, the secular Eqn. 3.10 has the following physical solutions:

$$
\begin{aligned}
\kappa_{n} & =\left(n-\frac{1}{2}\right) \frac{\pi}{W}, \\
E_{\mathrm{SLG}, 0}^{(\tau, n)} & =\left(n-\frac{1}{2}\right) \frac{\pi \hbar v}{W},
\end{aligned}
$$

with positive integer $n$. The result in Eqn. 3.17b recovers the expression for SLG-ring subband edges given before in Ref. [5]. Specialisation of Eqns. $(3.15$ a-c) to the present situation yields the eigenspinors

$$
\chi_{\mathrm{SLG}}^{(\tau, n)}(r)=\forall^{1} \overline{\bar{W}}\left(\begin{array}{c}
\cos \left[\left(n-\frac{1}{2}\right) \pi \frac{r-R}{W}+(n-1) \frac{\pi}{2}\right] \\
\tau i \sin \left[\left(n-\frac{1}{2}\right) \pi \frac{r-R}{W}+(n-1) \frac{\pi}{2}\right]
\end{array}\right) .
$$

where the normalisation factor becomes $1 / \sqrt{ } \bar{W}$. Distinguishing the cases of odd $[n=2 \nu-1$ which corresponds to secular Eqn. (3.11a) $]$ and even $[n=2 \nu$ which corresponds to secular Eqn. [3.11b] ] main quantum numbers, we can write

$$
\begin{aligned}
\chi_{\mathrm{SLG}}^{(\tau, 2 \nu-1)}(r) & =\sqrt{ } \frac{1}{\bar{W}}\left(\begin{array}{c}
\cos \left[\left(2 \nu-\frac{3}{2}\right) \pi \frac{r-R}{W}\right] \\
\tau i \sin \left[\left(2 \nu-\frac{3}{2}\right) \pi \frac{r-R}{W}\right]
\end{array}\right), \\
\chi_{\mathrm{SLG}}^{(\tau, 2 \nu)}(r) & =\sqrt{\frac{1}{W}}\left(\begin{array}{c}
\sin \left[\left(2 \nu-\frac{1}{2}\right) \pi \frac{r-R}{W}\right] \\
-\tau i \cos \left[\left(2 \nu-\frac{1}{2}\right) \pi \frac{r-R}{W}\right]
\end{array}\right) .
\end{aligned}
$$


Note that the total particle density for all states is uniform/nodeless across the ring width (i.e. $\chi_{\mathrm{SLG}}^{(\tau, n)}\left(r_{i / o}\right) \sigma 0$ ), in contrast to the nonrelativistic case.

It is straightforward to see the existence of symmetries in the components of the $\mathrm{H}_{\mathrm{SLG}, 1}^{(\tau)}$ matrix. We find that

$$
\mathrm{h} H_{\mathrm{SLG}, 1}^{(\tau)} \mathbf{i}_{\tau, \mathrm{SLG}}^{\left(n, n^{\prime}\right)}=\mathrm{h}_{2} H_{\mathrm{SLG}, 1}^{(\tau)} \sigma_{2} \mathbf{i}_{\tau, \mathrm{SLG}}^{\left(n, n^{\prime}\right)}
$$

and

$$
\mathrm{h}_{2} H_{\mathrm{SLG}, 1}^{(\tau)} \mathbf{i}_{\tau, \mathrm{SLG}}^{\left(n, n^{\prime}\right)}=\mathrm{h} H_{\mathrm{SLG}, 1}^{(\tau)} \sigma_{2} \mathbf{i}_{\tau, \mathrm{SLG}}^{\left(n, n^{\prime}\right)}
$$

which hold due to the relation $\sigma_{2}^{2}=\sigma_{0}$. Noting that the size-quantisation energy scale for the radial equation is $\hbar v / W$ [see Eqn. (3.17b)], we measure the matrix elements of $\mathrm{H}_{\alpha, 1}^{(\tau)}$ in the same unit. We also define the aspect ratio $\epsilon=W / R$ which will be important for future use. Using the wavefunctions of $H_{\mathrm{SLG}, 0}^{(\tau)}$, we can now determine the new form of $\mathrm{H}_{\alpha, 1}^{(\tau)}$ in the basis of $\mid \phi_{\mathrm{SLG}}^{(\tau, n)} \mathbf{i}$ and $\mid \phi_{\mathrm{SLG}}^{(\tau,-n)} \mathbf{i}$, as was set out in Chapter 2. It can be shown that using our Ansatz and Eqn. (3.18) we obtain:

$$
\begin{aligned}
& \mathrm{h} H_{\mathrm{SLG}, 1}^{(\tau)} \mathrm{i}_{\tau, \mathrm{SLG}}^{\left(n, n^{\prime}\right)}=\frac{\hbar v l}{W} \int_{r_{i}}^{r_{o}} \frac{1}{r}\left(\begin{array}{c}
\cos \left(\left(n-\frac{1}{2}\right) \pi \frac{r-r_{i}}{W}-\frac{\pi}{4}\right) \\
\tau i \sin \left(\left(n-\frac{1}{2}\right) \pi \frac{r-r_{i}}{W}-\frac{\pi}{4}\right)
\end{array}\right)^{\dagger} \sigma_{2} \\
&\left(\begin{array}{c}
\cos \left(\left(n^{\prime}-\frac{1}{2}\right) \pi \frac{r-r_{i}}{W}-\frac{\pi}{4}\right) \\
\tau i \sin \left(\left(n^{\prime}-\frac{1}{2}\right) \pi \frac{r-r_{i}}{W}-\frac{\pi}{4}\right)
\end{array}\right) \mathrm{d} r \\
&=\frac{\hbar v l \tau}{W} \int_{r_{i}}^{r_{o}} \frac{\sin \left(\left(n+n^{\prime}-1\right) \pi \frac{r-r_{i}}{W}-\frac{\pi}{2}\right)}{r} \mathrm{~d} r \\
&=-\frac{\hbar v l \tau}{W} \int_{r_{i}}^{r_{o}} \frac{\cos \left(\left(n+n^{\prime}-1\right) \pi \frac{r-r_{i}}{W}\right)}{r} \mathrm{~d} r
\end{aligned}
$$

and

$$
\begin{aligned}
& \mathrm{h}_{\mathrm{SLG}, 1}^{(\tau)} \sigma_{2} \mathrm{i}_{\tau, \mathrm{SLG}}^{\left(n, n^{\prime}\right)}= \frac{\hbar v l}{W} \int_{r_{i}}^{r_{o}} \frac{1}{r}\left(\begin{array}{c}
\cos \left(\left(n-\frac{1}{2}\right) \pi \frac{r-r_{i}}{W}-\frac{\pi}{4}\right) \\
\tau i \sin \left(\left(n-\frac{1}{2}\right) \pi \frac{r-r_{i}}{W}-\frac{\pi}{4}\right)
\end{array}\right)^{\dagger} \\
&\left(\begin{array}{c}
\cos \left(\left(n^{\prime}-\frac{1}{2}\right) \pi \frac{r-r_{i}}{W}-\frac{\pi}{4}\right) \\
\tau i \sin \left(\left(n^{\prime}-\frac{1}{2}\right) \pi \frac{r-r_{i}}{W}-\frac{\pi}{4}\right)
\end{array}\right) \mathrm{d} r \\
&=\frac{\hbar v l}{W} \int_{r_{i}}^{r_{o}} \frac{\cos \left(\left(n-n^{\prime}\right) \pi \frac{r-r_{i}}{W}\right)}{r} \mathrm{~d} r
\end{aligned}
$$


To write this in a more compact form we have

$$
\mathrm{h} H_{\mathrm{SLG}, 1}^{(\tau)} \mathbf{i}_{\tau, \mathrm{SLG}}^{\left(n, n^{\prime}\right)}=\frac{\hbar v}{W}(-\tau) l \mathrm{~F}\left(n+n^{\prime}-1, \epsilon\right)
$$

and

$$
\mathrm{h} H_{\mathrm{SLG}, 1}^{(\tau)} \sigma_{2} \mathbf{i}_{\tau, \mathrm{SLG}}^{\left(n, n^{\prime}\right)}=\frac{\hbar v}{W} l \mathbf{F}\left(n-n^{\prime}, \epsilon\right)
$$

The function $F$ is given by

$$
\mathbf{F}(p, \epsilon)=\frac{\epsilon}{\pi} \int_{-\frac{\pi}{2}}^{\frac{\pi}{2}} \frac{\mathrm{d} \rho}{1+\frac{\epsilon}{\pi} \rho} \cos \left(p\left[\rho+\frac{\pi}{2}\right]\right)
$$

where $p$ is an integer placeholder for $n+n^{\prime}-1$ or $n-n^{\prime}$ to give the relevant solutions. The solution to Eqn. (3.24) is given by

$$
\mathbf{F}(p, \epsilon)=\left(\operatorname{Ci}\left(\beta_{+}\right)-\operatorname{Ci}\left(-\beta_{-}\right)\right) \cos \left(\beta_{-}\right)-\left(\operatorname{Si}\left(\beta_{-}\right)+\operatorname{Si}\left(\beta_{+}\right)\right) \sin \left(\beta_{-}\right)
$$

where $\operatorname{Ci}(x)$ and $\operatorname{Si}(x)$ are the cosine and sine integral functions, respectively, and

$$
\beta_{ \pm}=p \pi\left(\frac{1}{2} \pm \frac{1}{\epsilon}\right)
$$

There is naturally a hierarchy in the magnitudes of the components of $\mathbf{H}_{\alpha, 1}^{(\tau)}$. As $p$ is increased incrementally, $\beta_{ \pm}$also increases which leads to a decrease in the size of the integral shown by Eqn. (3.24). Hence, the value of $p$ $\left(=n+n^{\prime}-1\right.$ or $\left.n-n^{\prime}\right)$ determines the magnitude of the components. This is exactly how the sizing of the matrix elements were found in Eqn. (2.24).

For $p \boldsymbol{6} 0$, the series expansion of $\mathbf{F}(p, \epsilon)$ in $\epsilon$ around $\epsilon=0$ gives

$$
\mathbf{F}(p, \epsilon)=\frac{2}{p^{2} \pi^{2}} \epsilon^{2}+\frac{3\left(\pi^{2} p^{2}-8\right)}{2 \pi^{4} p^{4}} \epsilon^{4}+\mathbf{O}\left(\epsilon^{6}\right)
$$

for odd $p$ and

$$
\mathbf{F}(p, \epsilon)=\frac{2}{p^{2} \pi^{2}} \epsilon^{3}+\frac{\left(\pi^{2} p^{2}-24\right)}{\pi^{4} p^{4}} \epsilon^{5}+\mathbf{O}\left(\epsilon^{7}\right)
$$

for even $p$. In the special case $p=0$ [i.e. $n=n^{\prime}$ for Eqn. 3.23 ] $\mathbf{F}(0, \epsilon)$ can 
be expressed in closed form as

$$
\mathrm{h} H_{\mathrm{SLG}, 1}^{(\tau)} \sigma_{2} \mathbf{i}_{\tau, \mathrm{SLG}}^{(n, n)}=\frac{\hbar v}{W} l \ln \left(\frac{1+\frac{\epsilon}{2}}{1-\frac{\epsilon}{2}}\right)
$$

In the limit of $\epsilon \rightarrow 0$, i.e., for a ring with large radius and small width, the leading contributions to the Hamiltonian are the terms proportional to $\mathbf{F}(0, \epsilon) \approx \epsilon+\mathrm{O}\left(\epsilon^{3}\right)$.

Keeping only the largest order of $\epsilon$ terms, the Hamiltonian $\mathbf{H}_{\alpha, 1}^{(\tau)}$ becomes a block-diagonal matrix with only the matrix elements $\mathrm{h} H_{\mathrm{SLG}, 1}^{(\tau)} \sigma_{2} \mathbf{i}_{\tau, \mathrm{SLG}}^{(n, n)}$ and $\mathrm{h}_{2} H_{\mathrm{SLG}, 1}^{(\tau)} \mathrm{i}_{\tau, \mathrm{SLG}}^{(n, n)}$ taken to be non-zero. Some previous works 66 68 have essentially assumed such a form of the Hamiltonian for the ideal-SLG (Dirac) ring limit.

However, a more accurate solution to the eigenvalue problem, given by Eqn. (2.21), is equivalent to solving

$$
\frac{\pi \hbar v}{W}\left(\begin{array}{cc}
n-\frac{1}{2} & \frac{W}{\pi R} l \\
\frac{W}{\pi R} l & -\left(n-\frac{1}{2}\right)
\end{array}\right)\left(\begin{array}{l}
a_{l, \tau, \mathrm{SLG}}^{( \pm n, n)} \\
b_{l, \tau, \mathrm{SLG}}^{( \pm n, n)}
\end{array}\right)=E_{\mathrm{SLG}}^{(\tau, n)}(l)\left(\begin{array}{c}
a_{l, \tau, \mathrm{SLG}}^{( \pm n, n)} \\
b_{l, \tau, \mathrm{SLG}}^{( \pm n, n)}
\end{array}\right)
$$

where the radial-size-quantisation energy $E_{\mathrm{SLG}, 0}^{(\tau, n)}$ enters as a mass term in the diagonal entries. Straightforward diagonalisation yields

$$
E_{\mathrm{SLG}}^{(\tau, \pm n)}(l)= \pm \frac{\hbar v}{W} \sqrt{\pi^{2}\left(n-\frac{1}{2}\right)^{2}+\left(\frac{W}{R}\right)^{2} l^{2}}
$$

Closer inspection of Eqn. (3.31) reveals that the leading correction to the energies is of order $\epsilon^{2}$, which is of the same order as the diagonal contribution $\mathrm{h} H_{\mathrm{SLG}, 1}^{(\tau)} \mathrm{i}_{\tau, \mathrm{SLG}}^{(n, n)}$. As established in Chapter 2 and Eqn. 2.25 , the more precise form for the $2 \times 2$ blocks of the full SLG-ring Hamiltonian in the narrow-width limit is

$$
\begin{aligned}
\frac{\mathrm{H}_{\mathrm{SLG}}^{(\tau, n)}}{\hbar v / W}=\pi\left(n-\frac{1}{2}\right)\left[\sigma_{3}+\frac{2}{\pi(2 n-1)} \frac{W}{R} l \sigma_{1}\right. \\
\left.\quad-\tau \frac{4}{\pi^{3}(2 n-1)^{3}}\left(\frac{W}{R}\right)^{2} l \sigma_{0}\right],
\end{aligned}
$$


which yields the dispersions

$$
\frac{E_{\mathrm{SLG}}^{(\tau, \pm n)}(l)}{\hbar v / W}=-\tau \frac{2}{\pi^{2}(2 n-1)^{2}}\left(\frac{W}{R}\right)^{2} l \pm \sqrt{\pi^{2}\left(n-\frac{1}{2}\right)^{2}+\left(\frac{W}{R}\right)^{2}} l^{2}
$$

which can be determined using Eqn. 2.26). The expansion of Eqn. (3.33) to leading (i.e., quadratic) order in $\epsilon$ agrees fully with Eqn. (8) of Ref. [5] that was obtained under similar assumptions.

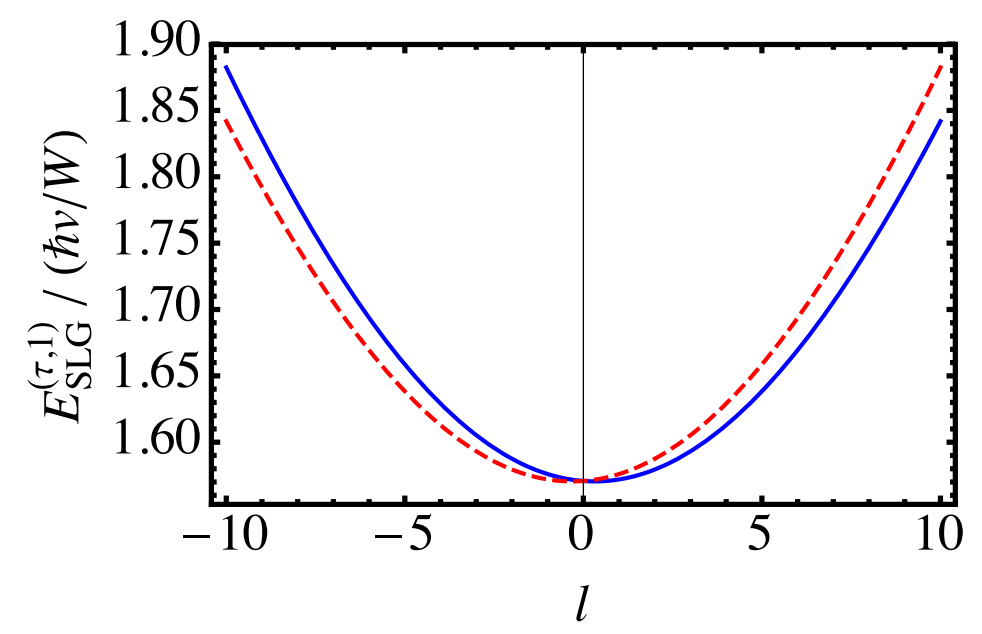

Figure 3.2: Lowest-subband dispersion for a single-layer-graphene ring with aspect ratio $W / R=0.1$. The solid blue (dashed red) curve corresponds to the $\mathbf{K}\left(\mathbf{K}^{\prime}\right)$ valley.

Fig. (3.2) shows the dispersion for the lowest $(n=1)$ subband according to Eqn. 3.33 for a particular value of the ring aspect ratio $W / R$. The asymmetry with respect to inversion of the total-angular-momentum quantum number $(l \rightarrow-l)$ for a given valley is clearly visible. However, due to time-reversal symmetry, the dispersions for the $\mathbf{K}$ and $\mathbf{K}^{\prime}$-valley degrees of freedom are related via the combined transformation $l \rightarrow-l$ and $\tau \rightarrow-\tau$. The two curves form the famous Kramer pairs which, in this case, are not topologically protected since $E_{\mathrm{SLG}, 0}^{(\tau, 1)}>\left|V_{0}\right|=0$. The relative shift of the dispersion curves can be estimated as $2 /[\pi(2 n-1)]$, which is a decreasing function of the subband index $n$. This shift is explored further in the Chapter 6 .

Now that we have finished our analysis of the $V_{0}=0$ SLG QR system, we move on to understanding the dispersions of a general $V_{0}$ mass-confinement in SLG and TMD QR systems. First, we study the general mass-confinement 
of SLG QRs and determine the azimuthal dependence of the energy subband dispersion and discover the swapping of relative positions of the $\tau$-valley dispersions. Then, we study the changes of the dispersion in the presence of the spin-splitting for TMD QRs with a particular focus on molybdenum diselenide $\left(\mathrm{MoSe}_{2}\right)$.

\subsection{General mass-confinement}

\subsubsection{Single-layer graphene}

Mass-gaps in SLG sheets are created due to the presence of certain disorders and substrate interactions. Hence it is realistic to examine the system with a general $V_{0}$ confinement value. The same symmetries for $H_{\mathrm{SLG}, 1}^{(\tau)}$ hold as in the massless-Dirac case and are given by Eqns. (3.20) and (3.21). Thus the $\mathrm{H}_{\mathrm{SLG}, 1}^{(\tau)}$ matrix elements are given by

$$
\begin{aligned}
\mathrm{h} H_{\mathrm{SLG}, 1}^{(\tau)} \mathrm{i}_{\tau, \mathrm{SLG}}^{\left(n_{a}, n_{a}^{\prime}\right)}=\int_{R-\frac{W}{2}}^{R+\frac{W}{2}} \phi_{\mathrm{SLG}}^{\left(\tau, n_{a}\right)^{\dagger}} H_{\mathrm{SLG}, 1}^{(\tau)} \phi_{\mathrm{SLG}}^{\left(\tau, n_{a}^{\prime}\right)} 2 \pi r d r \\
=\mathrm{N}_{n_{a}} \mathrm{~N}_{n_{a}^{\prime}} \tau \int_{R-\frac{W}{2}}^{R+\frac{W}{2}} \hbar v \frac{l}{r}\left(\gamma_{n_{a}^{\prime}} \sin \left(\kappa_{n_{a}^{\prime}}(r-R)\right) \cos \left(\kappa_{n_{a}}(r-R)\right)\right. \\
\left.\quad+\gamma_{n_{a}} \sin \left(\kappa_{n_{a}}(r-R)\right) \cos \left(\kappa_{n_{a}^{\prime}}(r-R)\right)\right) d r
\end{aligned}
$$

and

$$
\begin{aligned}
\mathrm{h} H_{\mathrm{SLG}, 1}^{(\tau)} \sigma_{2} \mathrm{i}_{\tau, \mathrm{SLG}}^{\left(n_{a}, n_{a}^{\prime}\right)}=\int_{R-\frac{W}{2}}^{R+\frac{W}{2}} \phi_{\mathrm{SLG}}^{\left(\tau, n_{a}\right)^{\dagger}} H_{\mathrm{SLG}, 1}^{(\tau)} \sigma_{2} \phi_{\mathrm{SLG}}^{\left(\tau, n_{a}^{\prime}\right)} 2 \pi r d r \\
=\mathrm{N}_{n_{a}} \mathrm{~N}_{n_{a}^{\prime}} \int_{R-\frac{W}{2}}^{R+\frac{W}{2}} \hbar v \frac{l}{r}\left(\cos \left(\kappa_{n_{a}}(r-R)\right) \cos \left(\kappa_{n_{a}^{\prime}}(r-R)\right)\right. \\
\left.\quad+\gamma_{n_{a}} \gamma_{n_{a}^{\prime}} \sin \left(\kappa_{n_{a}}(r-R)\right) \sin \left(\kappa_{n_{a}^{\prime}}(r-R)\right)\right) d r
\end{aligned}
$$

The solutions to these integrals, via a series-expansion up to the second order of $\epsilon$, can be found in Appendix (B). Using these solutions and applying the knowledge that we have gained from Sec. (3.2), the accurate subband 
dispersion up to order $\epsilon^{2}$ is given by the $2 \times 2$ Hamiltonian [as in Eqn. (2.25)]:

$$
\mathrm{H}_{\mathrm{SLG}}^{(\tau, n)}=\frac{\hbar v}{W}\left[\frac{E_{\mathrm{SLG}, 0}^{(\tau, n)}}{\hbar v / W} \sigma_{3}-\tau\left(\frac{W}{R}\right)^{2} l \Gamma_{\mathrm{SLG}} \sigma_{0}+\frac{W}{R} l \sigma_{1}\right]
$$

where $E_{\mathrm{SLG}, 0}^{(\tau, n)}$ is found using the secular equation given by Eqn. 3.10 . $\Gamma_{\alpha}$ is given by

$$
\Gamma_{\alpha}=\frac{1}{2} \frac{1+\frac{V_{0}}{\hbar v / W}}{\left(\frac{E_{\alpha, 0}^{(\tau, n)}}{\hbar v / W}\right)^{2}+\frac{V_{0}}{\hbar v / W}} .
$$

where $\alpha=\{\mathrm{SLG}, \mathrm{TMD}\}$ in this instance. The general subband energy dispersion, $E_{\mathrm{SLG}}^{(\tau, n)}(l)$, can be found to be

$$
\frac{E_{\mathrm{SLG}}^{(\tau, \pm n)}(l)}{\hbar v / W}=-\tau\left(\frac{W}{R}\right)^{2} l \Gamma_{\mathrm{SLG}} \pm \sqrt{\left[\frac{E_{\mathrm{SLG}, 0}^{(\tau, n)}}{\hbar v / W}\right]^{2}+\left(\frac{W}{R}\right)^{2} l^{2}} .
$$

This is naturally equivalent to Eqn. (3.33) when $V_{0}=0$. Once again we can see that for a given $l, E_{\mathrm{SLG}}^{(\tau, \pm n)}(l) /(\hbar v / W)$ varies universally with parameter $V_{0} /(\hbar v / W)$. We choose two $V_{0} /(\hbar v / W)$ values: $V_{0} /(\hbar v / W)=-2$ (with TPES) and $V_{0} /(\hbar v / W)=0$ (trivial case) and plot the the lowest two subbands $(n=1,2)$ in Fig 3.3 .

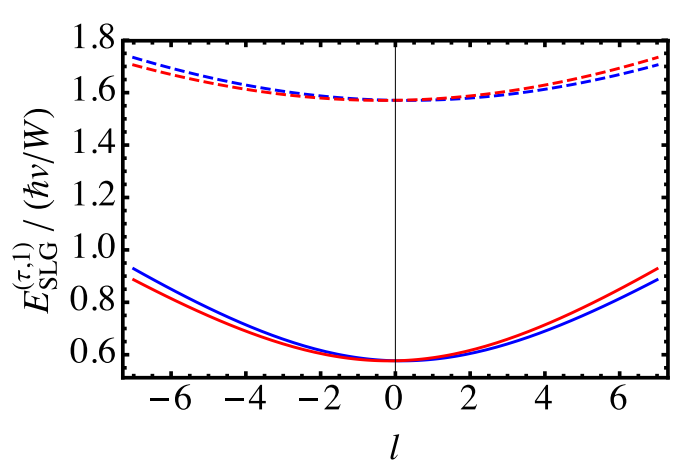

(a) Lowest subband

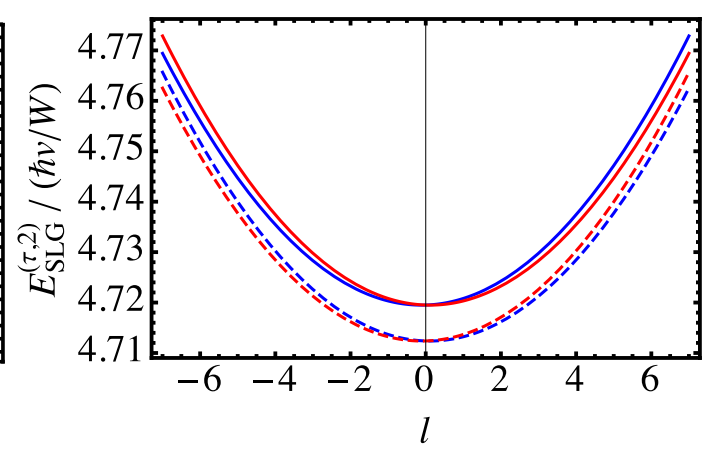

(b) Second-lowest subband

Figure 3.3: Plot of general subband energy dispersion of lowest (a) and second-lowest (b) subbands for $W / R=0.1, V_{0} /(\hbar v / W)=-2$ (solid) and 0 (dashed) with both isospin valleys $\mathbf{K}$ (blue) and $\mathbf{K}^{\prime}$ (red).

We see that for $n=1$ at any given energy, the $\tau=1$ curve is shifted to the right of the $\tau=-1$ curve for both the trivial and topological phases of the 
QR. However for $n=2$, the $\tau=1$ curve shifts from the right to the left of the $\tau=-1$ curve as we switch from the trivial to the topological phase. This difference highlights some of the phenomena that happen when we transition from the trivial to the topological phase of the material.

To understand this more, we have to understand the component $\Gamma_{\mathrm{SLG}}$ which governs this shift. We plot $\Gamma_{\mathrm{SLG}}$ as a function of $V_{0} /(\hbar v / W)$ for the three lowest subbands in Fig. (3.4). For $n>1$, we have $\Gamma_{\mathrm{SLG}}=0$ when $V_{0} /(\hbar v / W)=$ -1 , i.e. the transition point between topological and trivial regimes [Eqn. (3.13)].

This means that there is no shift between the isospin valley curves at that parameter value. Thus, since the shift between isospin valleys for $n>1$ monotonically increases with increasing $V_{0} /(\hbar v / W) 2$, there is a distinct difference in the relative positions of the isospin valley subbands between the trivial and topological phase of the material.

However, the $n=1$ subband behaves significantly different because there is no such transition, as can be seen in Fig. (3.4). Additionally, the $\Gamma_{\mathrm{SLG}}$ factor monotonically decreases as a function of $V_{0} /(\hbar v / W)$. These features may be a hallmark of the topological nature and protection of this state. All of these concepts will be discussed further in Chapter 6 where we will see that the Berry phase is also affected by the shifts of $\pm \tau$ energy dispersions.

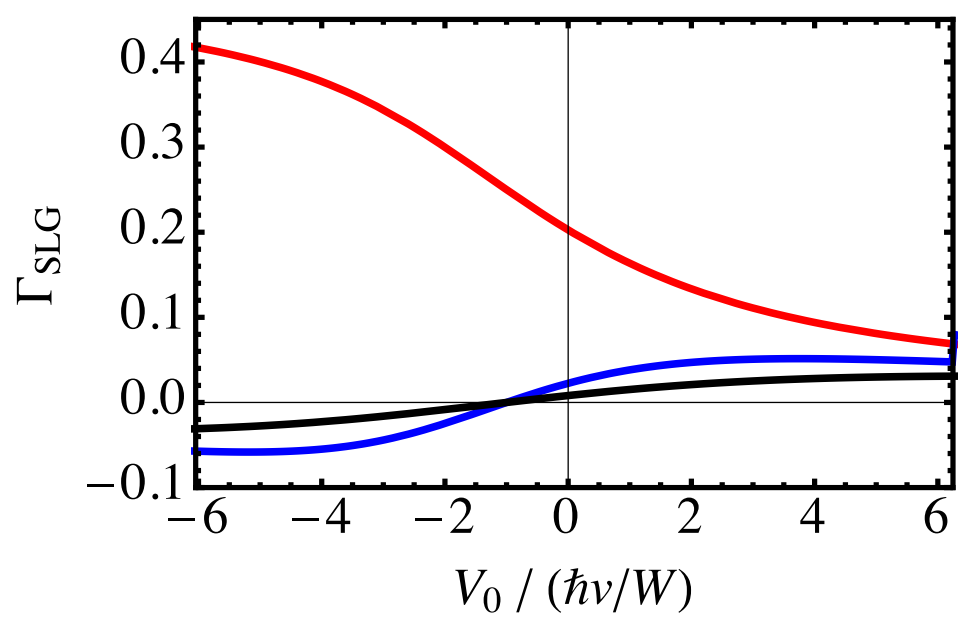

Figure 3.4: Plot of $\Gamma_{\mathrm{SLG}}$ as a function of $V_{0} /(\hbar v / W)$ for the different subbands: $n=1$ (red), 2 (blue) and 3 (black). Only $n>1$ subbands cross at $V_{0} /(\hbar v / W)=-1$ from negative to positive values of $\Gamma_{\mathrm{SLG}}$.

\footnotetext{
${ }^{2}$ This is because the shift depends on $\Gamma_{\mathrm{SLG}}$ [as given in Eqn. (3.37)] which also increases monotonically for an increasing $V_{0} /(\hbar v / W)$. This we will definitively show in Chapter 6
} 
We will now proceed to analyse the general mass-confinement subband energy dispersion of single-layer TMD rings. Mathematically, the calculations that arise from this case are extremely similar to those in SLG. Nevertheless, we give a somewhat simplified, general description of the system including the most essential calculation details.

\subsubsection{Transition-metal dichalcogenides}

Single-layer TMD rings have the same form of the radial equation as the SLG rings - however the effective spin-dependent potential $V_{0} \equiv \frac{1}{2}\left(\Delta-\lambda \tau s_{3}\right)$ is defined such that there is never a massless limit in TMD systems $3^{3}$. The gap parameter $V_{0}$ effectively acts as though there are two independent gaps for $\tau s_{3}= \pm 1$, respectively. Unlike the SLG system that is confinementdominated (i.e., generally has $\hbar v / W>V_{0}$ ), the large value of $\Delta \sim \hbar v / 1.81 \AA$ makes the TMD system gap dominated [19].

In TMD, the form of the wavefunctions remain unchanged and hence we can use Eqns. (3.16a) and (3.16b) as our base wavefunctions for finding the matrix elements for $\mathrm{H}_{\mathrm{TMD}, 1}^{(\tau)}$. Recall that $H_{\mathrm{TMD}, 1}^{(\tau)}$ is of the form

$$
H_{\mathrm{TMD}, 1}^{(\tau)}=\hbar v \frac{l}{r} \sigma_{2}+\frac{\lambda}{2} \tau s_{3} \sigma_{0}
$$

with the extra $\sigma_{0}$ term as compared to SLG which is just an energy shift with respect to the $\tau s_{3}$ factor in the overall energy dispersion. Once again, the symmetries are given by

$$
\mathrm{h} H_{\mathrm{TMD}, 1}^{(\tau)} \mathrm{i}_{\tau, \mathrm{TMD}}^{\left(n, n^{\prime}\right)}=\mathrm{h}_{2} H_{\mathrm{TMD}, 1}^{(\tau)} \sigma_{2} \mathrm{i}_{\tau, \mathrm{TMD}}^{\left(n, n^{\prime}\right)}
$$

and

$$
\mathrm{h}_{2} H_{\mathrm{TMD}, 1}^{(\tau)} \mathrm{i}_{\tau, \mathrm{TMD}}^{\left(n, n^{\prime}\right)}=\mathrm{h} H_{\mathrm{TMD}, 1}^{(\tau)} \sigma_{2} \mathbf{i}_{\tau, \mathrm{TMD}}^{\left(n, n^{\prime}\right)}
$$

which hold due to the relation $\sigma_{2}^{2}=\sigma_{0}$. The matrix elements are thus given

\footnotetext{
${ }^{3}$ Theoretically, there could be a massless-Dirac limit if we could impose a variable mass confinement that can effectively cancel the $\frac{1}{2}\left(\Delta-\lambda \tau s_{3}\right)$ potential. However this does not seem to be a viable option yet.
} 
by

$$
\mathrm{h} H_{\mathrm{TMD}, 1}^{(\tau)} \mathrm{i}_{\tau, \mathrm{TMD}}^{\left(n_{a}, n_{a}^{\prime}\right)}=\mathrm{h} H_{\mathrm{SLG}, 1}^{(\tau)} \mathrm{i}_{\tau, \mathrm{SLG}}^{\left(n_{a}, n_{a}^{\prime}\right)}+\frac{\lambda}{2} \tau s_{3} \delta_{n_{a} n_{a}^{\prime}}
$$

and

$$
\mathrm{h} H_{\mathrm{TMD}, 1}^{(\tau)} \sigma_{2} \mathrm{i}_{\tau, \mathrm{TMD}}^{\left(n_{a}, n_{a}^{\prime}\right)}=\mathrm{h} H_{\mathrm{SLG}, 1}^{(\tau)} \sigma_{2} \mathrm{i}_{\tau, \mathrm{SLG}}^{\left(n_{a}, n_{a}^{\prime}\right)}
$$

As in the case of SLG, using these solutions, we find that the subband dispersion up to order $\epsilon^{2}$ is accurately described by the effective $2 \times 2$ Hamiltonian:

$$
\mathrm{H}_{\mathrm{TMD}}^{(\tau, n)}=\frac{\hbar v}{W}\left[\frac{E_{\mathrm{TMD}, 0}^{(\tau, n)}}{\hbar v / W} \sigma_{3}-\tau\left(\frac{W}{R}\right)^{2} l \Gamma_{\mathrm{TMD}} \sigma_{0}+\frac{W}{R} l \sigma_{1}+\frac{\lambda}{2 \hbar v / W} \tau s_{3}\right]
$$

where $E_{\mathrm{TMD}, 0}^{(\tau, n)}$ is the energy that is derived using the secular equation, with the altered potential that is now spin-dependent, as given by Eqn. (3.10). The general subband energy dispersion, $E_{\mathrm{TMD}}^{(\tau, n)}(l)$ is found to be

$$
\frac{E_{\mathrm{TMD}}^{(\tau, \pm n)}(l)}{\hbar v / W}=-\tau\left(\frac{W}{R}\right)^{2} l \Gamma_{\mathrm{TMD}}+\frac{\lambda}{2 \hbar v / W} \tau s_{3} \pm \sqrt{\left[\frac{E_{\mathrm{TMD}, 0}^{(\tau, n)}}{\hbar v / W}\right]^{2}+\left(\frac{W}{R}\right)^{2} l^{2}}
$$

which is equivalent to the SLG general mass-confinement subband energy dispersion when $\lambda=0$ [see Eqn. (3.38)]. We see that the only change, for TMD rings, is that the energy dispersion includes the real-spin dependence term which causes the energy to receive a $\pm \lambda / 2$ energy shift based on the sign of $\tau s_{3}$.

Generally accepted values for group-VI dichalcogenides of the valence band spin-splitting strength $\lambda$ range around the hundreds of meV 4 , while band gap energies range around a couple of eV [19]. This means that for all TMDs, $V_{0}>0$ always holds because the band gap energy is much larger than the valence and conduction band spin-splitting. Thus, without a variable mass confinement, there are never TPESs in this system.

Using an experimental values for $\mathrm{MoSe}_{2}$ rings, with $\lambda=-90 \mathrm{meV}$ 22, 69,

\footnotetext{
${ }^{4}$ For conduction bands, this intrinsic spin-splitting is even smaller, being on the order of magnitude around $\sim 10 \mathrm{meV}$.
} 
$\hbar v=253 \mathrm{meV} \mathrm{nm}$ and $\Delta=1.40 \mathrm{eV}[19]$, we plot Fig. (3.5) which shows the characteristic spin dependent splitting. We can also observe that the timereversal symmetry between the two isospin valley dispersions in the case of TMD is different from that of SLG. To get the time-reversal partner, we have to use the transformation $\tau \rightarrow-\tau, s_{3} \rightarrow-s_{3}$ (as compared to just $\tau \rightarrow-\tau$ in SLG) and $l \rightarrow-l$. Additionally, given our initial model, we assume that intrinsic spin-splitting of the conduction bands is zero thus resulting in a negligible spin-splitting of the $n=1$ band. However in Fig. (3.5a), we can see a minor spin-splitting in the conduction band that is due to ring confinement, thus allowing us to see a deviation between the $\tau s_{3}= \pm 1$ subbands.

If we were able to vary the mass-confinement, we would see very similar results as in Sec. (3.3.1) for SLG, with the addition of spin splitting. In fact, there would be situations where one $\tau s_{3}$ state is a TPES while the $-\tau s_{3}$ state is trivial. For example this would happen if $V_{0}=-1-\lambda \tau s_{3} / 2$ where we have shifted the mass-confinement by a factor $-\frac{1}{2} \Delta-1$.

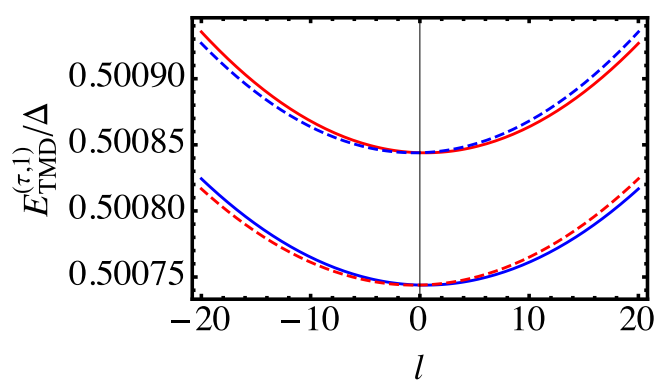

(a) Conduction-band states

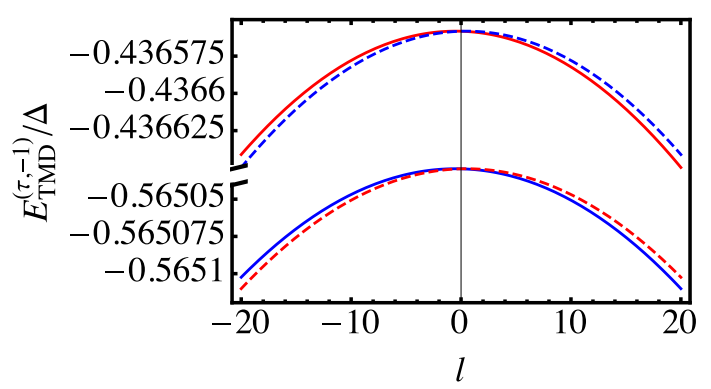

(b) Valence-band states

Figure 3.5: Lowest-subband dispersion relations for an $\mathrm{MoSe}_{2} Q R$ with spin-dependent splitting ( $\uparrow$ blue, $\downarrow$ red) for isospin valley $\mathbf{K}$ and $\mathbf{K}^{\prime}$ (solid and dashed respectively). (a) Figure of the conduction band where the lower and upper bands have $\tau s_{3}=1$ and -1 , respectively. We see minor conduction band $\tau s_{3}$ spin-splitting. (b) Figure of the valence bands where the lower and upper bands have $\tau s_{3}=1$ and -1 , respectively. The spin-splitting direction is in agreement with Ref. [19]. The parameter values are given by $\lambda=-90 \mathrm{meV}$, $\hbar v=253 \mathrm{meV} \mathrm{nm}$ and $\Delta=1.40 \mathrm{eV}, W=40 \mathrm{~nm}$ and $R=400 \mathrm{~nm}$.

We have now wrapped up our description of SLG and TMD ring confinement effects and dispersions. The transport properties related to these results are explored further in the Chapters 5 and 6. Using the analysis from this chapter, we have gained a better understanding of simple ring-confined Dirac systems which helps us tackle the more difficult case of the narrow-gapped 
semiconductor quantum well, which is presented in the next chapter. 


\section{Chapter 4}

\section{Semiconductor quantum well}

In this chapter we look into the energy dispersions of a narrow-gap semiconductor quantum well (SQW) ring using the methodology described in Sec. (2.2).

Similarly to single-layer graphene (SLG), we map $H_{\mathrm{SQW}, 0}^{(\tau)}$ onto a 1D Diraclike equation to which we apply a hard-wall confinement. The eigenenergies and eigenstates are then determined and found to be in agreement with previous literature [7]. Then we explore the properties associated with the transition between trivial and topological phases, and the effects of changing the system parameters of the quantum well. Next, for a general strength of mass-confinement, we determine the angular momentum dependence of SQW rings by finding the matrix elements of $\mathrm{H}_{\mathrm{SQW}, 1}^{(\tau)}$. As in the SLG chapter, we use the approximation that the ring aspect ratio is small, which allows us to determine the explicit form of the subband energy dispersion as a function of angular momentum quantum number, $l$. Finally, we examine the relative positions of the $\tau$ flavour energy dispersions and find qualitative differences between the lowest-subband and higher subbands that indicate the topological nature of the lowest-subband. 


\subsection{Ring structures in SQWs}

We start with the same Ansatz as in SLG:

$$
\phi_{\mathrm{SQW}}^{(\tau, n)}(r)=\frac{\chi_{\mathrm{SQW}}^{(\tau, n)}(r)}{\overline{2 \pi r}},
$$

with the relations

$$
\begin{aligned}
& k_{r}\left(\frac{\chi_{\mathrm{S} Q W}^{(\tau, n)}(r)}{\overline{2 \pi r}}\right) \equiv \frac{1}{\overline{2 \pi r}}\left(-i \partial_{r}\right) \chi_{\mathrm{SQW}}^{(\tau, n)}(r), \\
& k_{r}^{2}\left(\frac{\chi_{\mathrm{SQW}}^{(\tau, n)}(r)}{\overline{2 \pi r}}\right) \equiv \sqrt{\frac{1}{2 \pi r}}\left(-\partial_{r}^{2}\right) \chi_{\mathrm{SQW}}^{(\tau, n)}(r),
\end{aligned}
$$

which were derived with the use of Eqns. 2.10c and 2.10a. Hence the radial Eqn. 2.17a arising from the SQW model becomes

$$
\begin{aligned}
-i \frac{d \chi_{\mathrm{SQW}}^{(\tau, n)}}{d r} \tau \sigma_{1} & +\frac{B}{\hbar v} \frac{d^{2} \chi_{\mathrm{SQW}}^{(\tau, n)}}{d r^{2}} \sigma_{3} \\
& +\frac{1}{\hbar v}\left[\tilde{V}(r) \sigma_{3}-\left(E_{\mathrm{SQW}, 0}^{(\tau, n)}-D \frac{d^{2}}{d r^{2}}\right) \sigma_{0}\right] \chi_{\mathrm{SQW}}^{(\tau, n)}=0 .
\end{aligned}
$$

For $\tilde{V}(r) \equiv M$, Eqn. 4.3 constitutes the 1D Dirac equation with the addition of the $\partial_{r}^{2}$ terms. We apply a hard-wall confinement of the form

$$
\tilde{V}(r)= \begin{cases}M & \text { for } r_{\mathrm{i}}<r<r_{\mathrm{o}} \\ \infty & \text { elsewhere }\end{cases}
$$

where $r_{i}$ and $r_{o}$ are the inner and outer radii. We also define the width $W$ and radius $R$ as in the SLG chapter [see Eqn. (3.5)]. We remind the reader that $M$ is the variable band gap parameter which governs the topological nature of the material, as discussed in Chapter 1. In the inverted regime, i.e. $M<0$, we expect TPES in the form of evanescent eigenstates to appear.

Using the units $E_{0} \equiv(\hbar v)^{2} /|B|$ and $q_{0} \equiv \hbar v /|B|$ for energy and momentum, respectively, we express our system parameters as: $\tilde{D} \equiv D /|B|, \tilde{E} \equiv$ $E_{\mathrm{SQW}, 0}^{(\tau, n)} / E_{0}, \tilde{E}_{k} \equiv\left(E_{\mathrm{SQW}, 0}^{(\tau, n)}+D k^{2}\right) / E_{0}$, and $\tilde{k} \equiv k / q_{0}$. We also redefine the 
band gap as $\xi_{\mathrm{M}} \equiv M / E_{0}$. Using these new definitions, the energy eigenvalues and corresponding eigenstates of Eqn. 4.3 can be written as

$$
\begin{aligned}
\tilde{E}_{k}^{ \pm} & = \pm \sqrt{\tilde{k}^{4}+\left(2 \xi_{\mathrm{M}}+1\right) \tilde{k}^{2}+\xi_{\mathrm{M}}^{2}}, \\
\chi_{\mathrm{SQW}}(r) & =\left(\begin{array}{c}
1 \\
\tau \operatorname{sgn}(\tilde{k}) \operatorname{sgn}\left(\tilde{E}+\xi_{\mathrm{M}}+\tilde{k}^{2}\right) \gamma
\end{array}\right) \mathrm{e}^{i \tilde{\kappa} \tilde{r}},
\end{aligned}
$$

where $\operatorname{sgn}(\alpha) \equiv \alpha / \sqrt{\alpha^{2}}$ and we use the fact that $B<0$ in HgTe $[20$. We also introduce the abbreviation

$$
\gamma=\sqrt{\frac{\tilde{E}_{k}-\xi_{\mathrm{M}}-\tilde{k}^{2}}{\tilde{E}_{k}+\xi_{\mathrm{M}}+\tilde{k}^{2}}} .
$$

The form of this wavefunction is identical to that in Eqn. (1.5) for 1D motion $(\mathbf{p}= \pm \hbar k)$. Refer to Appendix A.1 for the relevant $\operatorname{signs}$ of $\operatorname{sgn}\left(\tilde{E}+\xi_{\mathrm{M}}+\tilde{k}^{2}\right)$ which will play an important role in the hard-wall confined secular equation.

To find the bound states of the 1D hard-wall confined SQW model, we superimpose possible solutions for given energy $\tilde{E}$ and determine the coefficients from the boundary conditions. This is done by solving for the possible $\tilde{k}$ solution in the secular equation [Eqn. 4.5a)]. There are four possible values of the wave number at fixed $\tilde{E} \geq\left|\xi_{\mathrm{M}}\right|$ and $\tilde{E} \leq-\left|\xi_{\mathrm{M}}\right|$, with two of them being real and given by $\tilde{k}= \pm \tilde{\kappa}$, and two imaginary ones given by $\tilde{k}= \pm i \tilde{\lambda}$ where $\tilde{\kappa}=\kappa / q_{0}$ and $\tilde{\lambda}=\lambda / q_{0}$. We find

$$
\begin{aligned}
\tilde{\kappa} & =\left[\frac{1}{2}\left(\sqrt{\left(2 \xi_{\mathrm{M}}+1\right)^{2}+4\left(\tilde{E}_{\kappa}^{2}-\xi_{\mathrm{M}}^{2}\right)}-1\right)-\xi_{\mathrm{M}}\right]^{\frac{1}{2}}, \\
& =\left[\frac{1}{2}\left(\sqrt{1+4 \xi_{\mathrm{M}}+4 \tilde{E}_{\kappa}^{2}}-1\right)-\xi_{\mathrm{M}}\right]^{\frac{1}{2}}, \\
\tilde{\lambda} & =\left[\frac{1}{2}\left(\sqrt{\left(2 \xi_{M}+1\right)^{2}+4\left(\tilde{E}_{i \lambda}^{2}-\xi_{\mathrm{M}}^{2}\right)}+1\right)+\xi_{\mathrm{M}}\right]^{\frac{1}{2}}, \\
& =\left[\frac{1}{2}\left(\sqrt{1+4 \xi_{\mathrm{M}}+4 \tilde{E}_{i \lambda}^{2}}+1\right)+\xi_{\mathrm{M}}\right]^{\frac{1}{2}} .
\end{aligned}
$$


The full Ansatz for the bound-state wave function is

$$
\begin{array}{r}
\chi_{\mathrm{SQW}}^{(\tau, n)}(r)=c_{1 \kappa}^{(\tau, n)}\left(\begin{array}{c}
1 \\
\tau \sigma \gamma_{\kappa}
\end{array}\right) \mathrm{e}^{i \kappa r}+c_{2 \kappa}^{(\tau, n)}\left(\begin{array}{c}
1 \\
-\tau \sigma \gamma_{\kappa}
\end{array}\right) \mathrm{e}^{-i \kappa r} \\
+c_{1 \lambda}^{(\tau, n)}\left(\begin{array}{c}
\bar{\gamma}_{\lambda} \\
-i \tau
\end{array}\right) \mathrm{e}^{-\lambda r}+c_{2 \lambda}^{(\tau, n)}\left(\begin{array}{c}
\bar{\gamma}_{\lambda} \\
i \tau
\end{array}\right) \mathrm{e}^{\lambda r},
\end{array}
$$

where $\sigma \equiv \operatorname{sgn}\left(\tilde{E}+\xi_{M}+\tilde{\kappa}^{2}\right)$. The parameters are given by

$$
\begin{aligned}
& \gamma_{\kappa}=\left[\frac{\tilde{E}_{\kappa}-\frac{1}{2}\left(\sqrt{1+4 \xi_{\mathrm{M}}+4 \tilde{E}_{\kappa}^{2}}-1\right)}{\tilde{E}_{\kappa}+\frac{1}{2}\left(\sqrt{1+4 \xi_{\mathrm{M}}+4 \tilde{E}_{\kappa}^{2}}-1\right)}\right]^{\frac{1}{2}}, \\
& \bar{\gamma}_{\lambda}=\left[\frac{\frac{1}{2}\left(\sqrt{1+4 \xi_{\mathrm{M}}+4 \tilde{E}_{i \lambda}^{2}}+1\right)-\tilde{E}_{i \lambda}}{\frac{1}{2}\left(\sqrt{1+4 \xi_{\mathrm{M}}+4 \tilde{E}_{i \lambda}^{2}}+1\right)+\tilde{E}_{i \lambda}}\right]^{\frac{1}{2}} .
\end{aligned}
$$

There will generally be many solutions with $|\tilde{E}| \geq\left|\xi_{\mathrm{M}}\right|$ that are bulk-derived ring-confined states. In addition, we expect solutions to arise with $|\tilde{E}|<\left|\xi_{\mathrm{M}}\right|$, at least in the inverted regime (i.e. $\xi_{\mathrm{M}}<0$ ), due to the hybridisation of edge states from the inner and outer boundaries. In the latter situation, we have a fully evanescent state with $\tilde{\kappa} \equiv i \tilde{\bar{\kappa}}$ and $\gamma_{\kappa} \equiv i \bar{\gamma}_{\bar{\kappa}}$, where

$$
\begin{gathered}
\tilde{\bar{\kappa}}=\left[\xi_{\mathrm{M}}-\frac{1}{2}\left(\sqrt{1+4 \xi_{\mathrm{M}}+4 \tilde{E}_{i \bar{\kappa}}^{2}}-1\right)\right]^{\frac{1}{2}}, \\
\bar{\gamma}_{\bar{\kappa}}=\left[\frac{\frac{1}{2}\left(\sqrt{1+4 \xi_{\mathrm{M}}+4 \tilde{E}_{i \bar{\kappa}}^{2}}-1\right)-\tilde{E}_{i \bar{\kappa}}}{\frac{1}{2}\left(\sqrt{1+4 \xi_{\mathrm{M}}+4 \tilde{E}_{i \bar{\kappa}}^{2}}-1\right)+\tilde{E}_{i \bar{\kappa}}}\right]^{\frac{1}{2}}
\end{gathered}
$$

are then real numbers for all values of $\tilde{E}$ and $\tilde{D}$ within the gap when $\xi_{\mathrm{M}} \geq$ $-1 / 4$. When $\xi_{\mathrm{M}}<-1 / 4, \tilde{\bar{\kappa}}$ and $\bar{\gamma}_{\bar{\kappa}}$ are not guaranteed to be real and may become a complex quantity with both a real and imaginary component. This is due to the square-root values (such as $\sqrt{1+4 \xi_{\mathrm{M}}+4 \tilde{E}_{i \bar{\kappa}}^{2}}$ in $\tilde{\bar{\kappa}}$ ) that appear in all the $\tilde{\kappa}, \tilde{\lambda}$ and $\gamma$ components which may become imaginary in this limit. The exact limit at which it becomes imaginary depends on the specific 
configurations of $\tilde{D}$ and $\tilde{E}$ and can be shown to be

$$
\xi_{\mathrm{M}} \leq \frac{-1+2 \tilde{D} \tilde{E}+\sqrt{\left(\tilde{D}^{2}-1\right)(4 \tilde{D} \tilde{E}-1)}}{2 \tilde{D}^{2}}
$$

Henceforth, unless otherwise mentioned, we only consider gap values that obey the strict limit of $\xi_{\mathrm{M}}>-1 / 4$. We now move on to derive the secular energy equation and explore its solutions.

\subsubsection{Secular energy solutions}

We treat the hard-wall ring confinement conventionally by introducing the open boundary conditions 6, 7. $\chi_{\mathrm{SQW}}^{(\tau, n)}\left(r_{\mathrm{i}, \mathrm{o}}\right)=0$. Straightforward calculation yields the secular equation

$$
\frac{\sin (\tilde{\kappa} \tilde{W}) \sinh (\tilde{\lambda} \tilde{W})}{1-\cos (\tilde{\kappa} \tilde{W}) \cosh (\tilde{\lambda} \tilde{W})}=\sigma \frac{2 \bar{\gamma}_{\lambda} \gamma_{\kappa}}{1-\bar{\gamma}_{\lambda}^{2} \gamma_{\kappa}^{2}}
$$

where $\tilde{W} \equiv q_{0} W$. We rearrange this using trigonometric identities to obtain

$$
\frac{\tanh \left(\frac{\tilde{\lambda} \tilde{W}}{2}\right)}{\tan \left(\frac{\tilde{\kappa} \tilde{W}}{2}\right)}-\frac{\tan \left(\frac{\tilde{\kappa} \tilde{W}}{2}\right)}{\tanh \left(\frac{\tilde{\lambda} \tilde{W}}{2}\right)}=\sigma \frac{\bar{\gamma}_{\tilde{\lambda}}^{2} \gamma_{\tilde{\kappa}}^{2}-1}{\bar{\gamma}_{\tilde{\lambda}} \gamma_{\tilde{\kappa}}}
$$

which can be shown to be equivalent to Eqn. (9) in Ref. [7]. This derivation is shown in Appendix A.2. Interestingly, in the limit $\tilde{\lambda} \tilde{W} \quad 1$ and $\tilde{\lambda}>\tilde{\kappa}$, which are generally satisfied 58 except potentially very deep in the inverted regime, Eqn. 4.11 simplifies to

$$
\tan (\tilde{\kappa} \tilde{W})=\sigma \frac{2 \bar{\gamma}_{\lambda} \gamma_{\kappa}}{\bar{\gamma}_{\lambda}^{2} \gamma_{\kappa}^{2}-1}
$$

This has the same structure as the secular Eqn. (3.10) for a confined 1D Dirac particle with $\gamma \rightarrow \bar{\gamma}_{\lambda} \gamma_{\kappa}$. Bound states arising from the hybridisation of edge states in the inverted regime [7] have energies satisfying

$$
\tanh (\tilde{\bar{\kappa}} \tilde{W})=-\sigma \frac{2 \bar{\gamma}_{\lambda} \bar{\gamma}_{\bar{\kappa}}}{\bar{\gamma}_{\lambda}^{2} \bar{\gamma}_{\bar{\kappa}}^{2}+1}
$$

Analogous to the SLG case, we find that the secular equation given by 
Eqn. 4.12 has two distinct energy solutions that arise from the underlying symmetry of the system. These two forms are given by:

$$
\sigma \gamma_{\tilde{\kappa}} \bar{\gamma}_{\tilde{\lambda}}=\left\{\begin{array}{r}
\frac{\cot \left(\frac{\tilde{\kappa} \tilde{W}}{2}\right)}{\operatorname{coth}\left(\frac{\tilde{\lambda} \tilde{W}}{2}\right)} \\
-\frac{\tan \left(\frac{\tilde{\kappa} \tilde{W}}{2}\right)}{\tanh \left(\frac{\tilde{\lambda} \tilde{W}}{2}\right)}
\end{array}\right.
$$

which is very similar to the SLG case [Eqns. (3.11a) and (3.11b]). Solutions to Eqn. 4.15a correspond to the odd $n>0$ states and Eqn. 4.15b to the even $n>0$ states and vice-versa for $n<0$. Thus adjacent energy states are always of different "types". Henceforth, we shall refer to states with energies determined by Eqn. 4.15a as type (a) and Eqn. 4.15b as type (b).

We can rearrange Eqns. 4.15a and 4.15b for $\gamma_{\kappa}$ and $\bar{\gamma}_{\lambda}$ terms, respectively, which will come in handy for later reference. We find

$$
\sigma \gamma_{\kappa}=\frac{\left(\tilde{\kappa}^{2}+\tilde{\lambda}^{2}\right) \cot \left(\frac{\tilde{\kappa} \tilde{W}}{2}\right)}{\tilde{\lambda} \operatorname{coth}\left(\frac{\tilde{\lambda} \tilde{W}}{2}\right)-\tilde{\kappa} \cot \left(\frac{\tilde{\kappa} \tilde{W}}{2}\right)}
$$

when rearranging Eqn. 4.15a) and

$$
\bar{\gamma}_{\lambda}=\frac{\left(\tilde{\kappa}^{2}+\tilde{\lambda}^{2}\right) \tanh \left(\frac{\tilde{\lambda} \tilde{W}}{2}\right)}{\tilde{\lambda} \tanh \left(\frac{\tilde{\lambda} \tilde{W}}{2}\right)+\tilde{\kappa} \tan \left(\frac{\tilde{\kappa} \tilde{W}}{2}\right)} .
$$

when rearranging Eqn. 4.15b). To obtain these relations, we have made use of Eqns. A.3 and A.4 in the appendix.

Now we proceed to determine solutions for topological evanescent edge states, i.e. when $|\tilde{E}|<\left|\xi_{\mathrm{M}}\right|$. In the case of $\xi_{\mathrm{M}} \geq 0$, Eqns. 4.15a and 4.15b become

$$
\begin{aligned}
-\frac{\operatorname{coth}\left(\frac{\bar{\kappa} \tilde{W}}{2}\right)}{\operatorname{coth}\left(\frac{\lambda \tilde{W}}{2}\right)} & =\bar{\gamma}_{\bar{\kappa}} \bar{\gamma}_{\lambda} \\
-\frac{\tanh \left(\frac{\bar{\kappa} \tilde{W}}{2}\right)}{\tanh \left(\frac{\lambda \tilde{W}}{2}\right)} & =\bar{\gamma}_{\bar{\kappa}} \bar{\gamma}_{\lambda}
\end{aligned}
$$


where $\sigma=1$. We see that the left-hand side (LHS) of the equations is a combination that is always negative and the right-hand side (RHS) is always positive. Therefore the two sides of the equations are only equal when they are separately equal to zero. When $\bar{\gamma}_{\bar{\kappa}} \bar{\gamma}_{\lambda}=0$, we have $\tilde{E}=\xi_{\mathrm{M}}$. This is not within the gap hence there are no topological bound states between $|\tilde{E}|<\left|\xi_{\mathrm{M}}\right|$ for $\xi_{\mathrm{M}} \geq 0$, as was expected in Sec. 1.5.

For $0>\xi_{\mathrm{M}} \geq-1 / 4$, these equations are different because $\sigma=-1$ in this case. The secular equations thus become

$$
\begin{aligned}
& \frac{\operatorname{coth}\left(\frac{\bar{\kappa} \tilde{W}}{2}\right)}{\operatorname{coth}\left(\frac{\lambda \tilde{W}}{2}\right)}=\bar{\gamma}_{\bar{\kappa}} \bar{\gamma}_{\lambda} \\
& \frac{\tanh \left(\frac{\bar{\kappa} \tilde{W}}{2}\right)}{\tanh \left(\frac{\lambda \tilde{W}}{2}\right)}=\bar{\gamma}_{\bar{\kappa}} \bar{\gamma}_{\lambda}
\end{aligned}
$$

which results in a varying number of solutions. Due to the consequences of SQW QRs generally not possessing energy-reflection symmetry 1 , this may result in zero, one or two viable topological bound state solutions, dependent of the configurations of parameters $\xi_{\mathrm{M}}<0, \tilde{D}$ and $\tilde{W}$, as compared to zero and two in SLG QRs. As previously expected, we have proof that TPES can only exist in the inverted regime.

For $W \rightarrow \infty$ and $\xi_{\mathrm{M}}<0$, there exist two evanescent bound states for each $\tau$ valley within the band gap. At this limit, the energies of the edge states converge to the same energy which is determined by

$$
\bar{\gamma}_{\bar{\kappa}} \bar{\gamma}_{\lambda}=1
$$

where we have applied the limit on Eqns. 4.19a and 4.19b). The energy is thus given by

$$
E_{\mathrm{SQW}, 0}^{(\tau, \pm 1)} / E_{0}=\xi_{\mathrm{M}} \tilde{D}
$$

which agrees with the results in Ref. [7].

\footnotetext{
${ }^{1}$ Recall that this is due to the $\sigma_{0}$ term in the $H_{\mathrm{SQW}, 0}^{(\tau)}$ [see Eqn. $2.17 \mathrm{a}$ ] ] which is present when $\tilde{D} \in 0$. The $\sigma_{0}$ terms is what we call a vector potential and couples to the charge (unlike $\sigma_{2}$ which couples to the mass) of the particle thus treating electrons and holes oppositely, hence causing an asymmetry in their dispersions.
} 
Now we explore the effects of changing certain parameters, such as width, and how this affects the secular equation solutions. First we turn our attention to the simpler energy-reflection symmetric case of $\tilde{D}=0$ (thus $\left.E_{\mathrm{SQW}, 0}^{(\tau, n)}=-E_{\mathrm{SQW}, 0}^{(\tau,-n)}\right)$. We plot $\tilde{E} q_{0} W$ as a function of $\xi_{\mathrm{M}} q_{0} W$ for the three lowest-subbands in Fig. (4.1). The parametrisation is chosen such that our axes are analogous to those for the SLG Fig. (3.1): $\xi_{\mathrm{M}} q_{0} W \equiv V_{0} /(\hbar v / W)$. Unlike SLG, there is no universal energy curve because the curves change for different $\tilde{W}$ values. It becomes apparent that as $\tilde{W} \rightarrow \infty$, the curves approach those of SLG. The underlying reason for this is due to the quan$\operatorname{tity} \tanh (\tilde{\lambda} \tilde{W} / 2) \rightarrow 1$, which causes the secular equations [Eqns. 4.15a) and (4.15b] to resemble those of SLG [Eqns. 3.11a and (3.11b), respectively].

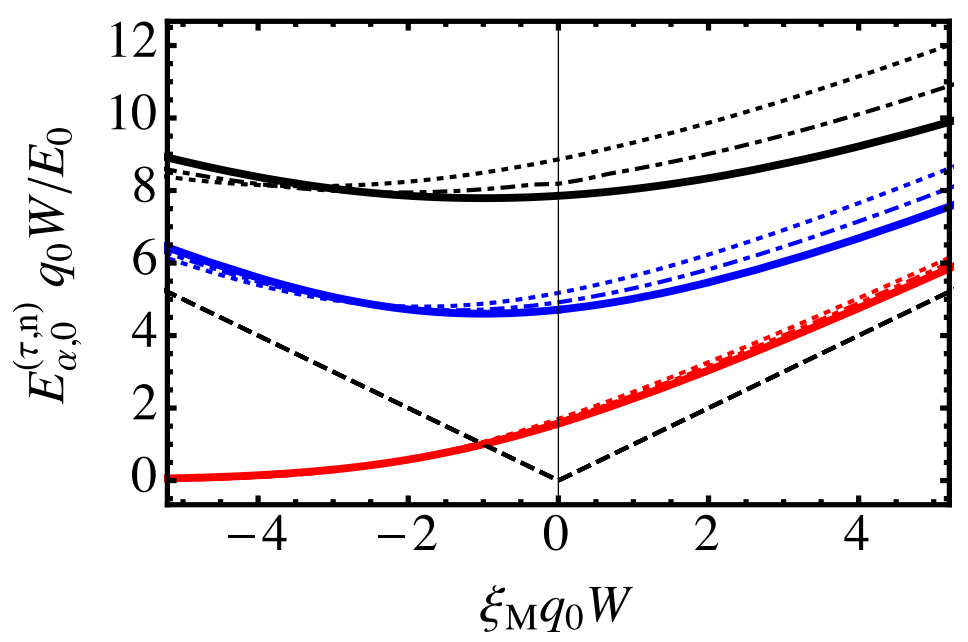

Figure 4.1: Three $\tilde{D}=0$ lowest-subbands energies $\tilde{E} q_{0} W$ versus $\xi_{\mathrm{M}} q_{0} W$ at different $q_{0} W$ values for $S Q W$ (discontinuous) and $S L G$ (thick-solid). The different subbands are given by $n=1$ (red), 2 (blue) and 3 (black). The width values are given by $q_{0} W=26.6$ (dotted) and 53.1 (dot-dashed). The dashed-black line represents the gap-size: $E_{\alpha, 0}^{(\tau, n)} / E_{0}=\left|\xi_{\mathrm{M}}\right|$.

In Fig. (4.1), we also observe that when $\xi_{\mathrm{M}} q_{0} W \lesssim-1$, the lowest-subband becomes topologically protected allowing for the existence of evanescent edge states with energies $|\tilde{E}|<\left|\xi_{\mathrm{M}}\right|$. This is an approximate limit since varying the width changes the point at which the system becomes topological. However, this change is very small for a wide range of $\tilde{W}$ values (particularly for large $\tilde{W})$. Thus, the limit is reminiscent to that of the SLG case [see Eqn. 3.13] ] which further emphasises the similarities between the SQW and SLG for large $\tilde{W}$. Furthermore, in the limit of $\xi_{\mathrm{M}} q_{0} W \rightarrow-\infty$ we see that $\tilde{E} q_{0} W \rightarrow 0$ for 
the lowest-subband. This is distinctly different from the behaviour of higher subbands which have $\tilde{E} q_{0} W \rightarrow \infty$. All these defining features draw attention to the topological nature of this subband.

For a negative non-zero $\tilde{D} 2$, energy-reflection symmetry no longer holds. We plot the energy as a function of gap size for different $\pm n$ subbands in Fig. 4.2. Contrary to the $\tilde{D}=0$ case, the lowest-subbands now become evanescent at two different values of $\xi_{\mathrm{M}} q_{0} W$ thus resulting in varying numbers of states $(0,1$ or 2$)$ within the gap at a given $\xi_{\mathrm{M}} q_{0} W$ configuration. The system is in its topological phase once the first evanescent TPES state forms [e.g. in Fig. (4.2) where $\left.\xi_{\mathrm{M}} q_{0} W \approx-0.5\right]$.

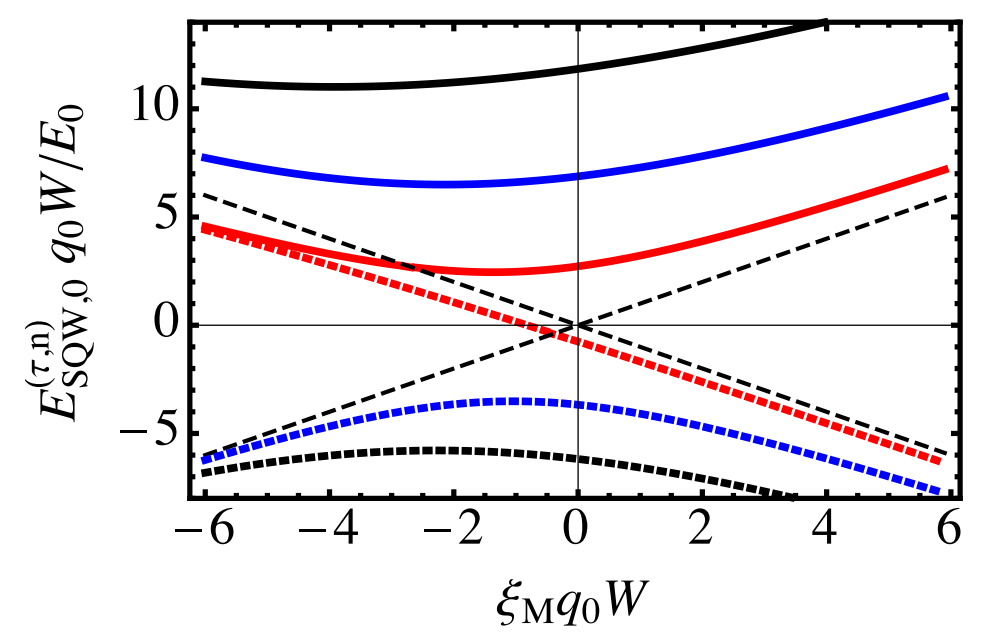

Figure 4.2: Energy curves of the lowest-three $\pm n$ (solid/dotted) subbands where we plot $E q_{0} W / E_{0}$ versus $\xi_{\mathrm{M}} q_{0} W$ for a $S Q W$ QR when $D /|B|=$ -0.746 . The subbands are given by $n= \pm 1$ (red), \pm 2 (blue) and \pm 3 (black). The dashed-black line represents the gap-size: $E_{\mathrm{SQW}, 0}^{(\tau, n)} / E_{0}= \pm\left|\xi_{\mathrm{M}}\right|$. The width was held constant at $q_{0} W=26.6$.

Unlike the $\tilde{D}=0$ case, the $n= \pm 1$ lowest-subbands do not tend to zero in the limit of $\xi_{\mathrm{M}} q_{0} W \rightarrow-\infty$. However, they still behave visibly different from the higher subbands because they converge to the same values while the higher subbands go to $\pm \infty$, respectively. This means that the energy difference of the lowest-subband goes to zero in the deeply inverted regime which will be important in Chapter 6. The energy, to which the lowest-subbands converge, is given by the value $E_{\mathrm{SQW}, 0}^{(\tau, n)} / E_{0} \rightarrow \xi_{\mathrm{M}} \tilde{D}$, as shown in Eqn. 4.21. In contrast, the higher subbands go as $E_{\mathrm{SQW}, 0}^{(\tau, n)} / E_{0} \rightarrow \infty$ as $\xi_{\mathrm{M}} q_{0} W \rightarrow-\infty$. This once

\footnotetext{
${ }^{2} \mathrm{As}$ in the case of HgTe quantum wells.
} 
again signifies the difference between the the topologically protected state (when $\left|E_{\mathrm{SQW}, 0}^{(\tau, n)}\right|<\left|\xi_{\mathrm{M}}\right|$ ) versus the higher subbands.

The shapes of higher subbands have also changed slightly: for $n<-1$, the subbands are more "bunched" together while the $n>1$ subbands are more spread out. This effect would be reversed for a positive value of $\tilde{D}$. As for the width dependence of the energies, although not shown in the figure, we see the same effect as in the $\tilde{D}=0$ case where for large values of $q_{0} W$ the energy solutions appear at similar values for a given gap size. Although not proven, we strongly suspect that for $q_{0} W \rightarrow \infty$ the curves would approach the curves produced by a modified SLG case [i.e. with the addition of $\tilde{D} \sigma_{0} \partial_{r}^{2}$ to Eqn. (3.3)].

As mentioned before, in Fig. (4.2), there are a varying number of TPESs for different values of $\xi_{\mathrm{M}}$ and $q_{0} W$ for a non-zero value of $\tilde{D}$. A detailed discussion on this topic is presented in the next section where we determine the values of $\xi_{\mathrm{M}}$ and $q_{0} W$ where TPESs disappear.

\subsubsection{Transition from topological regime to normal}

As mentioned in the previous sections, topological bound states (i.e. TPES) are only present at certain values of $\xi_{\mathrm{M}}, \tilde{D}$ and $\tilde{W}$, as seen in Figs. 4.1 and (4.2). We also know from Sec. (4.1.1) that there are none when $\xi_{\mathrm{M}} \geq 0$. However, for $\xi_{\mathrm{M}}<0$, the situation still varies depending on the magnitudes of $\left|\xi_{\mathrm{M}}\right|, \tilde{W}$ and $\tilde{D}$. When $\tilde{D}=0$, we either have zero or two topologically protected edge states at a given $\xi_{\mathrm{M}}, \tilde{W}$ and $\tau$ value due to energy-reflection symmetry. However, when $\tilde{D} \in 0$, we can have $\{0,1,2\}$ edge states at a given $\xi_{\mathrm{M}}, \tilde{W}$ and $\tau$ value. How these variables change the topological nature of the states and the material will now be discussed.

Although we have mostly focused our attention in previous sections on how the energies of the bound states vary with the gap size, $\xi_{\mathrm{M}}$, we will first focus on how these TPESs vary as a function of the width, $\tilde{W}$. The reason for this approach is that this knowledge is simpler to attain and will become useful for finding the variation with regards to the gap because of their intertwined relationship.

The general wavefunction [Eqn. (4.7)] can be thought of as edge states that 
are localised at $r_{i}$ and $r_{o}$ [7]. The magnitude of the coupling between these states, which depends on the confinement width $\tilde{W}$, determines the confinement energy of the overall system. For a fixed gap size $\xi_{\mathrm{M}}$ and a large width, the bound states do not interact, thus allowing them to possess the same energies, as given by Eqn. (4.21), which is below $\xi_{\mathrm{M}}$. Hence, they are TPESs. However, decreasing the width of the ring causes the coupling between the edge states, situated on opposite boundaries of the walls, to increase. This causes the magnitude of the confinement energy to increase thus eventually pushing the bound state energies out of the $|\tilde{E}|<\left|\xi_{\mathrm{M}}\right|$ region. Below a certain critical width, $\tilde{W}_{c \pm}$, the topological properties of an evanescent bound state are destroyed by the confinement effects. This process can be seen in Fig. (4.3).

To find an analytical solution for the length scale at which topological bound states become trivial, we assume $\tilde{\lambda} \quad \tilde{\bar{\kappa}}$ and $\tilde{\lambda} \tilde{W}>1[58 \mid$ such that the Eqns. 4.19a) and 4.19b become

$$
\bar{\gamma}_{\overline{\bar{\kappa}}} \bar{\gamma}_{\tilde{\lambda}}=\left\{\begin{array}{c}
\operatorname{coth}\left(\frac{\tilde{\bar{\kappa}} \tilde{W}}{2}\right) \\
\tanh \left(\frac{\tilde{\bar{\kappa}} \tilde{W}}{2}\right)
\end{array}\right.
$$

which can be rearranged to become

$$
\tilde{W}=\left\{\begin{array}{l}
\frac{2}{\tilde{\bar{\kappa}}} \operatorname{coth}^{-1}\left(\bar{\gamma}_{\bar{\kappa}} \bar{\gamma}_{\lambda}\right) \\
\frac{2}{\tilde{\tilde{\kappa}}} \tanh ^{-1}\left(\bar{\gamma}_{\bar{\kappa}} \bar{\gamma}_{\lambda}\right)
\end{array}\right.
$$

The topological bound state disappears when $|\tilde{E}| \geq\left|\xi_{\mathrm{M}}\right|$ resulting in a critical width $\tilde{W}_{c \pm}$ :

$$
\tilde{W}_{c+}=\lim _{\tilde{E} \rightarrow-\xi_{\mathrm{M}}} \frac{2}{\tilde{\bar{\kappa}}(\tilde{E})} \operatorname{coth}^{-1}\left(\bar{\gamma}_{\bar{\kappa}}(\tilde{E}) \bar{\gamma}_{\lambda}(\tilde{E})\right)
$$

and

$$
\tilde{W}_{c-}=\lim _{\tilde{E} \rightarrow \xi_{\mathrm{M}}} \frac{2}{\tilde{\bar{\kappa}}(\tilde{E})} \tanh ^{-1}\left(\bar{\gamma}_{\bar{\kappa}}(\tilde{E}) \bar{\gamma}_{\lambda}(\tilde{E})\right)
$$




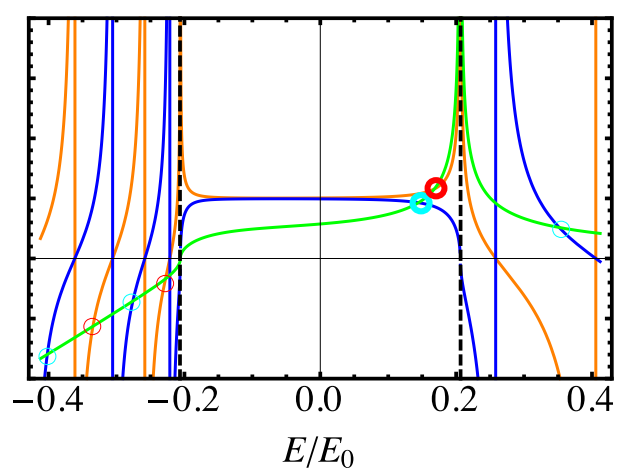

(a) $\tilde{W}>\tilde{W}_{c+}$

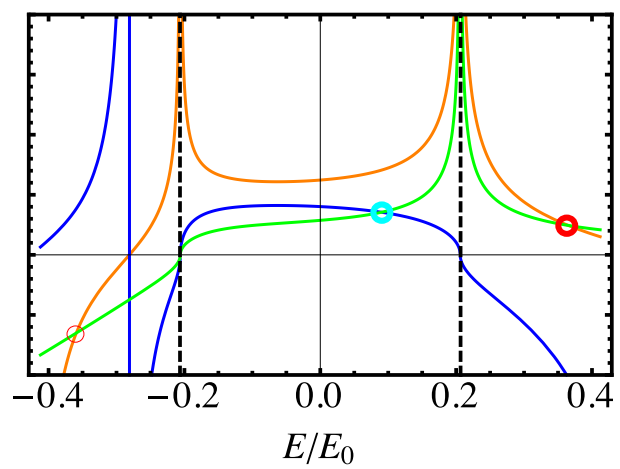

(c) $\tilde{W}_{c-}<\tilde{W}<\tilde{W}_{c+}$

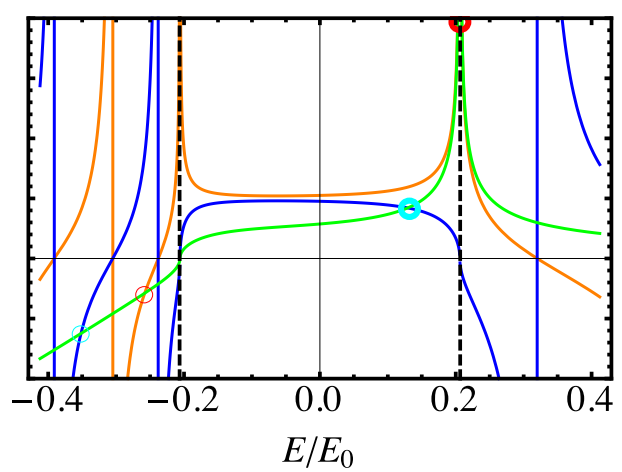

(b) $\tilde{W}=\tilde{W}_{c+}$

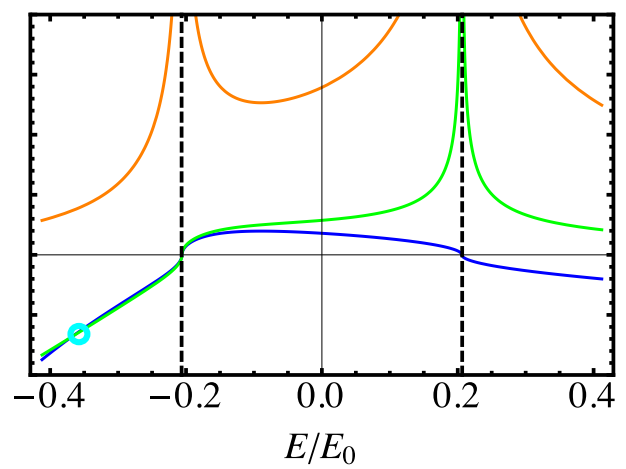

(d) $\tilde{W}<\tilde{W}_{c-}$

Figure 4.3: Transition of the bound states (red and blue circles) between different limits of $\tilde{W}$ with respect to $\tilde{W}_{c \pm}$. We plot the RHS (orange and blue respectively) and LHS (green for both) of Eqns. (4.15a) and (4.15b) and note the points of overlaps (i.e. bound states). The thick-red (thick-blue) bound state moves from within the gap $\left( \pm\left|\xi_{\mathrm{M}}\right|\right.$ indicated by vertical dashed black lines) to outside the gap after $\tilde{W}$ becomes smaller than the critical width $\tilde{W}_{c+}\left(\tilde{W}_{c-}\right)$. The parameters are as follows: $\xi_{\mathrm{M}}=-0.207, \tilde{D}=-0.746$ and $q_{0} W=20,13.4,8,2.3[(\mathbf{a})-(\mathbf{d})]$.

Note that the limits were chosen based of Fig. (4.3) where the type (a) and (b) bound states always transition out of the gap at $\mp \xi_{\mathrm{M}}$, respectively. The critical width is found to be

$$
\tilde{W}_{c \pm}=\frac{1}{\left|\xi_{\mathrm{M}}\right|} \sqrt{\frac{1 \mp \tilde{D}}{(1 \pm \tilde{D})\left(1+2(1 \pm \tilde{D}) \xi_{\mathrm{M}}\right)}}
$$

where we have assumed $1-\tilde{D}^{2}>0$ which is generally true. Notice that the critical widths are always real for $\xi_{\mathrm{M}} \geq-1 / 4$ since $|\tilde{D}|<1$. In the special 
case of $\tilde{D}=0, \tilde{W}_{c+}=\tilde{W}_{c-}=\tilde{W}_{c}$ thus giving

$$
\tilde{W}_{c}=\frac{1}{\left|\xi_{\mathrm{M}}\right|} \sqrt{\frac{1}{1+2 \xi_{\mathrm{M}}}}
$$

where $\tilde{W}_{c}$ is a critical width below which both topological bound states disappear making the system topologically trivial.

In literature, the system parameters of $\mathrm{HgTe}$ quantum wells are generally $\hbar v=364.5 \mathrm{meV} \mathrm{nm}, B=-686 \mathrm{meV} \mathrm{nm}^{2}$ and $D=-512 \mathrm{meV} \mathrm{nm}^{2}$ [6, 7] with a variable gap magnitude. To give the reader a feeling of the length scales at which this confinement effect destroys a TPES, we calculate the critical widths for a gap parameter of $M=-40 \mathrm{meV}$ where we have $\xi_{\mathrm{M}}=-0.207$ and $\tilde{D}=-0.746$. For these values the critical widths are $\tilde{W}_{c+}=13.4(\sim 7.12 \mathrm{~nm})$ and $\tilde{W}_{c-}=3.50(\sim 1.86 \mathrm{~nm})$. We notice that $\tilde{W}_{c+}>\tilde{W}_{c-}$ for $\tilde{D}<0$ and $\tilde{W}_{c+}<\tilde{W}_{c-}$ for $\tilde{D}>0$, as can be seen in Fig. $4.4 \mathrm{~b}$. Additionally, one must note that the approximation $\tilde{\lambda}>\tilde{\bar{\kappa}}$ and $\tilde{\lambda} \tilde{W} \quad 1$ can be slightly inaccurate for the $\min \left\{\tilde{W}_{c+}, \tilde{W}_{c-}\right\}$ and thus lead to a minor shift from the actual value.

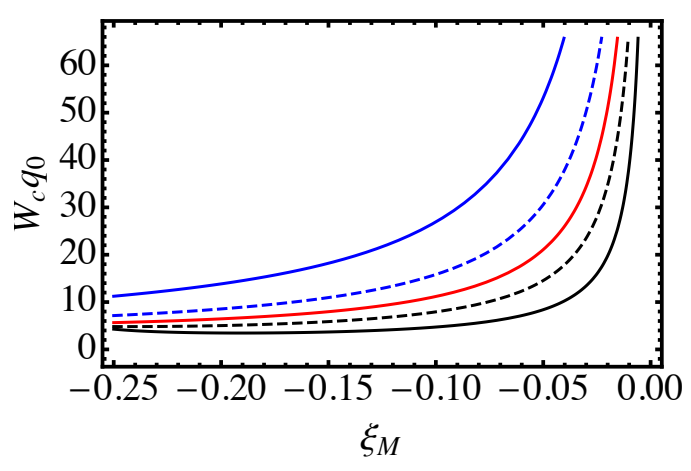

(a) $\tilde{W}_{c \pm}$ versus $\xi_{\mathrm{M}}$

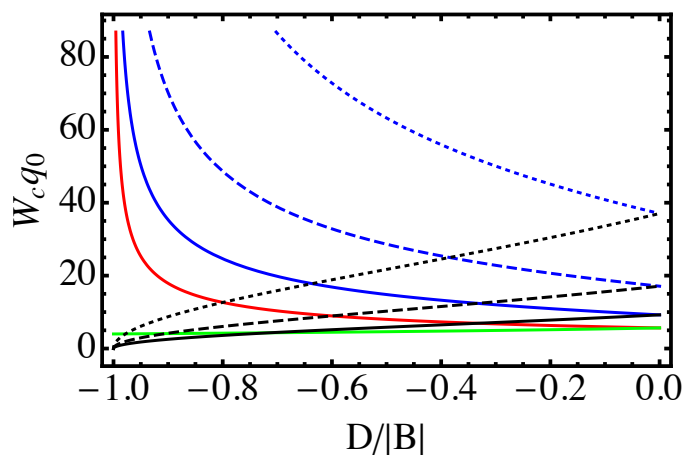

(b) $\tilde{W}_{c \pm}$ versus $\tilde{D}$

Figure 4.4: Critical width as a function of $\xi_{\mathrm{M}}$ and $\tilde{D}$. (a) The different values of $\tilde{D}$ are $\mathbf{\pm} 0.746$ (solid blue/black for $\tilde{W}_{c+}$ or vice versa for $\tilde{W}_{c-}$ ), $\mathbf{\pm} 0.373$ (dashed blue/black) and 0 (red). (b) The different values of $\xi_{\mathrm{M}}$ are $-1 / 4$ (red for $\tilde{W}_{c+}$ and green for $\tilde{W}_{c-}$ ), $-1 / 8$ (solid blue/black), $-1 / 16$ (dashed blue/black) and $-1 / 32$ (dotted blue/black).

Curiously, varying the magnitude of $\left|\xi_{\mathrm{M}}\right|$ causes similar effects as varying the width. When we are in the deeply inverted region, there are usually two TPES within the gap for a wide range of width values [see Fig. 4.4a]. However, as we decrease the magnitude of the inverted band gap, i.e. $\left|\xi_{M}\right| \rightarrow$ 0, we see states that were TPES become trivial, as seen in Fig. 4.2. To find 
the gap values at which TPES disappear, Eqn. 4.26 must be inverted for the critical values $\xi_{\mathrm{Mc} \pm}$.

The change of $\xi_{\mathrm{Mc}} q_{0} W$ as a function of $q_{0} W$ can be seen in Fig. (4.5). For a large limit of $\tilde{W} \quad 1$ [and thus $\xi_{\mathrm{Mc} \pm} 1$ to satisfy Eqn. 4.26)] we find, via a Taylor expansion of Eqn. 4.26), that the system has topological bound states when

$$
\xi_{\mathrm{Mc} \pm} q_{0} W \lesssim-\sqrt{\frac{1 \mp \tilde{D}}{1 \pm \tilde{D}}}+\mathrm{O}\left(\xi_{\mathrm{Mc} \pm}\right)
$$

For $\tilde{D}=0$, we have $\xi_{\mathrm{Mc}+}=\xi_{\mathrm{Mc}-}=\xi_{\mathrm{Mc}}$ and the limit reduces to

$$
\xi_{\mathrm{Mc}} q_{0} W \lesssim-1
$$

which is reminiscent of the SLG limit [see Eqn. (3.13)], as was also explored in Sec. 3.1.1). This value is seen to be an accurate description at large values of $q_{0} W(\gtrsim 10)$ in Fig. 4.5). Furthermore, in Fig. (4.1) we see support for this limit since for the larger width $\left(q_{0} W=100\right)$ the limit becomes more accurate.

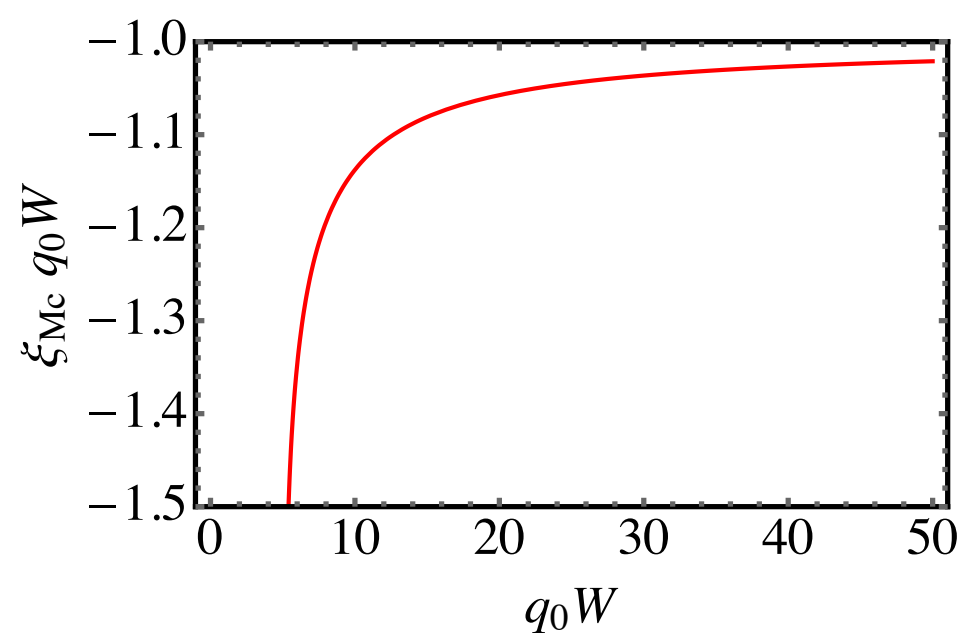

Figure 4.5: Critical $\xi_{\mathrm{Mc}} q_{0} W$ as a function of $q_{0} W$. As $q_{0} W \rightarrow \infty$, $\xi_{\mathrm{Mc}} q_{0} W \rightarrow 1$. This limit is reminiscent of the case in $S L G$. $\tilde{D}$ was held at 0 .

In the case of a non-zero $\tilde{D}=-0.746$ as in Fig. 4.2 , we have $\xi_{\mathrm{Mc} \pm} q_{0} W=$ $\{2.62,0.381\}$ which corresponds well to the transition points between topologically protected and trivial edge states. This is accurate since we have a 
fairly large $\tilde{W}=26.6$ in Fig. 4.2 .

Generally, TPES are present in un-confined SQW whenever $\xi_{\mathrm{M}}<0[2]$. However, we have shown that when SQW become ring-confined, TPES are only present if $W>W_{\mathrm{c} \pm}$ and $\xi_{\mathrm{M}}<\xi_{\mathrm{Mc} \pm}$. Overall we have found that quantum confinement of SQW QRs causes some of the TPES that were present in the initial un-confined SQW to disappear, as in the SLG QR case.

We have now determined and explored the critical limits at which TPESs appear and have gained a comprehensive understanding of how the secular energy solutions vary for different system parameters. This section will be crucial for our understanding of the $\tau$ valley dependence for the subband energy dispersions which will be discussed in Sec. (4.2.2). It will also aid us in comprehending results in Chapter 6 where we shall discover connections between the Berry phase, arising due to quantum-confinement, and the critical limits at which TPES dissappear. We now move on to determine the energy eigenstates of $H_{\mathrm{SQW}, 0}^{(\tau)}$.

\subsubsection{Eigenstates of SQW}

The boundary equation matrix determined by the boundary conditions

$$
\chi_{\mathrm{SQW}}^{(\tau, n)}\left(r_{i / o}\right)=0
$$

can be shown to be

$$
\left(\begin{array}{cccc}
e^{\frac{i \tilde{W} \tilde{\kappa}}{2}} & e^{-\frac{i \tilde{W} \tilde{\kappa}}{2}} & \bar{\gamma}_{\lambda} e^{-\frac{\tilde{W} \tilde{\lambda}}{2}} & \bar{\gamma}_{\lambda} e^{\frac{\tilde{W} \tilde{\lambda}}{2}} \\
e^{-\frac{i \tilde{W} \tilde{\kappa}}{2}} & e^{\frac{i \tilde{W} \tilde{K}}{2}} & \bar{\gamma}_{\lambda} e^{\frac{\tilde{W} \tilde{\lambda}}{2}} & \bar{\gamma}_{\lambda} e^{-\frac{\tilde{W} \tilde{\lambda}}{2}} \\
\tau \sigma \gamma_{\kappa} e^{\frac{i \tilde{W} \tilde{\kappa}}{2}} & -\tau \sigma \gamma_{\kappa} e^{-\frac{i \tilde{W} \tilde{\kappa}}{2}} & -i \tau e^{-\frac{\tilde{W} \tilde{\lambda}}{2}} & i \tau e^{\frac{\tilde{W} \tilde{\lambda}}{2}} \\
\tau \sigma \gamma_{\kappa} e^{-\frac{i \tilde{W} \tilde{\kappa}}{2}} & -\tau \sigma \gamma_{\kappa} e^{\frac{i \tilde{W} \tilde{\kappa}}{2}} & -i \tau e^{\frac{\tilde{W} \tilde{\lambda}}{2}} & i \tau e^{-\frac{\tilde{W} \tilde{\lambda}}{2}}
\end{array}\right)\left(\begin{array}{c}
c_{1 \kappa}^{(\tau, n)} \\
c_{2 \kappa}^{(\tau, n)} \\
c_{1 \lambda}^{(\tau, n)} \\
c_{2 \lambda}^{(\tau, n)}
\end{array}\right)=\left(\begin{array}{l}
0 \\
0 \\
0 \\
0
\end{array}\right)
$$

Using the first three equations in the matrix, we can determine the general values of $c_{1 \kappa}^{(\tau, n)}, c_{2 \kappa}^{(\tau, n)}$ and $c_{1 \lambda}^{(\tau, n)}$ in terms of $c_{2 \lambda}^{(\tau, n)}$ which allows us to find the general form of the wavefunction. This derivation can be seen in Appendix A.3.

However, it is advantageous for us to consider a simplified version of the wavefunction by separating it into two forms that correspond to type (a) states [i.e. energies described by Eqn. (4.15a)] and type (b) states [i.e. energies de- 
scribed by Eqn. 4.15b]]. This can be obtained by substituting Eqns. 4.16) and (4.17) into the $\gamma_{\kappa}$ and $\bar{\gamma}_{\lambda}$ components of Eqn. (4.30) ${ }^{3}$. When we then solve for the wavefunction we find

$$
\chi_{\mathrm{SQW}, 1}^{(\tau, n)}(\tilde{r})=\tilde{c}_{1} \sqrt{ }_{\overline{q_{0}}}\left(\begin{array}{c}
\frac{\cosh (\tilde{\lambda}(\tilde{r}-\tilde{R}))}{\cosh \left(\frac{\hat{\lambda} \tilde{W}}{2}\right)}-\frac{\cos (\tilde{\kappa}(\tilde{r}-\tilde{R}))}{\cos \left(\frac{\tilde{\kappa} \tilde{W}}{2}\right)} \\
\frac{i \tau\left(\tilde{\kappa}^{2}+\tilde{\lambda}^{2}\right)}{\tilde{\lambda} \operatorname{coth}\left(\frac{\tilde{\lambda} \tilde{W}}{2}\right)-\tilde{\kappa} \cot \left(\frac{\tilde{\kappa} \tilde{W}}{2}\right)}\left(\frac{\sinh (\tilde{\lambda}(\tilde{r}-\tilde{R}))}{\sinh \left(\frac{\tilde{\lambda} \tilde{W}}{2}\right)}-\frac{\sin (\tilde{\kappa}(\tilde{r}-\tilde{R}))}{\sin \left(\frac{\kappa}{2} \tilde{W}\right)}\right)
\end{array}\right)
$$

for type (a) states [using Eqn. (4.16)], and

$$
\chi_{\mathrm{SQW}, 2}^{(\tau, n)}(\tilde{r})=\tilde{c}_{2} \sqrt{ }_{\bar{q}}\left(\begin{array}{c}
\frac{\sinh (\tilde{\lambda}(\tilde{r}-\tilde{R}))}{\sinh \left(\frac{\hat{\lambda} \tilde{W}}{2}\right)}-\frac{\sin (\tilde{\kappa}(\tilde{r}-\tilde{R}))}{\sin \left(\frac{\tilde{\kappa} \tilde{W}}{2}\right)} \\
\frac{i \tau\left(\tilde{\kappa}^{2}+\tilde{\lambda}^{2}\right)}{\tilde{\lambda} \tanh \left(\frac{\tilde{\lambda} \tilde{W}}{2}\right)+\tilde{\kappa} \tan \left(\frac{\tilde{K} \tilde{W}}{2}\right)}\left(\frac{\cosh (\tilde{\lambda}(\tilde{r}-\tilde{R}))}{\cosh \left(\frac{\tilde{\lambda} \tilde{W}}{2}\right)}-\frac{\cos (\tilde{\kappa}(\tilde{r}-\tilde{R}))}{\cos \left(\frac{\tilde{\kappa} \tilde{W}}{2}\right)}\right)
\end{array}\right)
$$

for type (b) states [using Eqn. (4.17)], where $\tilde{c}_{1}$ and $\tilde{c}_{2}$ are normalisation constants. These wavefunctions are equivalent to those derived by B. Zhou et. al. 7$]^{4}$.

Remembering that we are working in a ring geometry, the actual wavefunctions are given by $\phi_{\mathrm{SQW}, 1}^{(\tau, n)}=\chi_{\mathrm{SQW}, 1}^{(\tau, n)} / \sqrt{2 \pi r}$ and $\phi_{\mathrm{SQW}, 2}^{(\tau, n)}=\chi_{\mathrm{SQW}, 2}^{(\tau, n)} / \sqrt{2 \pi r}$. Both $\phi_{\mathrm{SQW}, 1}^{(\tau, n)}(\tilde{r})$ and $\phi_{\mathrm{SQW}, 2}^{(\tau, n)}(\tilde{r})$ can be separated into two components which are associated with the Dirac part and the wing (decaying) part of the wavefunction, respectively:

$$
\phi_{\mathrm{SQW}, \eta}^{(\tau, n)}(\tilde{r})=\phi_{\mathrm{SQW}, \eta, D}^{(\tau, n)}(\tilde{r})+\phi_{\mathrm{SQW}, \eta, W}^{(\tau, n)}(\tilde{r})
$$

where $\eta \equiv\{1,2\}$. For $\phi_{\mathrm{SQW}, 1}^{(\tau, n)}(\tilde{r})$, we have

$$
\phi_{\mathrm{SQW}, 1, D}^{(\tau, n)}(\tilde{r})=-\sqrt{\overline{2 \pi \tilde{r}}} \frac{\tilde{c}_{1}}{\cos \left(\frac{\tilde{\kappa} \tilde{W}}{2}\right)}\left(\begin{array}{c}
\cos (\tilde{\kappa}(\tilde{r}-\tilde{R})) \\
i \tau \sigma \gamma_{\kappa} \sin (\tilde{\kappa}(\tilde{r}-\tilde{R}))
\end{array}\right)
$$

\footnotetext{
${ }^{3}$ For type (a) states the $\bar{\gamma}_{\lambda}$ is found as a function of $\gamma_{\kappa}$ using the secular equation [Eqn. 4.15a]]. Then all the $\gamma_{\kappa}$ components are substituted using Eqn. (4.16). For type (b) states, the procedure is reversed where $\gamma_{\kappa}$ is written in terms of $\bar{\gamma}_{\lambda}$ and then substituted using Eqn. (4.17).

${ }^{4}$ Minor differences arise due to a choice of basis: we work using the $x$ axis orientation of $r$ instead of their $y$ axis orientation. Hence there is no effect on the physical results.
} 
and

$$
\phi_{\mathrm{SQW}, 1, W}^{(\tau, n)}(\tilde{r})=\frac{{ }^{\tilde{c}_{1}}}{\overline{2 \pi \tilde{r}}} \frac{q_{0}}{\cosh \left(\frac{\tilde{\lambda} \tilde{W}}{2}\right)}\left(\begin{array}{c}
\cosh (\tilde{\lambda}(\tilde{r}-\tilde{R})) \\
i \tau \frac{1}{\bar{\gamma}_{\lambda}} \sinh (\tilde{\lambda}(\tilde{r}-\tilde{R}))
\end{array}\right)
$$

where $\tilde{c}_{1}$ is given by

$$
\begin{gathered}
\tilde{c}_{1}^{-2}=\frac{\tilde{W}}{2}\left[\frac{1+\gamma_{\kappa}^{2}}{\cos ^{2}\left(\frac{\kappa W}{2}\right)}+\frac{1-\frac{1}{\bar{\gamma}_{\lambda}^{2}}}{\cosh ^{2}\left(\frac{\lambda W}{2}\right)}\right]-\left[\frac{4\left(\frac{\sigma \gamma_{\kappa}}{\bar{\gamma}_{\lambda}} \tilde{\lambda}+\tilde{\kappa}\right)}{\tilde{\kappa}^{2}+\tilde{\lambda}^{2}}+\frac{\gamma_{\kappa}^{2}-1}{\tilde{\kappa}}\right] \tan \left(\frac{\tilde{\kappa} \tilde{W}}{2}\right) \\
+\left[\frac{4\left(\frac{\sigma \gamma_{\kappa}}{\bar{\gamma}_{\lambda}} \tilde{\kappa}-\tilde{\lambda}\right)}{\tilde{\kappa}^{2}+\tilde{\lambda}^{2}}+\frac{\frac{1}{\overline{\bar{\gamma}}_{\lambda}^{2}}+1}{\tilde{\lambda}}\right] \tanh \left(\frac{\tilde{\lambda} \tilde{W}}{2}\right)
\end{gathered}
$$

and for $\phi_{\mathrm{SQW}, 2}^{(\tau, n)}(r)$, we have

$$
\phi_{\mathrm{SQW}, 2, D}^{(\tau, n)}(\tilde{r})=-\sqrt{\frac{\tilde{c}_{2}}{2 \pi \tilde{r}}} \frac{q_{0}}{\sin \left(\frac{\tilde{\kappa} \tilde{W}}{2}\right)}\left(\begin{array}{c}
\sin (\tilde{\kappa}(\tilde{r}-\tilde{R})) \\
-i \tau \sigma \gamma_{\kappa} \cos (\tilde{\kappa}(\tilde{r}-\tilde{R}))
\end{array}\right)
$$

and

$$
\phi_{\mathrm{SQW}, 2, W}^{(\tau, n)}(\tilde{r})=\sqrt{\frac{\tilde{c}_{2}}{2 \pi \tilde{r}}} \frac{q_{0}}{\sinh \left(\frac{\tilde{\lambda} \tilde{W}}{2}\right)}\left(\begin{array}{c}
\sinh (\tilde{\lambda}(\tilde{r}-\tilde{R})) \\
i \tau \frac{1}{\overline{\bar{\gamma}_{\lambda}}} \cosh (\tilde{\lambda}(\tilde{r}-\tilde{R}))
\end{array}\right)
$$

where $\tilde{c}_{2}$ is given by

$$
\begin{gathered}
\tilde{c}_{2}^{-2}=\frac{\tilde{W}}{2}\left[\frac{1+\gamma_{\kappa}^{2}}{\sin ^{2}\left(\frac{\kappa W}{2}\right)}-\frac{1-\frac{1}{\bar{\gamma}_{\lambda}^{2}}}{\sinh ^{2}\left(\frac{\lambda W}{2}\right)}\right]+\left[\frac{4\left(\frac{\sigma \gamma_{\kappa}}{\bar{\gamma}_{\lambda}} \tilde{\lambda}+\tilde{\kappa}\right)}{\tilde{\kappa}^{2}+\tilde{\lambda}^{2}}+\frac{\gamma_{\kappa}^{2}-1}{\tilde{\kappa}}\right] \cot \left(\frac{\tilde{\kappa} \tilde{W}}{2}\right) \\
+\left[\frac{4\left(\frac{\sigma \gamma_{\kappa}}{\bar{\gamma}_{\lambda}} \tilde{\kappa}-\tilde{\lambda}\right)}{\tilde{\kappa}^{2}+\tilde{\lambda}^{2}}+\frac{\frac{1}{\overline{\bar{\gamma}}_{\lambda}^{2}}+1}{\tilde{\lambda}}\right] \operatorname{coth}\left(\frac{\tilde{\lambda} \tilde{W}}{2}\right)
\end{gathered}
$$

Upon seeing the separated wavefunctions, it is obvious that Eqns. (4.34) and (4.37) have identical forms (up to a constant factor) to the wavefunctions obtained by the 1D Dirac equation, given by Eqns. (3.16a) and (3.16b). This exemplifies the Dirac nature of SQWs with however the noticeable addition of the wing states which play a particularly observable role close to the edges of the ring [7]. The forms of the evanescent eigenstates, when $|\tilde{E}|<\left|\xi_{\mathrm{M}}\right|$, can be seen in Appendix A.4.

We have now obtained the forms of the eigenstates and energies of $H_{\mathrm{SQW}, 0}^{(\tau)}$. 
In the next section, we use these results to change the basis of $H_{\mathrm{SQW}, 1}^{(\tau)}$ using the eigenstates of $H_{\mathrm{SQW}, 0}^{(\tau)}$. This will eventually allow us to find the azimuthal (i.e. $l$ ) dependence of the subband energy dispersions.

\subsection{Finding the azimuthal dependence}

The Hamiltonian associated with the azimuthal motion, as given in Eqn. (2.17b), can be reworked to become

$$
\begin{aligned}
H_{\mathrm{SQW}, 1}^{(\tau)} & =\hbar v \frac{l}{r} \sigma_{2}-\frac{l^{2}}{r^{2}} B \sigma_{3}-D \frac{l^{2}}{r^{2}} \sigma_{0} \\
& =E_{0}\left(\frac{l}{\tilde{r}} \sigma_{2}+\frac{l^{2}}{\tilde{r}^{2}} \sigma_{3}-\tilde{D} \frac{l^{2}}{\tilde{r}^{2}} \sigma_{0}\right)
\end{aligned}
$$

where we have made use of our parametrisation definitions. This Hamiltonian determines the total subband energy dispersion and transport properties of the material due to the inclusion of the azimuthal motion of particles in the ring.

The only symmetry present in the $\mathrm{H}_{\mathrm{SQW}, 1}^{(\tau)}$ is given by

$$
\mathbf{h}_{\mathrm{SQW}, 2}^{(\tau, n)}\left|H_{\mathrm{SQW}, 1}^{(\tau)}\right| \phi_{\mathrm{SQW}, 1}^{\left(\tau, n^{\prime}\right)} \mathbf{i}=\mathbf{h} \phi_{\mathrm{SQW}, 1}^{(\tau, n)}\left|H_{\mathrm{SQW}, 1}^{(\tau)}\right| \phi_{\mathrm{SQW}, 2}^{\left(\tau, n^{\prime}\right)} \mathbf{i}^{\dagger}
$$

which is due to the Hermitian nature of the Hamiltonian. Unfortunately, since energy-reflection symmetry does not generally hold, there is no symmetry in the diagonal terms such as in the SLG and TMD cases that would simplify our calculations. Thus we have to apply the general change of basis to $H_{\mathrm{SQW}, 1}^{(\tau)}$ using the eigenstates of $H_{\mathrm{SQW}, 0}^{(\tau)}$.

We find that the matrix term $\boldsymbol{h}_{\mathrm{SQW}}^{(\tau, n)}\left|H_{\mathrm{SQW}, 1}^{(\tau)}\right| \phi_{\mathrm{SQW}}^{\left(\tau, n^{\prime}\right)} \mathbf{i}$ can be shown to be

$$
\begin{aligned}
\mathrm{h}_{\mathrm{SQW}, 1}^{(\tau, n)} \mid & H_{\mathrm{SQW}, 1}^{(\tau)} \mid \phi_{\mathrm{SQW}, 1}^{\left(\tau, n^{\prime}\right)} \mathrm{i}=\int_{R-\frac{W}{2}}^{R+\frac{W}{2}} \phi_{\mathrm{SQW}, 1}^{(\tau, n)}{ }^{\dagger} H_{\mathrm{SQW}, 1}^{(\tau)} \phi_{\mathrm{SQW}, 1}^{\left(\tau, n^{\prime}\right)} 2 \pi r d r \\
= & \tilde{c}_{1} \tilde{c}_{1}^{\prime} E_{0} \int_{\tilde{R}-\frac{\tilde{W}}{2}}^{\tilde{R}+\frac{\tilde{W}}{2}} \sum_{\alpha=1}^{2} \sum_{\beta=1}^{2} \frac{\tau_{\alpha} \tau_{\beta}^{\prime}}{\cos \left(\frac{\lambda_{\alpha} \tilde{W}}{2}\right) \cos \left(\frac{\lambda_{\beta}^{\prime} \tilde{W}}{2}\right)} \times \\
& {\left[\frac { l } { \tilde { r } } \tau \left(\frac{\gamma_{\beta}^{\prime}}{\sqrt{-\tau_{\beta}^{\prime}}} \cos \left(\lambda_{\alpha}(\tilde{r}-\tilde{R})\right) \sin \left(\lambda_{\beta}^{\prime}(\tilde{r}-\tilde{R})\right)\right.\right.}
\end{aligned}
$$




$$
\begin{gathered}
\left.+\frac{\gamma_{\alpha}}{-\tau_{\alpha}} \cos \left(\lambda_{\beta}^{\prime}(\tilde{r}-\tilde{R})\right) \sin \left(\lambda_{\alpha}(\tilde{r}-\tilde{R})\right)\right) \\
+\frac{l^{2}}{\tilde{r}^{2}}\left(\cos \left(\lambda_{\alpha}(\tilde{r}-\tilde{R})\right) \cos \left(\lambda_{\beta}^{\prime}(\tilde{r}-\tilde{R})\right)\right. \\
\left.-\frac{\gamma_{\alpha}}{-\tau_{\alpha}} \frac{\gamma_{\beta}^{\prime}}{\sqrt{-\tau_{\beta}^{\prime}}} \sin \left(\lambda_{\beta}^{\prime}(\tilde{r}-\tilde{R})\right) \sin \left(\lambda_{\alpha}(\tilde{r}-\tilde{R})\right)\right) \\
-\tilde{D} \frac{l^{2}}{\tilde{r}^{2}}\left(\cos \left(\lambda_{\alpha}(\tilde{r}-\tilde{R})\right) \cos \left(\lambda_{\beta}^{\prime}(\tilde{r}-\tilde{R})\right)\right. \\
\left.\left.+\frac{\sqrt{\frac{\gamma_{\alpha}}{-\tau_{\alpha}}}}{\sqrt{-\tau_{\beta}^{\prime}}} \sin \left(\lambda_{\beta}^{\prime}(\tilde{r}-\tilde{R})\right) \sin \left(\lambda_{\alpha}(\tilde{r}-\tilde{R})\right)\right)\right] d \tilde{r}
\end{gathered}
$$

where $\tau_{1,2}= \pm 1, \lambda_{1}=i \tilde{\lambda}, \lambda_{2}=\tilde{\kappa}, \gamma_{1}=1 / \bar{\gamma}_{\lambda}$ and $\gamma_{2}=\sigma \gamma_{\kappa}$. Note that here $\alpha$ is used as a summation index and does not indicate the material as in the methodology. Using $\mid \phi_{\mathrm{SQW}, 2}^{(\tau, n)} \mathrm{i}$, we have

$$
\begin{aligned}
& \mathbf{h} \phi_{\mathrm{SQW}, 2}^{(\tau, n)}\left|H_{\mathrm{SQW}, 1}^{(\tau)}\right| \phi_{\mathrm{SQW}, 2}^{\left(\tau, n^{\prime}\right)} \mathrm{i}= \int_{R-\frac{W}{2}}^{R+\frac{W}{2}} \phi_{S Q W, 2}^{(\tau, n)}{ }^{\dagger} H_{\mathrm{SQW}, 1}^{(\tau)} \phi_{S Q W, 2}^{\left(\tau, n^{\prime}\right)} 2 \pi r d r \\
&= \tilde{c}_{2} \tilde{c}_{2}^{\prime} E_{0} \int_{\tilde{R}-\frac{\tilde{W}}{2}}^{\tilde{R}+\frac{\tilde{W}}{2}} \sum_{\alpha=1}^{2} \sum_{\beta=1}^{2} \frac{\tau_{\alpha} \tau_{\beta}^{\prime}}{\sin \left(\frac{\lambda_{\alpha} \tilde{W}}{2}\right) \sin \left(\frac{\lambda_{\beta}^{\prime} \tilde{W}}{2}\right)} \times \\
& \quad \frac{l}{\tilde{r}} \tau\left(\tau_{\beta}^{\prime} \gamma_{\beta}^{\prime} \sqrt{-\tau_{\beta}^{\prime}} \sin \left(\lambda_{\alpha}(\tilde{r}-\tilde{R})\right) \cos \left(\lambda_{\beta}^{\prime}(\tilde{r}-\tilde{R})\right)\right. \\
&\left.+\tau_{\alpha} \gamma_{\alpha} \overline{-\tau_{\alpha}} \sin \left(\lambda_{\beta}^{\prime}(\tilde{r}-\tilde{R})\right) \cos \left(\lambda_{\alpha}(\tilde{r}-\tilde{R})\right)\right) \\
&+ \frac{l^{2}}{\tilde{r}^{2}}\left(\sin \left(\lambda_{\alpha}(\tilde{r}-\tilde{R})\right) \sin \left(\lambda_{\beta}^{\prime}(\tilde{r}-\tilde{R})\right)\right. \\
&\left.\quad-\tau_{\alpha} \tau_{\beta}^{\prime} \gamma_{\alpha} \gamma_{\beta}^{\prime} \sqrt{-\tau_{\alpha}} \sqrt{-\tau_{\beta}^{\prime}} \cos \left(\lambda_{\beta}^{\prime}(\tilde{r}-\tilde{R})\right) \cos \left(\lambda_{\alpha}(\tilde{r}-\tilde{R})\right)\right) \\
&-\tilde{D} \frac{l^{2}}{\tilde{r}^{2}}\left(\sin \left(\lambda_{\alpha}(\tilde{r}-\tilde{R})\right) \sin \left(\lambda_{\beta}^{\prime}(\tilde{r}-\tilde{R})\right)\right. \\
&\left.\left.\quad+\tau_{\alpha} \tau_{\beta}^{\prime} \gamma_{\alpha} \gamma_{\beta}^{\prime} \sqrt{-\tau_{\alpha}} \sqrt{-\tau_{\beta}^{\prime}} \cos \left(\lambda_{\beta}^{\prime}(\tilde{r}-\tilde{R})\right) \cos \left(\lambda_{\alpha}(\tilde{r}-\tilde{R})\right)\right)\right] d \tilde{r}
\end{aligned}
$$

and matrix elements that couple states $\mid \phi_{\mathrm{SQW}, 1}^{(\tau, n)} \mathrm{i}$ and $\mid \phi_{\mathrm{SQW}, 2}^{\left(\tau, n^{\prime}\right)} \mathrm{i}$ can be written 
as

$$
\begin{aligned}
& \mathrm{h}_{\mathrm{SQW}, 1}^{(\tau, n)}\left|H_{\mathrm{SQW}, 1}^{(\tau)}\right| \phi_{\mathrm{SQW}, 2}^{\left(\tau, n^{\prime}\right)} \mathrm{i}=\int_{R-\frac{W}{2}}^{R+\frac{W}{2}} \phi_{\mathrm{SQW}, 1}^{(\tau, n)}{ }^{\dagger} H_{\mathrm{SQW}, 1}^{(\tau)} \phi_{\mathrm{SQW}, 2}^{\left(\tau, n^{\prime}\right)} 2 \pi r d r \\
& =\tilde{c}_{2}^{\prime} \tilde{c}_{1} E_{0} \int_{\tilde{R}-\frac{\tilde{W}}{2}}^{\tilde{R}+\frac{\tilde{W}}{2}} \sum_{\alpha=1}^{2} \sum_{\beta=1}^{2} \frac{\tau_{\alpha} \tau_{\beta}^{\prime}}{\cos \left(\frac{\lambda_{\alpha} \tilde{W}}{2}\right) \sin \left(\frac{\lambda_{\beta}^{\prime} \tilde{W}}{2}\right)} \times \\
& {\left[\frac { l } { \tilde { r } } \tau \left(\tau_{\beta}^{\prime} \gamma_{\beta}^{\prime} \sqrt{-\tau_{\beta}^{\prime}} \cos \left(\lambda_{\alpha}(\tilde{r}-\tilde{R})\right) \cos \left(\lambda_{\beta}^{\prime}(\tilde{r}-\tilde{R})\right)\right.\right.} \\
& \left.+\sqrt{\frac{\gamma_{\alpha}}{-\tau_{\alpha}}} \sin \left(\lambda_{\beta}^{\prime}(\tilde{r}-\tilde{R})\right) \sin \left(\lambda_{\alpha}(\tilde{r}-\tilde{R})\right)\right) \\
& +\frac{l^{2}}{\tilde{r}^{2}}\left(\cos \left(\lambda_{\alpha}(\tilde{r}-\tilde{R})\right) \sin \left(\lambda_{\beta}^{\prime}(\tilde{r}-\tilde{R})\right)\right. \\
& \left.-\tau_{\beta}^{\prime} \gamma_{\alpha} \gamma_{\beta}^{\prime} \frac{\sqrt{-\tau_{\beta}^{\prime}}}{-\tau_{\alpha}} \cos \left(\lambda_{\beta}^{\prime}(\tilde{r}-\tilde{R})\right) \sin \left(\lambda_{\alpha}(\tilde{r}-\tilde{R})\right)\right) \\
& -\tilde{D} \frac{l^{2}}{\tilde{r}^{2}}\left(\cos \left(\lambda_{\alpha}(\tilde{r}-\tilde{R})\right) \sin \left(\lambda_{\beta}^{\prime}(\tilde{r}-\tilde{R})\right)\right. \\
& \left.\left.+\tau_{\beta}^{\prime} \gamma_{\alpha} \gamma_{\beta}^{\prime} \frac{\sqrt{-\tau_{\beta}^{\prime}}}{-\tau_{\alpha}} \cos \left(\lambda_{\beta}^{\prime}(\tilde{r}-\tilde{R})\right) \sin \left(\lambda_{\alpha}(\tilde{r}-\tilde{R})\right)\right)\right] d \tilde{r} .
\end{aligned}
$$

When we apply the realistic assumption that the aspect ratio $\epsilon \equiv W / R$ is small, i.e. $\epsilon \quad$, the exact solutions to these matrix elements can be found for orders of $\epsilon$ via a Taylor expansion. The solutions to these integrals, in this regime, can be found in Appendix (B) where we have tabulated the relevant integrals up to order $\epsilon^{2}$.

In the following section we explore the relative magnitudes of the matrix elements and discuss which contributions can be neglected and which must be taken account of. It is our aim to convince the reader that inter-subband interactions between $n$ and $n^{\prime} \sigma-n$ can generally be ignored thus allowing us to purely consider the $(n,-n)$ energy subbands dispersions for SQWs, as in the cases of SLG and TMD. Although this was also considered in the methodology section, we feel it prudent to show the validity of our method due to the breaking down of energy-reflection symmetry in this case. 


\subsubsection{Magnitude of matrix elements}

Using the integrals in the Appendix $(\mathrm{B})$, we can determine that, when $\epsilon \equiv$ $W / R \quad 1$, the largest contributions to the $\mathrm{H}_{\mathrm{SQW}, 1}^{(\tau)}$ are the linear $l$ terms from the $\mathrm{h}_{\mathrm{SQW}, 1}^{(\tau, n)}\left|H_{\mathrm{SQW}, 1}^{(\tau)}\right| \phi_{\mathrm{SQW}, 2}^{\left(\tau, n^{\prime}\right)} \mathrm{i}$ matrix elements. These are the only terms that are proportional to the first order of $\epsilon$. They couple type (a) and type (b) eigenstates [arising from the Eqns. (4.15a) and 4.15b)] which correspond to odd (even) and even (odd) eigenstates for positive (negative) $n$, respectively. In general, when energy-reflection symmetry holds $(\tilde{D}=0)$, the largest coupling terms are between $n$ and $-n$ states, which are always of different types, as discussed in Sec. (2.2). The reasoning for this parallels that for SLG where the difference in the magnitude of $l=0$ subband energies governs the coupling strength between the subbands. The larger the difference, the smaller the coupling. Thus in this case, the relative magnitudes of matrix elements are very similar to those of the SLG case.

However, when $\tilde{D} \sigma 0$, the strength of the coupling becomes somewhat murkier due to the fact that energies differ in magnitude for $\pm n$ subbands because of the asymmetry of the $l=0$ subband energies. Nevertheless $n$ and $-n$ states still have the strongest coupling for the lower subbands. This is because the coupling depends on the magnitude of the energy difference between the eigenstates which is relatively small for lower subbands. However for higher subbands this reasoning does not hold due to the increasing energy difference and asymmetry of the $l=0$ energies [see Fig. 44.2)]. Thus it becomes possible for higher subbands to be significantly influenced by neighbouring states since the energies become relatively small. Hence, we only fully trust the approximation described above for the lowest-subbands results in SQW.

So far we have only concentrated on the largest matrix elements of first order in $\epsilon$ for which we have established a hierarchy. However, there are also $\epsilon^{2}$ or higher order contributions, some of which cannot be neglected.

There are only odd orders of $\epsilon$ terms in $\mathbf{h}_{\mathrm{SQW}, 1}^{(\tau, n)}\left|H_{\mathrm{SQW}, 1}^{(\tau)}\right| \phi_{\mathrm{SQW}, 2}^{\left(\tau, n^{\prime}\right)} \mathrm{i}$. Thus in these matrix elements, the next order terms are 3rd and higher and can be neglected because they would be multiple orders of magnitude smaller than the $\epsilon$ terms. 
On the other hand, same type coupling terms $\left(\mathbf{h}_{\mathrm{SQW}, \eta}^{(\tau, n)}\left|H_{\mathrm{SQW}, 1}^{(\tau)}\right| \phi_{\mathrm{SQW}, \eta}^{\left(\tau, n^{\prime}\right)} \mathbf{i}\right)$ only have even orders of $\epsilon$. Once again the magnitude of individual terms depends on the difference of the energies $\Delta E^{\left(n, n^{\prime}\right)} \equiv\left|E_{\mathrm{SQW}, 0}^{(\tau, n)}\right|-\left|E_{\mathrm{SQW}, 0}^{\left(\tau, n^{\prime}\right)}\right|$ : the larger the energy difference, the smaller the contribution. Thus we can ignore $\epsilon^{2}$ and higher order contributions of inter-subband coupling terms (i.e. $n$ with $n^{\prime} 6 n$ ) since the energy differences between subbands that are separated by another subband is relatively large 5. However, the diagonal matrix terms of order $\epsilon^{2}$ that couple an energy eigenstate with itself $\left(\Delta E^{(n, n)}=0\right)$ have a significant contribution to the energy subband dispersion as in the SLG case which cannot be neglected.

Furthermore, notice how the orders of $\epsilon$ are very similar to the SLG case [in Sec. (3.2)] where off-diagonal terms possess only odd orders and diagonal terms have only even orders of $\epsilon$. This is one of the indications that we can mostly follow the same reasoning as for SLG and hence trust the $(n,-n)$ subband dispersions.

A comparison of the effects of inter-subband coupling terms and diagonal subband contributions on the lowest-subbands $(n= \pm 1)$ can be seen in Fig. (4.6) with parameter values that correspond to a realistic HgTe quantum well ring. We plot the lowest-subbands with and without inter-subband coupling from $n= \pm 2$ and find that the difference between the dispersions is negligible for the region of realistic azimuthal motion $(l \lesssim 50)$. We also find, as expected, that neglecting the diagonal $\epsilon^{2}$ coupling terms yields a less accurate approximation of the energy subband dispersion and thus must be included in the matrix elements of $\mathrm{H}_{\mathrm{SQW}, 1}^{(\tau)}$. However the off-diagonal $\epsilon^{3}$ and higher order $(n,-n)$ contributions to dispersion are seen to be negligible. This all supports our previous reasoning.

Thus, we have shown that the $\mathrm{H}_{\mathrm{SQW}, 1}^{(\tau)}$ can be separated into the block diagonal $(n,-n)$ subband Hamiltonians $\mathrm{H}_{\mathrm{SQW}, 1}^{(\tau, \pm n)}$. The exact form of the subband dispersions is derived in the following section.

\footnotetext{
${ }^{5}$ Remember that they are separated by another subband due to the alternating types of states.
} 


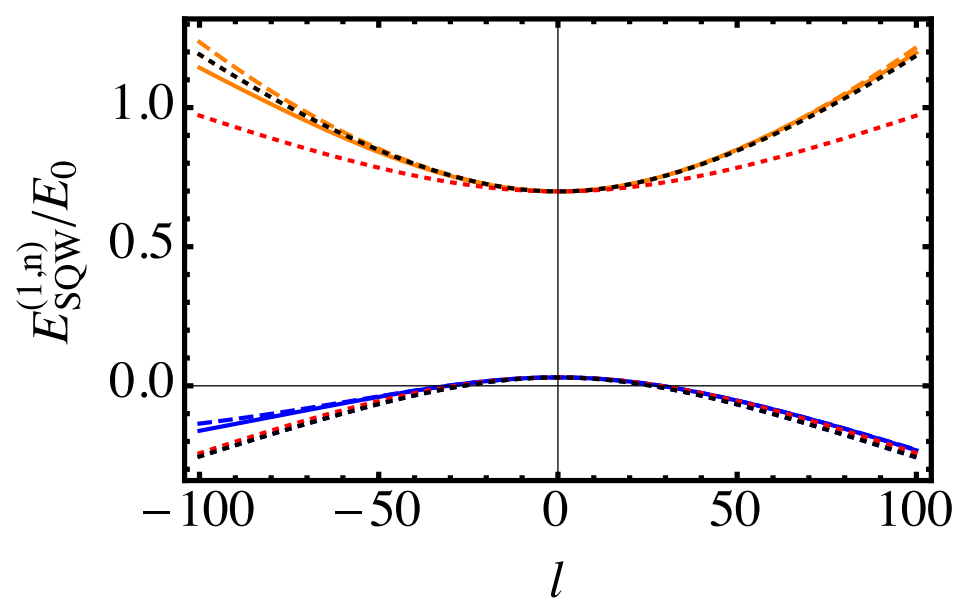

Figure 4.6: Lowest-subband dispersion for a HgTe quantum well with and without coupling from $n= \pm 2$ states. All diagonal and off-diagonal coupling terms between subbands $n= \pm 1, \pm 2$ (solid), $\pm 1,2$ (dashed) and \pm 1 (blackdotted) are included to produce the lowest-subband dispersion. The red-dotted line ignores the diagonal $\epsilon^{2} \mathrm{H}_{\mathrm{SQW}, 1}^{(\tau)}$ terms. Other parameter were set constant at $q_{0} W=5.31, \tilde{D}=-0.746, \xi_{\mathrm{M}}=-0.207$ and $W / R=0.025$.

\subsubsection{Subband energy dispersion}

The block-diagonal $(n,-n)$ subband Hamiltonian up to the first order of $\epsilon=\tilde{W} / \tilde{R}$ in the $(n,-n)$ coupling terms (off-diagonal terms) and $\epsilon^{2}$ in the diagonal terms can be shown to be

$$
\begin{aligned}
& \mathrm{H}_{\mathrm{SQW}}^{(\tau, n)}=E_{0} \times \\
& \left(\begin{array}{cc}
\tilde{E}_{\mathrm{SQW}, 0}^{(\tau, n)}-l \tau\left(\frac{\tilde{W}}{\tilde{R}}\right)^{2} \Lambda_{\mathrm{SQW}}+l^{2}\left(\frac{\tilde{W}}{\tilde{R}}\right)^{2} \Upsilon_{\mathrm{SQW}} & l \tau \frac{\tilde{W}}{\tilde{R}} \Delta_{\mathrm{SQW}} \\
l \tau \frac{\tilde{W}}{\tilde{R}} \Delta_{\mathrm{SQW}}^{*} & \tilde{E}_{\mathrm{SQW}, 0}^{(\tau,-n)}-l \tau\left(\frac{\tilde{W}}{\tilde{R}}\right)^{2} \Lambda_{\mathrm{SQW}}^{\prime}+l^{2}\left(\frac{\tilde{W}}{\tilde{R}}\right)^{2} \Upsilon_{\mathrm{SQW}}^{\prime}
\end{array}\right),
\end{aligned}
$$

where we construct the basis such that $\tilde{E}_{\mathrm{SQW}, 0}^{(\tau, n)}$ is the energy of a type (a) state [Eqn. 4.15a)] and $\tilde{E}_{\mathrm{SQW}, 0}^{(\tau,-n)}$ is the energy of type (b) state [Eqn. 4.15b] 6 .

\footnotetext{
${ }^{6}$ Recall the $\pm n$ subbands are always of different types.
} 
The different components are determined by the following functions

$$
\begin{aligned}
& \Lambda(\delta, \rho, \theta)=\frac{\tilde{c}^{2}}{\tilde{W}^{2}} \sum_{\alpha=1}^{2} \sum_{\beta=1}^{2} \frac{-\tau_{\alpha} \tau_{\beta}}{\cos \left(\frac{\lambda_{\alpha} \tilde{W}}{2}-\theta\right) \cos \left(\frac{\lambda_{\beta} \tilde{W}}{2}-\theta\right)} \times \\
&\left((\delta-\rho)\left[\frac{\tilde{W}}{2} \frac{\cos \left(\frac{\left(\lambda_{\alpha}-\lambda_{\beta}\right) \tilde{W}}{2}\right)}{\lambda_{\alpha}-\lambda_{\beta}}-\frac{\sin \left(\frac{\left(\lambda_{\alpha}-\lambda_{\beta}\right) \tilde{W}}{2}\right)}{\left(\lambda_{\alpha}-\lambda_{\beta}\right)^{2}}\right]\right. \\
&\left.+(\delta+\rho)\left[\frac{\tilde{W}}{2} \frac{\cos \left(\frac{\left(\lambda_{\alpha}+\lambda_{\beta}\right) \tilde{W}}{2}\right)}{\lambda_{\alpha}+\lambda_{\beta}}-\frac{\sin \left(\frac{\left(\lambda_{\alpha}+\lambda_{\beta}\right) \tilde{W}}{2}\right)}{\left(\lambda_{\alpha}+\lambda_{\beta}\right)^{2}}\right]\right),
\end{aligned}
$$

and

$$
\begin{aligned}
\Upsilon(\delta, \rho, \theta, \sigma)=\frac{\tilde{c}^{2}}{\tilde{W}^{2}} \sum_{\alpha=1}^{2} & \sum_{\beta=1}^{2} \frac{\tau_{\alpha} \tau_{\beta}}{\cos \left(\frac{\lambda_{\alpha} \tilde{W}}{2}-\theta\right) \cos \left(\frac{\lambda_{\beta} \tilde{W}}{2}-\theta\right)} \times \\
& \left([1-\tilde{D}-(1+\tilde{D}) \delta \rho] \frac{\sin \left(\frac{\left(\lambda_{\alpha}-\lambda_{\beta}\right) \tilde{W}}{2}\right)}{\lambda_{\alpha}-\lambda_{\beta}}\right. \\
+ & \left.\sigma[1-\tilde{D}+(1+\tilde{D}) \delta \rho] \frac{\sin \left(\frac{\left(\lambda_{\alpha}+\lambda_{\beta}\right) \tilde{W}}{2}\right)}{\lambda_{\alpha}+\lambda_{\beta}}\right),
\end{aligned}
$$

where $\Lambda_{\mathrm{SQW}}=\Lambda\left(\frac{\gamma_{\alpha}}{\sqrt{-\tau_{\alpha}}}, \frac{\sqrt{\gamma_{\beta}}}{-\tau_{\beta}}, 0\right), \Lambda_{\mathrm{SQW}}^{\prime}=\Lambda\left(\tau_{\beta} \gamma_{\beta} \sqrt{ } \overline{-\tau_{\beta}}, \tau_{\alpha} \gamma_{\alpha} \sqrt{-\tau_{\alpha}}, \frac{\pi}{2}\right), \Upsilon_{\mathrm{SQW}}=$ $\Upsilon\left(\frac{\gamma_{\alpha}}{\sqrt{-\tau_{\alpha}}}, \frac{\sqrt{\gamma_{\beta}}}{-\tau_{\beta}}, 0,1\right)$ and $\Upsilon_{\mathrm{SQW}}^{\prime}=\Upsilon\left(\tau_{\beta} \gamma_{\beta}{ }^{\sqrt{-\tau_{\beta}}}, \tau_{\alpha} \gamma_{\alpha} \sqrt{-\tau_{\alpha}}, \frac{\pi}{2},-1\right)$. The offdiagonal terms are given by

$$
\begin{aligned}
& \Delta_{\mathrm{SQW}}=\frac{c_{1} c_{2}^{\prime}}{\tilde{W}} \sum_{\alpha=1}^{2} \sum_{\beta=1}^{2} \frac{\tau_{\alpha} \tau_{\beta}^{\prime}}{\cos \left(\frac{\lambda_{\alpha} \tilde{W}}{2}\right) \sin \left(\frac{\lambda_{\beta}^{\prime} \tilde{W}}{2}\right)} \times \\
& {\left[\frac{\frac{\gamma_{\alpha}}{\sqrt{-\tau_{\alpha}}}+\tau_{\beta}^{\prime} \gamma_{\beta}^{\prime} \sqrt{-\tau_{\beta}^{\prime}}}{\lambda_{\alpha}-\lambda_{\beta}^{\prime}} \sin \left(\frac{\left(\lambda_{\alpha}-\lambda_{\beta}^{\prime}\right) \tilde{W}}{2}\right)-\frac{\frac{\gamma_{\alpha}}{\sqrt{-\tau_{\alpha}}}-\tau_{\beta}^{\prime} \gamma_{\beta}^{\prime} \sqrt{-\tau_{\beta}^{\prime}}}{\lambda_{\alpha}+\lambda_{\beta}^{\prime}} \sin \left(\frac{\left(\lambda_{\alpha}+\lambda_{\beta}^{\prime}\right) \tilde{W}}{2}\right)\right] .}
\end{aligned}
$$


The eigenenergies, $\tilde{E}_{\mathrm{SQW}}^{(\tau, \pm n)}$, of the $\pm n$ subbands are found to be given by

$$
\begin{aligned}
& \tilde{E}_{\mathrm{SQW}}^{(\tau, \pm n)}= \\
& \frac{\tilde{E}_{\mathrm{SQW}, 0}^{(\tau, n)}+\tilde{E}_{\mathrm{SQW}, 0}^{(\tau,-n)}-l \tau\left(\frac{\tilde{W}}{\tilde{R}}\right)^{2}\left(\Lambda_{\mathrm{SQW}}+\Lambda_{\mathrm{SQW}}^{\prime}\right)+l^{2}\left(\frac{\tilde{W}}{\tilde{R}}\right)^{2}\left(\Upsilon_{\mathrm{SQW}}+\Upsilon_{\mathrm{SQW}}^{\prime}\right)}{2} \\
& \pm\left[\left(\frac{\tilde{E}_{\mathrm{SQW}, 0}^{(\tau, n)}-\tilde{E}_{\mathrm{SQW}, 0}^{(\tau,-n)}-l \tau\left(\frac{\tilde{W}}{\tilde{R}}\right)^{2}\left(\Lambda_{\mathrm{SQW}}-\Lambda_{\mathrm{SQW}}^{\prime}\right)+l^{2}\left(\frac{\tilde{W}}{\tilde{R}}\right)^{2}\left(\Upsilon_{\mathrm{SQW}}-\Upsilon_{\mathrm{SQW}}^{\prime}\right)}{2}\right)^{2}\right. \\
& \left.+l^{2}\left(\frac{\tilde{W}}{\tilde{R}}\right)^{2}\left|\Delta \Delta_{\mathrm{SQW}}\right|^{2}\right]^{\frac{1}{2}} .
\end{aligned}
$$

Unfortunately, so far we have discovered no simplified expressions for the components of the subband energy dispersion, unlike the dispersion equations for SLG and TMD. This is due to the extra layer of complication that is present due to the existence of wing states, as seen in Eqns. 4.35 and 4.38, and the lack of energy-reflection symmetry for a non-zero $\tilde{D}$.

Now that the general subband dispersions have been obtained, we proceed to study the qualitative features of these dispersions. To do this, we consider the simplified $\tilde{D}=0$ case first. Once we have understood this instance, we proceed to the more general $\tilde{D} \in 0$ situation.

\section{Energy-reflections symmetric case}

Firstly, we must note that for $\tilde{D}=0$, the subband dispersions can be significantly simplified since $\tilde{E}_{\mathrm{SQW}, 0}^{(\tau, n)}=-\tilde{E}_{\mathrm{SQW}, 0}^{(\tau,-n)}, \Lambda_{\mathrm{SQW}}=\Lambda_{\mathrm{SQW}}^{\prime}$ and $\Upsilon_{\mathrm{SQW}}=$ $-\Upsilon_{\mathrm{SQW}}^{\prime}$. Eqn. 4.48 can thus be expressed as

$$
\begin{aligned}
\tilde{E}_{\mathrm{SQW}}^{(\tau, \pm n)}= & -l \tau\left(\frac{\tilde{W}}{\tilde{R}}\right)^{2} \Lambda_{\mathrm{SQW}} \\
& \pm \sqrt{\left[\tilde{E}_{\mathrm{SQW}, 0}^{(\tau, n)}+l^{2}\left(\frac{\tilde{W}}{\tilde{R}}\right)^{2} \Upsilon_{\mathrm{SQW}}\right]^{2}+l^{2}\left(\frac{\tilde{W}}{\tilde{R}}\right)^{2}\left|\Delta_{\mathrm{SQW}}\right|^{2}}
\end{aligned}
$$


In Fig. 4.7), for $\tilde{D}=0$, we depict the relative positions of the $\tau$ flavour subband energy dispersions for $\xi_{\mathrm{M}} q_{0} W=-2$ (inverted gap with TPESs) and $\xi_{\mathrm{M}} q_{0} W=0$ (non-inverted gap), as a direct comparison to Fig. (3.3) for the SLG case. Although visibly different from Fig. (3.3), once again, we discover that for $n=2$ the $\tau$ flavour dispersions swap positions, i.e. $\tau=1$ dispersion shifts from being on the left (in inverted gap $\xi_{\mathrm{M}} q_{0} W=$ -2 ) to being on the right (non-inverted gap $\xi_{\mathrm{M}} q_{0} W=0$ ) of the $\tau=-1$ dispersion as they transition between the two topological phases. However, this swapping phenomenon does not occur for the $n=1$ subband which indicates a qualitative difference between the topological and trivial states.

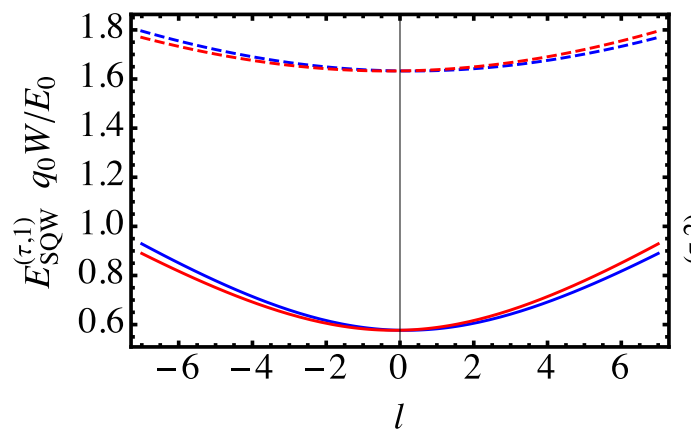

(a) lowest-subband

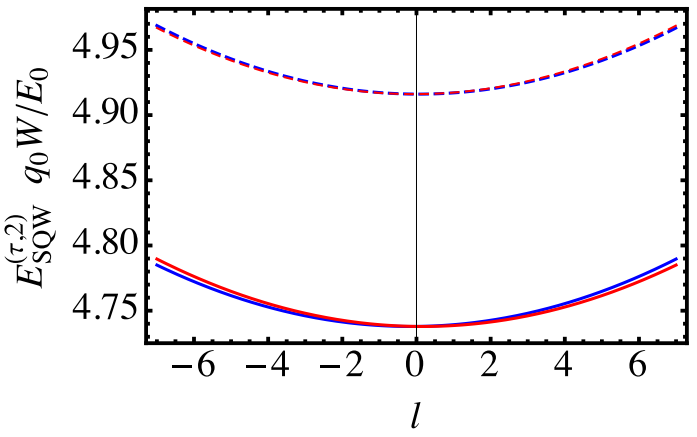

(b) Second lowest-subband

Figure 4.7: Plot of general subband energy dispersion of lowest (a) and second lowest (b) subbands for $q_{0} W=53.1, W / R=0.1, \tilde{D}=0, \xi_{\mathrm{M}} q_{0} W=$ -2 (solid) and 0 (dashed) with both flavours $\tau=1$ (blue) and -1 (red) at $\boldsymbol{\Gamma}$. Notice that for $(\mathbf{b})$ we still have a gap because $E_{\mathrm{SQW}}^{(\tau, 1)} \boldsymbol{\sigma} 0$ due to the confinement-effects that increase the confinement energies in the material.

We expect, as in the SLG case, that there is a value of $\xi_{\mathrm{M}} q_{0} W$ between $\xi_{\mathrm{M}} q_{0} W=-2$ and 0 at which the two flavours have an identical energy dispersion, i.e. $E_{\mathrm{SQW}}^{(\tau, n>1)}=E_{\mathrm{SQW}}^{(-\tau, n>1)}$, thus leading to a zero shift between the dispersions. The value at which this occurs corresponds to when $\Lambda_{\mathrm{SQW}}=$ $\Lambda_{\mathrm{SQW}}^{\prime}=0$ which causes zero $\tau$ dependence in Eqn. 4.49p (hence no shift between $\pm \tau$ flavour dispersions). Additionally, by observing the subband dispersion equation [Eqn. (4.49)] one can determine that the shift in $l$ values between the different $\pm \tau$ flavour subband dispersions is directly proportional to the size of $\Lambda_{\mathrm{SQW}}$. This will be explicitly shown in Chapter 6 .

We plot $\Lambda_{\mathrm{SQW}}$ as a function of the gap size in Fig. 4.8). This allows us to gain an insight into the magnitudes of the relative shift between the $\tau$ 
dispersions. Transitions from negative to positive values of $\Lambda_{\mathrm{SQW}}$ only arise for subbands with $n>1$, similarly to SLG. Thus we can see points at which $\Lambda_{\mathrm{SQW}}=0$ for these subbands. Additionally, the lowest-subband never swaps positions of the two flavour dispersion curves as we transition between the two regimes. This striking feature seems to be a signature of the topological nature of the state. Additionally, $\Lambda_{\mathrm{SQW}}$ as a function of $\xi_{\mathrm{M}}$ monotonically decreases for $n=1$ while it monotonically increases for $n>1$.

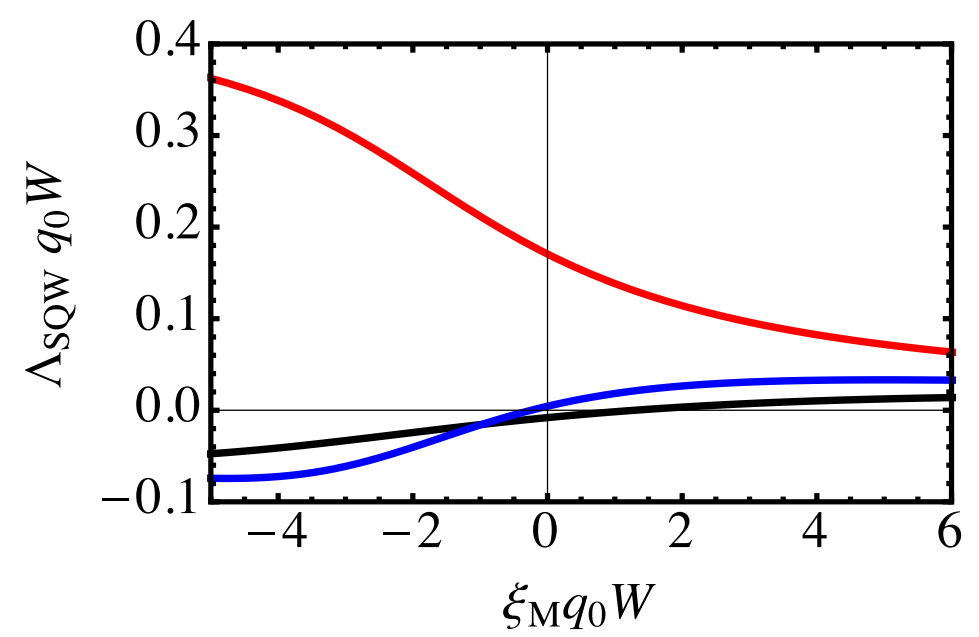

Figure 4.8: $\Lambda_{\mathrm{SQW}}$ as function of $\xi_{\mathrm{M}}$ at a fixed width $q_{0} W=26.6$ for the three lowest-subbands: $n=1$ (red), 2 (blue) and 3 (black). Other parameters were held constant at $W / R=0.1$ and $\tilde{D}=0$. We choose to plot $\Lambda_{\mathrm{SQW}} q_{0} W$ since it is then unit-wise equivalent to $\Gamma_{\mathrm{SLG}}$ from Fig. (3.4).

Now, we shall explore the gap value at which $\Lambda_{\mathrm{SQW}}=0$. Recall from Sec. (3.3.1) that, in the SLG case, the zero point corresponded perfectly with the transition point between the topological and trivial phases of the material. However, contrary to the SLG case, the "swapping" point of the $\tau$ flavours in SQW does not occur at $\xi_{\mathrm{Mc}} q_{0} W \approx-1$ [Eqn. (4.28)] and hence does not correspond to the transition point from the trivial to the topological regime as discussed in Sec. 4.1.1.).

We have now seen some of the different features between the lowest-subband and the higher subbands for an energy-reflection symmetric SQW QR. Now, we move on to analysing the differences that arise due to a non-zero $\tilde{D}$. 


\section{Non-energy-reflection symmetric case}

For $\tilde{D} \sigma 0$, we once again show the swapping of $\tau$ flavour dispersions for the $n=2$ subband in Fig. 4.9 as we tune from a deeply inverted gap $\left[\xi_{\mathrm{M}} q_{0} W=-7\right.$ in Fig. 4.9a)] to a zero gap $\left[\xi_{\mathrm{M}} q_{0} W=0\right.$ in Fig. 4.9b)].

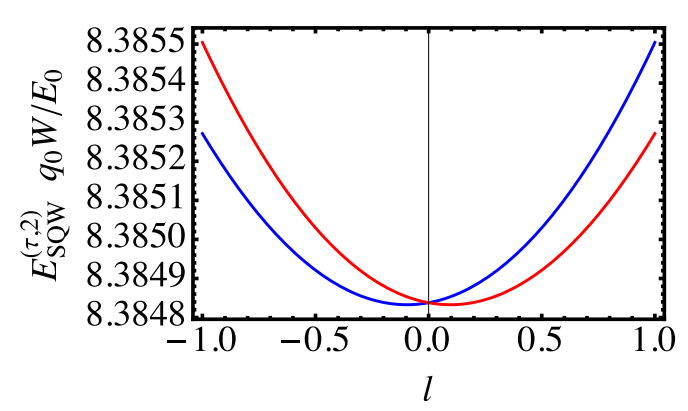

(a) $\xi_{\mathrm{M}} q_{0} W=-7$

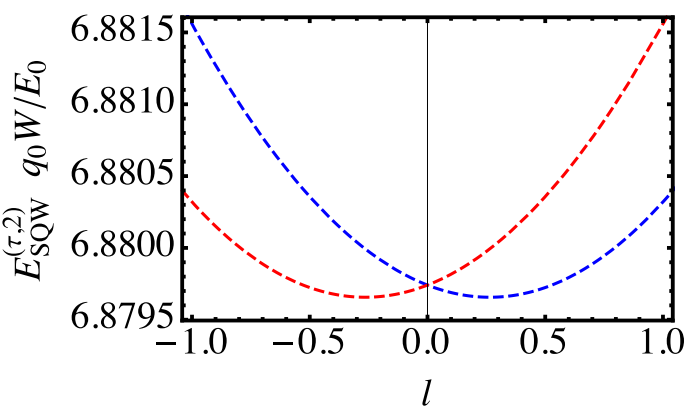

(b) $\xi_{\mathrm{M}} q_{0} W=0$

Figure 4.9: Plot of a $S Q W Q R n=2$ subband energy dispersion when (a) $\xi_{\mathrm{M}} q_{0} W=-7$ and (b) $\xi_{\mathrm{M}} q_{0} W=0$ with both flavours $\tau=1$ (blue) and -1 (red). Other parameters were held constant at $q_{0} W=26.6, W / R=0.1$ and $\tilde{D}=-0.746$.

We also depict the $n=1$ subband dispersions in Fig. 4.10 for $\xi_{\mathrm{M}} q_{0} W=-7$ and $\xi_{\mathrm{M}} q_{0} W=0$. Here we see that the subband flavours do not swap for $n=1$ which shows the difference between the topological and trivial subbands. Additionally for $\xi_{\mathrm{M}} q_{0} W=-7$, we also plot the $n=-1$ subband so that one can see the gapless nature of subbands in the deeply inverted gap regime. One can also observe that due to the lack of energy-reflection symmetry, we see a shift in the energies are not symmetrically distributed around $\tilde{E}_{\mathrm{SQW}}^{(\tau, n)}=0$, as previously discussed in Sec. 4.1.1). In Fig. 4.10 we also observe that when the band-gap $\xi_{\mathrm{M}}$ is zero, there is still a gap between the $n= \pm 1$ subbands due to the confinement effects, as in the $\tilde{D}=0$ case.

Contrary to the energy-reflection symmetric cases, the $\tau$ flavour dispersion shift now differs not only for different $n>0$ subbands but also for $\pm n$ subbands as we will see in Fig. 4.11. In this case, for energies $E_{\mathrm{SQW}}^{(\tau, n)} \approx$ $E_{\mathrm{SQW}, 0}^{(\tau, n)} 7$ the shift between the two flavours is approximately proportional to $\Lambda_{\mathrm{SQW}}$ for type (a) and $\Lambda_{\mathrm{SQW}}^{\prime}$ for type (b) subbands ${ }^{8}$. In particular, the

\footnotetext{
${ }^{7}$ This is generally true for the regime of interest since a small change in the energy causes a large change in the azimuthal quantum number [see as an example Fig. 4.77]. Since we deal with $|l| \lesssim 50$, we stay in this regime.

${ }^{8}$ This will be definitively shown in Chapter 6
} 


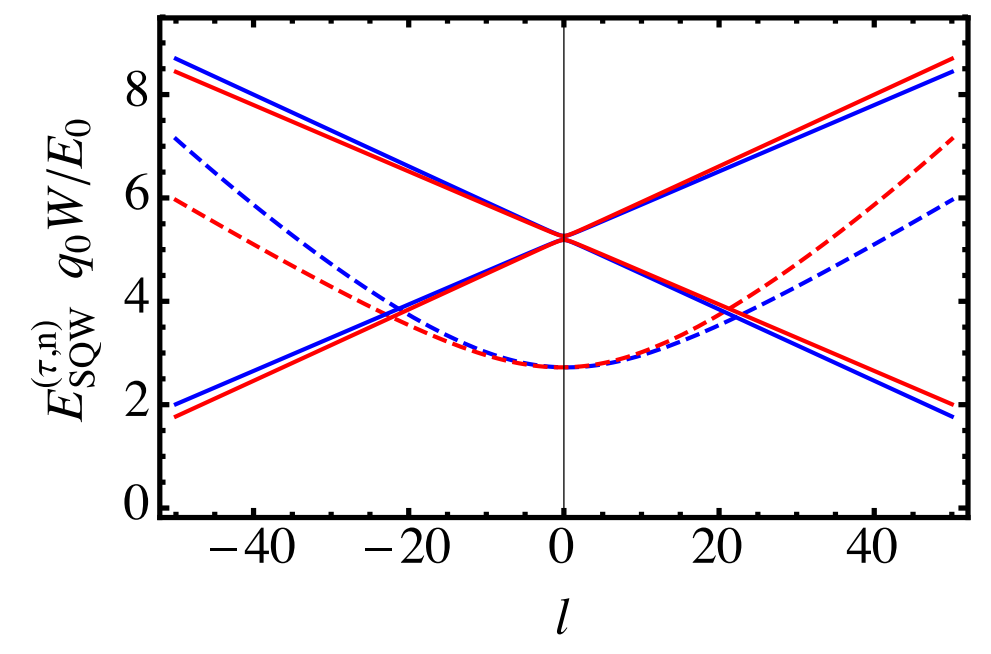

Figure 4.10: Plot of a $S Q W Q R n= \pm 1$ subband energy dispersions for $\xi_{\mathrm{M}} q_{0} W=-7$ (solid) and $\xi_{\mathrm{M}} q_{0} W=0$ (dashed) with both flavours $\tau=1$ (blue) and -1 (red). Other parameters were held constant at $q_{0} W=26.6$, $W / R=0.1$ and $\tilde{D}=-0.746$. The $n=-1$ subband of $\xi_{\mathrm{M}} q_{0} W=0$ is not included since it has negative energies and would unnecessarily complicate the figure.

zero shift difference between the two flavours occurs when either $\Lambda_{\mathrm{SQW}}=0$ or $\Lambda_{\mathrm{SQW}}^{\prime}=0$ for their respective subband types.

We plot $\Lambda_{\mathrm{SQW}}$ [for type (a) subbands] and $\Lambda_{\mathrm{SQW}}^{\prime}$ [for type (b) subbands] as a function of the gap size for $n= \pm 1, \pm 2, \pm 3$ in Fig. (4.11. We see that the $|n|>1$ subbands transition from negative to positive values of $\Lambda_{\mathrm{SQW}}$ (or $\Lambda_{\mathrm{SQW}}^{\prime}$ ) as the gap size is tuned from a deeply inverted gap to a non-inverted gap. However, as in the $\tilde{D}=0$ case, it can be seen that the $n= \pm 1$ subbands are always positive and do not possess a $\Lambda_{\mathrm{SQW}}=0$ or $\Lambda_{\mathrm{SQW}}^{\prime}=0$ point, respectively. Furthermore, the $\Lambda_{\mathrm{SQW}}$ and $\Lambda_{\mathrm{SQW}}^{\prime}$ values of lowest-subbands monotonically decrease while the higher subbands seem to generally increase 9 . Since these features are present in all cases considered thus far, it further demonstrates the curious and unique nature of the topological subbands as compared to the trivial ones.

As shown in Fig. 4.11), we find that the swapping points (i.e. $\Lambda_{\mathrm{SQW}}=0$ or $\left.\Lambda_{\mathrm{SQW}}^{\prime}=0\right)$ do not occur at $\xi_{\mathrm{Mc} \pm} q_{0} W$, as given by Eqn. (4.28) for large $q_{0} W$, and as such is not a clear indicator that separates the topological from

\footnotetext{
${ }^{9}$ Unlike the $\tilde{D}=0$ case, the higher subbands do not monotonically increase anymore as we can see a bump in the $n=-2$ subbands.
} 


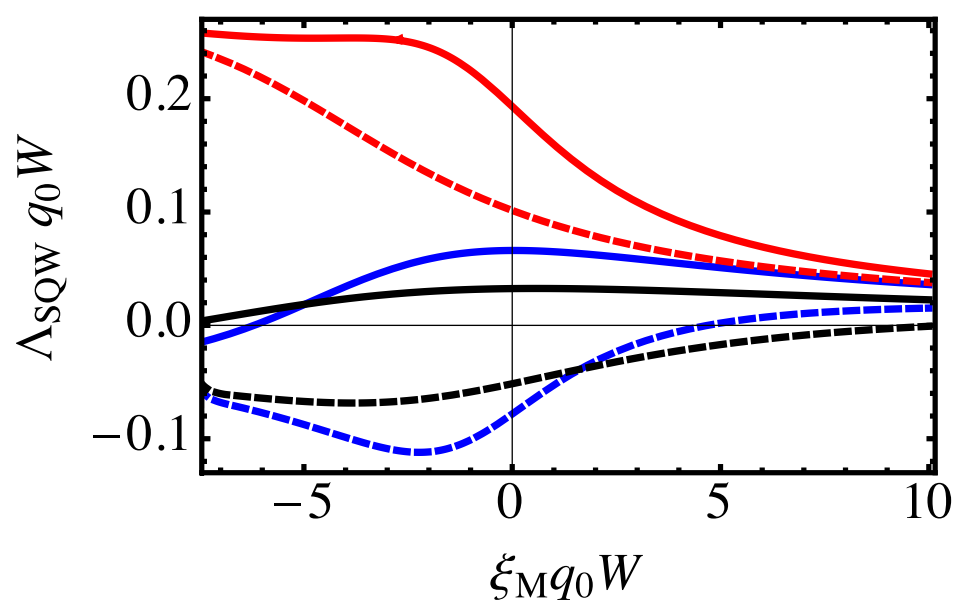

Figure 4.11: $\Lambda_{\mathrm{SQW}}$ for type (a) states and $\Lambda_{\mathrm{SQW}}^{\prime}$ for type (b) states as a function of gap size $\xi_{\mathrm{M}} q_{0} W$ for a $S Q W Q R$ where the different subbands are given by $n= \pm 1$ (solid/dashed red), \pm 2 (solid/dashed blue) and \pm 3 (solid/dashed black). Parameter values were kept constant at $q_{0} W=26.6$, $\tilde{D}=-0.746$ and $W / R=0.1$.

the trivial phase as was the case for SLG. We also notice that the swapping points are notably shifted compared to both the SLG and SQW $\tilde{D}=0$ case.

We will continue our discussion with respect to the differences between the topological and trivial regimes as we explore these concepts in further detail when investigating the QR Berry phase in Chapter 6. We have now comprehensively explored the radial properties of the SQW ring system such as the change in its topological nature when confined. However, a complete insight into the physical consequences due to the azimuthal motion determined by $H_{\alpha, 1}^{(\tau)}$ is still missing. Thus we find it a necessity to explore the transport properties of SQW (and SLG, TMD) ring systems in order to truly see the effects of the $H_{\alpha, 1}^{(\tau)}$ Hamiltonian. In the next chapter we determine the form of the conductance for these QR systems. 


\section{Chapter 5}

\section{Conductance}

This chapter focuses on the conductance properties of the ring-confined systems and is divided into two parts.

In the first part of this chapter, we concentrate on developing the general formalism and properties of the conductance without specifically applying this formulism to the individual materials. We begin by deriving the nonmaterial specific forms of parameters: $\theta \leftrightarrow \theta_{\mathrm{g}}^{(\tau, n)} / 2$ and $\phi_{\mathrm{s}} \leftrightarrow \chi_{\mathrm{s}}^{(\tau, n)}$, as was discussed in Chapter 2. These parameters are then expressed in a simplified form, via symmetry arguments regarding real and pseudo-spin degrees of freedom, and implemented into the conductance. After this, we explore and suggest experimental strategies for determining these dynamic and geometric phases at zero and non-zero magnetic fields.

In the second part, we determine the explicit forms of $\theta_{\mathrm{g}}^{(\tau, n)}$ and $\chi_{\mathrm{s}}^{(\tau, n)}$ for the massless-Dirac SLG, TMD and SQW rings. The dependence of the conductance as a function of $\phi$ and $E_{\text {in }}$ for these materials is presented and the appearance of resonance peaks and troughs are discussed. Finally, we explore the behaviour of the $\tau$ flavour conductance peaks and their relative positions and connect this back to the $\tau$ flavour energy dispersion swapping that was discovered in the Chapters 3 and 4. 


\subsection{General conductance derivation}

By construction, in the QRs, we have an anticlockwise and clockwise moving partial wave that are classified by the angular quantum numbers $l_{+}^{(\tau, n)}$ and $l_{-}^{(\tau, n)}$, respectively, as can be seen in Fig. 5.1. When the ring is connected to leads, an electron injected into junction $j$ from a lead state $\mid \nu_{j}$ i with injection energy $E_{\text {in }}>0$ will be in the interferometer state

$$
\begin{aligned}
\mid E_{\text {in }}, \nu_{j} \mathbf{i} & =c_{+}^{\left(\nu_{j}\right)} \mathrm{U}_{\tau}\left(\varphi-\varphi_{j}\right) \mathrm{e}^{i l_{+}^{(\tau, n)}\left(\varphi-\varphi_{j}\right)} \mid \Phi_{l_{+}^{(\tau, n)}, \alpha}^{(n)} \mathbf{i} \\
& +c_{-}^{\left(\nu_{j}\right)} \mathrm{U}_{\tau}\left(\varphi-\left[\varphi_{j}+2 \pi\right]\right) \mathrm{e}^{i l_{-}^{(\tau, n)}\left(\varphi-\left[\varphi_{j}+2 \pi\right]\right)} \mid \Phi_{l_{-}^{(\tau, n)}, \alpha}^{(n)} \mathbf{i}
\end{aligned}
$$

where we adopt the convention that $-\pi \leq \varphi_{j}<\pi$ and $\varphi>\varphi_{j}$ and $c_{ \pm}^{\left(\nu_{j}\right)}$ correspond to the scattering amplitudes $\left\{\beta_{j}, \beta_{j}^{\prime}\right\}$ and $\left\{\gamma_{j}, \gamma_{j}^{\prime}\right\}$. At this point we remind the reader that we have defined $\bigcup_{\tau}(\varphi) \equiv \exp \left\{-i \tau \frac{\sigma_{3}}{2} \varphi\right\}$ as in Chapter 2. Additionally, note that the phase shift of $2 \pi$ between the rightmoving and left-moving partial waves (having azimuthal quantum numbers $l_{+}^{(\tau, n)}$ and $l_{-}^{(\tau, n)}$, respectively) is required to account for the initial conditions associated with electron injection.

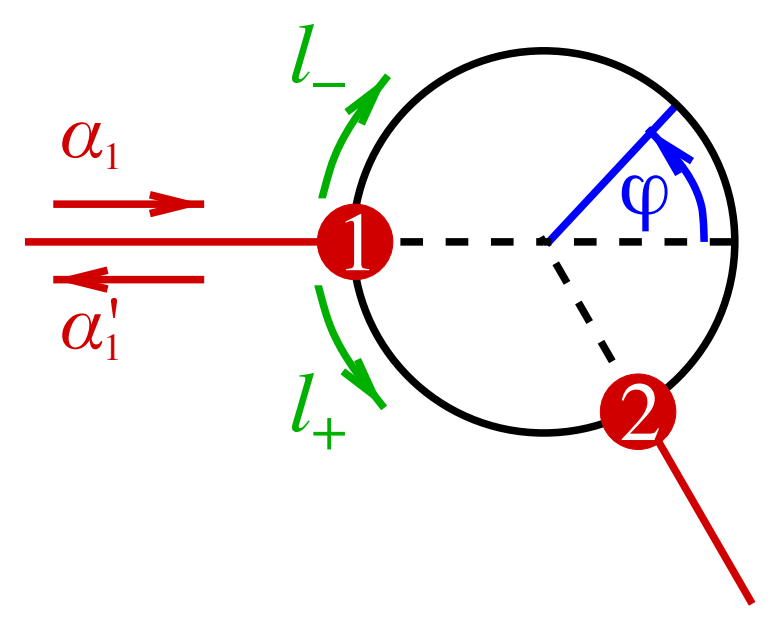

Figure 5.1: An injected wave with amplitude $\alpha_{1}$ is partially reflected via a wave with amplitude $\alpha_{1}^{\prime}$ and partially transmitted into the ring via waves that are categorised by their angular quantum number $l_{+}^{(\tau, n)}$ and $l_{-}^{(\tau, n)} \cdot l_{+}^{(\tau, n)}$ and $l_{-}^{(\tau, n)}$ are constructed to travel anticlockwise and clockwise, respectively.

Given an electron injection energy $E_{\text {in }}$, the $l_{ \pm}^{(\tau, n)}$ are determined from the re- 
lation $E_{\mathrm{in}}=E_{\alpha}^{(n)}\left(l_{ \pm}^{(\tau, n)}\right)$. The anticlockwise or clockwise movement associated with $l_{ \pm}^{(\tau, n)}$ is determined via the velocity $v_{l} \propto \operatorname{sgn}\left(\partial E_{\alpha}^{(n)} / \partial l\right)= \pm 1$, respectively. Note that, for the SLG, TMD and SQW ring conductors considered here, we generally have $l_{+}^{(\tau, n)} 6-l_{-}^{(\tau, n)}$ even with magnetic flux $\phi=0$ [see Figs. (3.2) and (4.10]). We adopt the same notation as in Ref. 35] by using the states $\mathrm{U}_{\alpha}\left(-\varphi_{j}\right) \mid \Phi_{l_{\sigma}^{(\tau, n)}, \alpha}^{(n)} \mathbf{i}$ as a basis and observe that $\mathrm{U}_{\alpha}(2 \pi)=e^{i \zeta_{\alpha} \pi}$ with $\zeta_{\alpha}=1$ for the materials that are of interest to us 1 . Then, using Eqn. (5.1) in the new basis, we locally match the lead and ring states at the junctions and determine the transfer matrices through the upper and lower ring parts connecting the junctions 1 and 2. These are found to be

$$
\begin{aligned}
& \left(\begin{array}{l}
\beta_{2} \\
\beta_{2}^{\prime}
\end{array}\right)=\mathrm{e}^{-i \theta_{1}^{(\tau, n)}}\left(\begin{array}{cc}
\mathrm{e}^{i \chi_{1}^{(\tau, n)}} & 0 \\
0 & \mathrm{e}^{-i \chi_{1}^{(\tau, n)}}
\end{array}\right)\left(\begin{array}{c}
\beta_{1}^{\prime} \\
\beta_{1}
\end{array}\right), \\
& \left(\begin{array}{l}
\gamma_{1} \\
\gamma_{1}^{\prime}
\end{array}\right)=\mathrm{e}^{-i \theta_{2}^{(\tau, n)}}\left(\begin{array}{cc}
\mathrm{e}^{i \chi_{2}^{(\tau, n)}} & 0 \\
0 & \mathrm{e}^{-i \chi_{2}^{(\tau, n)}}
\end{array}\right)\left(\begin{array}{l}
\gamma_{2}^{\prime} \\
\gamma_{2}
\end{array}\right),
\end{aligned}
$$

with the phases

$$
\begin{aligned}
\theta_{1}^{(\tau, n)} & =\frac{l_{+}^{(\tau, n)}+l_{-}^{(\tau, n)}}{2}\left(\varphi_{1}-\varphi_{2}\right)+\pi\left(l_{+}^{(\tau, n)}+l_{-}^{(\tau, n)}+1\right) \\
\theta_{2}^{(\tau, n)} & =\frac{l_{+}^{(\tau, n)}+l_{-}^{(\tau, n)}}{2}\left(\varphi_{2}-\varphi_{1}\right) \\
\chi_{1}^{(\tau, n)} & =\frac{l_{+}^{(\tau, n)}-l_{-}^{(\tau, n)}}{2}\left(2 \pi+\varphi_{1}-\varphi_{2}\right), \\
\chi_{2}^{(\tau, n)} & =\frac{l_{+}^{(\tau, n)}-l_{-}^{(\tau, n)}}{2}\left(\varphi_{2}-\varphi_{1}\right) .
\end{aligned}
$$

where $\alpha_{i}, \beta_{i}$ and $\gamma_{i}$ are denoted in Fig. 2.1) of Chapter 2. The transmission function (and therefore the electric conductance) through the ring is found to be a function of the geometric phase

$$
\begin{aligned}
\theta_{\mathrm{g}}^{(\tau, n)} & =\theta_{1}^{(\tau, n)}+\theta_{2}^{(\tau, n)} \\
& =\pi\left[l_{+}^{(\tau, n)}(\phi)+l_{-}^{(\tau, n)}(\phi)+1\right], \\
& \equiv \pi\left[l_{+}^{(\tau, n)}(0)+l_{-}^{(\tau, n)}(0)+1\right]+2 \pi \frac{\phi}{\phi_{0}}
\end{aligned}
$$

\footnotetext{
${ }^{1}$ As an example of a material with a different factor, bi-layer graphene has $\zeta_{\alpha}=0$.
} 
that depends explicitly on the magnetic flux $\phi$ penetrating the ring, and the flux-independent dynamic phases $\chi_{1,2}^{(\tau, n)}$. For a symmetrically connected ring (i.e., when $\varphi_{2}-\varphi_{1}=\pi$ ), we have

$$
\chi_{1}^{(\tau, n)}=\chi_{2}^{(\tau, n)} \equiv \chi_{\mathrm{s}}^{(\tau, n)}=\left(l_{+}^{(\tau, n)}-l_{-}^{(\tau, n)}\right) \frac{\pi}{2} .
$$

The transmission probability of the ring is then given by Eqn. (2.40) (originally derived in Ref. 35$])$ with the correspondences $\phi_{\mathrm{s}} \leftrightarrow \chi_{\mathrm{s}}^{(\tau, n)}$ and $\theta \leftrightarrow$ $\theta_{\mathrm{g}}^{(\tau, n)} / 2$, that is

$$
\begin{aligned}
& T\left(\chi_{\mathrm{s}}^{(\tau, n)}, \theta_{\mathrm{g}}^{(\tau, n)}, \epsilon_{c}\right)= \\
& \frac{4 \epsilon_{c}^{2} \sin ^{2} \chi_{\mathrm{s}}^{(\tau, n)} \cos ^{2}\left(\theta_{\mathrm{g}}^{(\tau, n)} / 2\right)}{\left[a^{2}+b^{2} \cos \theta_{\mathrm{g}}^{(\tau, n)}-\left(1-\epsilon_{c}\right) \cos 2 \chi_{\mathrm{s}}^{(\tau, n)}\right]^{2}+\epsilon_{c}^{2} \sin ^{2} 2 \chi_{\mathrm{s}}^{(\tau, n)}} .
\end{aligned}
$$

Remember from the definitions in Chapter 2 that $\epsilon_{c}$ is a coupling parameter that arises from the $\mathrm{S}$ matrix which couples the source and drain leads to the ring.

The total conductance is obtained by summing over transmission probabilities in all open channels that are associated with the real-spin and $\tau$ flavour degrees of freedom as stated in Eqn. 2.29). In SLG and SQW rings, there is no real-spin dependence in the dynamics considered here and thus is accounted for by a simple degeneracy factor. In TMD rings, due to real spinsplitting, the summation of contributions from real-spin is non-trivial [see Fig. (3.5)]. Since particles with the same $\tau s_{3}$ factor have similar phases and transmissions (as we will see in the following sections), this can simplify our calculations. However, we still have to sum over all $\tau$ and $s_{3}$ separately for TMD QRs.

The ring transmission has a $\tau$ or $\tau s_{3}$ flavour dependence because, by virtue of time-reversal invariance:

$$
l_{ \pm}^{(\eta, n)}(0)=-l_{\mp}^{(-\eta, n)}(0)
$$

where $\eta=\tau$ for SLG \& SQW and $\eta=\left\{\tau, s_{3}= \pm \tau\right\}$ for TMD 2 , Therefore

\footnotetext{
${ }^{2}$ Here for TMD we have just added an extra index to the terms, e.g. $l_{ \pm}^{(\eta, n)} \equiv l_{ \pm}^{\left(\tau, s_{3}, n\right)}$. Additionally, $s_{3}$ can be expressed as $\mathbf{\pm} \tau$ to simplify our future expression.
} 
we find

$$
\begin{aligned}
& \chi_{\mathrm{s}}^{(\eta, n)}=\chi_{\mathrm{s}}^{(-\eta, n)} \\
& \theta_{\mathrm{g}}^{(\eta, n)}=2 \pi\left(\frac{\phi}{\phi_{0}}+\frac{1}{2}\right)+\tau \delta \theta_{\mathrm{g}}^{(n)} .
\end{aligned}
$$

where we define

$$
\delta \theta_{\mathrm{g}}^{(n)}=\pi\left[l_{+}^{(1, n)}(0)+l_{-}^{(1, n)}(0)\right]
$$

for SLG \& SQW and

$$
\delta \theta_{\mathrm{g}}^{(n)}=\pi\left[l_{+}^{(1, \pm 1, n)}(0)+l_{-}^{(1, \pm 1, n)}(0)\right]
$$

for TMD. The quantity $\delta \theta_{\mathrm{g}}^{(n)}$ is what we call the correction to the confinementinduced Berry phase. This is because although $\theta_{\mathrm{g}}^{(\eta, n)}$ nominally includes the Aharanov-Bohm phase, $2 \pi \phi / \phi_{0}$, and spin- $1 / 2$ phase, $\pi$, the extra ring correction term, $\delta \theta_{\mathrm{g}}^{(n)}$, has so far been given very little attention, if any at all. However, this term turns out to play an important role in the form of the conductances which we shall see in the later half of this chapter. Looking closely, we also realise that $\delta \theta_{\mathrm{g}}^{(n)}$ is proportional to the shift between the $\tau$ flavour dispersions that we analysed for SLG, TMD and SQW QRs and must thus also be related to the quantity $\Gamma_{\alpha}$ (or $\Lambda_{\mathrm{SQW}}$ and $\Lambda_{\mathrm{SQW}}^{\prime}$ for SQW QRs) that governs this shift. This property is also explored in the later sections.

The ring conductance for SLG and SQW QRs are thus given by

$$
G=\frac{e^{2}}{\pi \hbar} \sum_{\tau=-1,1} T\left(\chi_{\mathrm{s}}^{(\eta, n)}, \theta_{\mathrm{g}}^{(\eta, n)}, \epsilon\right)
$$

For TMDs, we use the modified version:

$$
G=\frac{e^{2}}{2 \pi \hbar} \sum_{\tau, s_{3}=-1,1} T\left(\chi_{\mathrm{s}}^{\left(\tau, s_{3}, n\right)}, \theta_{\mathrm{g}}^{\left(\tau, s_{3}, n\right)}, \epsilon\right)
$$

that takes into account the real spin solutions separately.

In the next section we present measurement strategies that may be implemented by experimentalists to detect the dynamic and geometric phases. 


\subsubsection{Detection methods}

An important feature of the conductance is the existence of peaks and troughs (i.e. resonances) for certain configurations of non-material related parameters ( $\phi$ and $E_{\text {in }}$ ). If we can determine the connection between these configurations and the dynamic and geometric phases, then we may find a simple method to measure these quantities. In particular, there would be an interest in measuring the correction to the confinement-induced Berry phase, $\delta \theta_{\mathrm{g}}^{(n)}$, for which we will develop specific measurement strategies in the following sections.

For a fixed value of $\phi$, the magnitude of conductance changes when $E_{\text {in }}$ is varied. We find that minima usually occurs when $\chi_{\mathrm{s}}^{(\eta, n)}=p \pi$ where $p \in \mathbb{Z}$ which causes the $\sin \left(\chi_{\mathrm{s}}^{(\eta, n)}\right)=0$ in the transmission function [see Eqn. [5.6)]. The energies at which these zero conductances occur are denoted by $E_{\min }^{(p)}$. There are exceptions to this feature when the denominator is also zero which we explore below.

In the case of a varying $\phi$, we can observe that the conductance has a periodicity of $\phi_{0}$. We have seen in Eqns. (5.11) and (5.12) that the conductance is an accumulation of individual contributions from the $\pm \eta$ flavours, which exhibit different $\theta_{\mathrm{g}}^{(\eta, n)}$ values [see Eqn. $\left.5.8 \mathrm{~b}\right]$ ]. We can find their individual conductance peaks as a function of $\phi$ by setting $\theta_{\mathrm{g}}^{(\eta, n)}=2 m \pi$ where $m \in \mathbb{Z}$. This particular $\theta_{\mathrm{g}}^{(\eta, n)}$ value causes the transmission denominator to become a minimum and its numerator to become a maximum thus resulting in a resonance point. Since the $\theta_{\mathrm{g}}^{(\eta, n)}$ value at which this happens varies depending on $\pm \eta$ flavours, we find two different resonance points, respectively. The $\phi$ positions of the conductance peaks are thus given by $\phi_{r}^{(\eta, n, m)}$ where

$$
\phi_{r}^{(\eta, n, m)}=\phi_{0}\left(m-\frac{1}{2}-\tau \frac{\delta \theta_{\mathrm{g}}^{(n)}}{2 \pi}\right) .
$$

The shape and width of the resonances can vary depending on the $\chi^{(\eta, n)}$ and thus $E_{\text {in }}$. These peaks are particularly visible when we tune the energy such that $\chi^{(\eta, n)}=p \pi$ (as mentioned before) for which

$$
G=\frac{e^{2}}{\pi \hbar}\left[\delta_{\phi, \phi_{r}^{(\eta, n, m)}}+\delta_{\phi, \phi_{r}^{(-\eta, n, m)}}\right]
$$

Notice how the peaks are symmetric around $\phi_{0} m$ and $\phi_{0}(m-1 / 2)$. For 
most other values of $E_{\text {in }}$, these peaks broaden and start to overlap and eventually result in the formation of an accumulative peak or trough at $\phi_{0} m$ or $\phi_{0}(m-1 / 2)$. This causes the two separate $\eta$ contributions to become indistinguishable.

Thus for most fixed injection energy values, there are generally resonance areas [this is explicitly shown in Fig. (5.3)] around $\phi=\phi_{0} m$ or $\phi_{0}(m-1 / 2)$. It turns out that the $\phi$ position where the resonance area occurs is determined by the sign of $\cos \left(\delta \theta_{\mathrm{g}}^{(n)}\right)$. This dependence is shown in the following derivation where we sum over the $\tau$ valley conductances using Eqns. (5.8b) and (5.11) 3 when $\phi^{(m)}=\frac{m}{2} \phi_{0}$ :

$$
\begin{aligned}
G\left(\phi^{(m)}\right) & \propto \cos ^{2}\left(\frac{\theta_{\mathrm{g}}^{(1, n)}}{2}\right)+\cos ^{2}\left(\frac{\theta_{\mathrm{g}}^{(-1, n)}}{2}\right) \\
& \propto \sin ^{2}\left(\frac{m \pi+\delta \theta_{\mathrm{g}}^{(n)}}{2}\right)+\sin ^{2}\left(\frac{m \pi-\delta \theta_{\mathrm{g}}^{(n)}}{2}\right) \\
& \propto 1-e^{i m \pi} \cos \left(\delta \theta_{\mathrm{g}}^{(n)}\right)
\end{aligned}
$$

where we are at a constant injection energy. Note that this is possible since the $\cos \left(\theta_{g}^{(1, n)}\right)=\cos \left(\theta_{g}^{(-1, n)}\right)$ value and $\chi_{\mathrm{s}}^{(\eta, n)}$ of the conductances stay constant for different $\eta$ values, as shown in Sec. (5.1). Generally, a positive value of $\cos \left(\delta \theta_{\mathrm{g}}^{(n)}\right)$ corresponds to a trough and a negative value to a peak. However as shown before, at certain injection energies $E_{\text {in }} \approx E_{\min }^{(p)}$, more peaks can appear in the interval of $2 \pi$ due to narrowing of individual $\eta$ conductance contribution's peak widths. In this case, a negative value of $\cos \left(\delta \theta_{\mathrm{g}}^{(n)}\right)$ will represent a local minima.

Eqns. (5.13) and 5.15) present us two semi-independent methods that would allow experimentalists to measure the correction to the Berry phase, $\delta \theta_{\mathrm{g}}^{(n)}$. The first method, using Eqns. 5.13 , one can determine $\delta \theta_{\mathrm{g}}^{(n)}$ by tuning through a period of $\phi$ for $\chi^{(\eta, n)} \approx p \pi$ and making note of the $\phi$ separation of the $\pm \eta$ peaks. This method is completely accurate when $\left|\delta \theta_{\mathrm{g}}^{(n)}\right|<\pi$. However, for $\left|\delta \theta_{\mathrm{g}}^{(n)}\right| \geq \pi$, there is a certain amount of ambiguity due to the

\footnotetext{
${ }^{3}$ For SLG and SQW, this calculation should be straightforward. For TMD, this proportionality rule is only valid when we purely consider the separate $\tau s_{3}$ conductances where we also sum over the $\tau= \pm 1$ valleys keeping $\tau s_{3}$ constant. This is because $\delta \theta_{\mathrm{g}}^{(n)}$ may vary for different values of $\tau s_{3}$.
} 
periodicity of the conductance as a function of magnetic flux. Fortunately, it turns out that most $\delta \theta_{\mathrm{g}}^{(n)}$ values have a magnitude that is smaller than $\pi$ which is shown definitively in Chapter 6 .

The second method can be implemented close to zero field strength where we can determine a range within which the confinement-induced Berry phase must be due to the presence of a peak or trough. Although this wouldn't give us the exact value of $\delta \theta_{\mathrm{g}}^{(n)}$, unless we already knew the exact $E_{\text {in }}$ value, it would give us a range $(2 m+1 / 2) \pi<\delta \theta_{\mathrm{g}}^{(n)}<(2 m+3 / 2) \pi$ (if a peak is present) or $(2 m-1 / 2) \pi<\delta \theta_{\mathrm{g}}^{(n)}<(2 m+1 / 2) \pi$ (if a trough is present) when $\phi=0$. Additionally, when $\delta \theta_{\mathrm{g}}^{(n)}=(2 m+1 / 2) \pi$, we have a $\phi$ periodicity of $\phi_{0} / 2$.

We now proceed to find the specific theoretical values of $\delta \theta_{\mathrm{g}}^{(n)}$ and $\chi_{\mathrm{s}}^{(\tau, n)}$ for the materials: massless-Dirac SLG, TMD and SQW rings. The form of the conductance as a function of the relevant ring parameters are then shown and discussed with reference back to results from this section. For simplicity, we define $\Delta E_{\text {in }} \equiv E_{\text {in }}-E_{\alpha}^{(\eta, n)}(0)$ henceforth.

\subsection{Massless-Dirac single-layer graphene}

For a perfect SLG QR, we can rearrange the subband dispersion [Eqn. (3.33)] for $l_{ \pm}$to find the relevant equation for the phases:

$$
\begin{aligned}
\delta \theta_{\mathrm{g}}^{(n)} & =2 \pi \frac{\frac{4}{\pi^{3}(2 n-1)^{3}} \epsilon^{2}}{\left(\frac{2}{\pi(2 n-1)} \epsilon\right)^{2}-\left(\frac{4}{\pi^{3}(2 n-1)^{3}} \epsilon^{2}\right)^{2}} \frac{E_{\mathrm{in}}}{E_{\mathrm{SLG}}^{(\tau, n)}(0)} \\
& \approx \frac{1}{\left(n-\frac{1}{2}\right)} \frac{E_{\mathrm{in}}}{E_{\mathrm{SLG}}^{(\tau, n)}(0)}
\end{aligned}
$$


and

$$
\begin{aligned}
\chi_{\mathrm{S}}^{(\tau, n)} & =\frac{\sqrt{\left(\frac{4}{\pi^{3}(2 n-1)^{3}} \epsilon^{2}\right)^{2}+\left(\frac{2}{\pi(2 n-1)} \epsilon\right)^{2}\left(\left[\frac{E_{\mathrm{in}}}{E_{\mathrm{SLG}}^{(\tau, n)}(0)}\right]^{2}-1\right)}}{\left(\frac{2}{\pi(2 n-1)} \epsilon\right)^{2}-\left(\frac{4}{\pi^{3}(2 n-1)^{3}} \epsilon^{2}\right)^{2}} \\
& \approx \pi \frac{R}{W} \frac{\sqrt{E_{\mathrm{in}}^{2}-\left[E_{\mathrm{SLG}}^{(\tau, n)}(0)\right]^{2}}}{\hbar v / W}
\end{aligned}
$$

where $E_{\mathrm{SLG}}^{(\tau, n)}(0) \equiv E_{\mathrm{SLG}, 0}^{(\tau, n)}$, as given by Eqn. $3.17 \mathrm{~b}$, and in the second line of the equations we have discarded the higher order $\epsilon \equiv W / R$ terms since $\epsilon$ 1. Interestingly, the confinement-related contribution $\delta \theta_{\mathrm{g}}^{(n)}$ to the system's geometric phase is approximately constant for a fixed device geometry, and is even a universal function of $V_{0} /(\hbar v / W)$ in the limit of small aspect ratio $W / R$. Thus a useful separation of parametric dependences occurs, where $\chi_{\mathrm{s}}^{(\tau, n)}$ varies strongly with the Fermi energy $E_{\text {in }}$ in the leads but is independent of the magnetic flux $\phi$, while $\theta_{\mathrm{g}}^{(\tau, n)}$ depends on the magnetic flux but only weakly on $E_{\text {in }}$. Studying the ring conductance as a function of both $\phi$ and $E_{\text {in }}$ will therefore allow, in principle, to extract the relevant ring-structure parameters.

Using Eqn. (5.11), we can plot the massless-Dirac SLG conductance $G$ as a function of energy difference $\Delta E_{\text {in }}, \phi$ and energy levels $n$. For the $n=1$ subband, the conductance versus injection energy for different lead transparency values can be seen in Fig. (5.2).

We see that the shape of the curves are similar to those from Ref. 35] but are warped due to the parametrisation of $\chi_{\mathrm{s}}$ in terms of energy. When $\epsilon_{c}=1 / 2$, the junction is transparent for electrons travelling from the lead to the ring (or vice versa) and thus there is a high amount of transmission as compared to the $\epsilon_{c}=1 / 4$ and $1 / 16$ cases. However, there is a zero conductance value situated at $E_{\min }^{(p)}$ that is attributable to the perfect destructive interference of the partial waves within the ring. In the cases of $\epsilon_{c}=1 / 4$ and $1 / 16$, we see that there are only strong resonances close to the $E_{\min }^{(p)}$ value. This is because as the junction becomes less transparent, the resonances are sharper and harder to tune to. 


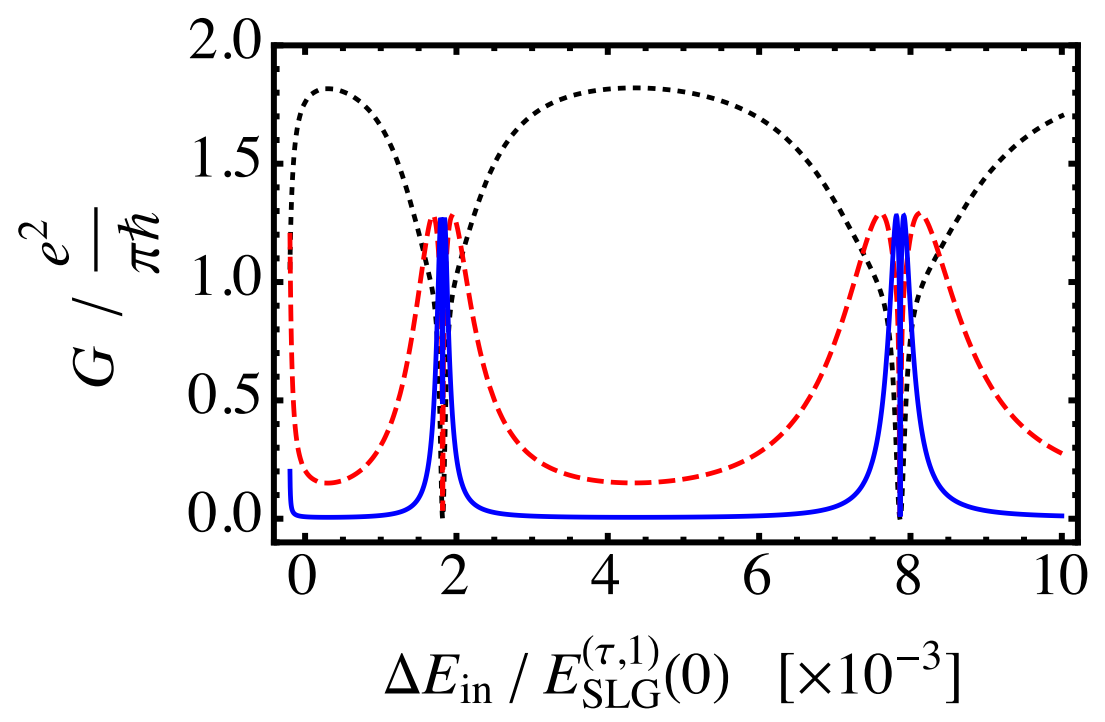

Figure 5.2: Figure of massless-Dirac SLG conductance G [Eqn. (5.11)] as a function of energy $\Delta E_{\text {in }}$ for different values of lead transparency $\epsilon_{c}=1 / 2$ (dotted black), 1/4 (dashed red) and 1/16 (solid blue). The other parameters were held constant at $W / R=0.1, n=1$ and $\phi / \phi_{0}=0.1$.

We also notice an eventual elongation of the resonance region as we increase $E_{\text {in }}$. This is due to the parametrisation of $\chi_{\mathrm{s}}$ in terms of the energy which behaves as $\chi_{\mathrm{s}} \propto \sqrt{E_{\mathrm{in}}^{2}-E_{\mathrm{SLG}}^{(\tau, n)}(0)^{2}}$. This means that as we increase $E_{\text {in }}$, the maxima become separated further apart and elongated. Additionally, there are consistently two resonance peaks (particularly visible for $\epsilon_{c}=1 / 4$ and $1 / 16)$ that appear to surround a minimum that is described by $\chi^{(\tau, p)}=p \pi$, as mentioned in the previous section. These minima, where $G$ is given by Eqn. 5.14 , can be found at energies $E_{\min }^{(p)}$ :

$$
\begin{aligned}
\frac{E_{\min }^{(p)}}{E_{\mathrm{SLG}, 0}^{(\tau, n)}} & =\sqrt{1-\left(\frac{2}{\pi^{2}(2 n-1)^{2}} \epsilon\right)^{2}} \times \\
& \sqrt{1+p^{2}\left[\left(\frac{2}{\pi(2 n-1)} \epsilon\right)^{2}-\left(\frac{4}{\pi^{3}(2 n-1)^{3}} \epsilon^{2}\right)^{2}\right]}
\end{aligned}
$$

where $p \in \mathbb{Z}$. This equation corresponds very well to the $G=0$ points in Fig. (5.2). Notice that the very first minima occurs at $p=0$ which gives

$$
\frac{E_{\min }^{(0)}}{E_{\mathrm{SLG}, 0}^{(\tau, n)}}=\sqrt{1-\left(\frac{2}{\pi^{2}(2 n-1)^{2}} \epsilon\right)^{2}} .
$$


This value has an energy $E_{\text {in }}$ that is smaller than $E_{\mathrm{SLG}, 0}^{(\tau, n)}$ and corresponds to the vertex point of the subband dispersion. Although not explicitly shown in Fig. (5.2), we can see indications of this point since the conductance rises for $\Delta E_{\text {in }}<0$ to form a maxima.

In Fig. (5.3) we display a contour plot of the conductance as a function of both injection energy and magnetic flux. We observe that, as expected, the conductance is periodic with period $\phi_{0}$. It is also visible to us that the resonance area [see definition in Fig. (5.3)] is centred around $\phi / \phi_{0}=m$ which indicates that $\left(2 m^{\prime}+1 / 2\right) \pi<\delta \theta_{\mathrm{g}}^{(n)}<\left(2 m^{\prime}+3 / 2\right) \pi$ where we have made use of Eqn. 5.15). Using our analytical expression for $\delta \theta_{\mathrm{g}}^{(n)}$, we find that this is indeed true since $\delta \theta_{\mathrm{g}}^{(1)} / \pi=2 / \pi$ which is within the range.

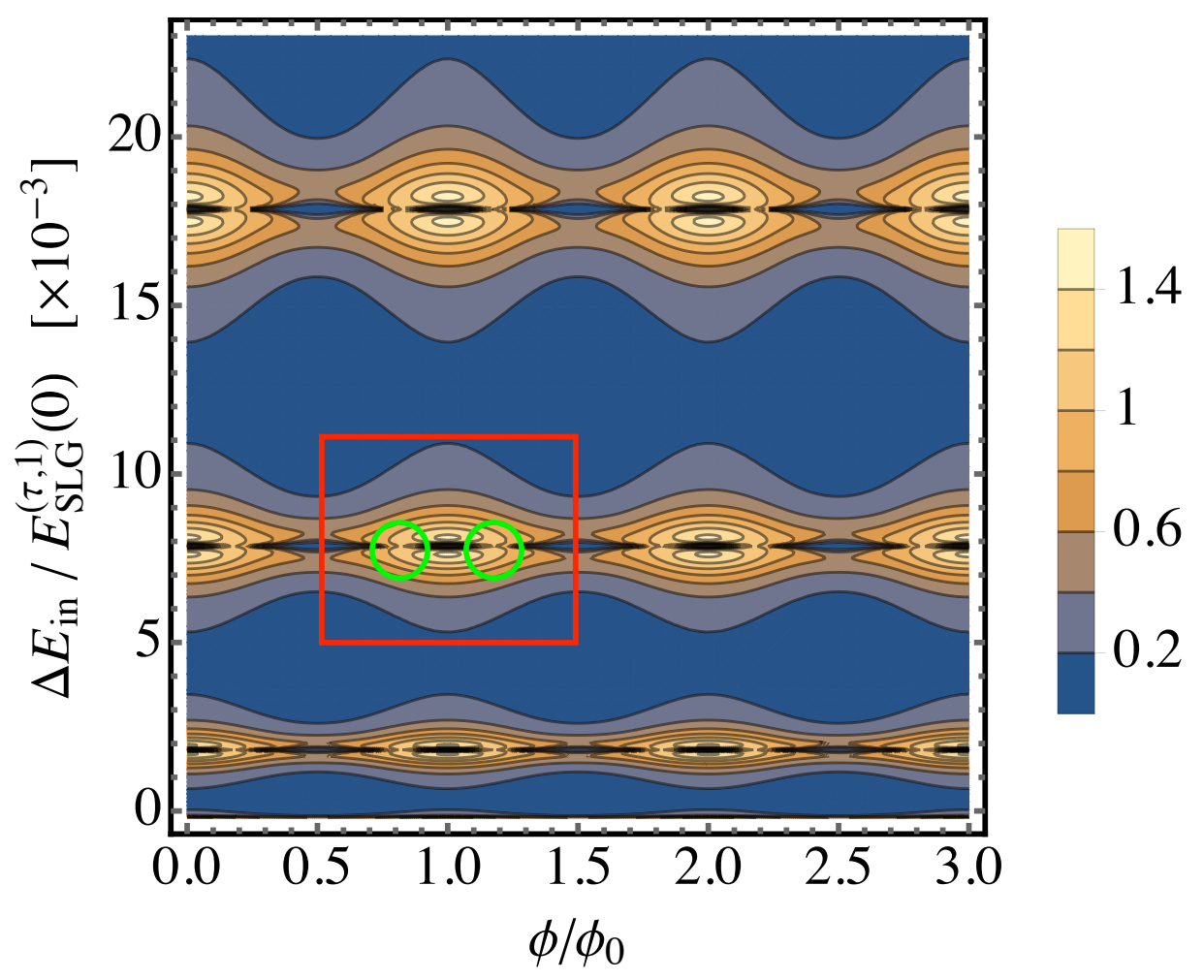

Figure 5.3: Contour-plot of massless-Dirac SLG conductance in units of $e^{2} / \pi \hbar$ as a function of $\Delta E_{\text {in }}$ versus $\phi / \phi_{0}$ for $\epsilon_{c}=1 / 4, W / R=0.1$ and $n=1$. We see the persistent periodicity of $G$ as a function of $\phi / \phi_{0}$. What we name the "resonance area" is indicated by the red box which is currently centred around $\phi / \phi_{0}=m$ but may shift to $\phi / \phi_{0}=m-1 / 2$ depending on system parameters. The two separate $\tau$ valley conduction peaks that occurs in this area are circled in green [also shown in Fig. (5.4)].

Additionally, if we take a look at the $\phi$ dependence of the figure at the 
energies close to $E_{\mathrm{min}}^{(p)}$, we see the predicted two valley splitting that becomes apparent (in the figure, we have circled samples of these peaks in green). For $E_{\text {in }} \approx E_{\text {min }}^{(1)}$, we plot this dependence in Fig. 5.4 . The difference between the $\tau$ valleys conductance peaks is equivalent to $\delta \theta_{\mathrm{g}}^{(n)} / \pi$, as anticipated from Eqn. (5.13), which should allow experimentalists to determine $\delta \theta_{\mathrm{g}}^{(n)}$.

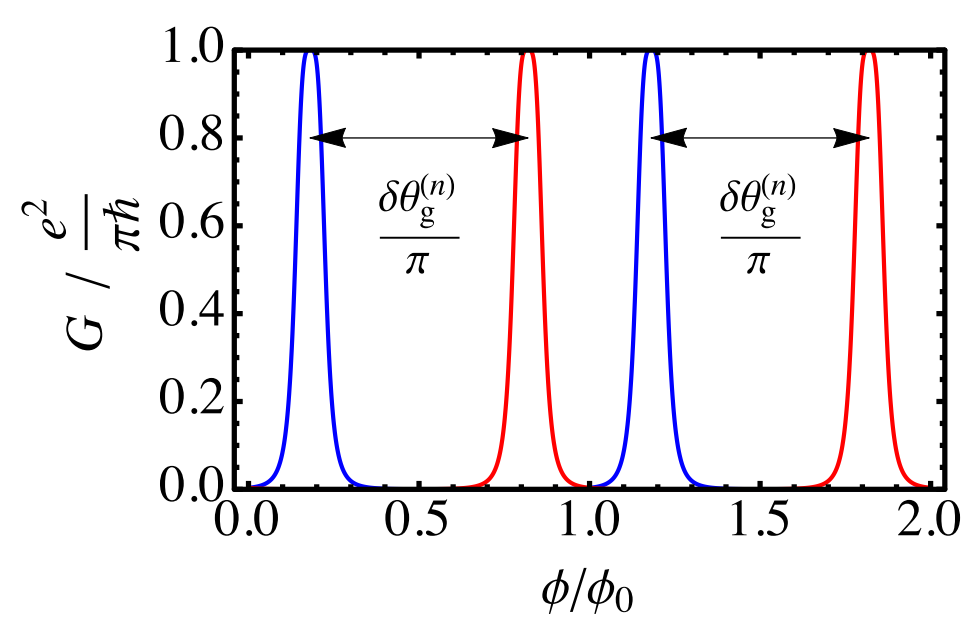

Figure 5.4: Lowest-subband $\mathbf{K}$ (blue) and $\mathbf{K}^{\prime}$ (red) valley massless-Dirac $S L G$ conductances as a function of $\phi$ for a fixed $\Delta E_{\mathrm{in}} / E_{\mathrm{SLG}}^{(\tau, 1)}(0)=1.82 \times 10^{-3}$. Since our energy is close to $E_{\min }^{(1)}$, we are able to see the distinct conductances corresponding to different valley contributions. Other parameter are held constant at $\epsilon_{c}=1 / 4, W / R=0.1$.

We now proceed to investigate the explicit forms of the phase and conductance for TMD QRs. Since the general TMD subband dispersion, as given by Eqn. 3.45, can be easily modified to describe a general SLG by setting $\lambda=0$, we only explicitly find $\delta \theta_{\mathrm{g}}^{(n)}$ and $\chi_{\mathrm{s}}^{(\tau, n)}$ for the TMD QR.

\subsection{Transition-metal dichalcogenide}

For TMD the values of $\delta \theta_{\mathrm{g}}^{(n)}$ and $\chi_{\mathrm{s}}^{(\tau, n)}$ can be found by inverting the subband dispersion [Eqn. (3.45)] for the $l_{ \pm}^{(\tau, n)}$ values. The phases are given by

$$
\begin{aligned}
\delta \theta_{\mathrm{g}}^{(n)} & =2 \pi \frac{\Gamma_{\mathrm{TMD}}}{1-\left(\frac{W}{R}\right)^{2} \Gamma_{\mathrm{TMD}}^{2}}\left[E_{\mathrm{in}}-\frac{\lambda}{2} \tau s_{3}\right] \frac{W}{\hbar v} \\
& \approx 2 \pi \Gamma_{\mathrm{TMD}}\left[E_{\mathrm{in}}-\frac{\lambda}{2} \tau s_{3}\right] \frac{W}{\hbar v}
\end{aligned}
$$


and

$$
\begin{aligned}
\chi_{\mathrm{s}}^{(\tau, n)} & =\pi \frac{W}{\hbar v} \frac{R}{W} \frac{\sqrt{\left[\left(\frac{W}{R}\right)^{2} \Gamma_{\mathrm{TMD}}^{2}-1\right]\left[E_{\mathrm{TMD}, 0}^{\left(\tau s_{3}, n\right)}\right]^{2}+\left[E_{\mathrm{in}}-\frac{\lambda}{2} \tau s_{3}\right]^{2}}}{1-\left(\frac{W}{R}\right)^{2} \Gamma_{\mathrm{TMD}}^{2}} \\
& \approx \pi \frac{R}{W} \frac{\sqrt{\left[E_{\mathrm{in}}-\frac{\lambda}{2} \tau s_{3}\right]^{2}-\left[E_{\mathrm{TMD}, 0}^{\left(\tau s_{3}, n\right)}\right]^{2}}}{\hbar v / W}
\end{aligned}
$$

where we have applied the same simplification, in the second line, as before with the massless-Dirac SLG QR. As in the massless-Dirac SLG case, $\delta \theta_{\mathrm{g}}^{(n)}$ is approximately a constant for small changes in $E_{\text {in }}$ and can thus be considered only dependent on $\phi$. We also see that $\chi_{\mathrm{s}}^{(\tau, n)}$ is strongly dependent on $E_{\text {in }}$ with zero dependence on $\phi$.

For a $\mathrm{MoSe}_{2} \mathrm{QR}$, we can see the resultant total (both flavours $\tau s_{3}= \pm 1$ ) conductance for the $n=1$ subbands as a function of energy $E_{\mathrm{in}} / E_{\mathrm{TMD}}^{\left(\tau s_{3}=-1,1\right)}$ in Fig. (5.5). In this case, due to the small amount of spin-splitting in the conductance band, we see a superposition of two conductance patterns due to the different contribution from $\tau s_{3}= \pm 1$. Since the $\tau s_{3}=-1$ subband sits at a slightly higher energy than the $\tau s_{3}=1$ subband, as seen in Fig. (3.5), we parametrise the curve using $E_{\mathrm{TMD}}^{\left(\tau s_{3}=-1,1\right)}$ and do not look at the pure $\tau s_{3}=1$ subband conductance.

For TMDs, there exist local minima when $\chi_{\mathrm{s}}^{(p)}=p \pi$ which are situated in between resonances. Additionally, they depend now explicitly on the flavour $\tau s_{3}$, unlike in the SLG example. The energies at which these can be found at are given by

$$
\begin{array}{r}
\frac{E_{\min }^{(p)}}{E_{\mathrm{TMD}, 0}^{\left(\tau s_{3}, n\right)}}=\sqrt{1-\left(\frac{W}{R}\right)^{2} \Gamma_{\mathrm{TMD}}^{2}} \sqrt{1+\left(\frac{\hbar v p}{E_{\mathrm{TMD}, 0}^{\left(\tau s_{3}, n\right)} R}\right)^{2}\left[1-\left(\frac{W}{R}\right)^{2} \Gamma_{\mathrm{TMD}}^{2}\right]} \\
+\frac{\frac{\lambda}{2} \tau s_{3}}{E_{\mathrm{TMD}, 0}^{\left(\tau s_{3}, n\right)}}
\end{array}
$$

In Fig. (5.6) we depict the contour plot of the total and individual $\tau s_{3}$ contributions to the conductance as a function of energy and magnetic flux 


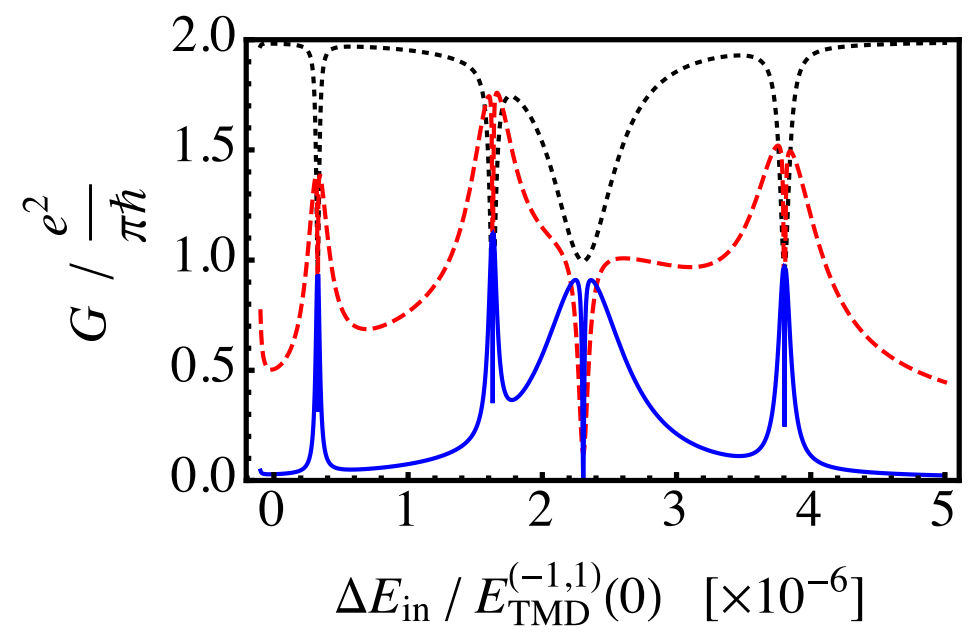

Figure 5.5: Figure of $\mathrm{MoSe}_{2} n=1$ subband conductance $G$ as a function of energy $E_{\mathrm{TMD}}^{(1)}(l)$ for different values of lead transparency $\epsilon_{c}=1 / 2$ (dotted black), 1/4 (dashed red) and 1/16 (solid blue). The other parameters were held constant at $\lambda=-90 \mathrm{meV}, \hbar v=253 \mathrm{meV} \mathrm{nm}$ and $\Delta=1.40 \mathrm{eV}, W=$ $40 \mathrm{~nm}, W / R=0.1, n=1$ and $\phi / \phi_{0}=0.1$.

for $\mathrm{MoSe}_{2}$ QRs. We see that the $\tau s_{3}=1$ resonance point is significantly elongated compared to those of $\tau s_{3}=-1$. This is because the larger the energy difference is between $E_{\text {in }}$ and $E_{\mathrm{TMD}}^{\left(\tau s_{3}, n\right)}(0)$, the more elongated the conductance shapes will be due to the parametrisation of $\chi_{\mathrm{s}}$ which is $\propto \sqrt{E_{\mathrm{in}}^{2}-E_{\mathrm{TMD}}^{\left(\tau s_{3}, n\right)}(0)^{2}}$. Since $E_{\mathrm{TMD}}^{(1,1)}(0)$ is below $E_{\mathrm{TMD}}^{(-1,1)}(0)$, this means that the energy difference between $E_{\text {in }}$ and $E_{\mathrm{TMD}}^{(1,1)}(0)$ is larger and thus results in the longer stretched patterns.

We see that the shape of the resonances, as a function of $\phi$, differ greatly from those of the massless-Dirac SLG case since the $\phi$ dependence of $\left(\tau, s_{3}\right)=$ $\mathbf{\pm}(1,1)$ [see Fig. 5.6a)] and $\mathbf{\pm}(1,-1)$ [see Fig. (5.6b)] flavour conductions do not exemplify two distinct $\phi_{r}^{( \pm \eta, n, m)}$ flavour peaks at $E_{\text {in }}=E_{\min }^{(p)}$ [i.e. the two green circles in Fig. (5.3) are not distinguishable for this case]. Since the maxima seem to only occur at $\phi / \phi_{0}=m$, this indicates that $\delta \theta_{\mathrm{g}}^{(n)} \approx m \pi$. If we calculate, using Eqn. 5.23 , the actual value of $\delta \theta_{\mathrm{g}}^{(n)}$ for a $\mathrm{MoSe}_{2}$ ring we find the value to be $0.999 \pi$ for $\left(\tau, s_{3}\right)= \pm(1,1)$ and $0.998 \pi$ for $\mathbf{\pm}(1,-1)$.

The total conductance for a $\mathrm{MoSe}_{2}$ ring can be seen in Fig. 5.6c) which once again shows the superposition of the $\tau s_{3}$ flavours. Since the $\delta \theta_{\mathrm{g}}^{(n)}$ values are very similar for both flavours, we see that the resonances align along the same $\phi / \phi_{0}=m$ values. In a hypothetical situation where the Berry phases 


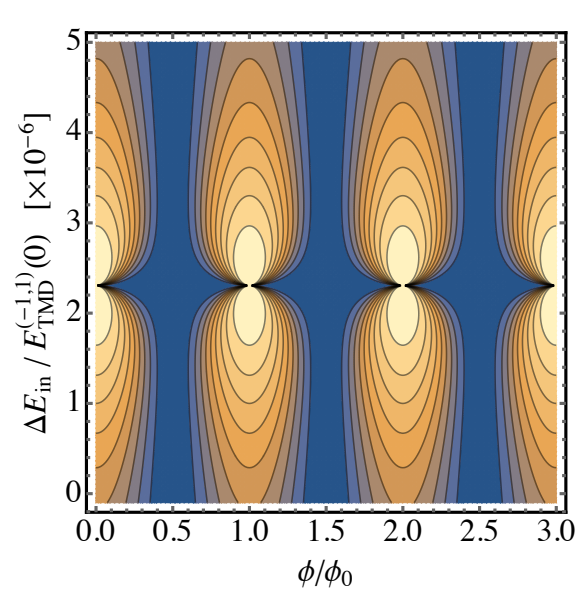

(a) $\tau s_{3}=1$ conductance

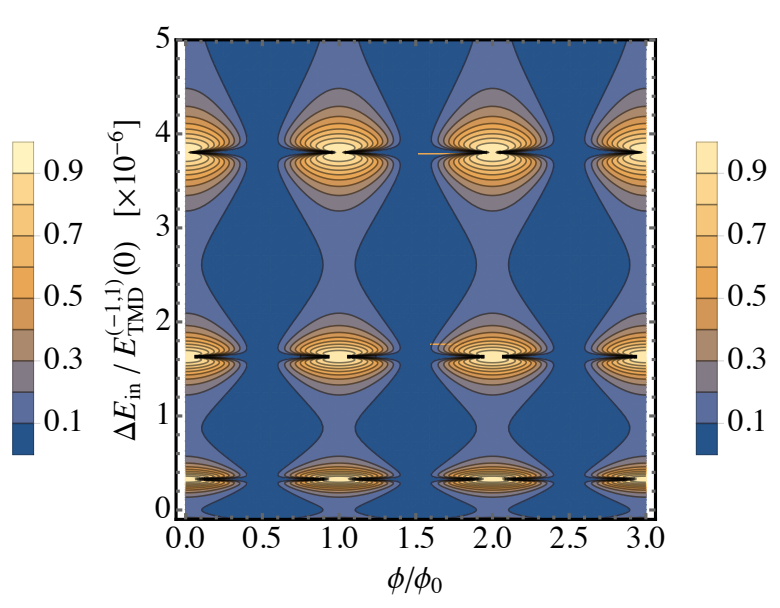

(b) $\tau s_{3}=-1$ conductance

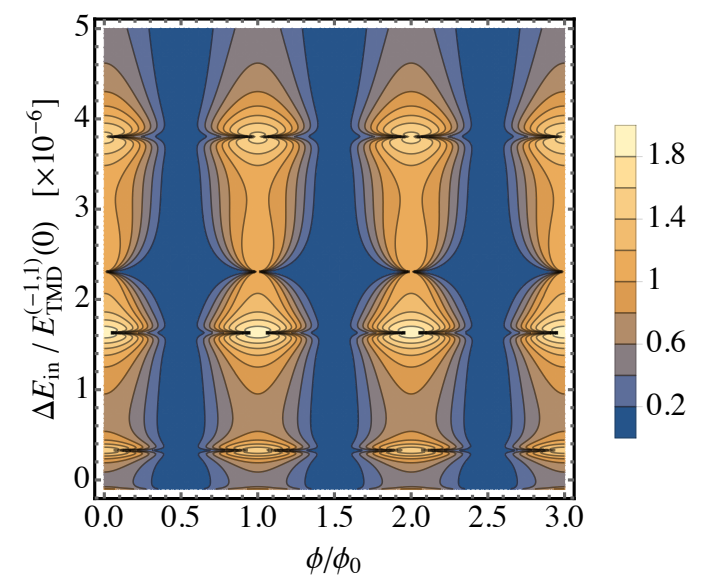

(c) Total conductance of $n=1$ states

Figure 5.6: Contour-plot of conductance of $\mathrm{MoSe}_{2} n=1$ subband states in units of $e^{2} / \pi \hbar$ as a function of $E_{\mathrm{TMD}}^{(1)}(l)$ versus $\phi / \phi_{0}$. (a) Conductance with only the $\tau s_{3}=1$ lowest-subband states. (b) Conductance with only the $\tau s_{3}=-1$ lowest-subband states. (c) Total conductance of the lowestsubband states. We see the persistent periodicity of $G$ as a function of $\phi / \phi_{0}$. Other parameters were held constant at $\epsilon_{c}=1 / 4, W=40 \mathrm{~nm}, W / R=0.1$, $\Delta=1400 \mathrm{meV}, \lambda=-90 \mathrm{meV}, \hbar v=253 \mathrm{meV} \mathrm{nm} \mathrm{and} n=1$. 
were visibly different for the two flavours, this would no longer hold, thus resulting in an even further complicated conductance pattern.

We have now finished our analysis of the TMD QR conductance and move on to understanding the more complex system of SQW QRs. It is difficult to derive a full analytical solution for the phases and conductances and thus we only present a simplified analytical solution where we neglect diagonal $l^{2}$ terms in $H_{\mathrm{SQW}, 1}^{(\tau)}$. This will hopefully allow us to gain some insight into the simplified system which will guide our understanding for the general system. After this, the general result for the conductances will be explored using numerical calculations.

\subsection{Semiconductor quantum well}

Rearranging Eqn. 4.48 for $l_{ \pm}^{(\tau, n)}$ will result in four solutions, of which two will be real and two imaginary due to the additional presence of the $l^{2}$ terms in $H_{\mathrm{SQW}, 1}^{(\tau)}$. We simplify the equation such that we can find analytical solutions for $\theta_{\mathrm{g}}^{(\tau, n)}$ and $\chi_{\mathrm{s}}^{(\tau, n)}$ using an approximation that the terms associated with the $l^{2}$ term are negligible for small $l$, large $\tilde{W}$ and small $\xi_{\mathrm{M}}$ (i.e. $H_{\mathrm{SQW}, 1}^{(\tau, n)} \approx$ $\left.\hbar v l / r \sigma_{2}\right)$. Using this method, the $\delta \theta_{\mathrm{g}}^{(n)}$ values are given by

$$
\begin{aligned}
\delta \theta_{\mathrm{g}}^{(n)} & =\pi\left[\frac{\Lambda_{\mathrm{SQW}}+\Lambda_{\mathrm{SQW}}^{\prime}}{\left|\Delta_{\mathrm{SQW}}\right|^{2}-\left(\frac{\tilde{W}}{\tilde{R}}\right)^{2} \Lambda_{\mathrm{SQW}} \Lambda_{\mathrm{SQW}}^{\prime}} \tilde{E}_{\mathrm{in}}-\frac{\Lambda_{\mathrm{SQW}}^{\prime} \tilde{E}_{\mathrm{SQW}, 0}^{(\tau, n)}+\Lambda_{\mathrm{SQW}} \tilde{E}_{\mathrm{SQW}, 0}^{(\tau,-n)}}{\left|\Delta_{\mathrm{SQW}}\right|^{2}-\left(\frac{\tilde{W}}{\tilde{R}}\right)^{2} \Lambda_{\mathrm{SQW}} \Lambda_{\mathrm{SQW}}^{\prime}}\right] \\
& \approx \frac{\pi}{\left|\Delta_{\mathrm{SQW}}\right|^{2}}\left[\Gamma_{\mathrm{SQW}}\left(\tilde{E}_{\mathrm{in}}-\tilde{E}_{\mathrm{SQW}, 0}^{(\tau,-n)}\right)+\Gamma_{\mathrm{SQW}}^{\prime}\left(\tilde{E}_{\mathrm{in}}-\tilde{E}_{\mathrm{SQW}, 0}^{(\tau, n)}\right)\right]
\end{aligned}
$$


and

$$
\begin{aligned}
& \chi_{\mathrm{s}}^{(\tau, n)}=\frac{\pi}{\left|\Delta_{\mathrm{SQW}}\right|^{2}-\left(\frac{\tilde{W}}{\tilde{R}}\right)^{2} \Lambda_{\mathrm{SQW}} \Lambda_{\mathrm{SQW}}^{\prime}} \times \\
&\left(\left[\left|\Delta_{\mathrm{SQW}}\right|^{2}-\left(\frac{\tilde{W}}{\tilde{R}}\right)^{2} \Lambda_{\mathrm{SQW}} \Lambda_{\mathrm{SQW}}^{\prime}\right]\left(\tilde{E}_{\mathrm{in}}-\tilde{E}_{\mathrm{SQW}, 0}^{(\tau, n)}\right)\left(\tilde{E}_{\mathrm{in}}-\tilde{E}_{\mathrm{SQW}, 0}^{(\tau,-n)}\right)\right. \\
&\left.+\left(\frac{\tilde{W}}{\tilde{R}}\right)^{2}\left[\frac{\Lambda_{\mathrm{SQW}}^{\prime}\left(\tilde{E}_{\mathrm{in}}-\tilde{E}_{\mathrm{SQW}, 0}^{(\tau, n)}\right)+\Lambda_{\mathrm{SQW}}\left(\tilde{E}_{\mathrm{in}}-\tilde{E}_{\mathrm{SQW}, 0}^{(\tau,-n)}\right)}{2}\right]^{2}\right)^{\frac{1}{2}} \\
& \approx \\
& \frac{\pi}{\left|\Delta_{\mathrm{SQW}}\right|} \sqrt{\left(\tilde{E}_{\mathrm{in}}-\tilde{E}_{\mathrm{SQW}, 0}^{(\tau, n)}\right)\left(\tilde{E}_{\mathrm{in}}-\tilde{E}_{\mathrm{SQW}, 0}^{(\tau,-n)}\right)}
\end{aligned}
$$

where we have simplified the second lines of the equations by neglecting larger orders of $\epsilon=W / R$.

The Berry phase can be further simplified for certain injection energy regimes. In particular, a realistic simplification involves setting the injection energy to approximately the $l=0$ energy of the subband dispersion, i.e. $E_{\mathrm{in}} \approx \tilde{E}_{\mathrm{SQW}, 0}^{(\tau, \pm n)}$, thus resulting in the equations:

$$
\delta \theta_{\mathrm{g}}^{(n)} \approx \begin{cases}\pi \frac{\Lambda_{\mathrm{SQW}}}{\left|\Delta_{\mathrm{SQW}}\right|^{2}}\left[\tilde{E}_{\mathrm{SQW}, 0}^{(\tau, n)}-\tilde{E}_{\mathrm{SQW}, 0}^{(\tau,-n)}\right] & \text { for } E_{\mathrm{in}} \approx \tilde{E}_{\mathrm{SQW}, 0}^{(\tau, n)},(5.3 \\ \pi \frac{\Lambda_{\mathrm{SQW}}^{\prime}}{\left|\Delta_{\mathrm{SQW}}\right|^{2}}\left[\tilde{E}_{\mathrm{SQW}, 0}^{(\tau,-n)}-\tilde{E}_{\mathrm{SQW}, 0}^{(\tau, n)}\right] & \text { for } E_{\mathrm{in}} \approx \tilde{E}_{\mathrm{SQW}, 0}^{(\tau,-n)} \cdot(5,\end{cases}
$$

These forms of the $\delta \theta_{\mathrm{g}}^{(n)}$ and $\chi_{\mathrm{s}}^{(\tau, n)}$ are very similar to those of SLG and TMD. However, one obvious difference is the lack of energy-reflection symmetry thus leading to separate equations that depend on the type of the state [either (a) or (b)]. Once again, for a fixed device geometry, we see that $\delta \theta_{\mathrm{g}}^{(n)}$ is essentially a constant at the energies that we consider and thus is dominated by the change of $\phi . \chi_{\mathrm{s}}^{(\tau, n)}$ is once again strongly dependent on the injection energy and completely independent of the magnetic flux.

Although this is just a simplified model, we see that $\delta \theta_{\mathrm{g}}^{(n)}$ is essentially directly proportional to $\Lambda_{\mathrm{SQW}}$ and $\Lambda_{\mathrm{SQW}}^{\prime}$ for the two different types, respectively. Fortunately this particular property also remains true even when we 
include the $l^{2}$ terms in the Hamiltonian which we will discover in the Chapter 6. It also turns out that when we include the $l^{2}$ terms, $\chi_{\mathrm{s}}^{(\tau, n)}$ also stays proportional to $\sqrt{\left(\tilde{E}_{\mathrm{in}}-\tilde{E}_{\mathrm{SQW}, 0}^{(\tau, n)}\right)\left(\tilde{E}_{\mathrm{in}}-\tilde{E}_{\mathrm{SQW}, 0}^{(\tau,-n)}\right)}$ which causes the elongation of resonance areas as a function of $E_{\text {in }}$, which were seen in the SLG and TMD cases.

Using our simplified analytical model and comparing it to the numerical and generalised analytical (this will be shown in Chapter 6) solutions for the complete system allows to gain insight into the effects of the $l^{2} / r^{2}$ terms that arises in $H_{\mathrm{SQW}, 1}^{(\tau)}$. We will come back to this comparison in the next chapter in which the difference of the Berry phase with and without the $l^{2}$ terms will be substantial for large $\xi_{\mathrm{M}}$.

We now focus our attention upon the conductance of the complete system (i.e. including the $l^{2}$ terms) which we can calculate using a numerical method ${ }^{4}$. In Fig. (5.7), we plot the conductance as a function of injection energy and magnetic flux in the cases of $\xi_{\mathrm{M}}<\xi_{\mathrm{Mc} \pm}$ and $\xi_{\mathrm{M}}>\xi_{\mathrm{Mc} \pm}$. Recall that $\xi_{\mathrm{Mc} \pm}$ indicates the gap values at which bound states traverse between the bulk and gap as described in Sec. 4.1.2). Ideally, we would hope to see a change in the conductance, as the gap size becomes less and less inverted [in Fig. (5.7): $\left.\xi_{\mathrm{M}} q_{0} W:-7 \rightarrow 0\right]$, that parallels the altered topological nature of these bound states as their energies transition from being inside to being outside of the gap.

In Fig. (5.7a), we have plotted the $\xi_{\mathrm{M}}<\xi_{\mathrm{Mc} \pm}$ limit where the SQW QR is in the deeply inverted regime $\left[\xi_{\mathrm{M}} q_{0} W=-7\right]$. In this case, we see that the resonance regions of conductance are centred around $\phi / \phi_{0}=m-1 / 2$ and are thus shifted by $\pm 1 / 2$ along the $\phi$ axis as compared to the SLG and TMD cases which can be seen in Figs. (5.3) and (5.6c), respectively. This is governed by the $\cos \left(\delta \theta_{\mathrm{g}}^{(n)}\right)$ component in Eqn. 5.15 which is positive [i.e. $\left.(2 m-1 / 2) \pi<\delta \theta_{\mathrm{g}}^{(n)}<(2 m+1 / 2) \pi\right]$ hence resulting in a minima at $\phi=m \phi_{0}$. Additionally, one can deduce from the figure that $\delta \theta_{\mathrm{g}}^{(n)}$ must be close to $2 m \pi$ since the $\tau$ peaks are not very distinguishable. As mentioned in Sec. (5.1.1), the exact value of $\delta \theta_{\mathrm{g}}^{(n)}$ is impossible to deduce from the figure alone due to the periodicity in $\phi$. In this case, our numerical value for $\delta \theta_{\mathrm{g}}^{(n)}$ is $0.09 \pi$ which

\footnotetext{
${ }^{4}$ For specific configurations of the system, we find the $l_{ \pm}$values by using a root finder in Mathematica.
} 


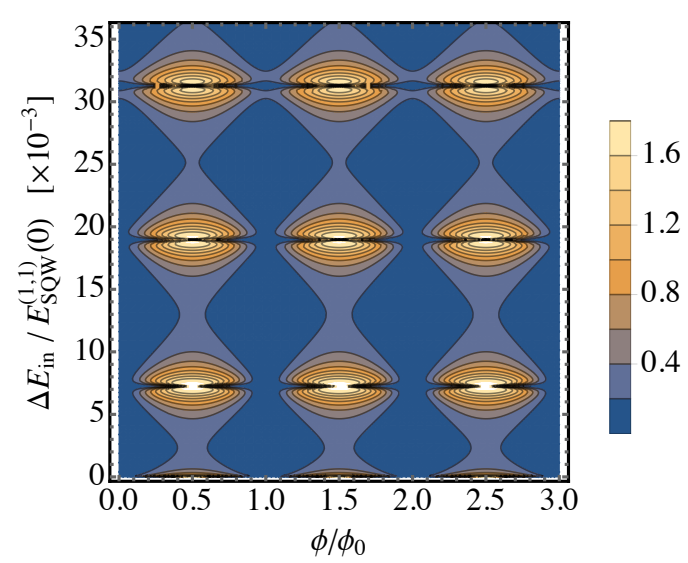

(a) $\xi_{\mathrm{M}} q_{0} W=-7$

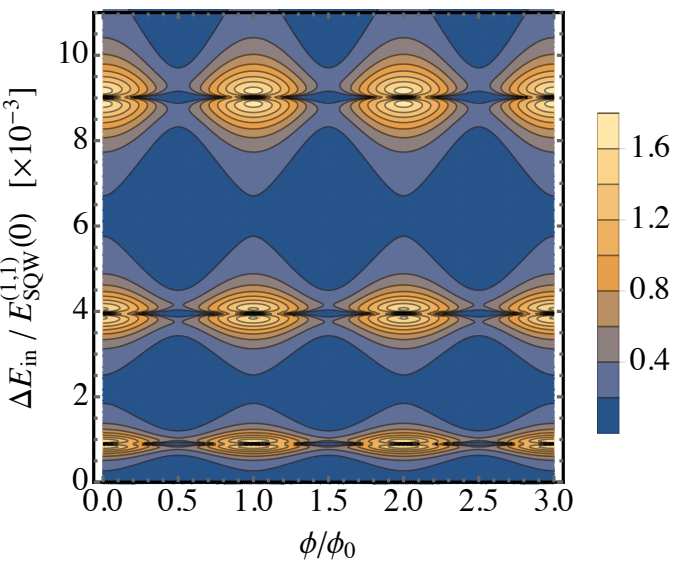

(b) $\xi_{\mathrm{M}} q_{0} W=0$

Figure 5.7: Contour-plots of conductance of $S Q W n=1$ subband state as a function of $\Delta E_{\text {in }}$ and $\phi / \phi_{0}$ for different numbers of bound states. (a) Two topological bound-state within $\pm\left|\xi_{\mathrm{M}}\right|$ for $\xi_{\mathrm{M}} q_{0} W=-7$. (b) Zero topological bound-states within $\pm\left|\xi_{M}\right|$ for $\xi_{\mathrm{M}} q_{0} W=0$. Other parameters were held constant at $n=1, \epsilon_{c}=1 / 4, \tilde{D}=-0.746, \tilde{W}=26.6, \tilde{W} / \tilde{R}=0.1$ and $\phi / \phi_{0}=0.1$.

is an extremely small value of $\delta \theta_{\mathrm{g}}^{(n)}$ as compared to the massless-Dirac SLG and TMD systems.

In Fig. $5.7 \mathrm{~b}$, we have plotted the $\xi_{\mathrm{M}}>\xi_{\mathrm{Mc} \pm}$ scenario where the system no longer possesses an inverted gap $\left[\xi_{\mathrm{M}} q_{0} W=0\right]$. We notice that the resonance regions are once again centred around $\phi / \phi_{0}=m$ which results in a significantly different conductance pattern as compared to Fig. (5.7a). This is due to the $\delta \theta_{\mathrm{g}}^{(n)}$ component that changes for a different $\xi_{\mathrm{M}}$ - the component becomes closer to $m \pi$ for Fig. $5.7 \mathrm{~b}$ and thus the $\cos \left(\delta \theta_{\mathrm{g}}^{(n)}\right)$ component becomes negative thus resulting in the resonances at $\phi / \phi_{0}=m$. This is one of the changes that indicate of the role of a changing band-gap which we will explore further in the next chapter.

We also show in Fig. (5.8) how the different subband's $\tau$ flavour conduction peaks behave as we change the gap size. This is done by setting $E_{\mathrm{in}} \approx E_{\min }^{(p)} 5$ and observing numerically how the $\tau$ flavour conduction moves.

In Fig. (5.8a), we follow the $n=1$ subband which shifts from $\delta \theta_{\mathrm{g}}^{(n)} \rightarrow 0$ in the deeply inverted regime to a much larger value that approaches $\pi$ in

\footnotetext{
${ }^{5}$ Naturally, the value of $E_{\mathrm{min}}^{(p)}$ changes as we change the gap size. However, we can just tune our energy to compensate for this.
} 


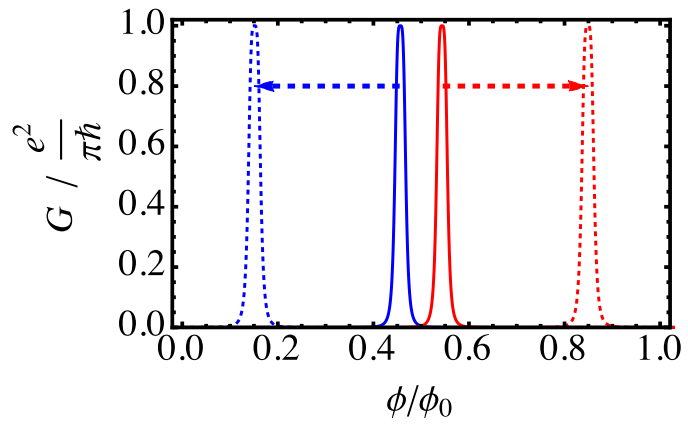

(a) $n=1$ conduction

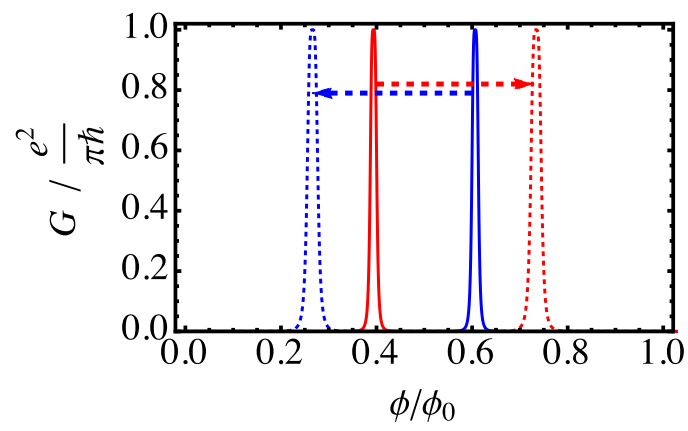

(b) $n=2$ conduction

Figure 5.8: Figures of $S Q W Q R n=1$ (a) and 2 (b) subband $\tau$ flavour conductance as a function of $\phi / \phi_{0}$ for a varying gap size $\xi_{\mathrm{M}} q_{0} W:-7$ (solid) $\rightarrow$ 0 (dotted). The flavours are depicted as $\tau=1$ (blue) and -1 (red) and the arrow indicates the movement as $\xi_{\mathrm{M}} q_{0} W:-7 \rightarrow 0$. This is done via numerical calculations in Mathematica. (a) For $n=1$, there is no swapping of the position of the flavour conductances at $\phi / \phi_{0}=m+1 / 2$. (b) For $n=2$, we see a swapping point at $\phi / \phi_{0}=m-1 / 2$ which once again indicates a behavioural difference between $n= \pm 1$ and higher subbands. Other parameters were held constant at $E_{\mathrm{in}} \approx E_{\min }^{(p)}, \epsilon_{c}=1 / 4, \tilde{D}=-0.746, \tilde{W}=26.6$ and $\tilde{W} / \tilde{R}=0.1$.

the non-inverted regime. However, we see at no point that the $\mathbf{\pm} \tau$ flavours swap positions around $\phi / \phi_{0}=m-1 / 2$ resulting in a negative value of $\delta \theta_{\mathrm{g}}^{(n)}$. We find that as we go further into the deeply inverted regime $\delta \theta_{\mathrm{g}}^{(n)} \rightarrow 0$ since the conductance peak start to overlap at $\phi / \phi_{0}=m-1 / 2$, but never swap relative positions. Naturally these discoveries parallel those described in Sec. 4.2.2 since $\delta \theta_{\mathrm{g}}^{(n)}$ is basically equivalent to the shift of the $\tau$ valley energy dispersions.

To have a direct analogy to Sec. 4.2.2), we also plot Fig. (5.8b where we study the positions of the $\tau$ valley conductances for the $n=2$ subband. Recall in Sec. 4.2.2 we discovered that the dispersions swap positions resulting due to a zero $\Lambda_{\mathrm{SQW}}^{\prime}$ value being present. We see the same pattern in $\delta \theta_{\mathrm{g}}^{(n)}$ as we change the gap parameter. Eventually, there is a gap value at which $\delta \theta_{\mathrm{g}}^{(n)}=0$ (i.e. conductances overlap at $\phi / \phi_{0}=m-1 / 2$ ) which separates the $\delta \theta_{\mathrm{g}}^{(n)}<0$ and $\delta \theta_{\mathrm{g}}^{(n)}>0$ regimes.

Now that we have seen the crucial connection between the conductance, the confinement-induced Berry phase and the $\tau$ flavour dispersion swapping, as discussed in the SLG and SQW chapters, we can cement our understanding 
of transport properties in QR systems in the next chapter. We proceed to do a detailed study of the Berry phase values for varying system parameters and set out to tie all the results together. In particular, we aim to understand the significance of SQW phases in comparison to the simpler Dirac system. 


\section{Chapter 6}

\section{Geometric Berry phase}

In the last chapter we saw that the correction to the Berry phase, $\delta \theta_{\mathrm{g}}^{(n)}$, played an integral role in the structure of the conductance and the swapping of the $\tau= \pm 1$ flavours of conduction peaks. In this chapter, the significance of this phase is explored in further detail with regards to changing system parameters, such as the gap size and ring width. The transition between the topological to non-topological regimes and its connection to $\delta \theta_{\mathrm{g}}^{(n)}$ is also examined for which we discover hallmarks of such a transition in our materials. We proceed by first investigating the form of $\delta \theta_{\mathrm{g}}^{(n)}$ for different subbands in a general SLG ring (i.e. variable gap size). Once we have a comprehensive knowledge of this relatively simple system, we use this to compare with the more complex Berry phase of the energy-reflection symmetric SQW ring, where $\tilde{D}=0$. We also draw comparisons between the $\delta \theta_{\mathrm{g}}^{(n)}$ values of the SQW system with and without the $l^{2}$ term in $H_{\mathrm{SQW}, 1}^{(\tau)}$. Using our insight of the energy-reflection symmetric systems, we finally tackle the $\tilde{D} \sigma 0$, nonenergy reflection symmetric case of SQW QRs. During this whole procedure we continuously draw parallels with the results from previous chapters to create a comprehensive summary of transport properties in 2D material QRs.

\subsection{Single-layer graphene}

Adapting from the TMD QR form of $\delta \theta_{\mathrm{g}}^{(n)}$, as given in Eqn. 5.23, we can determine an approximate-universal Berry phase for a general SLG QR system 
to be of the form:

$$
\begin{aligned}
\delta \theta_{\mathrm{g}}^{(n)} & =2 \pi \frac{\Gamma_{\mathrm{SLG}}}{1-\left(\frac{W}{R}\right)^{2} \Gamma_{\mathrm{SLG}}^{2}} \frac{E_{\mathrm{in}}}{\hbar v / W} \\
& \approx 2 \pi \Gamma_{\mathrm{SLG}} \frac{E_{\mathrm{in}}}{\hbar v / W}
\end{aligned}
$$

where we have neglected the $(W / R)^{2} \quad 1$ term in the second line. Notice how this result is "universal", i.e. solely dependent on $V_{0}$ and the confinement energy $\hbar v / W$, for a given value of $E_{\text {in }}$, since $\Gamma_{\mathrm{SLG}}$ is also completely determined by $V_{0}$ and $\hbar v / W$, as explained in the Sec. (3.1.1). Note that $\delta \theta_{\mathrm{g}}^{(n)}=-\delta \theta_{\mathrm{g}}^{(-n)}$ due to the presence of energy-reflection symmetry in SLG QR systems. We can take Eqn. (6.1) one step further by acknowledging that the injection energy is approximately equal to the size-quantisation energy: $E_{\text {in }} \approx E_{\mathrm{SLG}, 0}^{(\tau, n)}$, since we only consider a limited range of azimuthal motion $|l| \lesssim 50$. Thus, for injection energies that are close to $E_{\mathrm{SLG}, 0}^{(\tau, n)}$, we have

$$
\delta \theta_{\mathrm{g}}^{(n)} \approx 2 \pi \Gamma_{\mathrm{SLG}} \frac{E_{\mathrm{SLG}, 0}^{(\tau, n)}}{\hbar v / W}
$$

The form of $\delta \theta_{\mathrm{g}}^{(n)}$, as described by Eqn. 6.2), for the three lowest subbands $(n=1,2,3)$ can be seen in Fig. 6.1). We can notice immediately that the Berry phase of the lowest subband is drastically different from those of the higher subbands.

Firstly, in the limit $V_{0} W / \hbar v \rightarrow-\infty$ (infinitely deep inversion), the lowestsubband has $\delta \theta_{\mathrm{g}}^{(1)} \rightarrow 0$ while in higher subbands $\delta \theta_{\mathrm{g}}^{(n>1)} \rightarrow-1$. This is mainly because $E_{\mathrm{SLG}, 0}^{(\tau, 1)} \rightarrow 0$ whereas $E_{\mathrm{SLG}, 0}^{(\tau, n>1)} \rightarrow \infty$, as was seen in Sec. 3.1.1. These limits are easily detected in the conductance since a distinct resonance centers around $\phi=(m-1 / 2) \phi_{0}$ for $\delta \theta_{\mathrm{g}}^{(1)} \rightarrow 0$ and around $\phi=m \phi_{0}$ for $\delta \theta_{\mathrm{g}}^{(n>1)} \rightarrow-1$, as described in Sec. (5.1.1).

The physical interpretation of this behaviour is that the lowest-subband exhibits pure "Dirac" features as we move deeper into the inverted-gap regime, i.e. the topological regime. This is due to $\theta_{\mathrm{g}}^{(\tau, 1)} \rightarrow 2 \pi \phi / \phi_{0}+\pi$ and thus retaining the spin- $1 / 2 \pi$ Berry phase factor that arises from the Dirac nature of the material.

Higher subbands, on the other hand, behave non-relativistically, i.e. in 
a "Schrödinger"-like fashion, in this limit because they effectively possess $\theta_{\mathrm{g}}^{(\tau, n>1)} \rightarrow 2 \pi \phi / \phi_{0}$ (n.b. a shift of $2 m \pi$ can be neglected). This shows that, unless a subband is topologically protected, increasing the magnitude of the gap size causes the system to become more Schrödinger-like.

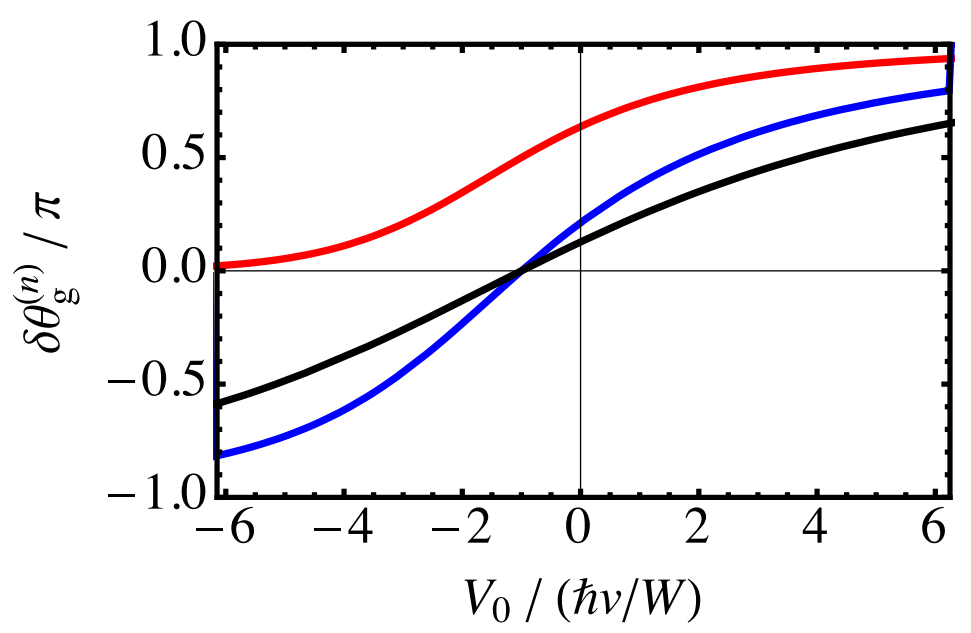

Figure 6.1: Figure of $S L G$ Berry phase $\delta \theta_{\mathrm{g}}^{(n)}$ as a function of the universal parameter $V_{0} W / \hbar v$ for the three lowest-subband. The different subbands are given by $n=1$ (red), 2 (blue) and 3 (black). The behaviour of the lowest subband can be seen to be distinctly different from the higher subbands.

Furthermore, for the $n>1$ subbands we also notice that $\delta \theta_{\mathrm{g}}^{(n>1)}=0$ at $V_{0} W / \hbar v=-1$. Here we remind the reader that $V_{0} W / \hbar v=-1$ is also the value at which the system transitions between being topologically trivial or non-trivial, as shown in Eqn. (3.13). We see that this is also the point at which $\delta \theta_{\mathrm{g}}^{(n>1)}$ transitions from negative to positive values (as $V_{0} W / \hbar v$ is increased). Naturally this outcome parallels those of the $\tau$-valley dispersion shifts results that were described in Sec. 3.3.1. With regards to the $n>$ 1 subband conductance, this transition point should be detectable since it involves the swapping of the $\tau$-valley conductance peaks around $\phi=(m-$ $1 / 2) \phi_{0}$ [as was seen for SQW in Fig. (5.8b)].

The situation for the lowest subband is entirely different since $\delta \theta_{\mathrm{g}}^{(1)}$ stays positive for the entire domain. At $V_{0} W / \hbar v=-1, \delta \theta_{\mathrm{g}}^{(1)}=\pi / 2$ and thus is the only subband that does not have a zero $\delta \theta_{\mathrm{g}}^{(n)}$ value. This particular system parameter value is also significant for the $\phi$ dependence of the conductance spectrum since it is the transition point for the centering of the conductance resonance areas around either $\phi=(m-1 / 2) \phi_{0}$ or $\phi=m \phi_{0}$, as given by 
Eqn. (5.15). At this point, the conductance at $\phi=(m-1 / 2) \phi_{0}$ and $\phi=m \phi_{0}$ will be of equal amplitude because the contributions from the $\pm \tau$ peaks are equally distant from both points (due to being separated by $\phi=\phi_{0} / 2$ ). This results in $\phi$ having a periodicity of $\phi_{0} / 2$ in the lowest subband conductance, as discussed in Sec. (5.1.1).

Thus, $V_{0} W / \hbar v=-1$ separates $\delta \theta_{\mathrm{g}}^{(n)}$ into two distinct regions that correspond to the non-topological and topological regimes, respectively. This result is in complete agreement to the previous conclusions in the Chapter 3 . In addition to this, we have also found that the limit of $\delta \theta_{\mathrm{g}}^{(n)}$ in the deeply inverted regime differ between the topological subband $(n=1)$ and the bulk subbands $(n>1)$. We can conclude that, in addition to results in Sec. (3.1.1), there are numerous signatures of the topological phase in SLG QRs when $V_{0} W / \hbar v \leq-1$.

We have now completed our analysis of the SLG QR Berry phase. As we proceed to the energy-reflection symmetric case of SQW rings, we hope to see some of these features paralleled.

\subsection{Semiconductor quantum well}

\subsubsection{Energy-reflection symmetric case}

To gain an in-depth understanding of the general SQW QR Berry phase, we first consider the simplified energy-reflection symmetric SQW model with $\tilde{D}=0$. It turns out that one can simplify the subband energy dispersion [Eqn. 4.49] ] such that one can obtain an approximate, but accurate, $\delta \theta_{\mathrm{g}}^{(n)}$ equation:

$$
\begin{aligned}
\delta \theta_{\mathrm{g}}^{(n)} & =\frac{2 \Lambda_{\mathrm{SQW}} \tilde{E}_{\mathrm{in}}}{\Delta_{\mathrm{SQW}}^{2}-\left(\frac{W}{R}\right)^{2} \Lambda_{\mathrm{SQW}}^{2}+2 \tilde{E}_{\mathrm{in}} \Upsilon_{\mathrm{SQW}}} \\
& \approx \frac{2 \Lambda_{\mathrm{SQW}} \tilde{E}_{\mathrm{in}}}{\Delta_{\mathrm{SQW}}^{2}+2 \tilde{E}_{\mathrm{in}} \Upsilon_{\mathrm{SQW}}}
\end{aligned}
$$

where $E_{\mathrm{SQW}, 0}^{(\tau, n)}=-E_{\mathrm{SQW}, 0}^{(\tau,-n)}, \Lambda_{\mathrm{SQW}}=\Lambda_{\mathrm{SQW}}^{\prime}$ and $\Upsilon_{\mathrm{SQW}}=-\Upsilon_{\mathrm{SQW}}^{\prime}$. Notice that, once again, $\delta \theta_{\mathrm{g}}^{(n)}=-\delta \theta_{\mathrm{g}}^{(-n)}$. To obtain this result we also discard the $\epsilon^{3}$ and 
$\epsilon^{4}$ terms that appear in the energy dispersion when brackets are expanded. Naturally, since we are interested in energies that are close to $E_{\mathrm{SQW}, 0}^{(\tau, n)}$, we can simplify this to

$$
\delta \theta_{\mathrm{g}}^{(n)} \approx \frac{2 \Lambda_{\mathrm{SQW}} \tilde{E}_{\mathrm{SQW}, 0}^{(\tau, n)}}{\Delta_{\mathrm{SQW}}^{2}+2 \tilde{E}_{\mathrm{SQW}, 0}^{(\tau, n)} \Upsilon_{\mathrm{SQW}}}
$$

The form of $\delta \theta_{\mathrm{g}}^{(n)}$ for the $n=1,3$ subbands of a SQW ring at a fixed, large $q_{0} W$ value can be seen in Fig. 6.2). In this figure, we also show the differences due to the inclusion of the $l^{2}$ term in $H_{\mathrm{SQW}, 1}^{(\tau)}$ (i.e. the effects of the $\Upsilon_{\mathrm{SQW}}$ element) and, for comparison, the SLG $\delta \theta_{\mathrm{g}}^{(n)}$ value.

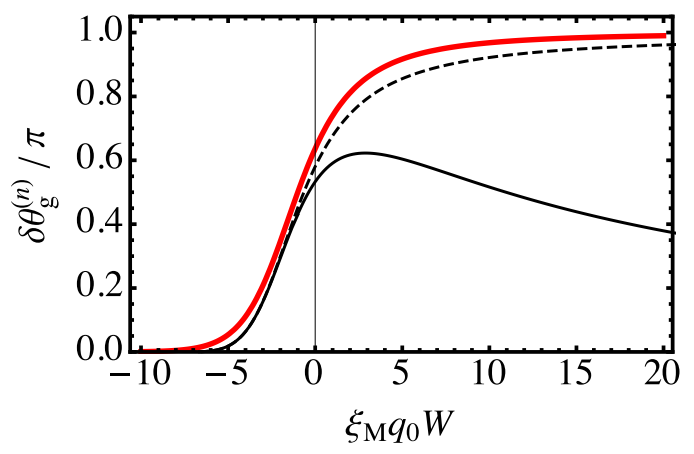

(a) $n=1$ subband

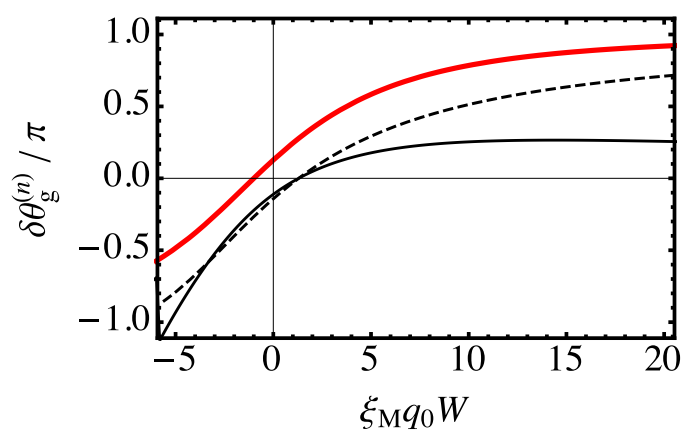

(b) $n=3$ subband

Figure 6.2: Figure of $n=1$ (a) and 3 (b) subband $\delta \theta_{\mathrm{g}}^{(n)}$ as a function of $\xi_{\mathrm{M}} q_{0} W \equiv V_{0} /(\hbar v / W)$ for $S L G$ (red) and $S Q W$ (black). For the $S Q W \delta \theta_{\mathrm{g}}^{(n)}$ values, we use Eqn. (5.31a) (dashed-black) and the more accurate solution (when $H_{\mathrm{SQW}, 1}^{(\tau)}$ includes the $l^{2} / r^{2}$ term) as given by Eqn. 6.4) (solid-black). (a) We see that leaving out the $l^{2} / r^{2}$ term causes the $S Q W$ phase to be very similar to that of SLG - the difference is solely due to the additional wing states in $S Q W$. The inclusion of the $l^{2} / r^{2}$ causes a suppression of the Berry phase for large $\xi_{\mathrm{M}} q_{0} W$. (b) Even without the $l^{2} / r^{2}$ term, there are discrepancies between $S L G$ and $S Q W \delta \theta_{\mathrm{g}}^{(n)}$ values. This seems to be caused due to a shift in the overall subband energy dispersions. Other parameters were held constant at $q_{0} W=26.6, W / R=0.1$ and $\tilde{D}=0$.

For $n=1$ the exclusion of the $l^{2}$ terms leads to a Berry phase that strongly resembles that of SLG as can be seen in Fig. 6.2a). The differences can be accounted due to the appearance of additional wing states in the SQW model [see Eqns. (4.35) and (4.38)] which have an overall small effect on the phase. Once we include the $l^{2}$ term, $\delta \theta_{\mathrm{g}}^{(n)}$ becomes suppressed for large values of $\xi_{\mathrm{M}} q_{0} W$ which causes the black curve to tend to $\delta \theta_{\mathrm{g}}^{(n)}=0$ in the 
$\xi_{\mathrm{M}} \rightarrow \infty$ limit as compared to $\delta \theta_{\mathrm{g}}^{(n)}=\pi$ for the other curves. The reason underlying this feature is that as $\xi_{\mathrm{M}} \rightarrow \infty$, the magnitude of the $E_{\mathrm{SQW}, 0}^{(\tau, n)} \Upsilon_{\mathrm{SQW}}$ term increases linearly, which can be seen in Fig. (6.3b), and since this term appears in the denominator of the phase [see Eqn. [6.4] ], it causes an overall decrease in $\delta \theta_{\mathrm{g}}^{(n)}$. The linear increase is because $E_{\mathrm{SQW}, 0}^{(\tau, n)} \propto \xi_{\mathrm{M}}$ [see Fig. 4.1)] and $\Upsilon_{\mathrm{SQW}}$ is a constant [see Fig. (6.3a)] at large $\xi_{\mathrm{M}}$ value.

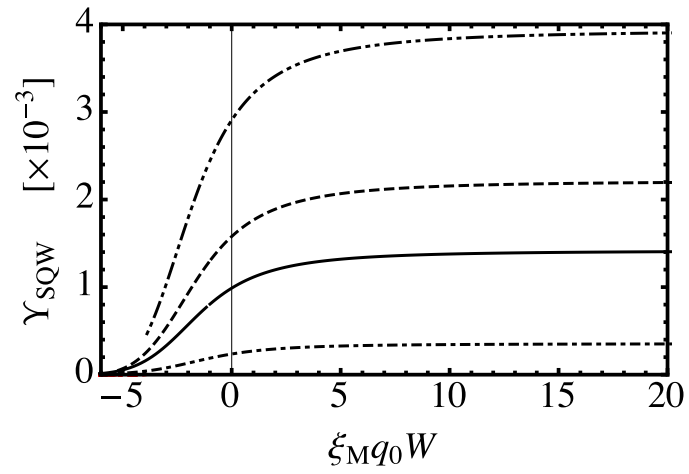

(a) $\Upsilon_{\mathrm{SQW}}$ versus $\xi_{\mathrm{M}}$

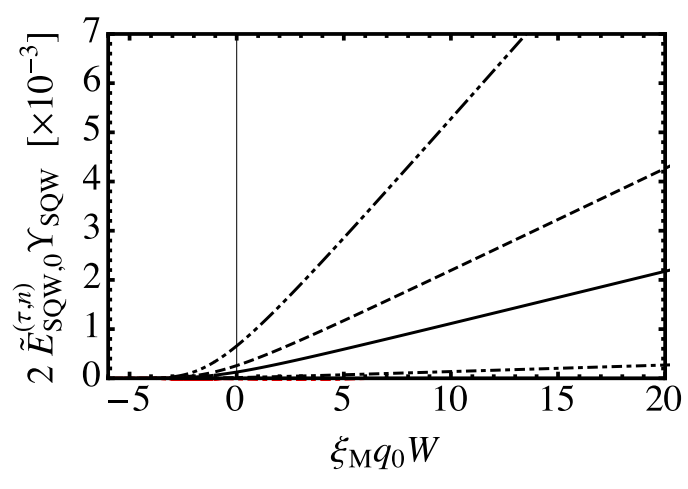

(b) $2 E_{\mathrm{SQW}, 0}^{(\tau, n)} \Upsilon_{\mathrm{SQW}}$ versus $\xi_{\mathrm{M}}$

Figure 6.3: Figure of $\Upsilon_{\mathrm{SQW}}(\mathbf{a})$ and $2 E_{\mathrm{SQW}, 0}^{(\tau, n)} \Upsilon_{\mathrm{SQW}}$ (b) as a function of $\xi_{\mathrm{M}} q_{0} W$ for a $S Q W Q R$ at different widths. The different widths are given by $q_{0} W=15.9$ (dot-dot-dashed), 21.3 (dashed), 26.6 (solid) and 53.1 (dotdashed). (a) We see that, as the width is decreased, the overall $\Upsilon_{\mathrm{SQW}}$ magnitude increases. Additionally, $\Upsilon_{\mathrm{SQW}}$ begins to saturate at a fairly small gap size for all widths. (b) Since the energy increases linearly as a function of $\xi_{\mathrm{M}}\left(\right.$ for large $\left.\xi_{\mathrm{M}}\right)$, the $2 E_{\mathrm{SQW}, 0}^{(\tau, n)} \Upsilon_{\mathrm{SQW}}$ term, which appears in $\delta \theta_{\mathrm{g}}^{(n)}$, also becomes linearly dependent on $\xi_{\mathrm{M}}$. The other parameters were held constant at $W / R=0.1$ and $\tilde{D}=0$.

Physically, the $l^{2}$ term can be interpreted as a radially-dependent confinement that is proportional to $1 / r^{2}$. Thus at certain parameter values, it may confine the wave to an outer section of the ring since the potential at the inner part is very high. As we increase the gap size, this causes the confinement to become stronger and eventually pushes the effective width, within which the wave is situated, to zero causing $\delta \theta_{\mathrm{g}}^{(n)} \rightarrow 0$.

For $n=3$, the differences between SLG and SQW become more apparent even when $l^{2} / r^{2}$ is excluded. The SQW curves seem to be shifted to the right of SLG which could correspond to a shift of the $\tau=1$ energy dispersion relation to the right due to the additional wing states. However another possible explanation for this effect may be due to the fact that we ignore the 
inter-subband interactions. Higher subbands may unevenly repel the $n=3$ dispersion curve causing a shift in the Berry phase.

For $n=3$, when the effects of the $\Upsilon_{\mathrm{SQW}}$ terms (i.e. the $l^{2}$ terms in the Hamiltonian) are included, we see that for $\xi_{\mathrm{M}}>0$ the Berry phase decreases in the same manner as for $n=1$. However, for $\xi_{\mathrm{M}}<0, \delta \theta_{\mathrm{g}}^{(n)}$ becomes larger in magnitude as compared to the $\delta \theta_{\mathrm{g}}^{(n)}$ described by Eqn. 5.31a, which indicates that at some point around $\xi_{\mathrm{M}} q_{0} W \approx-3$, the $\Upsilon_{\mathrm{SQW}}$ term becomes negative thus decreasing the denominator value. The actual value of $\Upsilon_{\mathrm{SQW}}$ can be seen in Fig. (6.4), which corroborates this theory, since at $\xi_{\mathrm{M}} q_{0} W \approx$ -3 , the $\Upsilon_{\mathrm{SQW}}$ term becomes negative. As it becomes larger in magnitude (while still negative), $\delta \theta_{\mathrm{g}}^{(n)}$ approaches infinity since $\Upsilon_{\mathrm{SQW}} \rightarrow-\left|\Delta_{\mathrm{SQW}}\right|^{2}$. Similar results can be anticipated for all $n>1$ subbands [e.g. see $n=2$ in Figs. 6.4 and 6.6)].

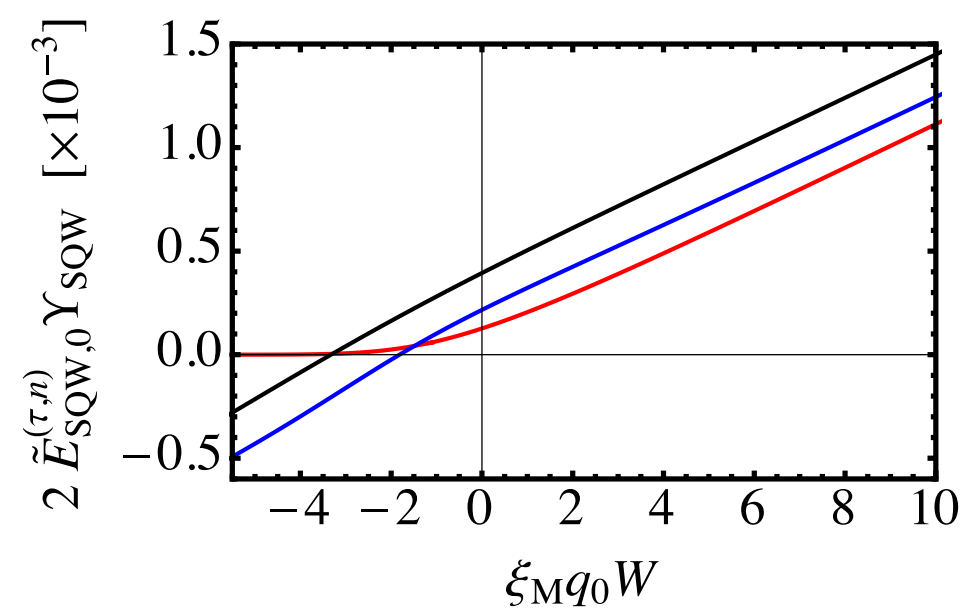

Figure 6.4: Figure of $2 \tilde{E}_{\mathrm{SQW}, 0}^{(\tau, n)} \Upsilon_{\mathrm{SQW}}$ as a function of $\xi_{\mathrm{M}} q_{0} W \equiv V_{0} /(\hbar v / W)$ for subbands $n=1$ (red), 2 (blue) and 3 (black). The lowest subband is the only subband that does not become negative eventually. Other parameters were held constant at $q_{0} W=26.6, W / R=0.1$ and $\tilde{D}=0$.

In Fig. 6.5 we plot the dependence of $\delta \theta_{\mathrm{g}}^{(n)}$ as a function of $\xi_{\mathrm{M}} q_{0} W$ for different $q_{0} W$ values. We see that $\delta \theta_{\mathrm{g}}^{(n)}$, unlike the SLG QR, does not form a universal curve since the curve drastically changes when the width is altered. This is due to the non-universality of the secular energy equation in SQWs. Note that some of the curves do not stretch over the complete region since we only consider $\xi_{\mathrm{M}}>-\frac{1}{4}$ [recall from Eqn. (4.10] ] which cuts off data for some of the curves that correspond to smaller values of $q_{0} W$. 


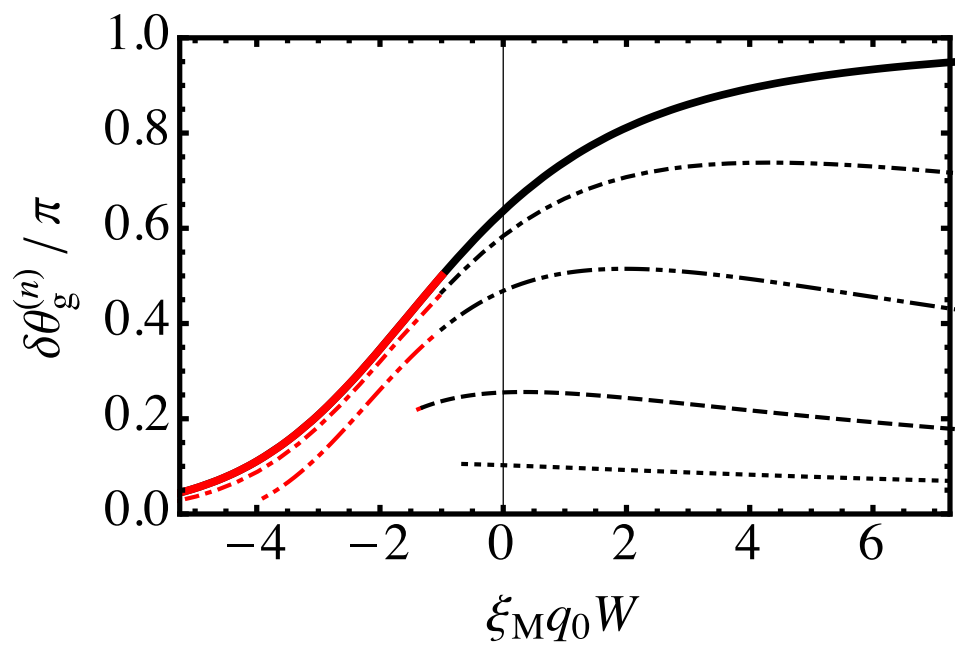

Figure 6.5: Figure of $\delta \theta_{\mathrm{g}}^{(n)}$ as a function of $\xi_{\mathrm{M}} q_{0} W \equiv V_{0} /(\hbar v / W)$ for $S L G$ (solid) and $S Q W$ (discontinuous) QRs for different widths. The different widths are given by $q_{0} W=2.66$ (dotted), 5.66 (dashed), 15.9 (dot-dot-dashed) and 53.1 (dot-dashed). The transition from two evanescent states $\left(\left|E_{\mathrm{SLG}, 0}^{(\tau, n)}\right|<\right.$ $\left.\left|\xi_{\mathrm{M}}\right|\right)$ to zero evanescent states $\left(\left|E_{\mathrm{SLG}, 0}^{(\tau, n)}\right| \geq\left|\xi_{\mathrm{M}}\right|\right)$ are represented by red and black lines, respectively. We see that as the width is decreased, the overall magnitude of the phase decreases significantly due to the increase of $\Upsilon_{\mathrm{SQW}}$. Note that some of the curves do not stretch over the complete region since we only consider $\xi_{\mathrm{M}}>-\frac{1}{4}$. The parameters were held constant at $W / R=0.1$ and $\tilde{D}=0$. 
For small $q_{0} W$, the Berry phase appears to shrink in magnitude. This is because as the radius and width are both decreased, the $l^{2} / r^{2}$ confinement of the particle becomes steeper and larger which causes it to more effectively confine higher energy particles in an effective width that is smaller than the actual width of the ring. Thus the $\Upsilon_{\mathrm{SQW}}$ term becomes larger for a smaller width [see Fig. 6.3a)] and causes the suppression of $\delta \theta_{\mathrm{g}}^{(n)}$ phase. As the width is increased, the $l^{2} / r^{2}$ potential confines the wave less and less thus increasing the overall magnitude of $\delta \theta_{\mathrm{g}}^{(n)}$. However, we still find that at a large enough value of $\xi_{\mathrm{M}}$, the $l^{2} / r^{2}$ eventually becomes the dominant effect which causes $\delta \theta_{\mathrm{g}}^{(n)}$ to approach 0 .

Fig. (6.5) also shows the transition between two to zero evanescent states (red to black). These transitions seem to happen at around $\xi_{\mathrm{M}} q_{0} W \approx-1$ for large widths, which agrees with the predictions of Eqn. 4.28.

As a direct comparison to Fig. 6.1), we plot Fig. 6.6 for which $q_{0} W=$ 26.61 . The forms of the SQW curves are similar to SLG since the $n>1$ subbands transition between positive and negative values of $\delta \theta_{\mathrm{g}}^{(n)}$ while the $n=1$ subband stays purely positive. Once again, this demonstrates the distinct difference between the lowest and higher subbands, that is indicative of the lowest subband's unigue topological properties. The major visible differences between the SQW and SLG QR $\delta \theta_{\mathrm{g}}^{(n)}$ are due to the $\Upsilon_{\mathrm{SQW}}$ term which causes $\delta \theta_{\mathrm{g}}^{(n)} \rightarrow 0$ as $\xi_{\mathrm{M}} \rightarrow \infty$ and $\delta \theta_{\mathrm{g}}^{(n)} \rightarrow-\infty$ as $\xi_{\mathrm{M}} \rightarrow-\left|\Delta_{\mathrm{SQW}}\right|^{2}$.

In Fig. 6.6), the $\delta \theta_{\mathrm{g}}^{(n>1)}=0$ points are naturally the same as those in Fig. (4.8) since they are both proportional to $\Lambda_{\mathrm{SQW}}$. However, unlike SLG, there is no clear indication in $\delta \theta_{\mathrm{g}}^{(n)}$ of the value at which SQW becomes topological since $\delta \theta_{\mathrm{g}}^{(n>1)}=0$ does not seem to occur at $\xi_{\mathrm{Mc}} q_{0} W$ [see Eqn. 4.28]. Thus, we only know that the system is in the topological regime when the eigenstate becomes purely evanescent which occurs at $\xi_{\mathrm{M}} q_{0} W \approx-1$, as derived in Sec. 4.1.2).

Unfortunately, since the $2 E_{\mathrm{SQW}, 0}^{(\tau, n)} \Upsilon_{\mathrm{SQW}}$ generally suppresses the magnitude of $\delta \theta_{\mathrm{g}}^{(n)}$, we also do not possess the same distinct conductance patterns as described in the SLG QR section. For example, when $\xi_{\mathrm{M}} q_{0} W=\xi_{\mathrm{Mc}} q_{0} W$, the Berry phase of the lowest subband is not equal to $\pi / 2$ which means

\footnotetext{
${ }^{1}$ Naturally, we have already discovered that $\delta \theta_{\mathrm{g}}^{(n)}$ will change with respect to $q_{0} W$ and hence this is not quite a "direct" comparison. However, we choose a large value of $q_{0} W$ which should be most similar to the SLG case.
} 


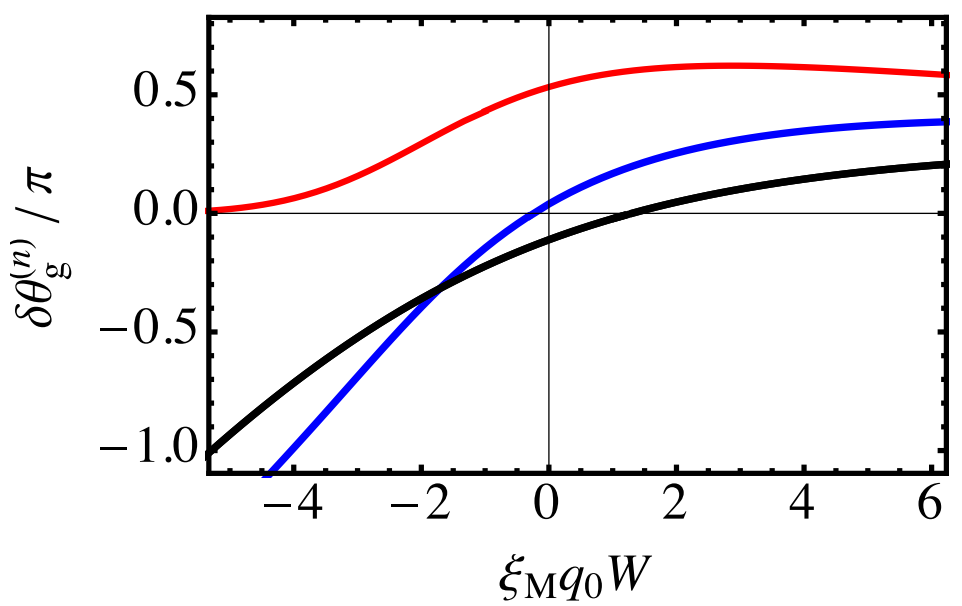

Figure 6.6: Figure of the $S Q W Q R \delta \theta_{\mathrm{g}}^{(n)}$ as a function of $\xi_{\mathrm{M}} q_{0} W$ for different subbands. We used the general numerical solution derived from Eqn. (6.4). The different subbands are given by $n=1$ (red), 2 (blue) and 3 (black). The parameters were held constant at $W / R=0.1, \tilde{D}=0$ and $q_{0} W=26.6$.

that the conductance resonance regions do not swap their centering between $\phi=(m-1 / 2) \phi$ and $\phi=m \phi$.

Now that we have analysed the differences between the SLG and energyreflection symmetric SQW QR cases, we can move onto studying the most complicated system: non-energy-reflection symmetric SQW QRs.

\subsubsection{Non-energy-reflection symmetric case}

Applying the same procedure as for the $\tilde{D}=0$ case, we can obtain an accurate expression for the Berry phase with a general $\tilde{D}$ value, by implementing the same realistic approximations. Doing this gives

$$
\delta \theta_{\mathrm{g}}^{(n)} \approx \frac{\left(\Lambda_{\mathrm{SQW}}+\Lambda_{\mathrm{SQW}}^{\prime}\right) \tilde{E}_{\mathrm{in}}-\Lambda_{\mathrm{SQW}} \tilde{E}_{\mathrm{SQW}, 0}^{(\tau,-n)}-\Lambda_{\mathrm{SQW}}^{\prime} \tilde{E}_{\mathrm{SQW}, 0}^{(\tau, n)}}{\Delta_{\mathrm{SQW}}^{2}+\left(\tilde{E}_{\mathrm{in}}+\frac{\tilde{E}_{\mathrm{SQW}, 0}^{(\tau, n)}}{2}-\frac{3}{2} \tilde{E}_{\mathrm{SQW}, 0}^{(\tau,-n)}\right) \frac{\Upsilon_{\mathrm{SQW}}}{2}+\left(\tilde{E}_{\mathrm{in}}+\frac{E_{\mathrm{SQW}, 0}^{(\tau,-n)}}{2}-\frac{3}{2} \tilde{E}_{\mathrm{SQW}, 0}^{(\tau, n)}\right) \frac{\Upsilon_{\mathrm{SQW}}^{\prime}}{2}}
$$


which simplifies to

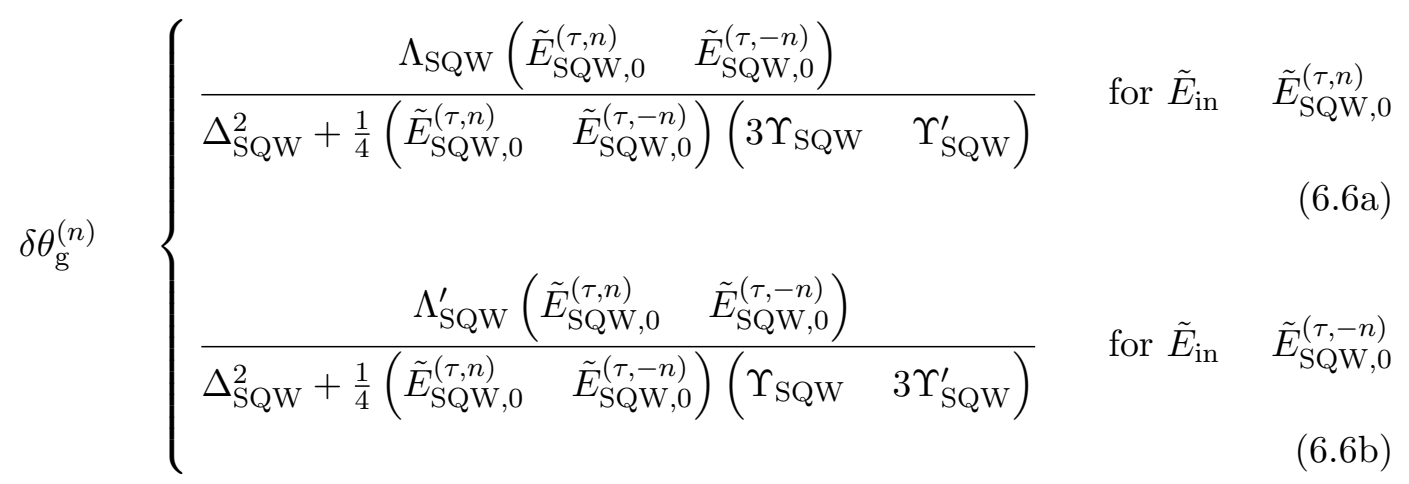

where we have used a similar line of reasoning as for the $\tilde{D}=0$ case. One notices that $\delta \theta_{\mathrm{g}}^{(n)} \sigma-\delta \theta_{\mathrm{g}}^{(-n)}$ since energy-reflection symmetry does not hold any more. The $\tilde{D}$ terms are associated with a $\sigma_{0}$ term which means it acts differently on electrons and holes since it effectively couples to the charge of the particle. This causes the asymmetry in the energies of the conduction $(n>0)$ and valence $(n<0)$ subbands. For the simplicity in the next sections we define

$$
\Delta \Upsilon_{\mathrm{SQW}} \equiv \begin{cases}3 \Upsilon_{\mathrm{SQW}}-\Upsilon_{\mathrm{SQW}}^{\prime} & \text { for } \tilde{E}_{\mathrm{in}} \approx \tilde{E}_{\mathrm{SQW}, 0}^{(\tau, n)} \\ \Upsilon_{\mathrm{SQW}}-3 \Upsilon_{\mathrm{SQW}}^{\prime} & \text { for } \tilde{E}_{\mathrm{in}} \approx \tilde{E}_{\mathrm{SQW}, 0}^{(\tau,-n)}\end{cases}
$$

and $\Delta \tilde{E}_{\mathrm{SQW}}^{(\tau, n)} \equiv \tilde{E}_{\mathrm{SQW}, 0}^{(\tau, n)}-\tilde{E}_{\mathrm{SQW}, 0}^{(\tau,-n)}$. Since the asymmetry in the system is already present in the size-quantisation energies, $\tilde{E}_{\mathrm{SQW}, 0}^{(\tau, \pm n)}$, the $\tilde{D} l^{2} / \tilde{r}^{2} \sigma_{0}$ only adds further complications due to its additional asymmetry.

In Fig. 6.7), we show the lowest-subband $\delta \theta_{\mathrm{g}}^{(n)}$ as a function of $\xi_{\mathrm{M}} q_{0} W$ for different values of $\tilde{D}$. We see that the peak of the Berry phase moves from the left to the right as we increase $\tilde{D}$ from negative to positive. This is because the $\Delta \Upsilon_{\mathrm{SQW}}$ (in this case $=3 \Upsilon_{\mathrm{SQW}}-\Upsilon_{\mathrm{SQW}}^{\prime}$ ) term saturates to a smaller magnitude for an increasing $\tilde{D}$ as demonstrated in Fig. 6.8a). This causes the denominator to become smaller thus allowing for the peak to shift to the right. We can also see a larger negative $\tilde{D}$ curve approaches 0 quicker than the less negative curves due to the same reason: larger saturation magnitude of $\Upsilon_{\mathrm{SQW}}$ for the more negative curves thus causing a quicker decrease. Additionally, we can see regions of $\xi_{\mathrm{M}} q_{0} W$ for which there are 0 (black), 1 (blue) or 2 (red) evanescent topological bound states within the band gap $\pm\left|\xi_{\mathrm{M}}\right|$. 


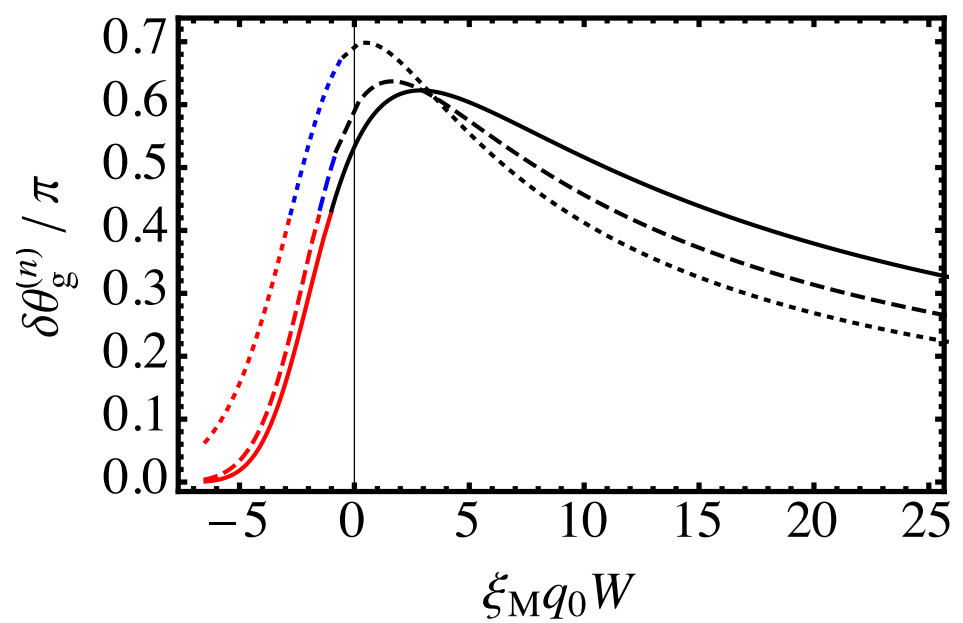

Figure 6.7: Figure of the lowest subband $n=1 \delta \theta_{\mathrm{g}}^{(n)}$ as a function of $\xi_{\mathrm{M}} q_{0} W$ for a $S Q W Q R$ at differing values of $\tilde{D}$. For the $S Q W$ curves, we used the general solution, given Eqn. (6.6a). The different $\tilde{D}$ values are given by $D /|B|=-0.746$ (dotted), -0.373 (dashed) and 0 (solid). Here we have also depicted the presence of 2 (red), 1 (blue) or 0 (black) evanescent states within $\left|\xi_{\mathrm{M}}\right|$. The parameters were held constant at $W / R=0.1$ and $q_{0} W=26.6$.

These points are theoretically predicted to occur in Sec. 4.1.2).

In Fig. 6.8, we see that $\Delta \Upsilon_{\mathrm{SQW}}$ and $\Delta \tilde{E}_{\mathrm{SQW}, 0}^{(\tau, n)} \Delta \Upsilon_{\mathrm{SQW}}$ behave oppositely for $\pm n$. In the case of $n=1$, as shown in Fig. (6.7), we saw that the saturation point of $\Delta \Upsilon_{\mathrm{SQW}}$ was higher than when $\tilde{D}=0$ which caused a peak shift to the left. For $n=-1$, we would expect the opposite since the saturation point of $\Delta \Upsilon_{\mathrm{SQW}}$ is lower than that of $\tilde{D}=0$ which would mean that the peak shifts towards the right 2 ,

We compare the magnitudes of $\Delta \tilde{E}_{\mathrm{SQW}, 0}^{(\tau, n)} \Delta \Upsilon_{\mathrm{SQW}}$ as a function of gap size for different subbands in Fig. 6.9. Here, once again, the $\tilde{D}=0$ curves [seen in Fig. (6.4)] split into two curves that correspond to the $\pm n$ subbands. Furthermore, we see that the $|n|>1$ all cross between negative and positive values. When they are negative, the $\delta \theta_{\mathrm{g}}^{(|n|>1)}$ curves can tend to $\pm \infty$ when $\Delta \tilde{E}_{\mathrm{SQW}, 0}^{(\tau, n)} \Delta \Upsilon_{\mathrm{SQW}} \rightarrow-4\left|\Delta_{\mathrm{SQW}}\right|^{2}$.

In Fig. 6.10 we have plotted $\delta \theta_{\mathrm{g}}^{(n)}$ as a function of $\xi_{\mathrm{M}}$ for the $n= \pm 1, \pm 2, \pm 3$ subbands. We once again see that the Berry phase shows characteristic differences between the lowest $|n|=1$ subbands and the higher $|n|>1$ subbands. The $|n|=1$ subbands, which becomes topological for the deeply

\footnotetext{
${ }^{2}$ This expected shift can be seen in Fig. 6.10
} 


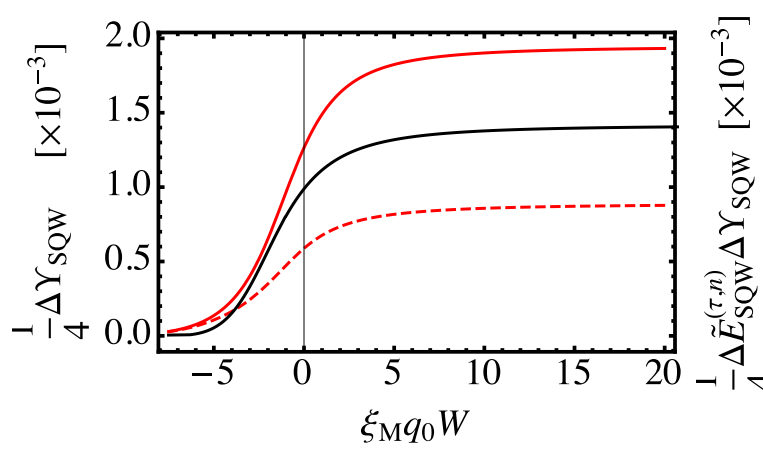

(a) $n=1$ subband

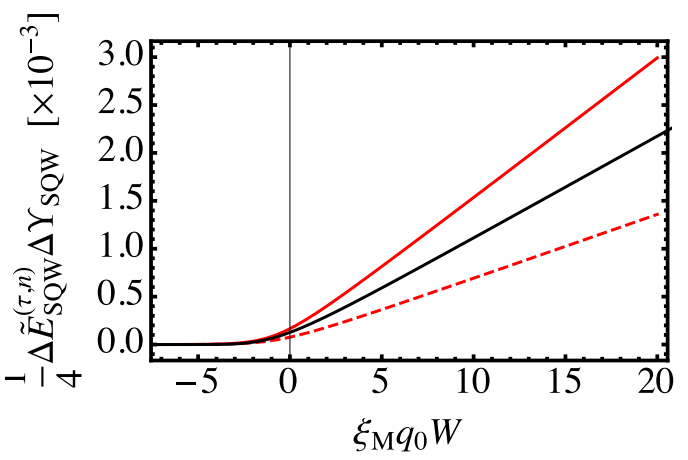

(b) $n=3$ subband

Figure 6.8: Figure of $S Q W Q R \Delta \Upsilon_{\mathrm{SQW}} \equiv 3 \Upsilon_{\mathrm{SQW}}-\Upsilon_{\mathrm{SQW}}^{\prime}$ (solid) or $\Upsilon_{\mathrm{SQW}}-$ $3 \Upsilon_{\mathrm{SQW}}^{\prime}$ (dashed) (a) and $\frac{1}{4} \Delta \tilde{E}_{\mathrm{SQW}}^{(\tau, n)} \Delta \Upsilon_{\mathrm{SQW}}$ (b) as a function of $\xi_{\mathrm{M}} q_{0} W$ for $\tilde{D}=0$ (black) and -0.746 (red) at a constant width $q_{0} W=26.6$. In both figures we can see that turning on the $\tilde{D}$ factor causes the $\tilde{D}=0 \Upsilon_{\mathrm{SQW}}$ term in Eqn. (6.4) to split into the two $\Delta \Upsilon_{\mathrm{SQW}}$ terms. The direction of splitting depend on the type of subband: solid red for type (a) and dashed red for type (b) states. Other parameters were held constant at $W / R=0.1$ and $\tilde{D}=-0.746$.

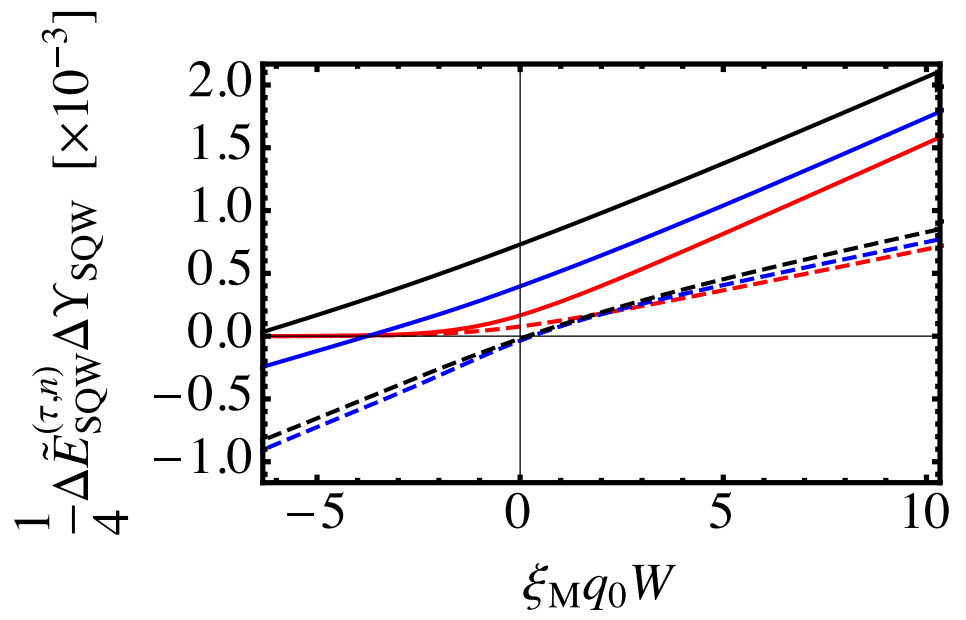

Figure 6.9: Figure of $\frac{1}{4} \Delta \tilde{E}_{\mathrm{SQW}}^{(\tau, n)} \Delta \Upsilon_{\mathrm{SQW}}$ as a function of $\xi_{\mathrm{M}} q_{0} W$ for different $S Q W$ QR subbands $n= \pm 1$ (solid/dashed red), \pm 2 (solid/dashed blue) and \pm 3 (solid/dashed black). Other parameters were held constant at $W / R=0.1$, $q_{0} W=26.6$ and $\tilde{D}=-0.746$. 
inverted regime, do not possess zero crossing points where $\delta \theta_{\mathrm{g}}^{(n)}=0$, as was indicated by the lack of $\tau$ flavour dispersion swapping in Sec. (4.2.2). This is also supported by the results in the SQW conductance [as seen in Fig. (5.8a)] where we saw no $\tau$ flavour swapping of the conductance peaks.

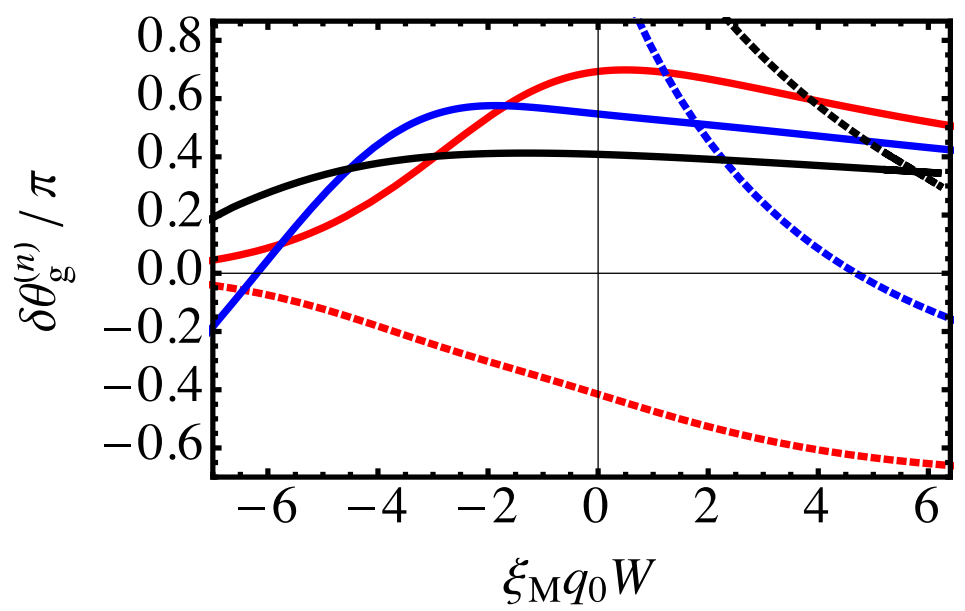

Figure 6.10: Figure of $\delta \theta_{\mathrm{g}}^{(n)}$ as a function of $\xi_{\mathrm{M}} q_{0} W$ for a $S Q W Q R$ for different subbands: $n= \pm 1$ (solid/dotted red), \pm 2 (solid/dotted blue) and $\mathbf{\pm}$ (solid/dotted black). We used the general solution derived from Eqns. (6.6a) and $(6.6 b)$. The parameters were held constant at $\tilde{D}=-0.746, W / R=0.1$ and $q_{0} W=26.6$.

However for $|n|>1$, we do observe these zero crossing points, i.e. $\delta \theta_{\mathrm{g}}^{(n>1)}=0$, which occur at the same gap value as was predicted by plotting $\Lambda_{\mathrm{SQW}}$ and $\Lambda_{\mathrm{SQW}}^{\prime}$ in Fig. (4.11). This was also seen in the conductance where the $\tau$ flavour conductance peaks swapped positions [see Fig. (5.8b)]. Unfortunately, like the $\tilde{D}=0$ case, these zero points do not occur at the critical gap values, $\xi_{\mathrm{Mc} \pm}$, that separate the topological and trivial regimes, as was discussed in Sec. 4.1.2). Thus, the only measure of the gap value at which the transition occurs, is found using the theoretical predictions in Sec. 4.1.2). However, we should still be able to measure the topological properties of the lowest subband by observing the unique Berry phase of this state.

Another feature that we notice in the $|n|=1$ subbands is that, unlike the $|n|>1$ subbands, the curves do not appear to shift significantly and thus still strongly resemble those of the $\tilde{D}=0$ SQW and SLG cases. The higher subbands are significantly shifted due to the distorted $\Upsilon_{\mathrm{SQW}}$ values. It may also be possibly due to the inaccuracy of the higher subband dispersion equations, arising from the perturbation theory approximations. 
We have now explored and understood many aspects of $\delta \theta_{\mathrm{g}}^{(n)}$ for SLG, TMD and SQW QRs. Although there are still areas that are worth investigating such as the exact effects of $\Delta_{\mathrm{SQW}}$ in the SQW systems, we leave this for another project. In the next chapter, we summarise our results, discuss some of the implication of these observations and conclude with an outlook for future work. 


\section{Chapter 7}

\section{Summary and outlook}

In this thesis we have undertaken a comprehensive analytical study of the confinement effects and transport properties in single-layer graphene (SLG), transition-metal dichalcogenide (TMD) and semiconductor quantum well (SQW) quantum rings (QRs). We began by developing a general methodology that was modified to tackle each material individually. In this chapter we will summarise the main results, their implications, and provide possibilities for further work.

For SLG QRs, we obtained a general formula [given in Eqn. (3.38)] that describes the subband dispersion of the system as a function of the confinement energy, $\hbar v / W$, and gap size, $V_{0}$, for a small aspect ratio, $\epsilon \equiv W / R \quad 1$. Unlike previous works for $V_{0}=0$ 66 68, we have accounted for the sizequantisation energy, $E_{\mathrm{SLG}, 0}^{(\tau, n)}$, which introduces a finite mass gap. We have also included the diagonal $2 \times 2$ block Hamiltonian $\mathrm{H}_{\mathrm{SLG}, 1}^{(\tau, n)}$ term [see Eqn. 3.22] that cause time-reversal flavour dispersion shifts. These terms turn out to be vital for obtaining an accurate Berry phase. The only exception to this general lack of terms is the work done by Recher et. al. on the $V_{0}=0$ SLG QR system [5]. Literature regarding SLG systems with a general $V_{0}$ also do not include the diagonal $\mathrm{H}_{\mathrm{SLG}, 1}^{(\tau, n)}$ term, $\Gamma_{\mathrm{SLG}}$ [Eqn. 3.37)], which they may have assumed to be negligible since it is "just" an (angular momentum dependent) energy shift. However, we have shown that this term has a substantial effect on the dispersions, conductances and the Berry phase and hence must be taken into account in these contexts.

Results for TMD QRs are qualitatively similar to those of the SLG QR with 
however the noticeable addition of the large valence band spin-splitting. The general subband dispersion was found in Sec. (3.3.2) which is in agreement with the work of Oliveira et.al. [8]. However, they also neglected the diagonal $\Gamma_{\mathrm{TMD}}$ term in their Hamiltonian which, as in the case of SLG, we have found necessary to include for a complete understanding of the Berry phase and conductance [see Sec. (5.3)].

General SQW systems have been analytically explored in various studies 7 , 58]. In our analysis of the eigenstates and energies of the radial component of the SQW QR Hamiltonian, $H_{\mathrm{SQW}, 0}^{(\tau)}$, we find that both the secular equation [Eqn. (4.12)] and eigenstates [Sec. (4.1.3)] agree with those of Refs. 7, 58]. However the ring-confinement of these systems have only been explored with a strong numerical focus due to their complexity [6]. Using perturbation theory and the approximation $\epsilon \quad$, we were able to derive a general subband dispersion [see Eqn. 4.48)]. Although this equation is not yet in an elegant format, compared to the dispersion equations of SLG and TMD, it is still a new result which allows for the analytical exploration of the parameter dependences (such as ring width $W$, band gap $\xi_{\mathrm{M}}$, etc.) of the band structure and transport properties in SQW QR.

We also explored the topological properties of these materials. For both SLG and SQW QRs, we found that as we moved deep into the inverted gap regime (i.e. band structure is strongly inverted), the lowest-subbands $(|n|=1)$ eventually became topologically non-trivial and hence possessed topologically protected edge states (TPES). For SLG, the limit at which this happens was found to be when $V_{0} /(\hbar v / W) \leq-1$, below which the subbands become topologically protected [see Eqn. (3.13)]. This limit turns out to greatly affect the Berry phase and thus using these results, experimentalists should be able to determine whether the QR is in the topological phase or not.

In the case of SQW QRs, the critical limits, at which the ring entered its topological phase, took much more complicated forms [see Sec. (4.1.2)] due to the lack of energy-reflection symmetry when the parameter $\tilde{D} \sigma 0$. This asymmetry meant that as we increased the inversion of the band gap, the $|n|=1$ bound states traversed into the gap at different gap values, respectively. This gave rise to two critical gap values, $\xi_{\mathrm{Mc} \pm}<0$, that are described by Eqn. (4.28) for a large width $\left(q_{0} W \gtrsim 10\right)$. In particular, when $\tilde{D}=0$, 
energy-reflection symmetry once again holds which leads to $\xi_{\mathrm{Mc}} q_{0} W \lesssim-1$ [Eqn. 4.29] ]. This limit is reminiscent to that of the SLG QR, thus exemplifying the Dirac-like nature of SQW QRs.

We can conclude from these findings that ring confinement tends to destroy the topological properties of subbands. Unconfined SLG and SQW systems usually possess TPESs for any inverted band gap value [2,70]. However once we confine them in a ring, TPESs only arise when the system parameters satisfy a critical limit (e.g. $V_{0} /(\hbar v / W) \leq-1$ for SLG QR and $\xi_{\text {Mc }} q_{0} W \lesssim-1$ for energy-reflection symmetric SQW QR). The underlying reason for this is that increasing the confinement (e.g. decreasing the width $W$ ) generally increases the magnitude of the confinement energies thus pushing TPES out of the gap. This phenomenon is analogous to the critical width that is present in the HgTe quantum well layer structure [20], as discussed in Sec. (1.5).

For SLG QRs, we found that the relative positions of the $\tau= \pm 1$ valley dispersions swapped for $|n|>1$ subbands as $V_{0}$ was tuned from topological $\left[V_{0} /(\hbar v / W) \leq-1\right]$ to the trivial $\left[V_{0} /(\hbar v / W)>-1\right]$ regions. In particular, a zero shift between the subband dispersions was present at $V_{0} /(\hbar v / W)=-1$. However for the $|n|=1$ subbands, this dispersion swapping did not take place which indicated a qualitative difference between the topological and non-topological subbands. In the SQW QR systems, we found similar results where the dispersions of higher subbands $(|n|>1)$ swapped positions while the lowest subbands $(|n|=1)$ did not.

In Chapters 5 and 6 we derived and analysed the analytical expressions of the Berry phase, $\theta_{\mathrm{g}}^{(\tau, n)}$, for SLG, TMD and SQW QRs. In previous work, this phase has been only analytically studied using non-material specific QR models $29,71,72]$. Our work, in this area, is consequential because we have discovered a correction term to the Berry phase, $\delta \theta_{\mathrm{g}}^{(n)}$, that arises purely from the ring confinement of our specific materials (SLG, TMD and SQW). Moreover, we also determined explicit expressions of $\theta_{\mathrm{g}}^{(\tau, n)}$.

In Chapter 5, we also found that the magnitude of the shift between $\tau$ flavour dispersions was equivalent (by a constant factor) to the magnitude of confinement arising Berry phase correction $\delta \theta_{\mathrm{g}}^{(n)}$. Furthermore, in Chapters 5 and 6 . these terms were discovered to be proportional to the diagonal $\mathbf{H}_{\alpha, 1}^{(\tau, n)}$ terms: $\Gamma_{\alpha}$ for SLG \& TMD, and $\Lambda_{\mathrm{SQW}}$ and $\Lambda_{\mathrm{SQW}}^{\prime}$ for SQW QRs, when 
the lead injection energy is approximately the size-quantisation energy, i.e. $E_{\text {in }} \approx E_{\alpha, 0}^{(\tau, n)}$. Additionally, $\delta \theta_{\mathrm{g}}^{(n)}$ and the shift between flavour dispersions were also found to be related to the $\phi$ separation of the $\tau= \pm 1$ flavour conductance peaks [see Sec. (5.1.1)]. Analogous to the swapping of the $|n|>1$ $\tau$ flavour dispersions, the $|n|>1$ conductance peaks also swapped positions at $\phi=\phi_{0}(m-1 / 2)$ when the gap size was varied [see Fig. 5.8b] ].

Generally, relativistic fermionic particles possess a spin $1 / 2$ Berry phase $\theta_{\mathrm{g}}^{(\tau, n)} \rightarrow \pi$ in the presence of a magnetic field. Particles that present this phase, we call "Dirac-like". This contrasts the non-relativistic Schrödinger equation which does not take into account the spin of a particle and thus does not predict the $\pi$ Berry phase. We call particles that behave spinless, as per the Schrödinger description, "Schrödinger-like". This description becomes important for distinguishing the lowest from the higher subbands, as was seen in Chapter 6 .

We saw that for both SLG and SQW QRs, the Berry phase of the lowest subband exhibited distinct qualitative differences compared to the higher subbands in the deeply inverted regime. As $\xi_{\mathrm{M}} q_{0} W \rightarrow \infty$, the lowest subband went as $\delta \theta_{\mathrm{g}}^{(|n|=1)} \rightarrow 0$ such that the total Berry phase became $\theta_{\mathrm{g}}^{(\tau, n)} \rightarrow$ $2 \pi \phi / \phi_{0}+\pi$ which meant the subband retained its Dirac spin $1 / 2, \pi$, phase. Additionally, the phase always stayed a positive value for $n=1$ and a negative value for $n=-1$. Furthermore, neither subband possessed a $\delta \theta_{\mathrm{g}}^{(|n|=1)}=0$ crossover point.

For SLG QRs, the higher subbands go as $\delta \theta_{\mathrm{g}}^{(|n|>1)} \rightarrow 1$ and thus $\theta_{\mathrm{g}}^{(\tau, n)} \rightarrow$ $2 \pi \phi / \phi_{0}$ which means it becomes more Schrödinger-like. Additionally, as we varied the gap size, $\delta \theta_{\mathrm{g}}^{(|n|>1)}$ of the higher subbands transitioned from positive to negative with a crossover point at $V_{0} /(\hbar v / W)=-1$ (i.e. when $\Gamma_{\mathrm{SLG}}=0$ ), as was indicated by the $\tau$ energy dispersion and conductance peak swapping. For SQW QRs, this swapping also occurred for the higher subbands. However, for $\xi_{\mathrm{M}} \rightarrow-\infty$, they did not seem to approach a welldefined limit due to the inclusion of the $\Upsilon_{\mathrm{SQW}}$ and $\Upsilon_{\mathrm{SQW}}^{\prime}$ terms. Thus for both SLG and SQW QRs, the higher subbands, which are always topologically trivial, behave significantly different from the lowest subbands, which become topologically protected, in the deeply inverted gap regime.

Overall, we have seen that $\delta \theta_{\mathrm{g}}^{(n)}$, that arises from the momentum depen- 
dent energy shift in the material dispersions, notably impacts the overall Berry phase, $\theta_{\mathrm{g}}^{(\tau, n)}$, the conductance and the dispersion relations. Hence, the momentum dependent energy shifts must be taken into account when considering transport properties of QR systems.

\section{Further outlook}

Although we have comprehensively understood the confinement effects and transport properties of SLG and TMD QR systems, we are still lacking the same thorough insight with regards to the SQW QR systems. In particular, the underlying behaviour of the Berry phase should be further investigated. Our understanding was partially hindered due to the complexity of the SQW subband dispersion equation which ideally could be simplified to be just in terms of the size-quantisation energy and system parameters, as was done for the SLG and TMD QRs.

We only analysed the conductance of a perfectly symmetric ring. However, the next steps would be to add asymmetry with regards to the relative positions of the junctions and include realistic impurity scatterers in the ring branches, as was suggested by Büttiker et. al. [35. This would add an extra layer of complexity that would however be rewarded with findings that are more likely to resemble the results obtained by experimentalists. It may also be useful to investigate the effects of a tilted magnetic-field that penetrates the ring.

For now, we hope that these results will aid experimentalists in understanding the complex QR conductance patterns that arise in SLG, TMD and SQW QR systems and support theorists in their attempt to understand transport phenomena in confined chiral charge carrier systems in further depth. 


\section{Appendix A}

\section{Extra information regarding SQW}

\section{A.1 Important signs of the SQW model}

The sign of $\sigma \equiv \operatorname{sgn}\left(\tilde{E}+\tilde{\kappa}^{2}+\xi_{\mathrm{M}}\right)$ and various other quantities are presented in Tables A.1 and A.2 for different ranges of $\xi_{\mathrm{M}}$ and energy values.

$\xi_{\mathrm{M}}$ region $\quad$ Energy region $\operatorname{sgn}\left(\tilde{E}+\tilde{\kappa}^{2}+\xi_{\mathrm{M}}\right) \cdot \operatorname{sgn}\left(\tilde{E}-\tilde{\lambda}^{2}+\xi_{\mathrm{M}}\right)$

\begin{tabular}{cccc}
\hline \hline & & \\
$\xi_{\mathrm{M}}>0$ & $\left|\xi_{\mathrm{M}}\right|>|\tilde{E}|$ & 1 & -1 \\
$\xi_{\mathrm{M}}<0$ & $\left|\xi_{\mathrm{M}}\right|>|\tilde{E}|$ & -1 & -1 \\
All & $\tilde{E}>\left|\xi_{\mathrm{M}}\right|$ & 1 & -1 \\
All & $\tilde{E}<\left|\xi_{\mathrm{M}}\right|$ & -1 & -1
\end{tabular}

Table A.1: Table of $\operatorname{sgn}\left(\tilde{E}+\tilde{\kappa}^{2}+\xi_{\mathrm{M}}\right)$ and $\operatorname{sgn}\left(\tilde{E}-\tilde{\lambda}^{2}+\xi_{\mathrm{M}}\right)$ for relevant regions of consideration.

\section{A.2 Comparing our formulation with B. Zhou's}

Here we show that the secular equation for a SQW QR is equivalent to the one presented by B. Zhou et. al. [7. Inverting the $H_{\mathrm{SQW}, 0}^{(\tau)}$ secular energy 
$\xi_{\mathrm{M}}$ region $\quad$ Energy region $\operatorname{sgn}\left(\tilde{E}-\tilde{\kappa}^{2}-\xi_{\mathrm{M}}\right) \quad \operatorname{sgn}\left(\tilde{E}+\tilde{\lambda}^{2}-\xi_{\mathrm{M}}\right)$

\begin{tabular}{cccc}
\hline \hline & & & \\
$\xi_{\mathrm{M}}>0$ & $\left|\xi_{\mathrm{M}}\right|>|\tilde{E}|$ & -1 & 1 \\
$\xi_{\mathrm{M}}<0$ & $\left|\xi_{\mathrm{M}}\right|>|\tilde{E}|$ & 1 & 1 \\
All & $\tilde{E}>\left|\xi_{\mathrm{M}}\right|$ & 1 & 1 \\
All & $\tilde{E}<\left|\xi_{\mathrm{M}}\right|$ & -1 & 1
\end{tabular}

Table A.2: Table of $\operatorname{sgn}\left(\tilde{E}-\tilde{\kappa}^{2}-\xi_{\mathrm{M}}\right)$ and $\operatorname{sgn}\left(\tilde{E}+\tilde{\lambda}^{2}-\xi_{\mathrm{M}}\right)$ for relevant regions of consideration.

equation [Eqn. (4.11)] gives us

$$
\frac{\cos (\tilde{\kappa} \tilde{W}) \cosh (\tilde{\lambda} \tilde{W})-1}{\sin (\tilde{\kappa} \tilde{W}) \sinh (\tilde{\lambda} \tilde{W})}=\operatorname{sgn}\left(\tilde{E}_{\kappa}+\xi_{M}+\tilde{\kappa}^{2}\right) \frac{\bar{\gamma}_{\lambda}^{2} \gamma_{\kappa}^{2}-1}{2 \bar{\gamma}_{\lambda} \gamma_{\kappa}}
$$

The LHS can be reworked to give

$$
\frac{\tanh \left(\frac{\tilde{\lambda} \tilde{W}}{2}\right)}{\tan \left(\frac{\tilde{\kappa} \tilde{W}}{2}\right)}-\frac{\tan \left(\frac{\tilde{\kappa} \tilde{W}}{2}\right)}{\tanh \left(\frac{\tilde{\lambda} \tilde{W}}{2}\right)}=\operatorname{sgn}\left(\tilde{E}_{\kappa}+\xi_{\mathrm{M}}+\tilde{\kappa}^{2}\right) \frac{\bar{\gamma}_{\lambda}^{2} \gamma_{\kappa}^{2}-1}{\bar{\gamma}_{\lambda} \gamma_{\kappa}}
$$

Now, taking a closer look at $\gamma_{\kappa}$ and $\bar{\gamma}_{\lambda}$, we see that

$$
\begin{gathered}
\gamma_{\kappa}=\sqrt{\frac{\tilde{E}_{\kappa}-\xi_{\mathrm{M}}-\tilde{\kappa}^{2}}{\tilde{E}_{\kappa}+\xi_{\mathrm{M}}+\tilde{\kappa}^{2}}}=\operatorname{sgn}\left(\tilde{E}_{\kappa}+\xi_{\mathrm{M}}+\tilde{\kappa}^{2}\right) \frac{\tilde{E}_{\kappa}-\xi_{\mathrm{M}}-\tilde{\kappa}^{2}}{\tilde{\kappa}} \\
=\operatorname{sgn}\left(\tilde{E}_{\kappa}+\xi_{\mathrm{M}}+\tilde{\kappa}^{2}\right) \frac{\tilde{\alpha}_{\tilde{\kappa}}}{\tilde{\kappa}} \\
\bar{\gamma}_{\lambda}=\sqrt{\frac{\tilde{\lambda}^{2}-\xi_{\mathrm{M}}-\tilde{E}_{\lambda}}{\tilde{\lambda}^{2}-\xi_{\mathrm{M}}+\tilde{E}_{\lambda}}}=\frac{\tilde{\lambda}}{\tilde{E}_{\lambda}-\xi_{\mathrm{M}}+\tilde{\lambda}^{2}}=\frac{\tilde{\lambda}}{\tilde{\alpha}_{\tilde{\lambda}}} .
\end{gathered}
$$

where $\tilde{\alpha}_{\tilde{\kappa}} \equiv \tilde{E}_{\kappa}-\xi_{\mathrm{M}}-\tilde{\kappa}^{2}$ and $\tilde{\alpha}_{\tilde{\lambda}} \equiv \tilde{E}_{\lambda}-\xi_{\mathrm{M}}+\tilde{\lambda}^{2}$. Substituting these expressions into the RHS in Equation A.2, we find

$$
\frac{\tanh \left(\frac{\tilde{\lambda} \tilde{W}}{2}\right)}{\tan \left(\frac{\tilde{\kappa} \tilde{W}}{2}\right)}-\frac{\tan \left(\frac{\tilde{\kappa} \tilde{W}}{2}\right)}{\tanh \left(\frac{\tilde{\lambda} \tilde{W}}{2}\right)}=\frac{\tilde{\alpha}_{\tilde{\kappa}}^{2} \tilde{\lambda}^{2}-\tilde{\alpha}_{\tilde{\lambda}}^{2} \tilde{\kappa}^{2}}{\tilde{\alpha}_{\tilde{\lambda}} \alpha_{\tilde{\kappa}} \tilde{\kappa} \tilde{\lambda}}
$$


which is identical to the secular equation by Zhou et. al. for which we can substitute $\tilde{\lambda} \rightarrow \tilde{\lambda}_{1}$ and $\tilde{\kappa} \rightarrow-i \tilde{\lambda}_{2}$.

\section{A.3 General SQW coefficients for wavefunc- tion}

$$
c_{1 \kappa}^{(\tau, n)} \rightarrow \frac{c_{2 \lambda}^{(\tau, n)} \bar{\gamma}_{\lambda} e^{\frac{1}{2} i \tilde{W}(\tilde{\kappa}+i \tilde{\lambda})}\left(-\sigma \gamma_{\kappa} \bar{\gamma}_{\lambda}+e^{2 \tilde{\lambda} \tilde{W}}\left(\sigma \gamma_{\kappa} \bar{\gamma}_{\lambda}+i\right)-2 i e^{\tilde{W}(\tilde{\lambda}+i \tilde{\kappa})}+i\right)}{\sigma \gamma_{\kappa} \bar{\gamma}_{\lambda}-2 \sigma \gamma_{\kappa} \bar{\gamma}_{\lambda} e^{\tilde{W}(\tilde{\lambda}+i \tilde{\kappa})}+e^{2 i \tilde{\kappa} \tilde{W}}\left(\sigma \gamma_{\kappa} \bar{\gamma}_{\lambda}+i\right)-i}
$$

$c_{2 \kappa}^{(\tau, n)} \rightarrow \frac{c_{2 \lambda}^{(\tau, n)} \bar{\gamma}_{\lambda} e^{\frac{1}{2} i \tilde{W}(\tilde{\kappa}+i \tilde{\lambda})}\left(e^{i \tilde{\kappa} \tilde{W}}\left(-\sigma \gamma_{\kappa} \bar{\gamma}_{\lambda}+e^{2 \tilde{\lambda} \tilde{W}}\left(\sigma \gamma_{\kappa} \bar{\gamma}_{\lambda}-i\right)-i\right)+2 i e^{\tilde{\lambda} \tilde{W}}\right)}{\sigma \gamma_{\kappa} \bar{\gamma}_{\lambda}-2 \sigma \gamma_{\kappa} \bar{\gamma}_{\lambda} e^{\tilde{W}(\tilde{\lambda}+i \tilde{\kappa})}+e^{2 i \tilde{\kappa} \tilde{W}}\left(\sigma \gamma_{\kappa} \bar{\gamma}_{\lambda}+i\right)-i}$

$$
c_{1 \lambda}^{(\tau, n)} \rightarrow-\frac{c_{2 \lambda}^{(\tau, n)}\left(-2 \sigma \gamma_{\kappa} \bar{\gamma}_{\lambda} e^{i \tilde{\kappa} \tilde{W}}+\left(\sigma \gamma_{\kappa} \bar{\gamma}_{\lambda}-i\right) e^{\tilde{W}(\tilde{\lambda}+2 i \tilde{\kappa})}+e^{\tilde{\lambda} \tilde{W}}\left(\sigma \gamma_{\kappa} \bar{\gamma}_{\lambda}+i\right)\right)}{\sigma \gamma_{\kappa} \bar{\gamma}_{\lambda}-2 \sigma \gamma_{\kappa} \bar{\gamma}_{\lambda} e^{\tilde{W}(\tilde{\lambda}+i \tilde{\kappa})}+e^{2 i \tilde{\kappa} \tilde{W}}\left(\sigma \gamma_{\kappa} \bar{\gamma}_{\lambda}+i\right)-i} .
$$

The wavefunction $\chi_{\mathrm{SQW}}^{(\tau, n)}(r)=\left(\chi_{\mathrm{SQW}, \uparrow}^{(\tau, n)}(r), \chi_{\mathrm{SQW}, \downarrow}^{(\tau, n)}(r)\right)^{T}$ is then given by

$$
\begin{aligned}
\chi_{\mathrm{SQW}, \uparrow}^{(\tau, n)}(\tilde{r})=-\tilde{c}_{2 \lambda}^{(\tau, n)} \sqrt{ } \bar{q}_{0} \bar{\gamma}_{\lambda}\left[\sin (\tilde{\kappa} \tilde{W}) \cosh \left(\tilde{\lambda}(\tilde{r}-\tilde{R})-\tilde{\lambda} \frac{\tilde{W}}{2}\right)\right. \\
+\cosh (\tilde{\lambda} \tilde{W}) \sin \left(\tilde{\kappa}(\tilde{r}-\tilde{R})-\tilde{\kappa} \frac{\tilde{W}}{2}\right)-\sin \left(\tilde{\kappa}(\tilde{r}-\tilde{R})+\tilde{\kappa} \frac{\tilde{W}}{2}\right) \\
-\sigma \gamma_{\kappa} \bar{\gamma}_{\lambda}\left(\cos (\tilde{\kappa} \tilde{W}) \sinh \left(\tilde{\lambda}(\tilde{r}-\tilde{R})-\tilde{\lambda} \frac{\tilde{W}}{2}\right)-\sinh \left(\tilde{\lambda}(\tilde{r}-\tilde{R})+\tilde{\lambda} \frac{\tilde{W}}{2}\right)\right. \\
\left.\left.+\sinh (\tilde{\lambda} \tilde{W}) \cos \left(\tilde{\kappa}(r-R)-\tilde{\kappa} \frac{\tilde{W}}{2}\right)\right)\right]
\end{aligned}
$$


and

$$
\begin{array}{r}
\chi_{\mathrm{SQW}, \downarrow}^{(\tau, n)}(\tilde{r})=i \tilde{c}_{2 \lambda}^{(\tau, n)} \sqrt{q_{0}} \tau\left[\sigma \gamma _ { \kappa } \overline { \gamma } _ { \lambda } \left(\cosh (\tilde{\lambda} \tilde{W}) \cos \left(\tilde{\kappa}(\tilde{r}-\tilde{R})-\tilde{\kappa} \frac{\tilde{W}}{2}\right)\right.\right. \\
-\cos \left(\tilde{\kappa}(\tilde{r}-\tilde{R})+\tilde{\kappa} \frac{\tilde{W}}{2}\right)+\cos (\tilde{\kappa} \tilde{W}) \cosh \left(\tilde{\lambda}(\tilde{r}-\tilde{R})-\tilde{\lambda} \frac{\tilde{W}}{2}\right) \\
\left.-\cosh \left(\tilde{\lambda}(\tilde{r}-\tilde{R})+\tilde{\lambda} \frac{\tilde{W}}{2}\right)\right)+\gamma_{\kappa}^{2} \bar{\gamma}_{\lambda}^{2} \sinh (\tilde{\lambda} \tilde{W}) \sin \left(\tilde{\kappa}(\tilde{r}-\tilde{R})-\tilde{\kappa} \frac{\tilde{W}}{2}\right) \\
\left.-\sin (\tilde{\kappa} \tilde{W}) \sinh \left(\tilde{\lambda}(\tilde{r}-\tilde{R})-\tilde{\lambda} \frac{\tilde{W}}{2}\right)\right] .
\end{array}
$$

Eqns. A.9 and (A.10) can be shown to be consistent with Eqns. 4.31) and 4.32 by substituting the relevant expression of $\tilde{E}$ [i.e. Eqns. 4.16 and (4.17)] into the $\gamma_{\kappa}$ and $\bar{\gamma}_{\lambda}$ of the more general wavefunction.

\section{A.4 $\tilde{E}<\left|\xi_{\mathrm{M}}\right|$ wavefunctions}

For $\tilde{E}<\left|\xi_{\mathrm{M}}\right|$ and $0>\xi_{\mathrm{M}}>-1 / 4$, we will have $\kappa \equiv i \bar{\kappa}$ and $\gamma_{\kappa} \equiv i \bar{\gamma}_{\bar{\kappa}}$, where

$$
\begin{aligned}
\tilde{\bar{\kappa}} & =q_{0}\left[\xi_{\mathrm{M}}-\frac{1}{2}\left(\sqrt{1+4 \xi_{\mathrm{M}}+4 \tilde{E}_{i \bar{\kappa}}^{2}}-1\right)\right]^{\frac{1}{2}}, \\
\bar{\gamma}_{\bar{\kappa}} & =\left[\frac{\frac{1}{2}\left(\sqrt{1+4 \xi_{\mathrm{M}}+4 \tilde{E}_{i \bar{\kappa}}^{2}}-1\right)-\tilde{E}_{i \bar{\kappa}}}{\frac{1}{2}\left(\sqrt{1+4 \xi_{\mathrm{M}}+4 \tilde{E}_{i \bar{\kappa}}^{2}}-1\right)+\tilde{E}_{i \bar{\kappa}}}\right]^{\frac{1}{2}}
\end{aligned}
$$

are then real numbers. For $\phi_{\mathrm{SQW}, 1,<}^{(\tau, n)}(\tilde{r})$, we have

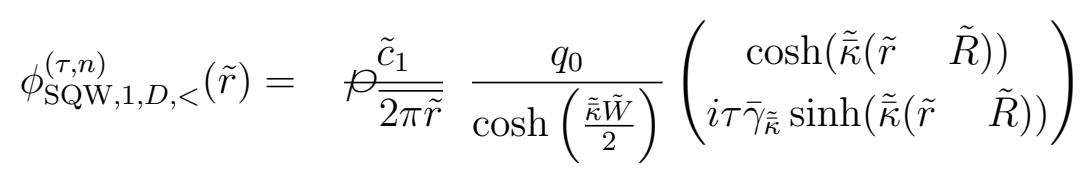


and

$$
\phi_{\mathrm{SQW}, 1, W}^{(\tau, n)}(\tilde{r})=\sqrt{ } \frac{\tilde{c}_{1}}{\overline{2 \pi \tilde{r}}} \frac{q_{0}}{\cosh \left(\frac{\tilde{\lambda} \tilde{W}}{2}\right)}\left(\begin{array}{c}
\cosh (\tilde{\lambda}(\tilde{r}-\tilde{R})) \\
i \tau \frac{1}{\bar{\gamma}_{\tilde{\lambda}}} \sinh (\tilde{\lambda}(\tilde{r}-\tilde{R}))
\end{array}\right)
$$

and for $\phi_{\mathrm{SQW}, 2,<}^{(\tau, n)}(r)$, we have

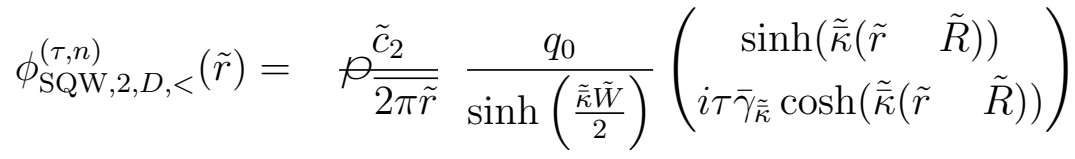

and

$$
\phi_{\mathrm{SQW}, 2, W}^{(\tau, n)}(\tilde{r})=\sqrt{\frac{\tilde{c}_{2}}{\overline{2 \pi \tilde{r}}}} \frac{q_{0}}{\sinh \left(\frac{\tilde{\lambda} \tilde{W}}{2}\right)}\left(\begin{array}{c}
\sinh (\tilde{\lambda}(\tilde{r}-\tilde{R})) \\
i \tau \frac{1}{\overline{\gamma_{\tilde{\lambda}}}} \cosh (\tilde{\lambda}(\tilde{r}-\tilde{R}))
\end{array}\right)
$$

where we have noted that $\sigma=-1$ for the regime of interest. 


\section{Appendix B}

\section{Useful integrals}

Here we present the integrals that are required to solve for the $\mathbf{H}_{\alpha, 1}^{(\tau)}$ matrix coefficients. We have Taylor-expanded the solutions up to the second order of $\epsilon$.

$$
\begin{aligned}
& \int_{-\frac{1}{2}}^{\frac{1}{2}} \frac{\epsilon}{(1+\epsilon \eta)} \cos \left(\lambda_{\alpha} \eta W\right) \cos \left(\lambda_{\beta} \eta W\right) d \eta=\frac{\epsilon}{\tilde{W}}\left[\frac{\sin \left(\frac{\left(\lambda_{\alpha}-\lambda_{\beta}\right) W}{2}\right)}{\lambda_{\alpha}-\lambda_{\beta}}+\frac{\sin \left(\frac{\left(\lambda_{\alpha}+\lambda_{\beta}\right) W}{2}\right)}{\lambda_{\alpha}+\lambda_{\beta}}\right](\text { B.1) } \\
&+ O\left(\epsilon^{3}\right) \\
& \int_{-\frac{1}{2}}^{\frac{1}{2}} \frac{\epsilon}{(1+\epsilon \eta)} \sin \left(\lambda_{\alpha} \eta W\right) \sin \left(\lambda_{\beta} \eta W\right) d \eta=\frac{\epsilon}{\tilde{W}}\left[\frac{\sin \left(\frac{\left(\lambda_{\alpha}-\lambda_{\beta}\right) W}{2}\right)}{\lambda_{\alpha}-\lambda_{\beta}}-\frac{\sin \left(\frac{\left(\lambda_{\alpha}+\lambda_{\beta}\right) W}{2}\right)}{\lambda_{\alpha}+\lambda_{\beta}}\right] \\
&+O\left(\epsilon^{3}\right)
\end{aligned}
$$




$$
\begin{aligned}
& \int_{-\frac{1}{2}}^{\frac{1}{2}} \frac{\epsilon}{(1+\epsilon \eta)} \cos \left(\lambda_{\alpha} \eta W\right) \sin \left(\lambda_{\beta} \eta W\right) d \eta= \\
& \frac{\epsilon^{2}}{W^{2}}\left[-\frac{W}{2} \frac{\cos \left(\frac{\left(\lambda_{\alpha}-\lambda_{\beta}\right) W}{2}\right)}{\lambda_{\alpha}-\lambda_{\beta}}+\frac{W}{2} \frac{\cos \left(\frac{\left(\lambda_{\alpha}+\lambda_{\beta}\right) W}{2}\right)}{\lambda_{\alpha}+\lambda_{\beta}}\right. \\
& \left.+\frac{\sin \left(\frac{\left(\lambda_{\alpha}-\lambda_{\beta}\right) W}{2}\right)}{\left(\lambda_{\alpha}-\lambda_{\beta}\right)^{2}}-\frac{\sin \left(\frac{\left(\lambda_{\alpha}+\lambda_{\beta}\right) W}{2}\right)}{\left(\lambda_{\alpha}+\lambda_{\beta}\right)^{2}}\right]+O\left(\epsilon^{4}\right)
\end{aligned}
$$

$$
\begin{aligned}
\int_{-\frac{1}{2}}^{\frac{1}{2}} \frac{\epsilon}{(1+\epsilon \eta)} \cos \left(\lambda_{\beta} \eta W\right) \sin \left(\lambda_{\alpha} \eta W\right) d \eta= \\
\frac{\epsilon^{2}}{W^{2}}\left[\frac{W}{2} \frac{\cos \left(\frac{\left(\lambda_{\alpha}-\lambda_{\beta}\right) W}{2}\right)}{\lambda_{\alpha}-\lambda_{\beta}}+\frac{W}{2} \frac{\cos \left(\frac{\left(\lambda_{\alpha}+\lambda_{\beta}\right) W}{2}\right)}{\lambda_{\alpha}+\lambda_{\beta}}\right. \\
\left.-\frac{\sin \left(\frac{\left(\lambda_{\alpha}-\lambda_{\beta}\right) W}{2}\right)}{\left(\lambda_{\alpha}-\lambda_{\beta}\right)^{2}}-\frac{\sin \left(\frac{\left(\lambda_{\alpha}+\lambda_{\beta}\right) W}{2}\right)}{\left(\lambda_{\alpha}+\lambda_{\beta}\right)^{2}}\right]+O\left(\epsilon^{4}\right)
\end{aligned}
$$

$$
\begin{aligned}
& \int_{-\frac{1}{2}}^{\frac{1}{2}} \frac{\epsilon^{2}}{W(1+\epsilon \eta)^{2}} \cos \left(\lambda_{\alpha} \eta W\right) \cos \left(\lambda_{\beta} \eta W\right) d \eta= \\
& \frac{\epsilon^{2}}{\tilde{W}^{2}}\left[\frac{\sin \left(\frac{\left(\lambda_{\alpha}-\lambda_{\beta}\right) W}{2}\right)}{\lambda_{\alpha}-\lambda_{\beta}}+\frac{\sin \left(\frac{\left(\lambda_{\alpha}+\lambda_{\beta}\right) W}{2}\right)}{\lambda_{\alpha}+\lambda_{\beta}}\right]+O\left(\epsilon^{4}\right)
\end{aligned}
$$

$$
\begin{aligned}
\int_{-\frac{1}{2}}^{\frac{1}{2}} \frac{\epsilon^{2}}{W(1+\epsilon \eta)^{2}} \sin \left(\lambda_{\alpha} \eta W\right) \sin \left(\lambda_{\beta} \eta W\right) d \eta= \\
\frac{\epsilon^{2}}{\tilde{W}^{2}}\left[\frac{\sin \left(\frac{\left(\lambda_{\alpha}-\lambda_{\beta}\right) W}{2}\right)}{\lambda_{\alpha}-\lambda_{\beta}}-\frac{\sin \left(\frac{\left(\lambda_{\alpha}+\lambda_{\beta}\right) W}{2}\right)}{\lambda_{\alpha}+\lambda_{\beta}}\right]+O\left(\epsilon^{4}\right)
\end{aligned}
$$




$$
\begin{aligned}
& \int_{-\frac{1}{2}}^{\frac{1}{2}} \frac{\epsilon^{2}}{W(1+\epsilon \eta)^{2}} \cos \left(\lambda_{\alpha} \eta W\right) \sin \left(\lambda_{\beta} \eta W\right) d \eta=O\left(\epsilon^{3}\right) \\
& \int_{-\frac{1}{2}}^{\frac{1}{2}} \frac{\epsilon^{2}}{W(1+\epsilon \eta)^{2}} \cos \left(\lambda_{\beta} \eta W\right) \sin \left(\lambda_{\alpha} \eta W\right) d \eta=O\left(\epsilon^{3}\right)
\end{aligned}
$$




\section{Bibliography}

[1] Weiss, N. O. et al. Graphene: An emerging electronic material. Advanced Materials 24, 5782-5825 (2012). URL http://dx.doi.org/10.1002/ adma.201201482.

[2] Qi, X.-L. \& Zhang, S.-C. Topological insulators and superconductors. Rev. Mod. Phys. 83, 1057-1110 (2011).

[3] Xu, S.-Y. et al. Discovery of a weyl fermion semimetal and topological fermi arcs. Science 349, 613-617 (2015). URL http://science. sciencemag.org/content/349/6248/613.

[4] Viefers, S., Koskinenb, P., Deoc, P. S. \& Manninenb, M. Quantum rings for beginners: energy spectra and persistent currents. Physica E 21, 1-35 (2004).

[5] Recher, P. et al. Aharonov-Bohm effect and broken valley degeneracy in graphene rings. Phys. Rev. B 76, 235404 (2007).

[6] Michetti, P. \& Recher, P. Bound states and persistent currents in topological insulator rings. Phys. Rev. B 83, 125420 (2011).

[7] Zhou, B., Lu, H.-Z., Chu, R.-L., Shen, S.-Q. \& Niu, Q. Finite size effects on helical edge states in a quantum spin-hall system. Phys. Rev. Lett. 101, 246807 (2008).

[8] Oliveira, D., Fu, J., Villegas-Lelovsky, L., Dias, A. C. \& Qu, F. Valley Zeeman energy in monolayer $\mathrm{MoS}_{2}$ quantum rings: Aharonov-Bohm effect. Physical Review B 93, 205422- (2016). URL https://link. aps.org/doi/10.1103/PhysRevB.93.205422. 
[9] Russo, S. et al. Observation of Aharonov-Bohm conductance oscillations in a graphene ring. Phys. Rev. B 77, 085413 (2008).

[10] Yoo, J. S., Park, Y. W., Skákalová, V. \& Roth, S. Shubnikov-de Haas and Aharonov Bohm effects in a graphene nanoring structure. Applied Physics Letters 96, 143112 (2010). URL http://dx.doi.org/10.1063/ 1.3380616 .

[11] Huefner, M. et al. Investigation of the Aharonov-Bohm effect in a gated graphene ring. Phys. Status Solidi $B$ 246, 27562759 (2009). URL https://www.nano.phys.ethz.ch/ ihn/papers/ HuefnerPhysStatSol.pdf.

[12] Smirnov, D., Schmidt, H. \& Haug, R. J. Aharonov-Bohm effect in an electron-hole graphene ring system. Applied Physics Letters 100, 203114 (2012). URL http://dx.doi.org/10.1063/1.4717622.

[13] Fan, C. et al. Novel micro-rings of molybdenum disulfide $\left(\mathrm{MoS}_{2}\right)$. Nanoscale 6, 14652-14656 (2014). URL http://dx.doi.org/10.1039/ C4NR05111D.

[14] Samal, M., Barange, N., Ko, D.-H. \& Yun, K. Graphene quantum rings doped PEDOT:PSS based composite layer for efficient performance of optoelectronic devices. The Journal of Physical Chemistry C 119, 19619-19627 (2015). URL http://dx.doi.org/10.1021/acs. jpcc.5b05225.

[15] Gusynin, V. P. \& Sharapov, S. G. Unconventional integer quantum hall effect in graphene. Physical Review Letters 95, 146801- (2005). URL https://link.aps.org/doi/10.1103/PhysRevLett.95.146801.

[16] Novoselov, K. S. et al. Two-dimensional gas of massless Dirac fermions in graphene. Nature 438, 197-200 (2005). URL http://dx.doi.org/ $10.1038 /$ nature04233

[17] Geim, A. K. \& Novoselov, K. S. The rise of graphene. Nat Mater 6, 183-191 (2007). URL http://dx.doi.org/10.1038/nmat1849. 
[18] Castro Neto, A. H., Guinea, F., Peres, N. M. R., Novoselov, K. S. \& Geim, A. K. The electronic properties of graphene. Reviews of Modern Physics 81, 109-162 (2009). URL https://link.aps.org/doi/ 10.1103/RevModPhys.81.109.

[19] Kormányos, A. et al. $k \cdot p$ theory for two-dimensional transition metal dichalcogenide semiconductors. 2D Materials 2 (2015).

[20] Bernevig, B. A., Hughes, T. L. \& Zhang, S. Quantum spin Hall effect and topological phase transition in HgTe quantum wells. Science $\mathbf{3 1 4}$, 1757-1761 (2006).

[21] Lapushkin, I., Zakharova, A., Yen, S. T. \& Chao, K. A. A selfconsistent investigation of the semimetal-semiconductor transition in InAs/GaSb quantum wells under external electric fields. Journal of Physics: Condensed Matter 16, 4677 (2004). URL http://stacks. iop. org $/ 0953-8984 / 16 / i=26 / a=003$.

[22] Zhang, Y. et al. Direct observation of the transition from indirect to direct bandgap in atomically thin epitaxial $\mathrm{MoSe}_{2}$. Nat Nano 9, 111-115 (2014). URL http://dx.doi.org/10.1038/nnano.2013.277.

[23] Fomin, V. M. Physics of Quantum Rings, vol. 1 of NanoScience and Technology (Springer-Verlag Berlin Heidelberg, 2014).

[24] Aharonov, Y. \& Bohm, D. Significance of electromagnetic potentials in the quantum theory. Physical Review 115, 485-491 (1959). URL https://link.aps.org/doi/10.1103/PhysRev.115.485.

[25] Griffiths, D. Introduction to Quantum Mechanics (Pearson, 2013). URL https://books.google.co.nz/books?id=6xkLngEACAAJ

[26] Sakurai, J. \& Napolitano, J. Modern Quantum Mechanics (AddisonWesley, 2011). URL https://books.google.co.nz/books?id= N4I-AQAACAAJ.

[27] Berry, M. V. Quantal phase factors accompanying adiabatic changes. Proceedings of the Royal Society of London A: Mathematical, Physical and Engineering Sciences 392, 45-57 (1984). URL http://rspa. 
royalsocietypublishing. org/content/392/1802/45. http://rspa. royalsocietypublishing.org/content/392/1802/45.full.pdf.

[28] Zhang, Y., Tan, Y.-W., Stormer, H. L. \& Kim, P. Experimental observation of the quantum Hall effect and Berry's phase in graphene. Nature 438, 201-204 (2005). URL http://dx.doi.org/10.1038/ nature 04235 .

[29] Loss, D., Goldbart, P. \& Balatsky, A. V. Berry's phase and persistent charge and spin currents in textured mesoscopic rings. Physical Review Letters 65, 1655-1658 (1990). URL https://link.aps.org/doi/10. 1103/PhysRevLett.65.1655.

[30] Földi, P., Kálmán, O. \& Peeters, F. M. Stability of spintronic devices based on quantum ring networks. Physical Review B 80, 125324- (2009). URL https://link.aps.org/doi/10.1103/PhysRevB.80.125324.

[31] Popp, M., Frustaglia, D. \& Richter, K. Spin filter effects in mesoscopic ring structures. Nanotechnology 14, 347 (2003). URL http://stacks. iop.org/0957-4484/14/i=2/a=347.

[32] Radisavljevic, B., Radenovic, A., Brivio, J., Giacometti, V. \& Kis, A. Single-layer $\mathrm{MoS}_{2}$ transistors. Nat Nano 6, 147-150 (2011). URL http: //dx.doi.org/10.1038/nnano.2010.279.

[33] Wang, H. et al. Integrated circuits based on bilayer $\mathrm{MoS}_{2}$ transistors. Nano Letters 12, 4674-4680 (2012). URL http://dx.doi.org/10. $1021 / \mathrm{n} 1302015 \mathrm{v}$.

[34] König, M., Buhmann, H., Becker, C. R. \& Molenkamp, L. W. Phase effects in HgTe quantum structures. physica status solidi (c) 4, 33743381 (2007).

[35] Büttiker, M., Imry, Y. \& Azbel, M. Y. Quantum oscillations in onedimensional normal-metal rings. Phys. Rev. A 30, 1982-1989 (1984).

[36] Gefen, Y., Imry, Y. \& Azbel, M. Y. Quantum oscillations and the Aharonov-Bohm effect for parallel resistors. Phys. Rev. Lett. 52, 129 132 (1984). 
[37] Shapiro, B. Quantum conduction on a Cayley tree. Phys. Rev. Lett. 50, 747-750 (1983).

[38] Guhr, T., Müller-Groeling, A. \& Weidenmüller, H. A. Randommatrix theories in quantum physics: common concepts. Physics Reports 299, 189-425 (1998). URL http://www. sciencedirect.com/science/ article/pii/S0370157397000884

[39] Chen, X., Liu, Z.-X. \& Wen, X.-G. Two-dimensional symmetryprotected topological orders and their protected gapless edge excitations. Physical Review B 84, 235141- (2011). URL https://link.aps.org/ doi/10.1103/PhysRevB.84.235141.

[40] $\mathrm{Xu}, \mathrm{Y}$. et al. Observation of topological surface state quantum hall effect in an intrinsic three-dimensional topological insulator. Nat Phys 10, 956-963 (2014). URL http://dx.doi.org/10.1038/nphys3140.

[41] Nayak, C., Simon, S. H., Stern, A., Freedman, M. \& Das Sarma, S. Non-abelian anyons and topological quantum computation. Reviews of Modern Physics 80, 1083-1159 (2008). URL https://link.aps.org/ doi/10.1103/RevModPhys.80.1083.

[42] Maciejko, J., Kim, E.-A. \& Qi, X.-L. Spin Aharonov-Bohm effect and topological spin transistor. Physical Review B 82, 195409-(2010). URL https://link.aps.org/doi/10.1103/PhysRevB.82.195409.

[43] Wu, C., Bernevig, B. A. \& Zhang, S.-C. Helical liquid and the edge of quantum spin Hall systems. Physical Review Letters 96, 106401-(2006). URL https://link.aps .org/doi/10.1103/PhysRevLett.96.106401.

[44] Kane, C. L. \& Mele, E. J. Z 2 topological order and the quantum spin Hall effect. Physical Review Letters 95, 146802- (2005). URL https: //link.aps.org/doi/10.1103/PhysRevLett.95.146802.

[45] YU, P. \& Cardona, M. Fundamentals of Semiconductors: Physics and Materials Properties. v. 3 Berlin Heidelberg, 2005). URL https:// books.google.co.nz/books?id=W9pdJZoAeyEC.

[46] Kittel, C. Quantum theory of solids (Wiley, 1963). URL https:// books.google.co.nz/books?id=zgBRAAAMAAJ. 
[47] Jackiw, R. \& Templeton, S. How super-renormalizable interactions cure their infrared divergences. Phys. Rev. D 23, 2291-2304 (1981).

[48] Berry, M. V. \& Mondragon, R. J. Neutrino billiards: Time-reversal symmetry-breaking without magnetic fields. Proc. R. Soc. Lond. A $\mathbf{4 1 2}$, 53-74 (1987).

[49] Xiao, D., Liu, G.-B., Feng, W., Xu, X. \& Yao, W. Coupled spin and valley physics in monolayers of $\mathrm{MoS}_{2}$ and other group-VI dichalcogenides. Physical Review Letters 108, 196802- (2012). URL https: //link.aps.org/doi/10.1103/PhysRevLett.108.196802.

[50] Kormányos, A., Zólyomi, V., Drummond, N. D. \& Burkard, G. Erratum: Spin-orbit coupling, quantum dots, and qubits in monolayer transition metal dichalcogenides [phys. rev. x 4, 011034 (2014)]. Physical Review X 4, 039901-(2014). URL https://link.aps.org/doi/ 10.1103/PhysRevX.4.039901.

[51] Kormányos, A. et al. Monolayer $\mathrm{MoS}_{2}$ : Trigonal warping, the $\boldsymbol{\Gamma}$ valley, and spin-orbit coupling effects. Physical Review B 88, 045416- (2013). URL https://link.aps .org/doi/10.1103/PhysRevB .88.045416.

[52] König, M. et al. The quantum spin hall effect: Theory and experiment. J. Phys. Soc. Jpn. 77, 031007 (2008).

[53] Bromley, D. \& Greiner, W. Relativistic Quantum Mechanics. Wave Equations (Springer Berlin Heidelberg, 2013). URL https://books. google.co.nz/books?id=YCb0CAAAQBAJ.

[54] Winkler, R. \& Zülicke, U. Discrete symmetries of low-dimensional Dirac models: A selective review with a focus on condensed-matter realizations. ANZIAM J. 57, 3-17 (2015).

[55] Broido, D. A. \& Sham, L. J. Effective masses of holes at GaAs-AlGaAs heterojunctions. Phys. Rev. B 31, 888-892 (1985).

[56] Eric Yang, S.-R., Broido, D. A. \& Sham, L. J. Holes at GaAs-AlxGa1xAs heterojunctions in magnetic fields. Phys. Rev. B 32, 6630-6633 (1985). 
[57] Datta, S. Electron Transport in Mesoscopic Systems (Cambridge University Press, Cambridge, UK, 1995).

[58] White, S. R. \& Sham, L. J. Electronic properties of flat-band semiconductor heterostructures. Phys. Rev. Lett. 47, 879-882 (1981).

[59] McKellar, B. H. J. \& Stephenson, G. J. Relativistic quarks in onedimensional periodic structures. Phys. Rev. C 35, 2262-2271 (1987).

[60] Coutinho, F. A. B., Nogami, Y. \& Toyama, F. M. General aspects of the bound-state solutions of the one-dimensional Dirac equation. Am. J. Phys. 56, 904-907 (1988).

[61] Alberto, P., Fiolhais, C. \& Gil, V. M. S. Relativistic particle in a box. Eur. J. Phys. 17, 19 (1996).

[62] Hasegawa, H. Bound states of the one-dimensional Dirac equation for scalar and vector double square-well potentials. Physica E 59, $192-$ 201 (2014).

[63] Chodos, A., Jaffe, R. L., Johnson, K., Thorn, C. B. \& Weisskopf, V. F. New extended model of hadrons. Phys. Rev. D 9, 3471-3495 (1974).

[64] Chodos, A., Jaffe, R. L., Johnson, K. \& Thorn, C. B. Baryon structure in the bag theory. Phys. Rev. D 10, 2599-2604 (1974).

[65] Alonso, V. \& Vincenzo, S. D. General boundary conditions for a Dirac particle in a box and their non-relativistic limits. J. Phys. A: Math. Gen. 30, 8573 (1997).

[66] Zarenia, M., Pereira, J. M., Chaves, A., Peeters, F. M. \& Farias, G. A. Simplified model for the energy levels of quantum rings in single layer and bilayer graphene. Phys. Rev. B 81, 045431 (2010). Erratum: ibid. 82, 119906(E) (2010).

[67] Sticlet, D., Dóra, B. \& Cayssol, J. Persistent currents in Dirac fermion rings. Phys. Rev. B 88, 205401 (2013). URL http://link.aps.org/ doi/10.1103/PhysRevB.88.205401. 
[68] Bolívar, N., Medina, E. \& Berche, B. Persistent charge and spin currents in the long-wavelength regime for graphene rings. Phys. Rev. B 89, 125413 (2014).

[69] Ross, J. S. et al. Electrical control of neutral and charged excitons in a monolayer semiconductor. Nature Communications 4, 1474 EP - (2013). URL http://dx.doi .org/10.1038/ncomms2498.

[70] Pankratov, O. A., Pakhomov, S. V. \& Volkov, B. A. Supersymmetry in heterojunctions: Band-inverting contact on the basis of $\mathrm{Pb}_{1 \mathrm{x}} \mathrm{Sn}_{\mathrm{x}} \mathrm{Te}$ and $\mathrm{Hg}_{1 \mathrm{x}} \mathrm{Cd}_{\mathrm{x}} \mathrm{Te}$. Solid State Communications 61, 9396 (1987). URL http://www.sciencedirect.com/science/article/ pii/0038109887909343.

[71] Lopes-Oliveira, V., Castelano, L. K., Marques, G. E., Ulloa, S. E. \& Lopez-Richard, V. Berry phase and Rashba fields in quantum rings in tilted magnetic field. Physical Review B 92 (2015). URL http: //par.nsf.gov/biblio/10007541.

[72] Nagasawa, F., Frustaglia, D., Saarikoski, H., Richter, K. \& Nitta, J. Control of the spin geometric phase in semiconductor quantum rings. Nature Communications 4, 2526 EP - (2013). URL http://dx.doi. org/10.1038/ncomms3526. 